US Army Corps

of Engineers ${ }_{\circledast}$

Engineer Research and

Development Center

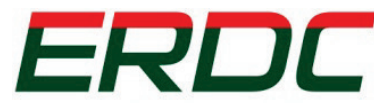

INNOVATIVE SOLUTIONS for a safer, better world

Coastal Inlets Research Program

\title{
Numerical Modeling of Wave Overtopping of Buffalo Harbor Confined Disposal Facility (CDF4)
}

Zeki Demirbilek, Lihwa Lin, Okey G. Nwogu, Michael C. Mohr,

Shanon Chader, and Geoffrey K. Hintz

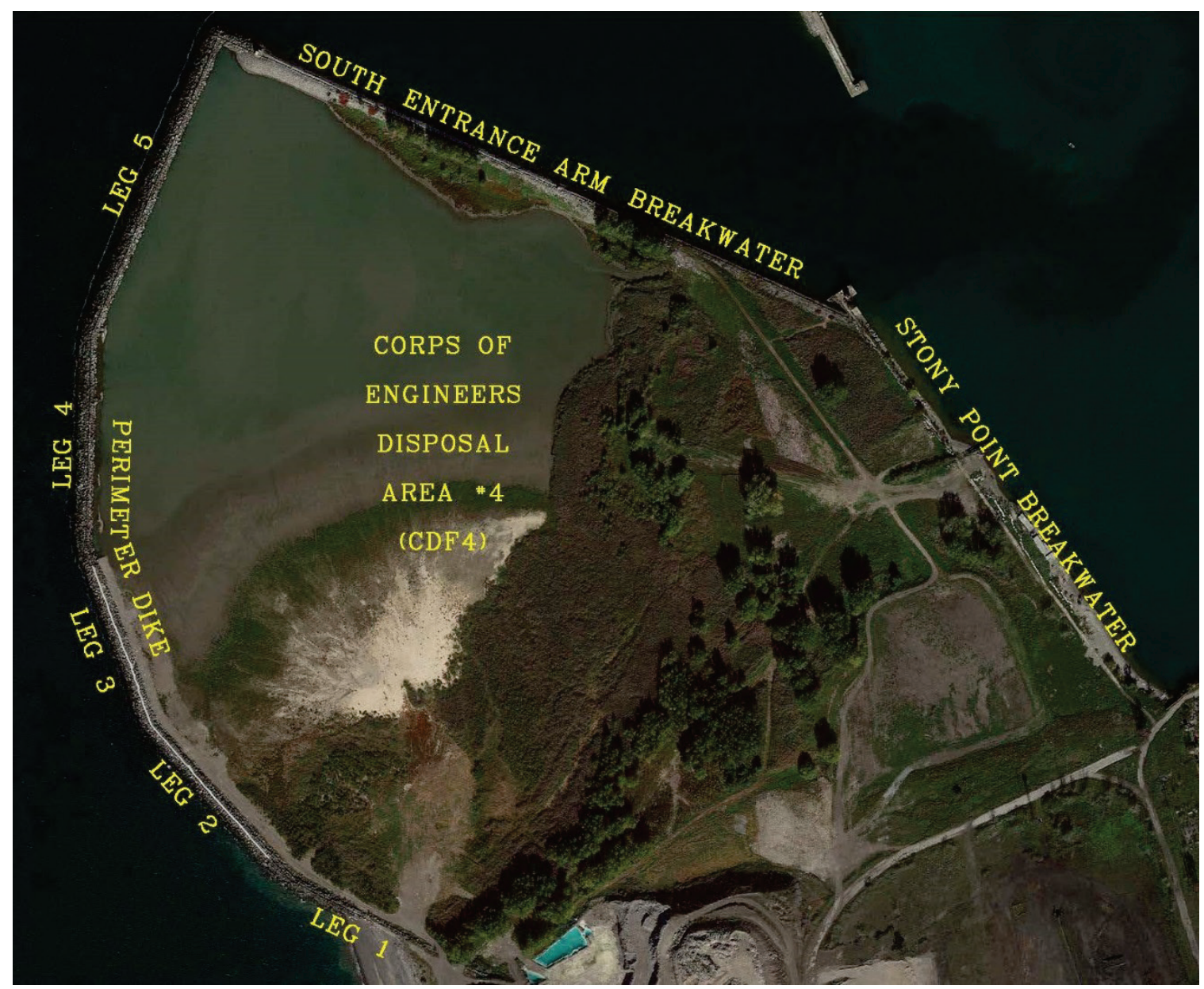


The U.S. Army Engineer Research and Development Center (ERDC) solves the nation's toughest engineering and environmental challenges. ERDC develops innovative solutions in civil and military engineering, geospatial sciences, water resources, and environmental sciences for the Army, the Department of Defense, civilian agencies, and our nation's public good. Find out more at www.erdc.usace.army.mil.

To search for other technical reports published by ERDC, visit the ERDC online library at http://acwc.sdp.sirsi.net/client/default. 


\section{Numerical Modeling of Wave Overtopping of Buffalo Harbor Confined Disposal Facility (CDF4)}

Zeki Demirbilek and Lihwa Lin

Coastal and Hydraulics Laboratory

U.S. Army Engineer Research and Development Center

3909 Halls Ferry Road

Vicksburg, MS 39180-6199

Okey G. Nwogu

University of Michigan

Department of Naval Architecture and Marine Engineering

2600 Draper Road

Ann Arbor, MI 48109-2145

Michael C. Mohr, Shanon Chader, and Geoffrey K. Hintz

U.S. Army Corps of Engineers, Buffalo District

1776 Niagara Street

Buffalo, NY 14207

Final report

Approved for public release; distribution is unlimited.

Prepared for U.S. Army Corps of Engineers, Buffalo District

1776 Niagara Street, Buffalo, NY 14207

Under Wave Structures Work Unit 280H46 


\section{Abstract}

This report provides details of a numerical modeling study conducted to provide estimates of wave overtopping of the Buffalo Harbor Confined Disposal Facility \#4 (CDF4). The CDF4 is situated adjacent to the South Entrance Channel to Buffalo Harbor, New York, along the south shoreline of the Lake Erie. This contaminated material holding facility experiences periodic overtopping during significant storm events. There is concern that overtopping waves may cause the contaminated fine-grain sediments inside the Confined Disposal Facility (CDF) to mobilize, some of which may get transported over the South Entrance Arm Breakwater (SEAB) into the south harbor entrance, navigation channel, and harbor complex. The present study focused on predictive estimates of waves and wave overtopping. Because flow and sediment transport modeling were not considered, it was assumed that if flow occurred over the SEAB, some sediment would exit also. Wave processes in the exterior and interior areas of $\mathrm{CDF} 4$ were investigated to determine wave runup and overtopping for the existing CDF system using two classes of wave models: CMS-Wave, a spectral wind-wave generation, growth and transformation model, and a nonlinear Boussinesq-type wave model (BOUSS-1D/2D). This report documents details of the modeling study, including data used in the study, results, and recommendations.

DISCLAIMER: The contents of this report are not to be used for advertising, publication, or promotional purposes. Citation of trade names does not constitute an official endorsement or approval of the use of such commercial products. All product names and trademarks cited are the property of their respective owners. The findings of this report are not to be construed as an official Department of the Army position unless so designated by other authorized documents. 


\section{Contents}
Abstract ii
Figures and Tables.
-...v

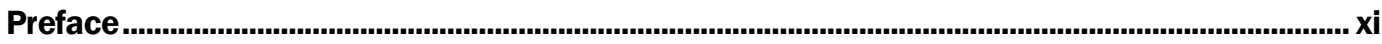
Unit Conversion Factors ............................................................................................................. xii
1 Introduction .................................................................................................................................. 1

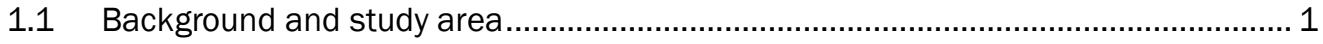
1.2 Condition of existing Confined Disposal Facility (CDF) ...................................... 3

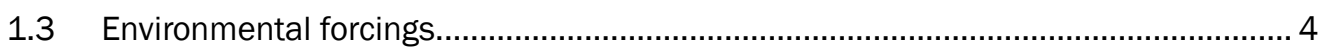

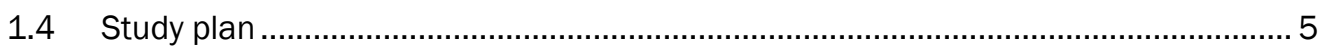

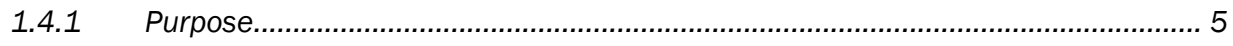

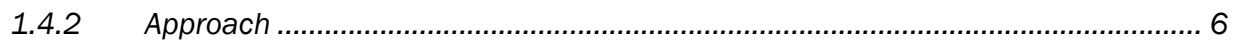

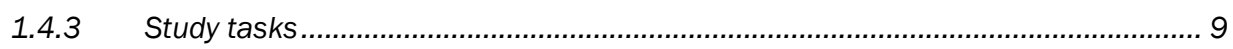

2 Data for the Study ................................................................................................................11

2.1 Bathymetry and coastline data.................................................................. 11

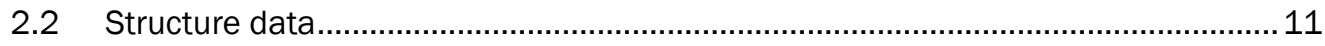

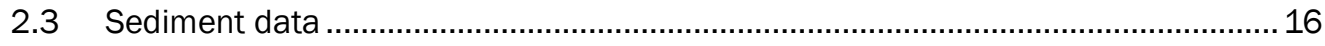

2.4 Maintenance dredging data ............................................................................ 17

2.5 Future Fill scenarios ........................................................................................... 18

2.6 Water level and river discharge data .................................................................. 18

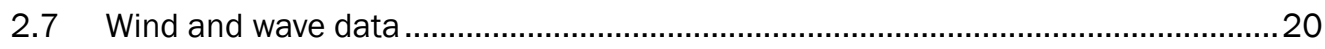

2.8 Storm data for modeling .................................................................................. 21

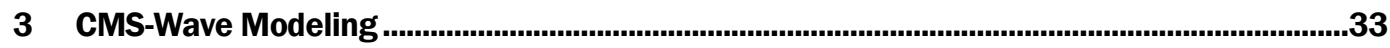

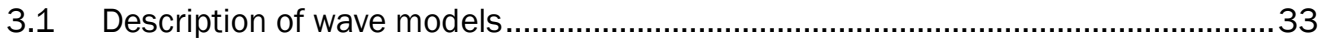

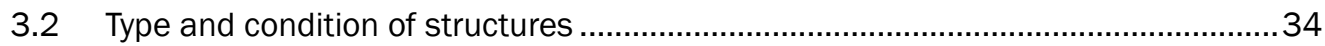

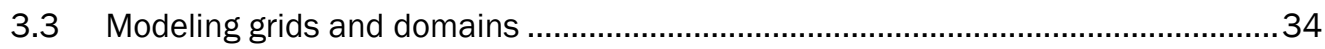

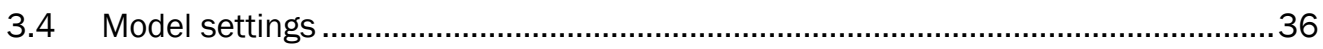

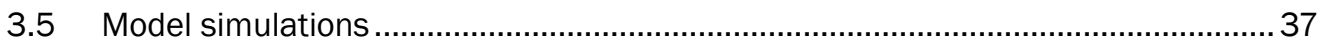

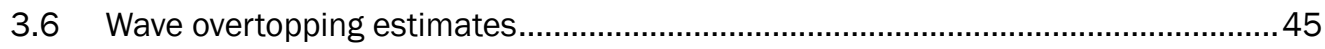

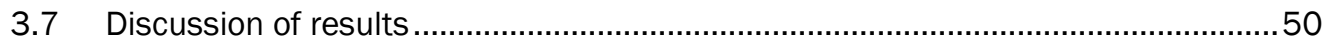

4 Boussinesq Wave Modeling..............................................................................................53

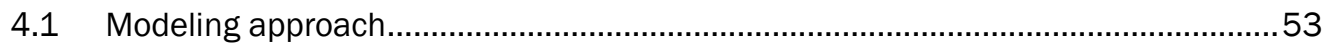

4.2 Model grids and configurations investigated ...................................................55

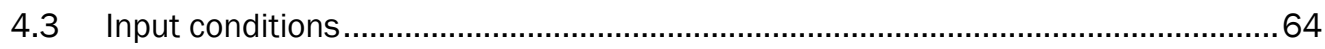

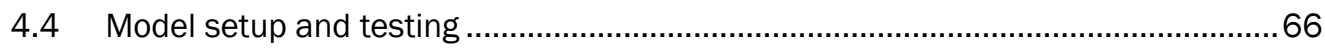

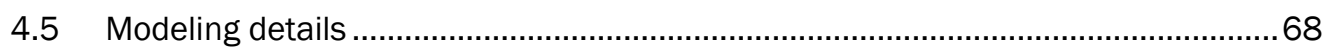

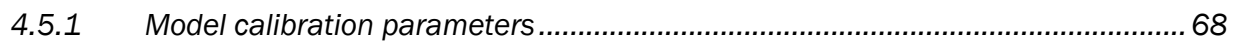

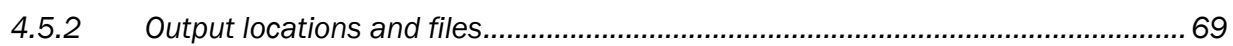

4.5.3 Results for storm conditions ........................................................................ 70 


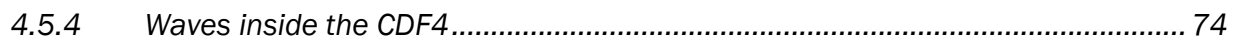

4.5.5 Images of instantaneous wave overtopping......................................................... 76

4.5.6 Results for selected storm conditions ............................................................... 84

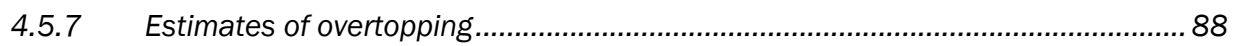

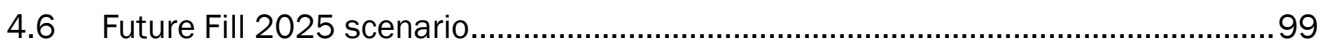

4.7 Future Fill 2036 scenario............................................................................ 104

4.8 Raised dike scenario ............................................................................ 108

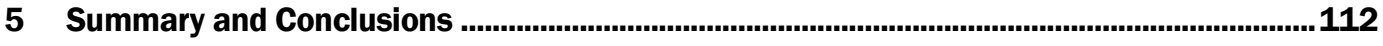

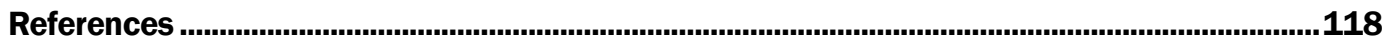

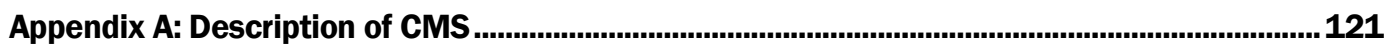

Appendix B: Description of BOUSS-2D ....................................................................................124

Appendix C: Details of Metocean Data for CDF4 ........................................................................134

Appendix D: Overtopping Estimates for Transects T1 to T10 .....................................................156

Report Documentation Page 


\section{Figures and Tables}

\section{Figures}

Figure 1-1. Google image and location of Confined Disposal Facility (CDF4), Buffalo, NY.................. 2

Figure 1-2. Existing CDF4 interior area with the bounding structures. .............................................

Figure 1-3. Wave overtopping Perimeter Dike of CDF4 during 25 November 2015 storm. ................. 4

Figure 2-1. Stony Point Breakwater (SPB) cross section. ...................................................................12

Figure 2-2. SPB, 12 July 2010. Note the extra row of stone placed along the crest. ........................12

Figure 2-3. Landward $304.8 \mathrm{~m}$ (1,000 ft) typical cross section of the South Entrance Arm

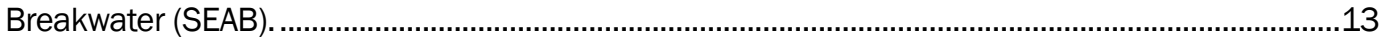

Figure 2-4. Landward $304.8 \mathrm{~m}$ (1,000 ft) typical cross section of the SEAB. ..................................13

Figure 2-5. SEAB, 12 July 2010. Note extra row of stone placed along crest....................................14

Figure 2-6. Perimeter Dike typical cross section. ............................................................................14

Figure 2-7. Interior dike location...............................................................................................15

Figure 2-8. Perimeter Dike crest repair cross section. .................................................................16

Figure 2-9. Sediment sample locations. ...................................................................................17

Figure 2-10. Location of NOAA, USGS, WIS, and ECCC coastal stations..........................................21

Figure 2-11. Class Angle 2 wave directions..................................................................................22

Figure 2-12. The 2-year wave and 2-year water level storm (Storm Condition 1). .............................30

Figure 2-13. The 20-year wave and 10-year water level storm (Storm Condition 2). ........................31

Figure 2-14. The 11 January 1982 storm (Storm Condition 3). .......................................................32

Figure 3-1. CMS-Wave parent grid domain (red box) and child grid domain (yellow box)..................35

Figure 3-2. CMS-Wave child grid domain (yellow box) with bathymetry contours..............................36

Figure 3-3. Parent grid; 7 spectral output (yellow dots) and 68 save locations (black dots)..............38

Figure 3-4. Child grid; 14 transect lines (T1 to T14) and 63 save locations (black dots)...................39

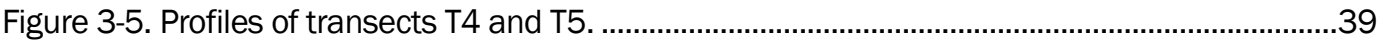

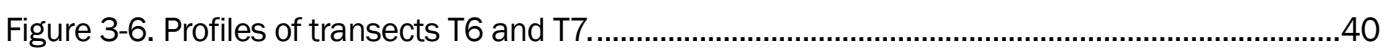

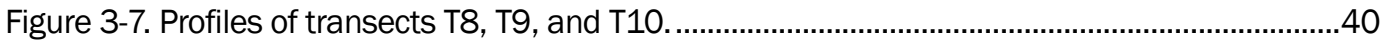

Figure 3-8. Profiles of transects T11, T12, T13, and T14 ............................................................. 41

Figure 3-9. Calculated wave field 11 January 1982; 12:00 GMT. ..................................................42

Figure 3-10. Calculated water level/current field 11 January1982; 12:00 GMT.............................43

Figure 3-11. Calculated wave/current field with permeable structures 11 January 1982;

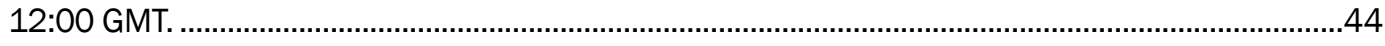

Figure 3-12. Calculated wave/current field with permeable dike 11 January 1982; 12:00 GMT..............44

Figure 3-13. Calculated wave/current field with impermeable dike 11 January 1982; 12:00 GMT.

Figure 3-14. Hourly and total wave overtopping rates along permeable structures (Storm Condition 1).

Figure 3-15. Hourly and total wave overtopping rates along permeable dike (Storm Condition 1) 
Figure 3-16. Hourly and total wave overtopping rates for impermeable structures (Storm Condition 1).

Figure 3-17. Hourly and total wave overtopping rates along permeable structures

(Storm Condition 2).

Figure 3-18. Hourly and total wave overtopping rates along permeable dike (Storm Condition 2)

Figure 3-19. Hourly and total wave overtopping rates for impermeable structures (Storm Condition 2)

Figure 3-20. Hourly and total wave overtopping rates along the permeable structures (Storm Condition 3).

Figure 3-21. Hourly and total wave overtopping rates along the dike for permeable dike (Storm Condition 3).

Figure 3-22. Hourly and total wave overtopping rates for impermeable structures (Storm Condition 3).

Figure 4-1. Perimeter Dike with (a) an arbitrary transect (red line), (b) elevation along thetransect (vertical and horizontal dimensions are in meters), and (c) design cross section of dike structure (vertically exaggerated).

Figure 4-2. B2D grid for existing CDF4 overlaid on a Google map image (origin of grid is at lower left corner).

Figure 4-3. Land (brown) and water (blue) areas in the B2D grid domain........................................58

Figure 4-4. Close-up of the CDF4 exterior area (vertical coordinate magnified)................................59

Figure 4-5. Close-up of the CDF4 interior (vertical coordinate magnified)........................................59

Figure 4-6. Recent photo showing the dike top section repaired crest along Leg 2 and Leg 3..............60

Figure 4-7. Typical view along Leg 4 and Leg 5 in September 2009. .................................................60

Figure 4-8. Transects T1 through T14 used for runup/overtopping calculations. ..............................61

Figure 4-9. Future year 2025 CDF 4 interior contours......................................................................62

Figure 4-10. Future year 2036 CDF 4 interior contours. ...................................................................62

Figure 4-11. Future raised elevation (red color) of Perimeter Dike at $20 \mathrm{ft}(6.2 \mathrm{~m})$........................63

Figure 4-12. Future raised Perimeter Dike without gaps..................................................................63

Figure 4-13. An example of overall wave height field.........................................................................65

Figure 4-14. View of sea surface elevation in the lake outside the front face of Perimeter Dike...............66

Figure 4-15. View of sea surface elevation from the interior of CDF4 .................................................66

Figure 4-16. Example of damping layers used in a test run..........................................................68

Figure 4-17. Wave height field of Storm Condition 1 over the model grid. ........................................ 71

Figure 4-18. Wave height field of Storm Condition 2 over the model grid. ........................................ 71

Figure 4-19. Wave height field of Storm Condition 3 (1982 storm) over the model grid..................72

Figure 4-20. A snapshot of Storm Condition 1 sea surface elevation around the CDF4...................73

Figure 4-21. A snapshot of Storm Condition 2 sea surface elevation around the CDF4..................73

Figure 4-22. A snapshot of Storm Condition 3 sea surface elevation in and around CDF4..............73

Figure 4-23. Progression of waves in the interior of CDF4 ................................................................. 74

Figure 4-24. A zoomed image of interior waves in south side of CDF4. ..............................................75

Figure 4-25. A closeup of interior waves on north side near the V-section of CDF4. ........................75

Figure 4-26. Overtopping at dike south and middle segments. ........................................................ 76 
Figure 4-27. Overtopping of dike south segments.......................................................................77

Figure 4-28. Overtopping at dike segment 4 (scene 1) ....................................................................77

Figure 4-29. Overtopping at dike segments 2, 3, 4, and 5 (scene 1)..............................................7

Figure 4-30. Overtopping at dike segment 5 (scene 1) ...............................................................78

Figure 4-31. Overtopping at dike segments 2 and 3 (scene 1)....................................................78

Figure 4-32. Overtopping at dike segment 5 (scene 2) ................................................................78

Figure 4-33. Overtopping at dike segments 2 , 3, and 4.................................................................79

Figure 4-34. Overtopping at dike segments 3 and 4 ..................................................................79

Figure 4-35. Overtopping at dike segment 5 (scene 3).............................................................79

Figure 4-36. Overtopping at dike segments 2 and 3 (scene 2)....................................................80

Figure 4-37. Overtopping at dike segments 4 and 5 (scene 1).....................................................80

Figure 4-38. Overtopping at dike segments 4 and 5 (scene 2).....................................................80

Figure 4-39. Overtopping at dike segment 4 (scene 2) .................................................................. 81

Figure 4-40. Overtopping at dike segments 2 and 5 .................................................................... 81

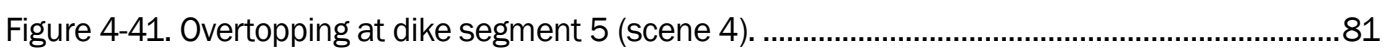

Figure 4-42. Overtopping at dike segments 2, 3, and 5.............................................................. 82

Figure 4-43. Overtopping at dike segments 2 , 4, and 5.............................................................

Figure 4-44. Overtopping at dike segments 4 and 5 (scene 3).....................................................82

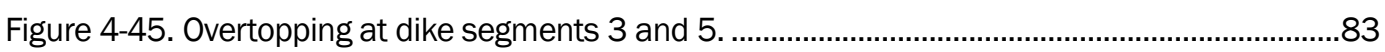

Figure 4-46. Overtopping at dike segments 2, 3, and 4 (scene 1)...............................................83

Figure 4-47. Overtopping at dike segments $2,3,4$, and 5 (scene 2). ............................................83

Figure 4-48. Overtopping at dike Segments 2, 3, and 4 (scene 2)..............................................84

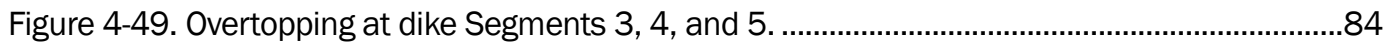

Figure 4-50. Wave height field of Storm Condition 1 (existing CDF4) ...........................................85

Figure 4-51. Wave height field of Storm Condition 2 (existing CDF4)..............................................85

Figure 4-52. Wave height field of Storm Condition 3 (existing CDF4)...............................................86

Figure 4-53. Arcs $A$ and $B$ in the interior V-section of CDF4 .......................................................86

Figure 4-54. Hs and MWL along arcs $A$ and $B$ for Storm Condition 2 (existing dike).........................8

Figure 4-55. Coupled B2D and B1D modeling using Arc3 as the wavemaker location for B1D................89

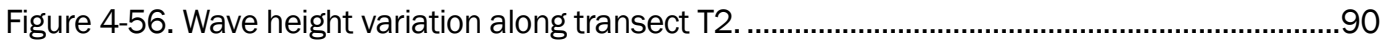

Figure 4-57. Overtopping rate and runup height variation along transect T2 (first 1,000 sec)...............91

Figure 4-58. Time variation of overtopping rate along transect T2 (entire simulation).....................91

Figure 4-59. Variation of wave height along transect T5...........................................................92

Figure 4-60. Variation of overtopping rate and runup height along transect T5 (first 1,000 sec)..............92

Figure 4-61. Time variation of overtopping rate along transect T5 (entire simulation).....................93

Figure 4-62. Wave height variation along transect T9. ……...........................................................93

Figure 4-63. Overtopping rate and runup height variation along transect T9 (first 1,000 sec).................94

Figure 4-64. Time variation of overtopping rate along transect T9 (entire simulation).....................94

Figure 4-65. Average overtopping rate for transects T1 to T10 (Storm Condition 2).........................95

Figure 4-66. Average overtopping rate for transects T1 to T10 (Storm Condition 1)........................96 
Figure 4-67. Average overtopping rate for transects T1 to T10 (Storm Condition 3) .96

Figure 4-68. Future Fill 2025 scenario with a small area of water in the V-section. 100

Figure 4-69. Storm Condition 1 results for Future Fill 2025; (a) wave surface elevation outside in the lake and (b) wave height field.

Figure 4-70. Wave height and water level variation along arcs A and B for Future Fill 2025 and existing CDF4, Storm Condition 1. 102

Figure 4-71. Snapshot of wave surface elevation for Future Fill 2025 (Storm Condition 1)......... 103

Figure 4-72. Snapshot of wave surface elevation for Future Fill 2025 (Storm Condition2).......... 103

Figure 4-73. Wave surface elevation snapshot for Future Fill 2036 (Storm Condition 1)............. 104

Figure 4-74. Lakeside buildup of wave surface elevation for Future Fill 2036 (Storm Condition 2). 105

Figure 4-75. Overtopping waves reaching the top of interior land surface (Storm Condition 2)........... 105

Figure 4-76. Water pockets developing at the top of interior land surface (Storm Condition 2)........... 106

Figure 4-77. Water pockets advancing on top of interior land surface (Storm Condition 2)........... 106

Figure 4-78. Water pockets increasing on top of interior land surface (Storm Condition 2)...........107

Figure 4-79. Continuous water coverage of front top section of interior land surface

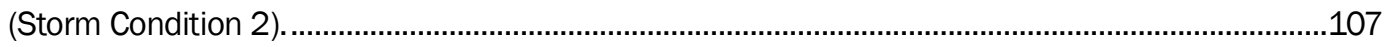

Figure 4-80. Image from B2D grid for the raised dike (gaps filled)................................................ 108

Figure 4-81. Hs and MWL with a raised dike (Storm Condition 3). .............................................. 109

Figure 4-82. Hs and MWL for existing dike (Storm Condition 3). ................................................... 109

Figure 4-83. Hs and MWL with a raised dike (Storm Condition 2)............................................... 110

Figure 4-84. Hs and MWL for existing dike (Storm Condition 2). ................................................... 110

Figure A-1. The CMS framework and its components................................................................. 121

Figure B-1. BOUSS-2D calculated wave-induced current field for Pillar Point Harbor, California. 128

Figure B-2. Calculated wave fields by (a) BOUSS-2D, and (b) CMS-Wave at Point Judith Harbor, Rhode Island, for incident wave from SSE.................................................. 129

Figure B-3. Wave propagation inside a bay................................................................................... 129

Figure B-4. Wave field around a detached breakwater................................................................... 130

Figure B-5. Waves, wave-induced current, and circulation near a reflective jetty of an inlet. ........ 130

Figure B-6. Wave-induced current field developed between two groins placed on a beach. ......... 131

Figure B-7. Multiple ships moving (in transit) in a harbor. ............................................................ 131

Figure B-8. BOUSS-2D domain for the Oyster Point Marina, California, entrance and east

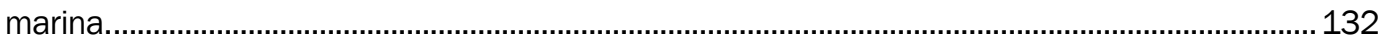

Figure B-9. BOUSS-2D grid for changes to entrance of Diversey Harbor, Michigan. ...................... 132

Figure B-10. BOUSS-2D runup/overtopping toolbox in SMS for a fringing reef application...........133

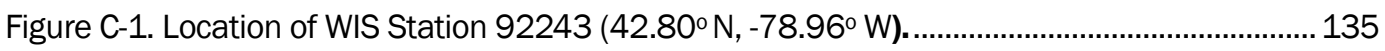

Figure C-2. Definition of Class Angle 2 wave directions................................................................. 136

Figure C-3. Wave height vs. return period........................................................................................ 137

Figure C-4. Wave period vs. wave height relation. ......................................................................... 138

Figure C-5. Three storms used for 2-year wave height hydrograph (peaks aligned)........................141

Figure C-6. Three storms used for 20-year wave height hydrograph (peaks aligned)....................141 
Figure C-7. Three storms used for 2-year wave period hydrograph (peaks aligned)....................... 142

Figure C-8. Three storms used for 20-year wave period hydrograph (peaks aligned).................... 142

Figure C-9. Wave height vs.wind speed at constant wind direction of 247 deg............................ 143

Figure C-10. Lake Erie water elevation at Buffalo, NY (Return Period relation)............................... 144

Figure C-11. Three storms used to create the 2-year water level hydrograph................................ 145

Figure C-12. Three storms used to create the 10-year water level hydrograph............................... 146

Figure C-13. Time series data for the 2-year wave and 2-year water level storm........................... 149

Figure C-14. Time series data for the 20-year wave and 10-year water level storm....................... 152

Figure C-15. Time series data for the 11 January 1982 storm. ..................................................... 155

Figure D-1. Variation in wave height along T1 ................................................................................ 156

Figure D-2. Variation of overtopping rate and runup height along T1 (first 1,000 sec). ................ 157

Figure D-3. Temporal variation of overtopping rate along T1 (entire simulation)........................... 157

Figure D-4. Wave height variation along T3.................................................................................. 158

Figure D-5. Overtopping rate and runup height variation along T3 (first 1,000 sec)..................... 158

Figure D-6. Time variation of overtopping rate along T3 (entire simulation). ................................... 159

Figure D-7. Wave height variation along T4. ............................................................................... 160

Figure D-8. Overtopping rate and runup height variation along T4 (first 1,000 sec).................... 161

Figure D-9. Time variation of overtopping rate along T4 (entire simulation). ................................ 161

Figure D-10. Wave height variation along T6................................................................................. 162

Figure D-11. Overtopping rate and runup height variation along T6 (first 1,000 sec)................. 162

Figure D-12. Time variation of overtopping rate along T6 (entire simulation)............................... 163

Figure D-13. Wave height variation along T7............................................................................... 163

Figure D-14. Overtopping rate and runup height variation along T7 (first 1,000 sec).................. 164

Figure D-15. Time variation of overtopping rate along T7 (entire simulation)................................ 164

Figure D-16. Wave height variation along T8................................................................................. 165

Figure D-17. Overtopping rate and runup height variation along T8 (first 1,000 sec)................... 165

Figure D-18. Time variation of overtopping rate along T8 (entire simulation)................................ 166

Figure D-19. Wave height variation along T10................................................................................ 166

Figure D-20. Overtopping rate and runup height variation along T10 (first 1,000 sec)................ 167

Figure D-21. Time variation of overtopping rate along T10 (entire simulation)............................. 167

\section{Tables}

Table 2-1. Mean monthly and annual discharge for the Niagara River at Buffalo, NY......................19

Table 2-2. Buffalo River average annual discharge...........................................................................20

Table 2-3. List of selected storm wave events from WIS Station 92243............................................23

Table 2-4. Class Angle 2 recurrence of storm wave heights and associated wave period. ................23

Table 2-5. Water levels at NOAA Station 9063020 for several recurrence intervals.........................24

Table 2-6. Hourly data for the 2-year wave and 2-year water level storm (Storm Condition 1)................25

Table 2-7. Hourly data for the 20-year wave and 10-year water level storm

(Storm Condition 2) 
Table 2-8. Hourly data for the 11 January 1982 storm (Storm Condition 3)...................................28

Table 3-1. Maximum wave overtopping rate and total volume (Storm Condition 1).........................51

Table 3-2. Maximum wave overtopping rate and total volume (Storm Condition 2)..........................51

Table 3-3. Maximum wave overtopping rate and total volume (Storm Condition 3)..........................51

Table 4-1. B2D input incident wave parameters and water levels. ...................................................64

Table 4-2. Wave height and overtopping rate statistics for transects T1 through T10

(Storm Condition 2) ...........................................................................................................97

Table 4-3. Wave height and overtopping rate statistics for transects T1 through T10

(Storm Condition 1) ............................................................................................................................98

Table 4-4. Wave height and overtopping rate statistics for transects T1 through T10

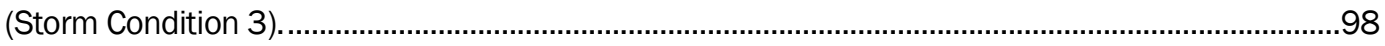

Table C-1. Storm list for wave heights greater than $4 \mathrm{~m}$ (13.1 ft) (1979-2012). ............................ 136

Table C-2. Descriptive statistics for storm wave direction. ............................................................... 138

Table C-3. Class Angle 2 recurrence interval for WIS Sta 92243...................................................... 139

Table C-4. Return period for lake level..................................................................................... 144

Table C-5. Hourly data for 2-year wave and 2-year water level storm. ............................................ 146

Table C-6. Hourly data for 20-year wave and 10-year water level storm........................................ 150

Table C-7. Hourly data for the 11 January 1982 storm...................................................................... 152 


\section{Preface}

This study was performed during the time period December 2015 to December 2016 by the Coastal and Hydraulics Laboratory (CHL) of the U.S. Army Engineer Research and Development Center (ERDC), at the request of the U.S. Army Corps of Engineers, Buffalo District.

The U.S Army Corps of Engineers (USACE) Coastal Inlets Research Program (CIRP) provided partial support for this study through Wave Structures Work Unit $280 \mathrm{H} 46$ for improvement of wave processes modeling over the dikes that are important at this project site. CIRP is administered at CHL under the USACE Navigation Research, Development, and Technology Transfer (RD\&T) Program. At the time this effort was conducted, Mr. Jeffrey A. McKee was the USACE Headquarters Navigation Business Line Manager overseeing the CIRP. Mr. W. Jeff Lillycrop, CHL, was the ERDC Technical Director for Civil Works and Navigation RD\&T. Mr. Charles E. Wiggins, CHL, was the ERDC Associate Technical Director for Navigation, and Dr. Julie Rosati was CIRP Program Manager.

During the study period, the general administrative supervision was provided by Mr. James Gutshall, Chief, Harbors, Entrances, and Structures Branch; Dr. Jackie Pettway, Chief, Navigation Division; Mr. Jeffrey R. Eckstein, Deputy Director of CHL; and Mr. José E. Sánchez, the Director of CHL.

At the time of publication of this report, COL Bryan S. Green was Commander of ERDC, and Dr. David W. Pittman was the ERDC Director. 


\section{Unit Conversion Factors}

\begin{tabular}{|l|c|l|}
\hline Multiply & By & To Obtain \\
\hline acres & $4,046.873$ & square meters \\
\hline cubic yards & 0.7645549 & cubic meters \\
\hline feet & 0.3048 & meters \\
\hline knots & 0.5144444 & meters per second \\
\hline miles per hour & 0.44704 & meters per second \\
\hline miles (U.S. nautical) & 1.852 & kilometers \\
\hline miles (U.S. statute) & 1.609347 & kilometers \\
\hline
\end{tabular}




\section{Introduction}

\subsection{Background and study area}

The Confined Disposal Facility \#4 (CDF4) is located in Lake Erie at Buffalo, NY, on the lake side of the South Entrance Channel to Buffalo Harbor (Figure 1-1). The boundaries of the disposal area include two breakwaters on the east side and one curved dike on the west side (Figure 1-2). The South Entrance Arm Breakwater (SEAB) and Stony Point Breakwater (SPB) define the southern boundary of the South Entrance Channel to the Buffalo Harbor, New York, isolating the $\mathrm{CDF}_{4}$ from the entrance channel and harbor complex. A curved dike known as the Perimeter Dike (consisting of Legs 1 through 5) isolates the disposal area from the lake side. The SPB was constructed first in 1897, the SEAB was added as an extension in 1936, and finally, the Perimeter Dike was added in 1977 to develop a fully contained confined disposal area defined by these three structures. The SEAB is approximately 2000 feet (ft) long, has a crest elevation of $+16 \mathrm{ft}$ Low Water Datum (LWD), the SPB is $1600 \mathrm{ft}$ long with a crest elevation of $+12 \mathrm{ft}$, and the curved dike is $3700 \mathrm{ft}$ long and has crest elevation of $+12 \mathrm{ft}$. The Perimeter Dike protecting the lakeside of $\mathrm{CDF}_{4}$ is a curved stone structure that consists of five legs (segments) and connects the north end of the SEAB to the south shoreline of Lake Erie (Figures 1-1 and 1-2).

Periodic overtopping of the Perimeter Dike occurs during storms. The wave overtopping raised concerns that waves getting into the Confined Disposal Facility (CDF) interior could cause contaminated sediments to mobilize and possibly exit the CDF. Physical evidence (accumulation of woody debris along the SEAB and SPB) and a past claim by a local marina owner of woody debris exiting the CDF east side at a time when there was less material in the CDF, prompted the U.S. Army Corps of Engineers, Buffalo District (LRB) to create a low, temporary berm in front of the SPB using dredge material from CDF4. Additionally, the LRB, pursued a modeling effort to simulate significant storms with the accompanying persistent and excessive overtopping because of the potential for these overtopping events mobilizing sediment over the SEAB to the south harbor entrance, navigation channel, and harbor complex.

Though there are anecdotal claims and debris indicating possible sediment movement, there has been no evidence of any significant increase in the existing contamination levels outside the $\mathrm{CDF}_{4}$. Consequently, flow and 
Figure 1-1. Google image and location of Confined Disposal Facility (CDF4), Buffalo, NY.

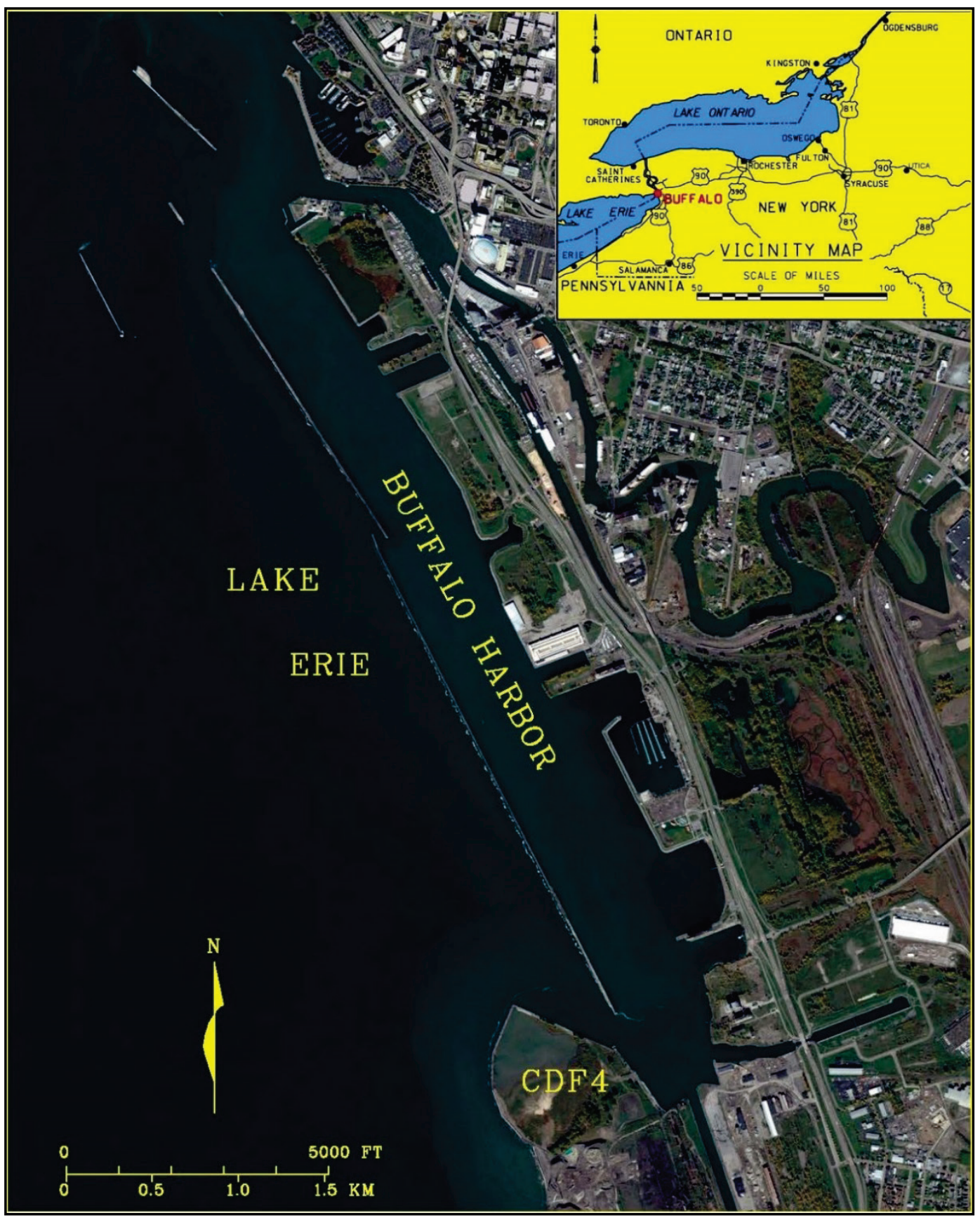

sediment transport modeling were not considered in the present study; rather, the focus was on modeling waves inside and outside the $\mathrm{CDF}_{4}$ and providing estimates of wave overtopping caused by extremal events. Overtopping estimates for different storms and water levels along the segments of the curved dike can be used for potential solutions to minimize/prevent movement of contaminated sediments out of the CDF4. Modeling results for two future fill scenarios (Future Fill 2025 and 
Figure 1-2. Existing CDF4 interior area with the bounding structures.

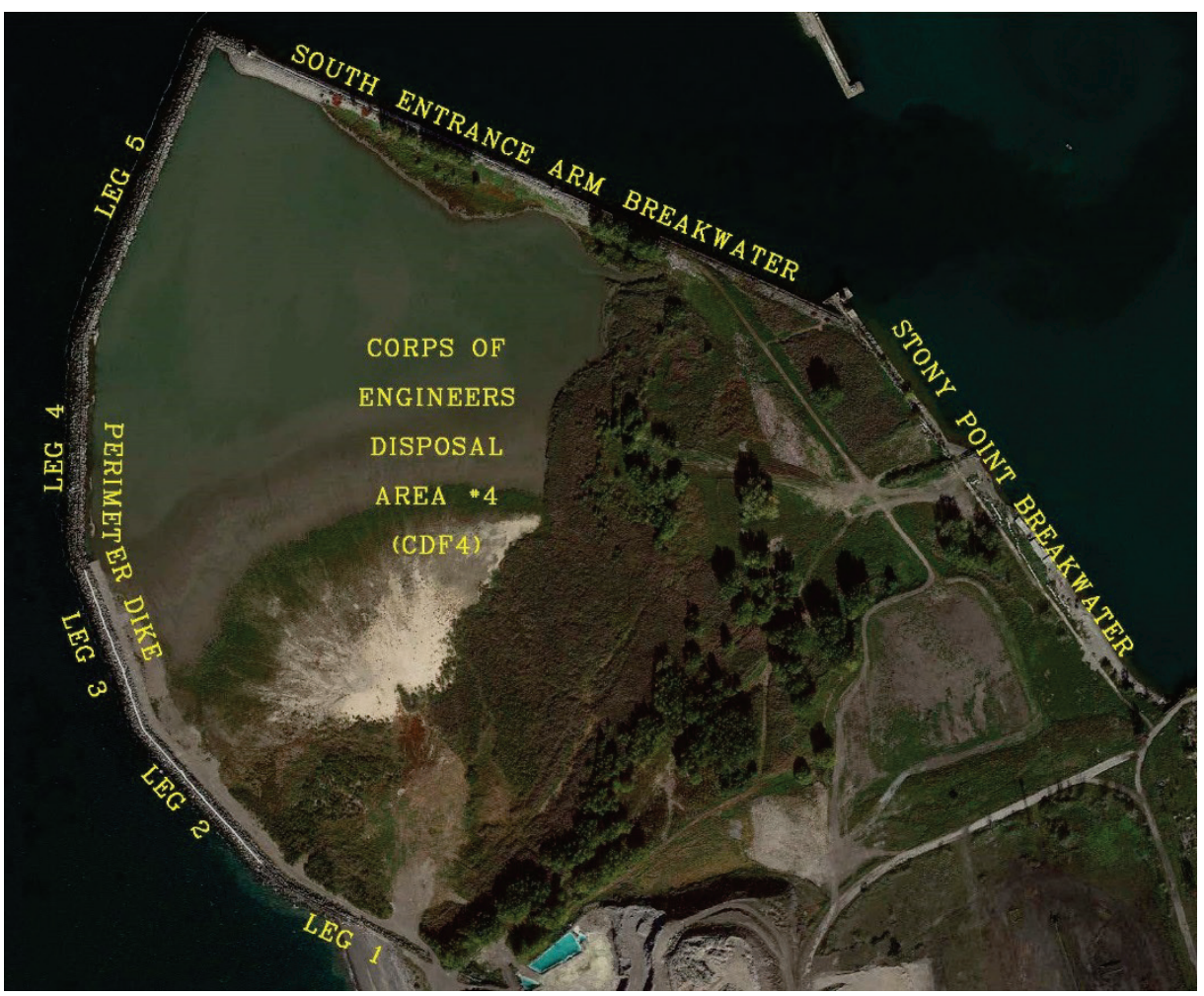

Future Fill 2036) are provided. Wave runup/overtopping estimates of the existing CDF system were developed by modeling wave processes in the exterior and interior areas of the $\mathrm{CDF}_{4}$ using two classes of wave models: CMS-Wave, a spectral wind-wave generation, growth and transformation model, and a fully nonlinear Boussinesq type wave model (BOUSS-1D/2D). Appendices A and B provide summary information about these models, their features, and example applications. Detailed information about these models is available from publications listed in the References section of this report (Demirbilek et al. 2016, 2015a,b; Demirbilek and Rosati 2011; Lin et al. 2011a,b).

\subsection{Condition of existing Confined Disposal Facility (CDF)}

The Perimeter Dike structure is composed of multiple stone layers, with a filter stone layer to reduce the permeability of the dike, and a row of steel sheet pile that extends downward from the dike crest $(+15 \mathrm{ft}$ Low Water Datum [LWD]) through the filter stone to an elevation of approximately $-9 \mathrm{ft}$ LWD. Recent visual inspections indicated that the stones along the outer perimeter of the dike have deteriorated, moved, and settled. Crest lowering, stone cracking, and movement were apparent along the entire rubble mound portion of the containing walls. 
In addition to these acute stone degradations near the center of dike, deterioration and movement of the large armor away from the dike crest were observed. This allowed ice-jacking of the steel sheet piles to occur at various locations along the dike, compromising the dike's intended function at those locations. Ice-jacking occurs when water freezes and causes upward lifting of the sheet piles and surface stones with gaps in between. This was remedied by cutting the piles to reduce loads and by grouting those locations. In 2016, the crest of the structure along Legs 2 and 3 was repaired by replacing the damaged crest stones with a cast-in-place block and several new armor stones. With a reduced (low) crest elevation of the perimeter structure, waves can more readily overtop the Perimeter Dike even during medium storms, propagate through the interior open water inside CDF4, and may potentially exit the opposite side into the South Entrance Channel. There are no field data available on the frequency and severity of overtopping occurring or extent of the potential loss of material from the interior of CDF4 system. These concerns are the focus of the present investigation. Figure 1-3 is a photograph looking lakeward (to the west) from the dike interior that shows wave overtopping during a storm on 25 November 2015 at 09:45 a.m. Eastern Standard Time.

Figure 1-3. Wave overtopping Perimeter Dike of CDF4 during 25 November 2015 storm.

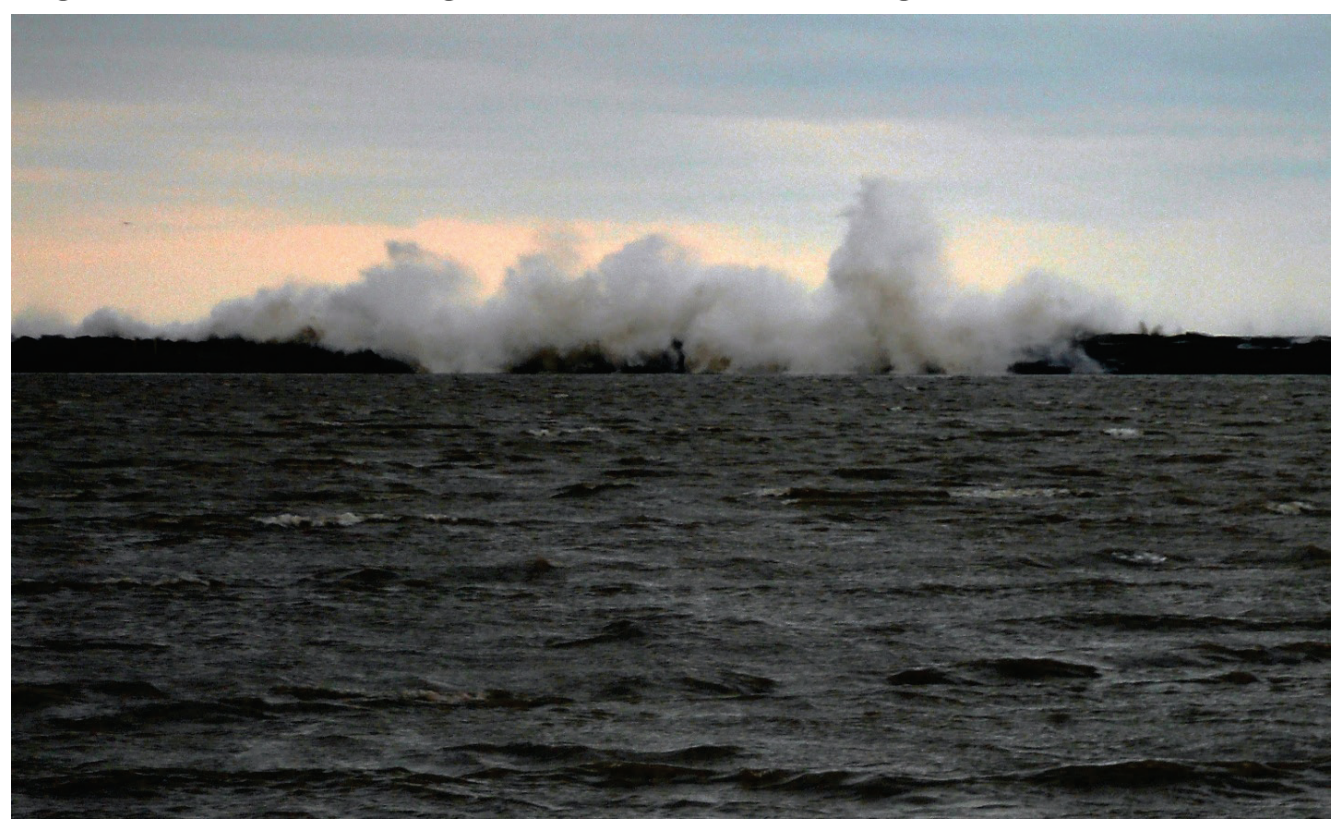

\subsection{Environmental forcings}

Storm waves developing in Lake Erie are the primary source of wave overtopping affecting the $\mathrm{CDF} 4$ system. The Perimeter Dike shelters the interior of $\mathrm{CDF}$ from the incident wind-waves reaching the project site. Lake 
Erie is the eleventh largest lake in the world by surface area with a width of 92 kilometers $(\mathrm{km})(57$ miles) and average depth of 19 meters $(\mathrm{m})(57 \mathrm{ft})$ and length of $388 \mathrm{~km}$ (241 miles) (GLIN 2017). CDF4 is at the eastern end of the lake and situated along the longest fetch (from due southwest to northeast) and typical storm direction. This can result in the largest waves and wind setup conditions which can be ranging up to $2.4 \mathrm{~m}(8 \mathrm{ft})$ in elevation reaching the $\mathrm{CDF}_{4}$ site. The closest water level gage is within Buffalo Harbor (5.3 km [3.3 miles] from the CDF4 site) and wind information at the Buffalo airport (19.2 km [11.9 miles]). There were no field gauges providing wave or current measurements at or near the CDF4. Consequently, meteorological and oceanographic forcings for this study were obtained from existing data sources. These include the USACE Wave Information Study (WIS) wave hindcast data, the National Oceanic and Atmospheric Agency (NOAA) buoys and gauges available in Lake Erie, the Great Lakes Observing System, and the Great Lakes Coastal Forecasting System (GLCFS).

The water level, wind, and wave conditions used in this study were developed jointly by the LRB and the U.S. Army Engineer Research and Development Center (ERDC), Coastal and Hydraulics Laboratory (CHL), team members from these sources. The bathymetry and structural data used in modeling were provided by LRB. Incident waves are not affected by the open lake bathymetry until they propagate into the proximity of CDF4. Waves can strongly interact with the structures protecting the $\mathrm{CDF}_{4}$, and overtopping waves move inside the disposal area flooding portions of the $\mathrm{CDF}_{4}$ (which is now largely filled-in/land). The water depth over the lake decreases as waves approach $\mathrm{CDF}_{4}$ and the entrance channel before moving into the harbor interior to interact with the protective structures and shorelines. Breaking waves over the protective structures and inside and outside shallow areas generate wave-induced currents, which can adversely affect navigation into/out of the harbor as well as potentially transport littoral sediments inside the disposal area, into the channel and harbor, and other adjacent areas to the CDF4.

\subsection{Study plan}

\subsubsection{Purpose}

There was no information available about prior field data collection or numerical modeling studies conducted for $\mathrm{CDF}_{4}$. Because the focus of the present study was limited to wave modeling at $\mathrm{CDF}_{4}$ for providing wave overtopping estimates. Assessing potential impacts on navigation, 
hydrodynamics, and sediment transport issues were not considered in this study. Combined wave and current modeling may be necessary in the future to ascertain long-term behavior of $\mathrm{CDF}_{4}$ as a system to waves, including potential effects of hydrodynamics and sediment processes inside and outside the CDF4.

The most recent bathymetric, wind, and wave data available for this site were used in the numerical modeling investigation. Together with the bathymetric data, it is the magnitude and direction of forcing conditions (winds, waves, water levels) used that determine the impacts of waves on exterior and interior conditions of the $\mathrm{CDF}_{4}$ and how overtopping waves might affect the adjacent navigation South Entrance Channel that connects to the Buffalo Harbor complex. The ultimate goal of this numerical modeling study is to advise the LRB on estimates of the potential for wave overtopping of the existing CDF4 system.

\subsubsection{Approach}

As noted in the previous section, it was necessary to use two wave models for developing estimates of waves and wave runup/overtopping for the CDF4 site. CMS-Wave, a spectral wind-wave transformation model for open coast and nearshore applications, was used for regional-scale, deep-water wave transformation and estimating wave overtopping. B2D, a Boussinesqtype nonlinear, time-domain wave model developed specifically for modeling waves in nearshore applications including wave-structure interactions and navigation studies (e.g., channel widening, deepening, infrastructure modifications to ports, harbors, and marinas), was used for developing wave runup and also providing overtopping estimates. B2D was also used to model the flooding over the interior of CDF4 under existing conditions, two future fill scenarios (Future Fill 2025 and Future Fill 2036), and a raised Perimeter Dike scenario. The latter three scenarios were not in the scope of study and were added later at the request of LRB study team.

A number of additional Research and Development (R\&D) refinements were made and adapted to CMS-Wave to develop input conditions to address this project's specific needs. These project-specific improvements for the model's predictive capabilities were funded by the Coastal Inlets Research Program (CIRP), including (a) analysis of wave hindcast, wind, and water level data for simulations of storms and non-storm waves in Lake Erie, (b) an investigation of the sensitivity of modeling results to the location of the WIS station far from project site in the Lake Erie used for incident wave 
conditions to force CMS-Wave, (c) development of wave runup/overtopping formulas and pre- and post-processing analysis codes to perform wave runup/overtopping calculations along selected transects (arcs and lines), (d) development of Fortran and Matlab programs to provide wave input conditions as wave parameters (height, period, direction) at the depth contour of $10 \mathrm{~m}(33 \mathrm{ft})$ and at $100 \mathrm{~m}(330 \mathrm{ft})$ distance seaward of the toe of the Perimeter Dike, (e) development of Fortran and Matlab utilities for analyses of two model results in time and frequency domains, and (f) development of Matlab codes for comparison of wave overtopping estimates from solution files of both models and empirical formulas from the Coastal Engineering Manual (CEM) (Demirbilek and Vincent 2015) and European Overtopping (Eurotop) manual (http://www.overtopping-manual.com).

The long-term wave climate in Lake Erie was required to perform this numerical modeling study. Deep-water wave data for this study were based on the Wave Information Studies (WIS hindcast time series (1980-2011) available for Lake Erie. Incident deep-water waves were transformed to the project site using CMS-Wave. The CMS-Wave model results were used as input wave forcing at the boundary of the B2D model domain for calculating wave runup/overtopping at CDF4. CMS-Wave results were also used for overtopping estimates for comparison to the Eurotop and B2D estimates.

The LRB conducted an analysis of predominant wind, water level, and wave conditions in Lake Erie to determine representative design storms for (1) the 2-year wave and 2-year water level and (2) the 20-year wave and 10-year water level for wave modeling and wave runup/overtopping estimates at the $\mathrm{CDF} 4$. The goal of analysis was to provide design, repair, and maintenance conditions for present and future remedial actions for the CDF system. The return period events were investigated to provide LRB design wave and overtopping estimates for the next phase of this project (e.g., future Operations and Maintenance [O\&M] studies involving structural repairs, environmental studies).

The present study focused only on modeling of waves inside and outside the $\mathrm{CDF}_{4}$ and developing of estimates of overtopping for significant storm events and water levels in this portion of the Lake Erie. Flow and sediment transport modeling inside and outside the $\mathrm{CDF} 4$ were not considered in this study. However, it was assumed that if flow out of the CDF were observed, it was accompanied by suspended sediment. Overtopping estimates are provided for different storms and water levels along the segments of 
Perimeter Dike for potential future solutions to minimize/prevent contaminated sediment from exiting the $\mathrm{CDF} 4$. Results from a preliminary investigation are also provided concerning the two $\mathrm{CDF}_{4}$ interior future fill scenarios (Future Fill 2025 and Future Fill 2036) and raising the Perimeter Dike to assist LRB for planning future remedial actions that may be necessary. The remainder of this report documents details of the modeling performed, with a discussion of study results and recommendations.

The wave processes in exterior and interior areas of the CDF4 near Buffalo, $\mathrm{NY}$, were investigated to determine wave runup/overtopping estimates for the existing $\mathrm{CDF}_{4}$ system using two different classes of wave models. Boussinesq (BOUSS-2D or B2D) and the Coastal Modeling System (CMS)Wave used are part of the CIRP modeling technology. CMS-Wave, a spectral wind-wave generation, growth and transformation model was used to develop wave and water level estimates at the CDF4. This model also provided inputs to a fully nonlinear Boussinesq type wave model (BOUSS2D). These two models were used to quantify the impacts of waves on the CDF4 and address related concerns to navigation and the environment. CMS-Wave was used to transform deep water waves to the project site and to develop wave estimates in the existing harbor and for alternatives. Both one-dimensional (1D) and two-dimensional (2D) versions of Boussinesq model (B1D and B2D) were used to investigate the details of wave runup/overtopping of the $\mathrm{CDF} 4$ structures. B2D was also used for flooding over the interior of CDF4 for Future Fill 2025 and Future Fill 2016 scenarios.

The combination of the two wave models was necessary to address the needs of this project. In addition to differences between the models' theoretical and numerical formulations, different spatial extents and resolutions of model grids were used to properly model waves for the needs of this project. Despite the stated differences between the two different classes of wave models, where possible, general comparisons of two models were made, and potential causes of significantly different estimates were examined and resolved. The use of this two-model approach was helpful in checking and identifying potential errors in setting up models and examining/comparing results.

CMS-Wave and both B1D/B2D were applied using the default model settings recommended in their technical reports (Lin et al. 2008; Nwogu and Demirbilek 2001). This approach was used because no wave or CDF 
interior water level field data were available in the study area to calibrate and validate models. The use of default settings in both models has been demonstrated in other harbor and inlet applications with similar structures and wave and water conditions (Demirbilek et al. 2016, 2015a, 2015b, 2009, 2008, 2007a, 2007b, 2007c; Lin and Demirbilek 2012, 2005; Lin et al. 2011a, 2011b, 2008).

Estimates of wave parameters (height, period, and direction) from B2D and CMS-Wave models can be used for operation and design projects at or near the $\mathrm{CDF} 4$ project site. These estimates may be combined with experiences of $\mathrm{CDF} 4$ monitoring studies to determine infrastructure modifications required for prevention and/or minimization of potential impacts of the contaminated material that can be transported out of the $\mathrm{CDF}_{4}$ interior as the disposal area gets filled in over the next 2 decades. Proper management of the CDF4 and its impacts on navigation will help to increase operational efficiency of Buffalo Harbor (i.e., improving both access and utilization of the harbor). The B2D modeling results for two future fill scenarios also helps LRB to assess potential overtopping of the SEAB that would require certain adjustments to the elevation of the breakwater or immediate vicinity. Overall, numerical modeling predictions from this study will help LRB to determine remedial actions that could prevent/reduce wave overtopping of the $\mathrm{CDF}_{4}$ and its potentially harmful consequences to sensitive areas nearby (e.g., Buffalo Harbor complex).

\subsubsection{Study tasks}

The main tasks of this study were (1) define regional meteorological and oceanographic conditions (e.g., winds, waves, water levels), (2) assemble bathymetric, shoreline, and structural data for interior and exterior regions of the $\mathrm{CDF}_{4}$, (3) calculate waves outside and inside of the existing $\mathrm{CDF}_{4}$, (4) investigate details of wave runup/overtopping of the dike protecting the $\mathrm{CDF} 4$, and (5) analyze model results and summarize key findings in a technical report. Although not in the scope of study, an additional task was added to investigate the possibility of waves inside the CDF4 overtopping the SEAB if and when (1) the interior is mostly or completely filled in with the contaminated material and (2) the Perimeter Dike is raised.

Last, this was the first wave overtopping study performed using the combination of CMS and B2D/B1D models. The tasks were broad and challenging, which required expansion of existing and development of additional model capabilities necessary to produce estimates of wave runup 
and overtopping by the coupled modeling system. The study also required development of a number of post-processing analysis capabilities for producing the statistical estimates of wave runup and overtopping rates and comparison to international standards in Eurotop manual. The CIRP partially funded the research activities related to improvement of the numerical models and analysis capabilities. 


\section{Data for the Study}

\subsection{Bathymetry and coastline data}

Coastline digital data for this study were extracted from the National Geophysical Data Center (http://www.ngdc.noaa.gov/mgg/shorelines/) and a georeferenced image file downloaded from Google Earth 5.0 (http://earth.google.com).

The bathymetry data used in this study were obtained from various sources covering the harbor, land, nearshore, and offshore area, but no new survey data were acquired for this project. The harbor area bathymetry, including the interior, channel, structures (breakwater, dike, jetty, seawall, etc.), and shoreline data, were based on FEMA 2008 lidar data collection and LRB September 2015 surveys. All datasets were converted to the Lake Erie's LWD, which is $173.5 \mathrm{~m}(569.2 \mathrm{ft})$ above the International Great Lakes Datum (IGLD) 1985.

\subsection{Structure data}

CDF4 was created by using the existing SPB and South Entrance Arm Breakwaters SEAB built over a century ago and by building a new Perimeter Dike in 1977 to create the enclosure.

The SEAB was originally constructed in 1897-98 and was composed of stone-filled timber cribs resting on a trench excavated down to bedrock filled with gravel. In 1923-24, the timber crest was removed and replaced with cut stone along the crest and lakeside (Figure 2-1). The crest elevation expressed in modern terms was rebuilt to $+3.66 \mathrm{~m} \mathrm{LWD} \mathrm{(+12} \mathrm{ft} \mathrm{LWD)} \mathrm{with}$ a lakeside stone slope of $1 \mathrm{~V}: 1.5 \mathrm{H}$. Figure 2-2 presents a contemporary photo of the SPB taken from the Buffalo Harbor interior channel side. Note the addition of cut stone blocks lining the crest of the structure and the vegetation. A century ago, the far side was the open water of the lake, but with the creation of the $\mathrm{CDF}$, that has changed due to the placement of dredged material. 
Figure 2-1. Stony Point Breakwater (SPB) cross section.

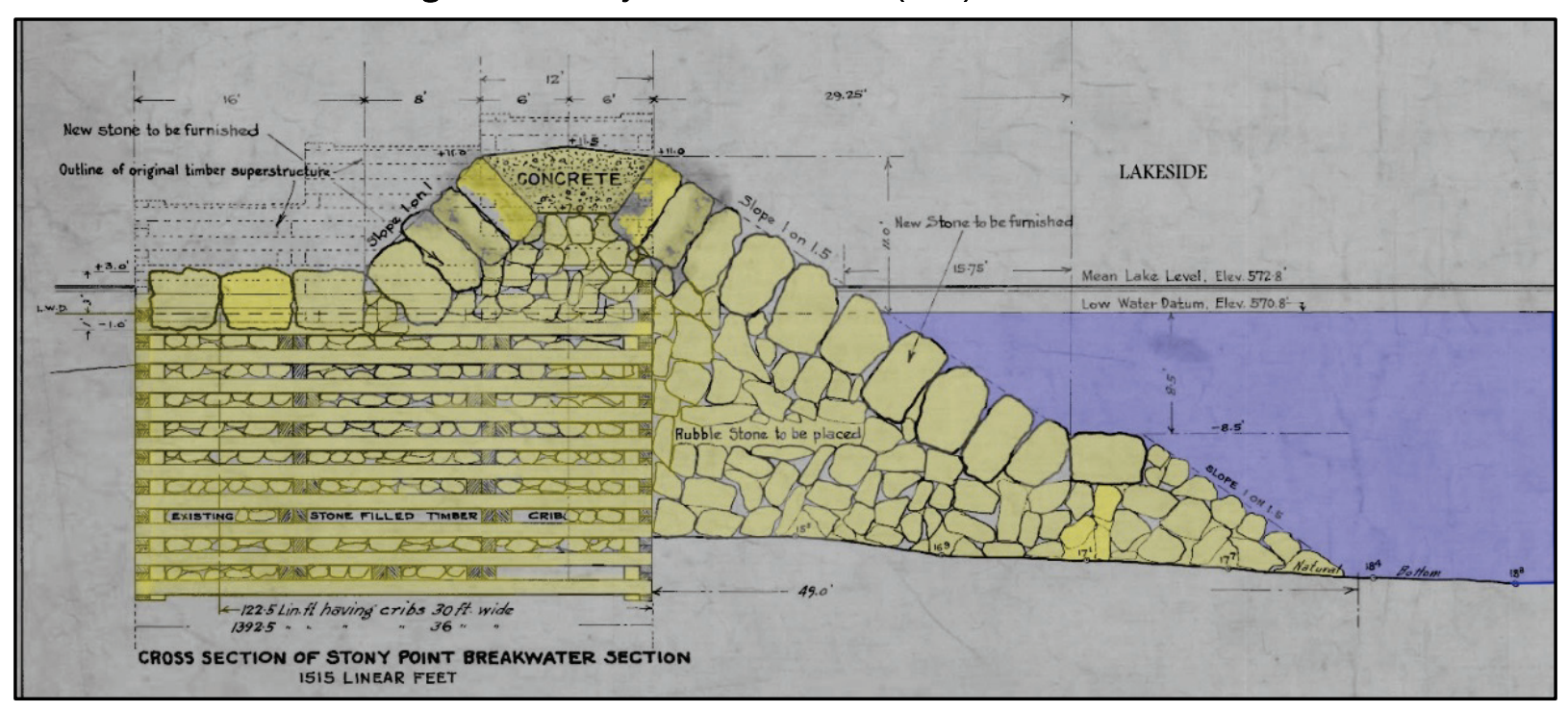

Figure 2-2. SPB, 12 July 2010. Note the extra row of stone placed along the crest.

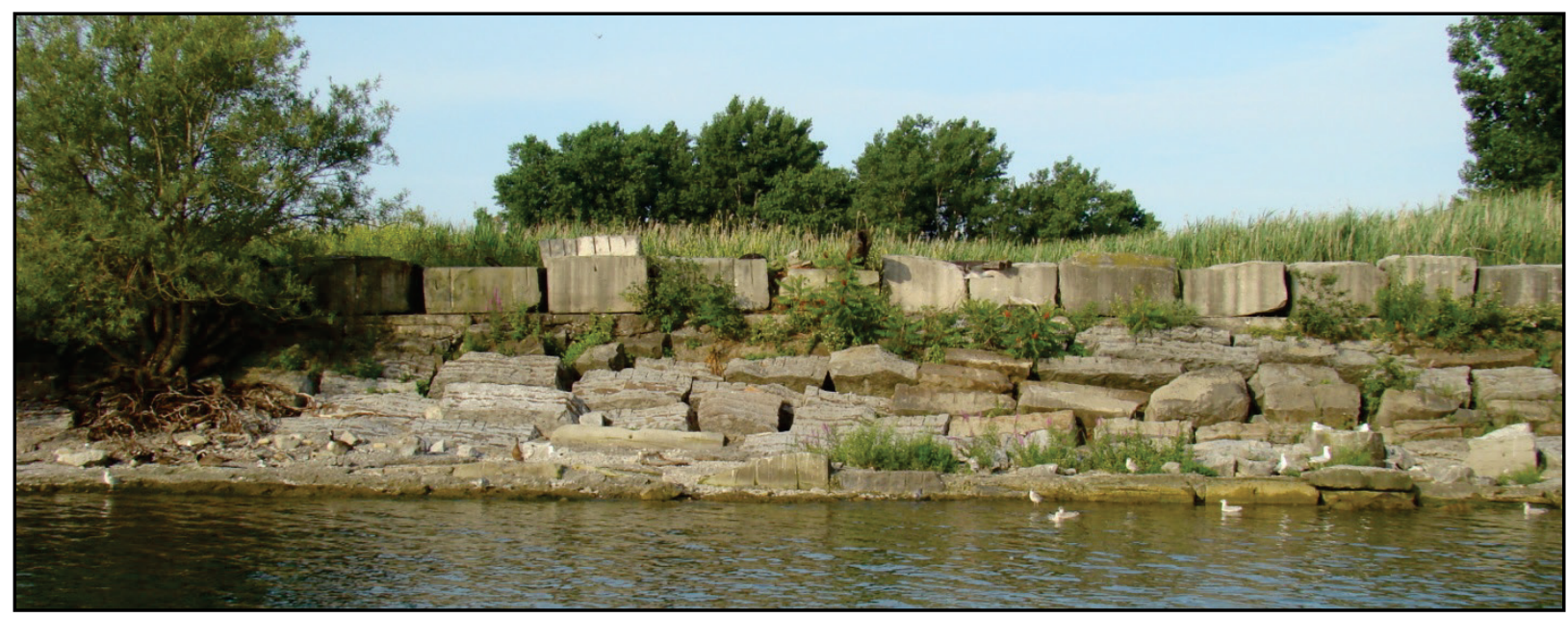

The SEAB originally extended $304.8 \mathrm{~m}(1,000 \mathrm{ft})$ in a northwest direction from the SPB, was built of stone with a crest elevation in modern terms of +4.4 $\mathrm{m}$ LWD (+14.5 ft LWD), and was completed in 1911 (Figure 2-3). 
Figure 2-3. Landward $304.8 \mathrm{~m}$ (1,000 ft) typical cross section of the South Entrance Arm Breakwater (SEAB).

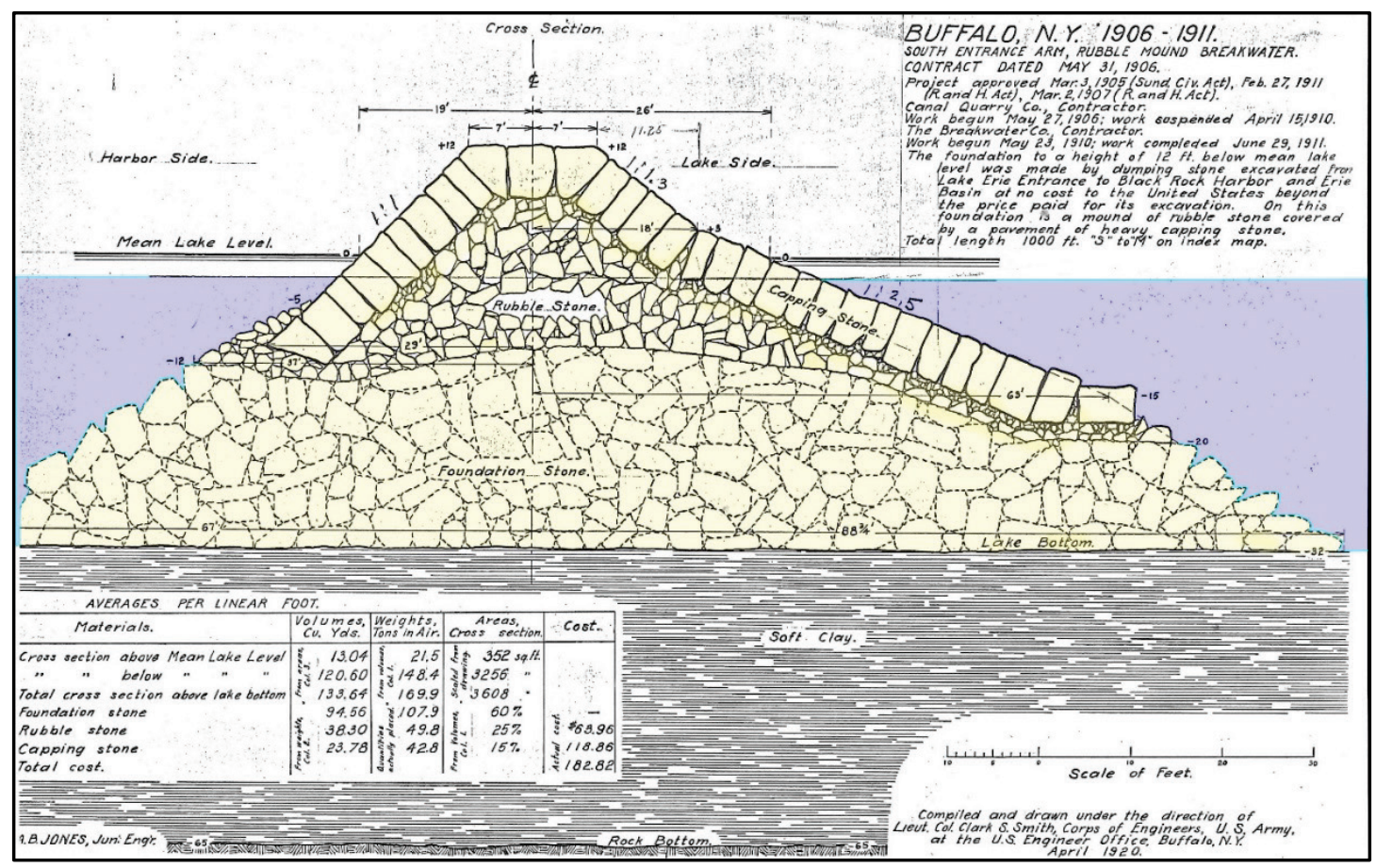

The SEAB was extended another $304.8 \mathrm{~m}$ (1,000 ft) during the period November 1933 to November 1935. The breakwater has a rubble core and cut stone ashlar facing that slopes at $1 \mathrm{~V}: 1.5 \mathrm{H}$ on both sides. A $3 \mathrm{~m} \mathrm{(10} \mathrm{ft)}$ wide deck stone at the crest of the structure was originally set at $+4.3 \mathrm{~m}$ LWD (+14.1 ft LWD). Figure 2-4 presents the original cross section, and Figure 2-5 presents a contemporary photograph taken from the Buffalo Harbor interior channel side. Note that a row of cut stone has also been added to this structure, raising the original crest to approximately $+4.9 \mathrm{~m}$ LWD (+16 ft LWD).

Figure 2-4. Landward $304.8 \mathrm{~m}(1,000 \mathrm{ft})$ typical cross section of the SEAB.

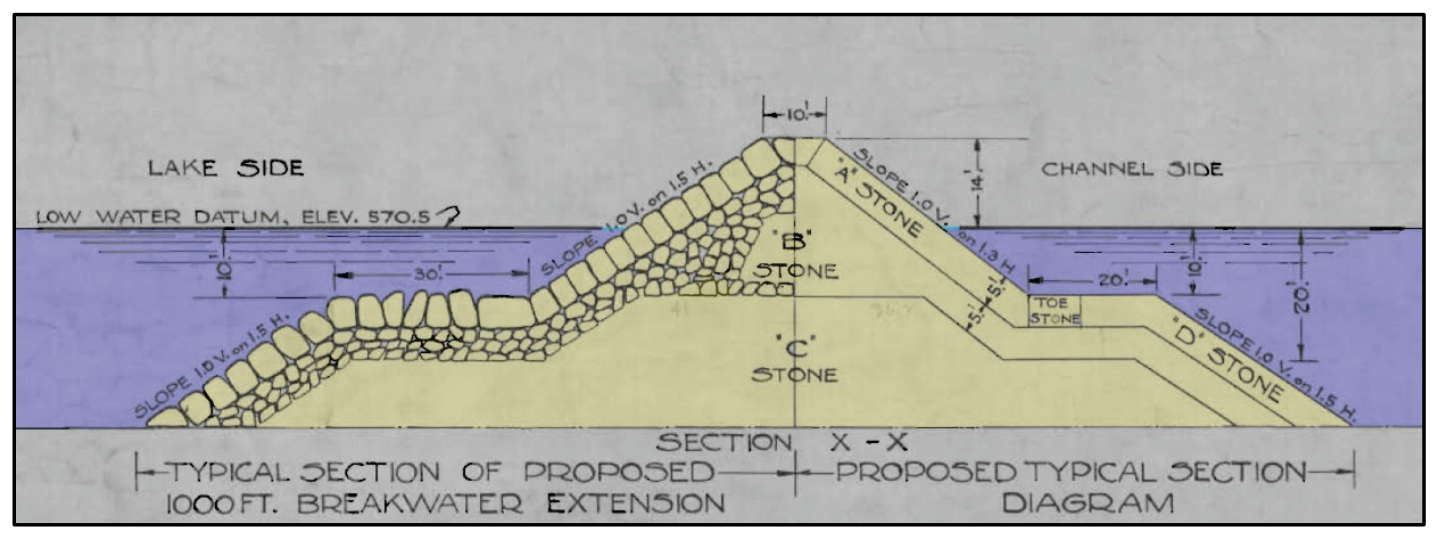


Figure 2-5. SEAB, 12 July 2010. Note extra row of stone placed along crest.

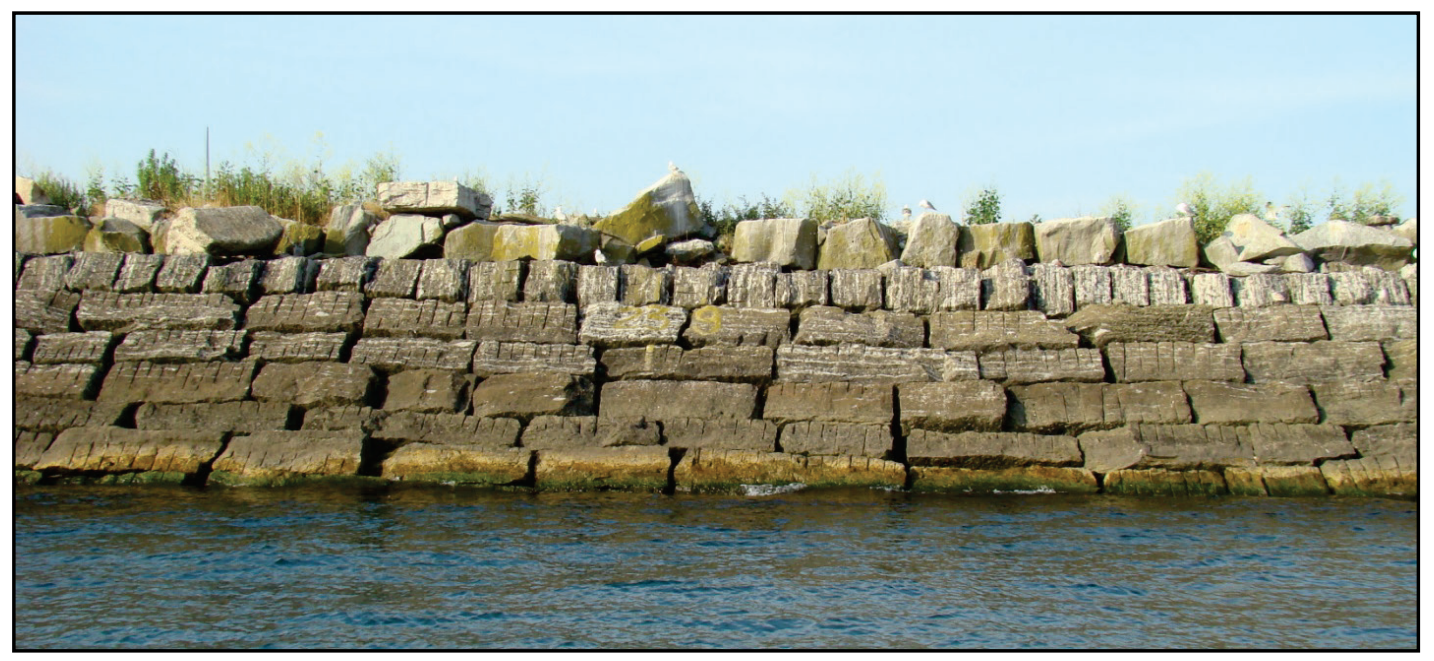

The Perimeter Dike, which completes the encasement of the CDF, was built between August 1974 and June 1977. The $1131.7 \mathrm{~m}$ (3,713 ft) long stone dike was constructed to a crest elevation of $+4.6 \mathrm{~m} \mathrm{LWD} \mathrm{(+15} \mathrm{ft} \mathrm{LWD),} \mathrm{with} \mathrm{a}$

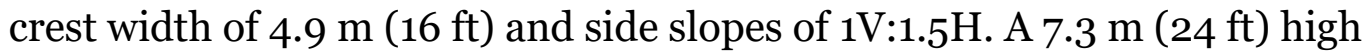
steel sheet pile cut-off wall of $\mathrm{PZ} 27$ piling was constructed along an alignment parallel to and $0.76 \mathrm{~m}(2.5 \mathrm{ft})$ landward of the dike centerline. The cut-off wall extends downward from the top of the dike and projects through the filter stone layer to an elevation of $-2.7 \mathrm{~m}$ LWD (-9.0 ft LWD). Figure 2-6 shows the original typical cross section of the Perimeter Dike.

Figure 2-6. Perimeter Dike typical cross section.

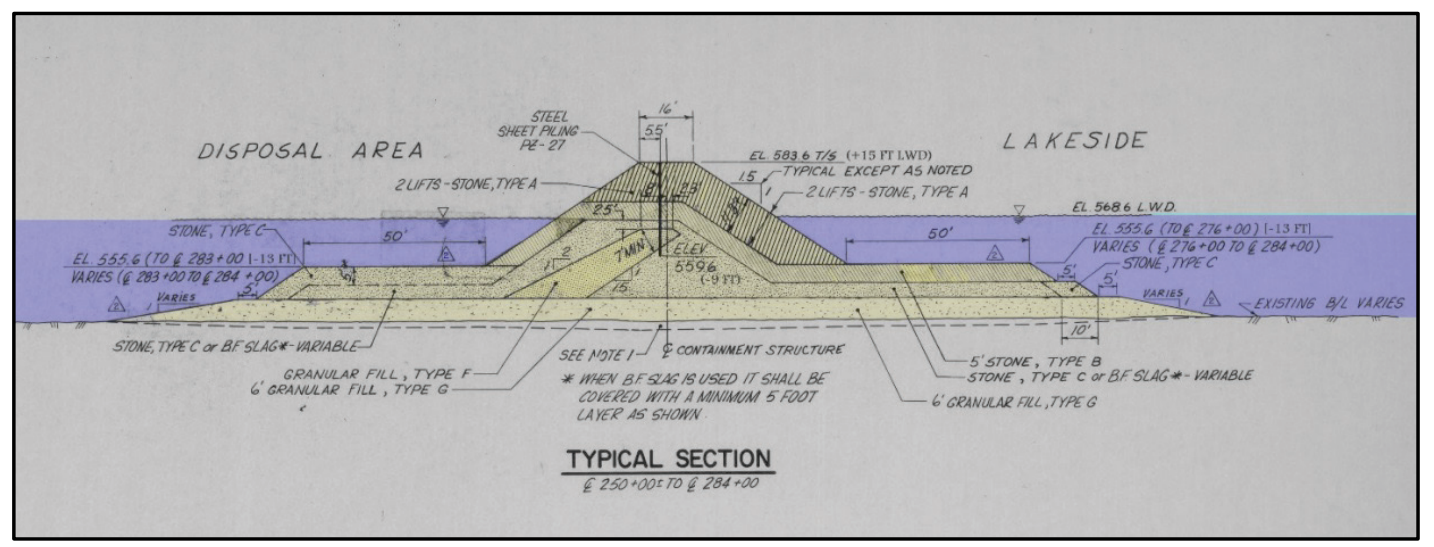

Since its construction, stone quality problems have resulted in stone cracking and stone movement by steepening the side slopes, sporadic lowering of the crest, and movement of the armor stone away from the sheet pile cut-off wall. This latter movement has allowed ice forces to randomly raise the sheet pile upward. The LRB Floating Plant, which is a working/construction platform, has periodically cut the sheets down to the 
original crest elevation, but this raised the concern that it no longer penetrated into the filter layer at those locations. As a result, in July 2000, a contract was let to internally grout 12 reaches (total length $=94.2 \mathrm{~m}$ [309 ft]) by creating a $1.2 \mathrm{~m}$ ( $4 \mathrm{ft}$ ) wide grout curtain overlapping the bottom of the raised sheet pile and extending into the filter blanket.

Recent placement of contaminated sediment dredged from the Buffalo River and the acknowledgement of potential loss of some material from overtopping during significant storm events prompted the creation of an

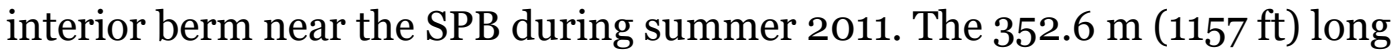
berm, located entirely inside the CDF, is composed of semi-compacted fill taken from borrow areas within the CDF. The crest elevation of the berm is $+4.6 \mathrm{~m} \mathrm{LWD}(+15 \mathrm{ft} \mathrm{LWD})$ and is $9.1 \mathrm{~m}(30 \mathrm{ft})$ wide with side slopes of 1V:4H (Figure 2-7).

Figure 2-7. Interior dike location.

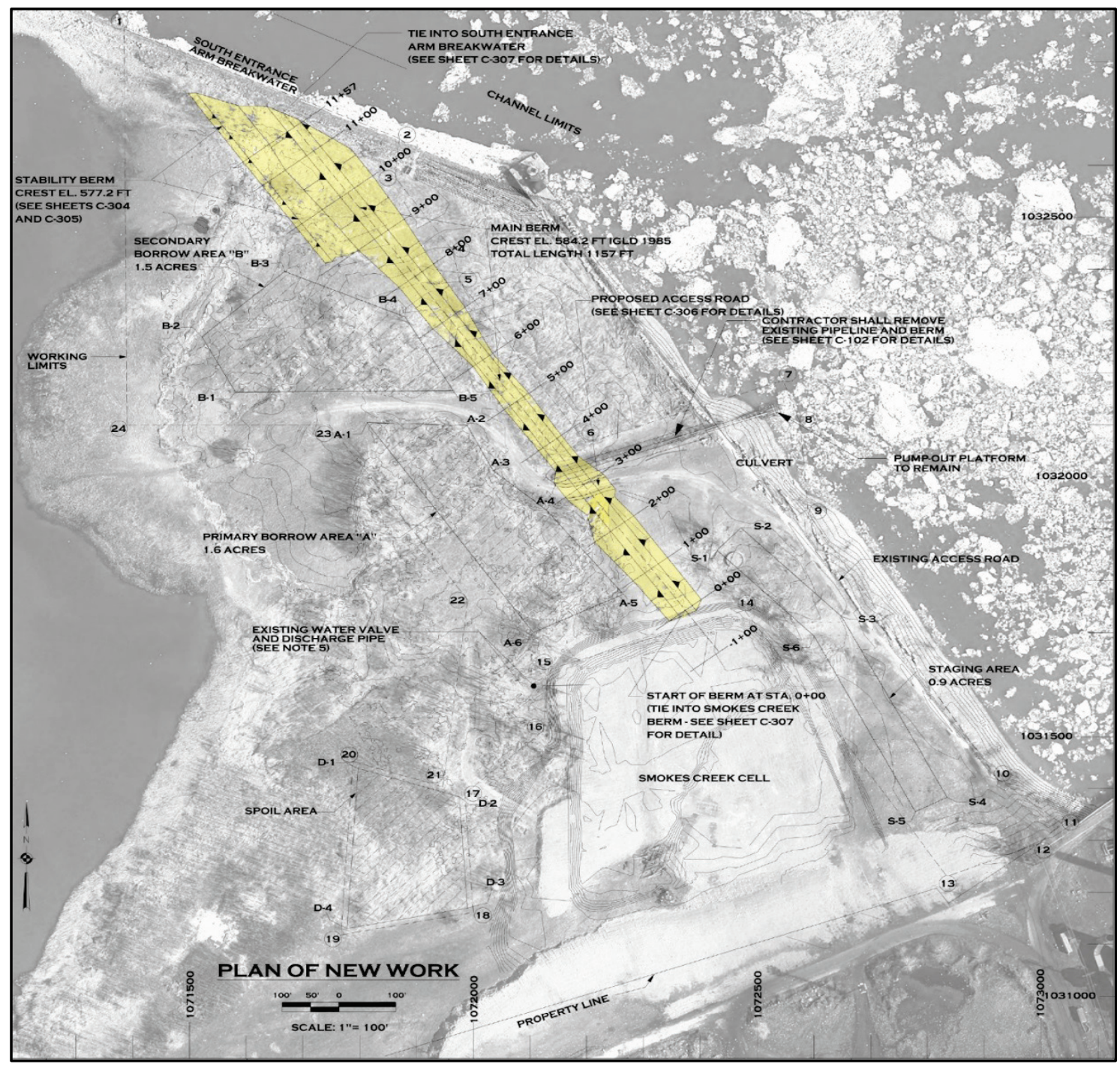


The deterioration, movement, and settlement of the stone along the outer perimeter have resulted in the need to repair the outside and crest of the dike. However, due to limited funds, only a partial repair could be funded. Based upon existing surveys and a stone quality survey completed in summer 2011, the damage along the assessed and repair locations was prioritized.

The project removed the existing crest armor and placed it downslope to fill voids, placing several pieces of underlayer to fill the large voids and then adding chinking stone. Cast-in-place concrete blocks abut the irregular sheet pile cut-off wall along the lakeside crest, restoring the structure to the original crest elevation, and several new armor stones were placed to complete the repair. The repair extends along the majority of Leg 2 and continues along another $115 \mathrm{~m}$ ( $510 \mathrm{ft}$ ) of Leg 3 and was completed in September 2016. Figure 2-8 presents the typical section of the crest repair.

Figure 2-8. Perimeter Dike crest repair cross section.

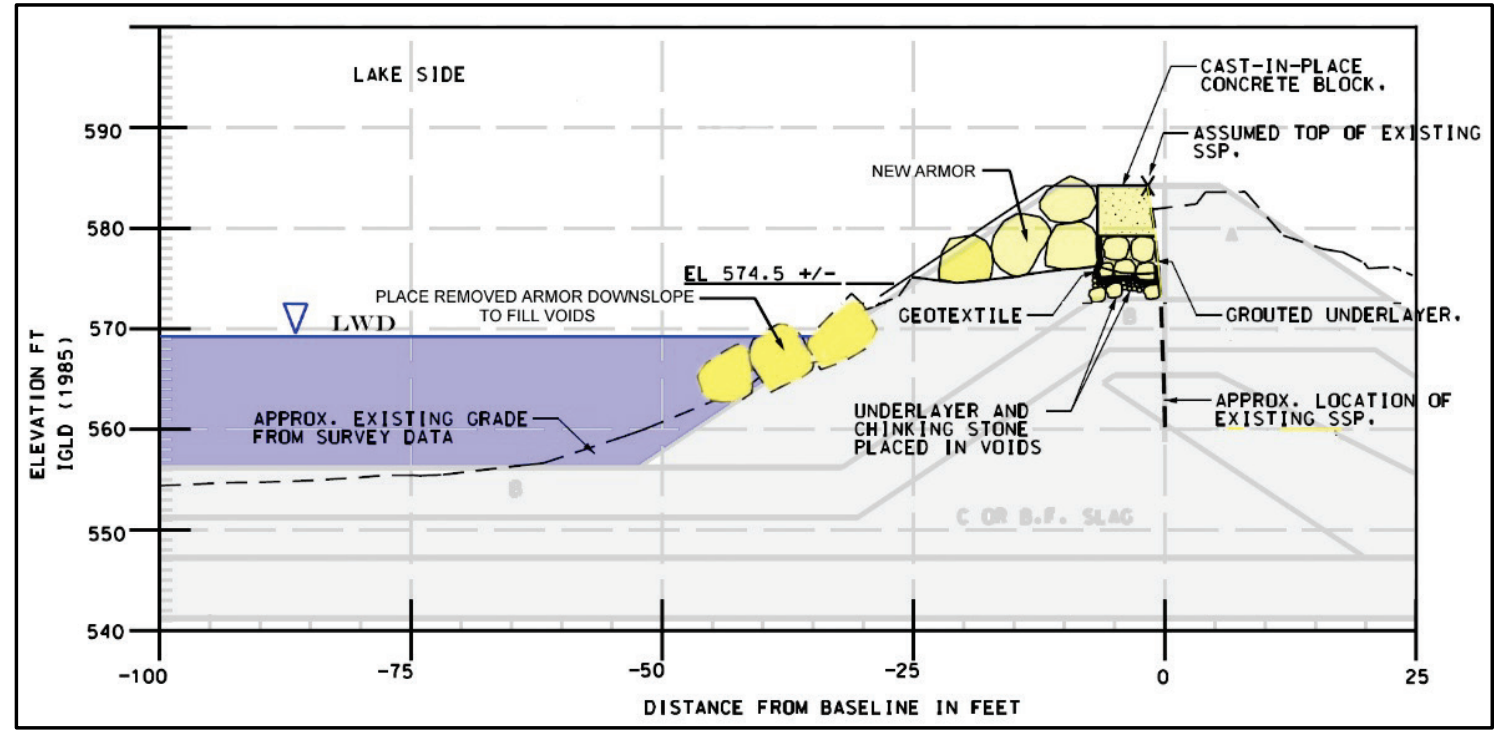

\subsection{Sediment data}

Thirteen surface grab samples (top $0.45 \mathrm{~m}$ [1.5 ft]) were obtained in fall 2015 within $\mathrm{CDF} 4$, and mechanical gradation analyses were performed. The results indicated that the samples were primarily coarse material composed of sand with some gravel/organics, and the fine fraction ranged from approximately $3 \%$ to $61 \%$ with an average silt/clay component of $23.6 \%$. Figure 2-9 presents a map of the location and description of the samples. 
Figure 2-9. Sediment sample locations.

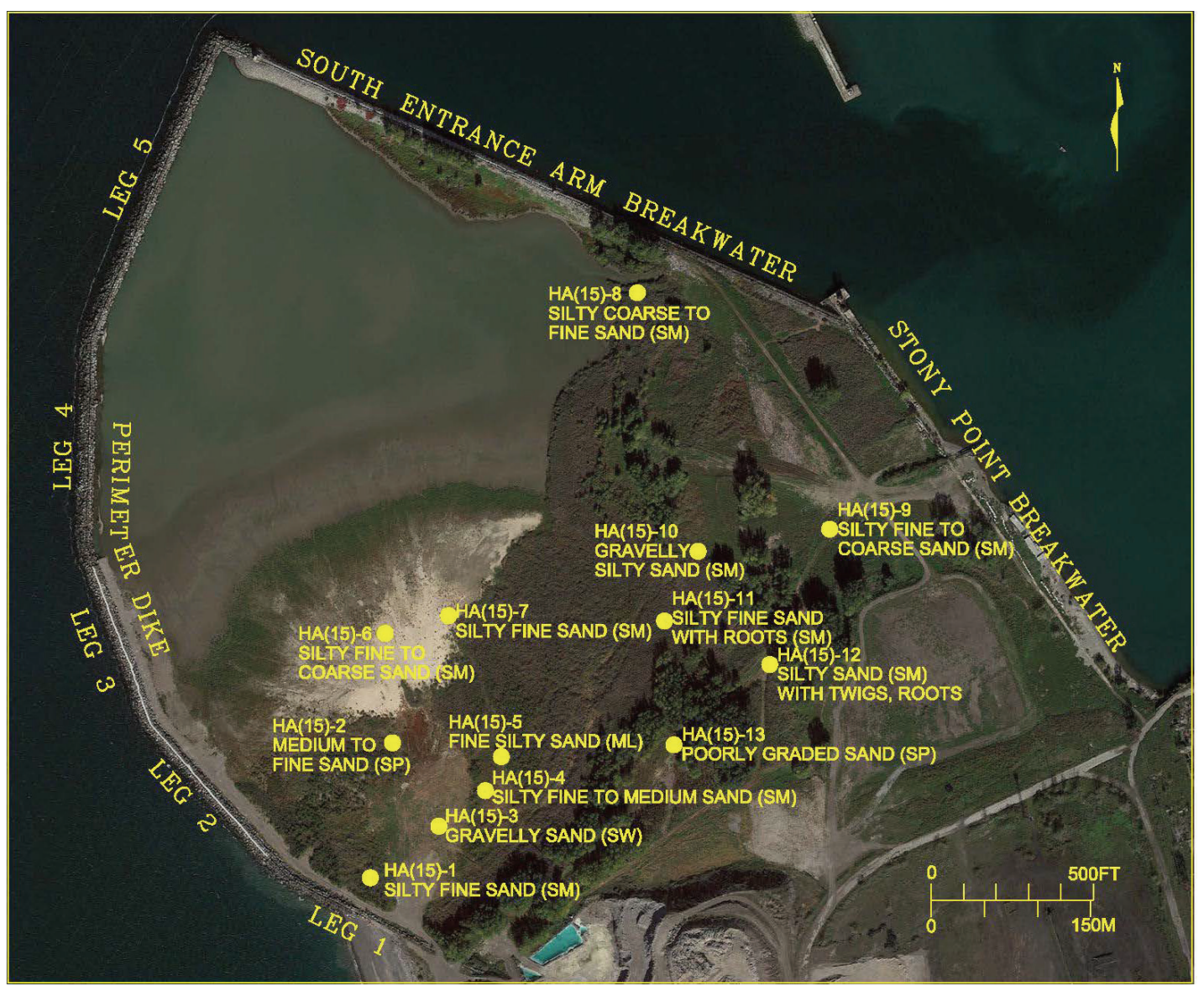

\subsection{Maintenance dredging data}

The need for maintenance dredging arises from sedimentation in the navigation channels, which impedes commercial navigation. Dredging of the Buffalo federal navigation channels from 1996-2015 resulted in the removal of an average of 50,908 cubic yards ( $\left.\mathrm{yd}^{3}\right)(38,922$ cubic meters $\left[\mathrm{m}^{3}\right]$ ) of material annually. Assuming that maintenance dredging of the harbor occurs every other year, approximately $100,000 \mathrm{yd}^{3}\left(76,455 \mathrm{~m}^{3}\right)$ of material would be removed from the harbor during each dredging event (USACE 2016).

In 1987, the Buffalo River was designated as one of 43 "Areas of Concern" (AOC) surrounding the Great Lakes Basin by the International Joint Commission. With this designation, the river was identified as one of the most contaminated waterways in the region due to poor water quality and contaminated sediments. As a result, dredging of the river bottom outside of the federal navigation channel commenced in 2013 , with nearly 1 million yd 3 
$\left(764,555 \mathrm{~m}^{3}\right)$ of contaminated sediment removed from the Buffalo River AOC (Skaros 2015).

It has been determined that there is more than 20 years' capacity for sediment dredged from the harbor in the existing CDF4. This is based upon a conservative, worst-case assumption that confined disposal will be needed for the entire timeframe. Less costly, open-lake placement could, however, become a viable option since the Corps expects to see reduced contaminants in sediment over time. Routine maintenance dredging, strategic navigation dredging, and Great Lakes Legacy Act dredging have removed significant quantities of contaminated sediment from the federal navigation channels, and future sampling and analyses results are expected to reflect improved sediment quality.

\subsection{Future Fill scenarios}

In addition to modeling the existing (2015 survey) fill condition, two future fill scenarios will be modeled. The future conditions include a fill condition less than a decade from present (year 2025) and the dike filled to capacity in year 2036. For the first Future Fill 2025 condition, the fill elevation will be assumed equal to that which presently occurs near the hydraulic dredge pipe outlet $+8 \mathrm{ft}(\sim+2.4 \mathrm{~m})$ LWD and with slopes also mimicking those observed in the 2015 survey. Based upon the additional fill of $477,400 \mathrm{yd}^{3}\left(365,000 \mathrm{~m}^{3}\right)$, approximately $987,500 \mathrm{yd}^{3}\left(755,000 \mathrm{~m}^{3}\right)$ would remain, suggesting this represents the condition at the beginning of FY2O25 (fall 2024). The second Future Fill 2036 condition assumes the CDF will be filled to elevation $+13 \mathrm{ft}$ $(+4 \mathrm{~m})$ LWD by year 2036. For both future conditions, it was assumed that a new berm would be constructed along the south side of the CDF to raise ground elevations to $+15 \mathrm{ft}(+4.6 \mathrm{~m})$ LWD. The future berm scenario location is placed along an existing road to minimize environmental disturbance, but is subject to change.

\subsection{Water level and river discharge data}

A summary of wind, water level, and wave data used in this study is provided in this section. Detailed description of these meteorological and oceanographic (metocean) data and analyses used to develop these data are presented in Appendix C.

Hourly water level data from 1960 to the present time were available from NOAA Coastal Station 9063020 (BUFN6), located on the south side of the 
Buffalo River (http://tidesandcurrents.noaa.gov/) near the upriver end of the U.S. Coast Guard base $\left(42^{\circ} 52.6^{\prime} \mathrm{N}, 78^{\circ} 53.4^{\prime} \mathrm{W}\right)$. Daily discharge data for the Niagara River at Buffalo were available from U.S. Geological Survey (USGS) Station $04216000\left(42^{\circ} 5^{2.67} \mathrm{~N}, 7^{\circ} 54.98^{\prime} \mathrm{W}\right.$ ) from 1926 to the present time (https://waterdata.usgs.gov/nwis/rt). The average annual discharge is approximately 5,800 cubic meters per second ( $\mathrm{m} 3 / \mathrm{sec}$ ) (204,000 cubic feet per second $[\mathrm{ft} 3 / \mathrm{sec}])$. Table 2-1 presents the mean monthly and annual discharges. The Buffalo River discharge can be estimated from three major tributaries: Buffalo Creek, Cayuga Creek, and Cazenovia Creek from USGS-collected river stream data (Irvine et al. 1991). The annual discharge is approximately $20 \mathrm{~m}^{3} / \mathrm{sec}\left(700 \mathrm{ft}^{3} / \mathrm{sec}\right)$. Table 2-2 presents the annual mean discharges for Buffalo River and three major tributaries. Figure 2-10 shows the location map of NOAA 9063020 (BUFN6) and USGS 4216000 stations.

Table 2-1. Mean monthly and annual discharge for the Niagara River at Buffalo, NY.

\begin{tabular}{|l|c|}
\hline Month/Annual & Mean Discharge $\left(\mathrm{ft}^{3} / \mathrm{sec}\right)$ \\
\hline January & 196,000 \\
\hline February & 193,000 \\
\hline March & 199,000 \\
\hline April & 207,000 \\
\hline May & 216,000 \\
\hline June & 215,000 \\
\hline July & 211,000 \\
\hline August & 207,000 \\
\hline September & 203,000 \\
\hline October & 199,000 \\
\hline November & 200,000 \\
\hline December & 201,000 \\
\hline Annual & 204,000 \\
\hline
\end{tabular}

* based on 1926-2015 data from NOAA Coastal Station 9063020 
Table 2-2. Buffalo River average annual discharge.

\begin{tabular}{|l|l|c|c|}
\hline Location & Period of Record & $\begin{array}{c}\text { Drainage } \\
\text { Area SQ } \\
\text { MI }\end{array}$ & $\begin{array}{c}\text { Average Annual } \\
\text { Discharge - } \\
\text { CFS }\end{array}$ \\
\hline $\begin{array}{l}\text { Buffalo Creek at } \\
\text { Gardenville, NY }\end{array}$ & October 1938 to current year & 142 & 207.2 \\
\hline $\begin{array}{l}\text { Cayuga Creek near } \\
\text { Lancaster, NY }\end{array}$ & $\begin{array}{l}\text { September 1938 to } \\
\text { September 1968, annual } \\
\text { maximum only--1972-74, } \\
\text { May 1974 to current year }\end{array}$ & 96.4 & 139.6 \\
\hline $\begin{array}{l}\text { Cazenovia Creek at } \\
\text { Ebenezer, NY }\end{array}$ & June 1940 to current year & 135 & 242.1 \\
\hline Total above & & 373.4 & 588.9 \\
\hline Buffalo River at mouth & & 446 & $703.4 *$ \\
\hline
\end{tabular}

* pro-rated by drainage area

\subsection{Wind and wave data}

Coastal wind and wave data in the study area are available from various sources. NOAA Station 9063020 provides the hourly wind data since 1960. Offshore wave data are available from Environmental and Climate Change Canada (ECCC) (http://www.meds-sdmm.dfo-mpo.gc.ca/isdm-gdsi/waves-vagues/index-eng.htm) Moored Buoy 45142 (Port Colborne; $42^{\circ} 44.6^{\prime}$ N, $79^{\circ}$ 17.6’ W). The GLCFS archived hourly nowcasts for Lake Erie are available for the period of 2006 to present (http://data.glos.us/glcfs/). The USACE WIS has the long-term wind and wave hindcasts for 1979 to 2014. Figure 2-10 shows the location of Buoy 45142 and WIS stations for the eastern end of Lake Erie. In the present modeling study, WIS Station $92243\left(42^{\circ} 48^{\prime} \mathrm{N}, 78^{\circ} 57.6^{\prime} \mathrm{W}\right)$, which is the closest WIS station to CDF4, was used for the incident wave conditions. 
Figure 2-10. Location of NOAA, USGS, WIS, and ECCC coastal stations.

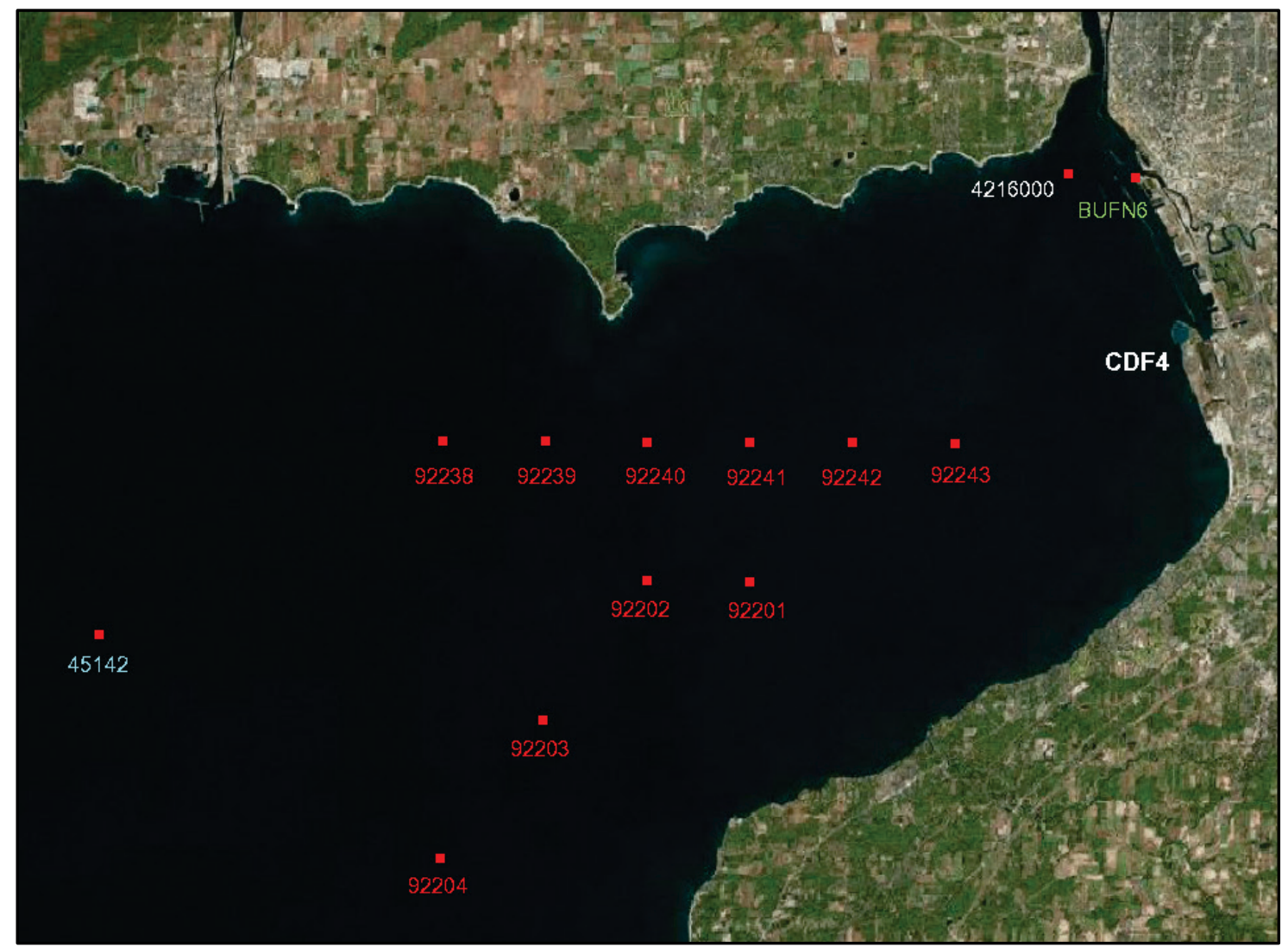

\subsection{Storm data for modeling}

Storm conditions were analyzed based on WIS Station 92243 hindcast data. The analysis identified storms with maximum wave heights greater than $4 \mathrm{~m}$ (13 ft) and mean wave directions within Class Angle 2 encompassing 219 and 279 degrees (deg) azimuth (Figure 2-11). The analysis shows all major

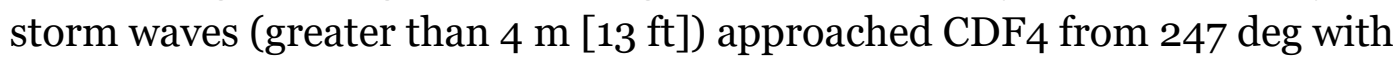
a standard deviation of $1 \mathrm{deg}$. Table 2-3 presents the storm wave events selected from WIS Station 92243. Table 2-4 presents the 2-year, 10-year, 20-year, and 50-year recurrence of storm waves within Class Angle 2 sector at WIS Station 92243. 
Figure 2-11. Class Angle 2 wave directions.

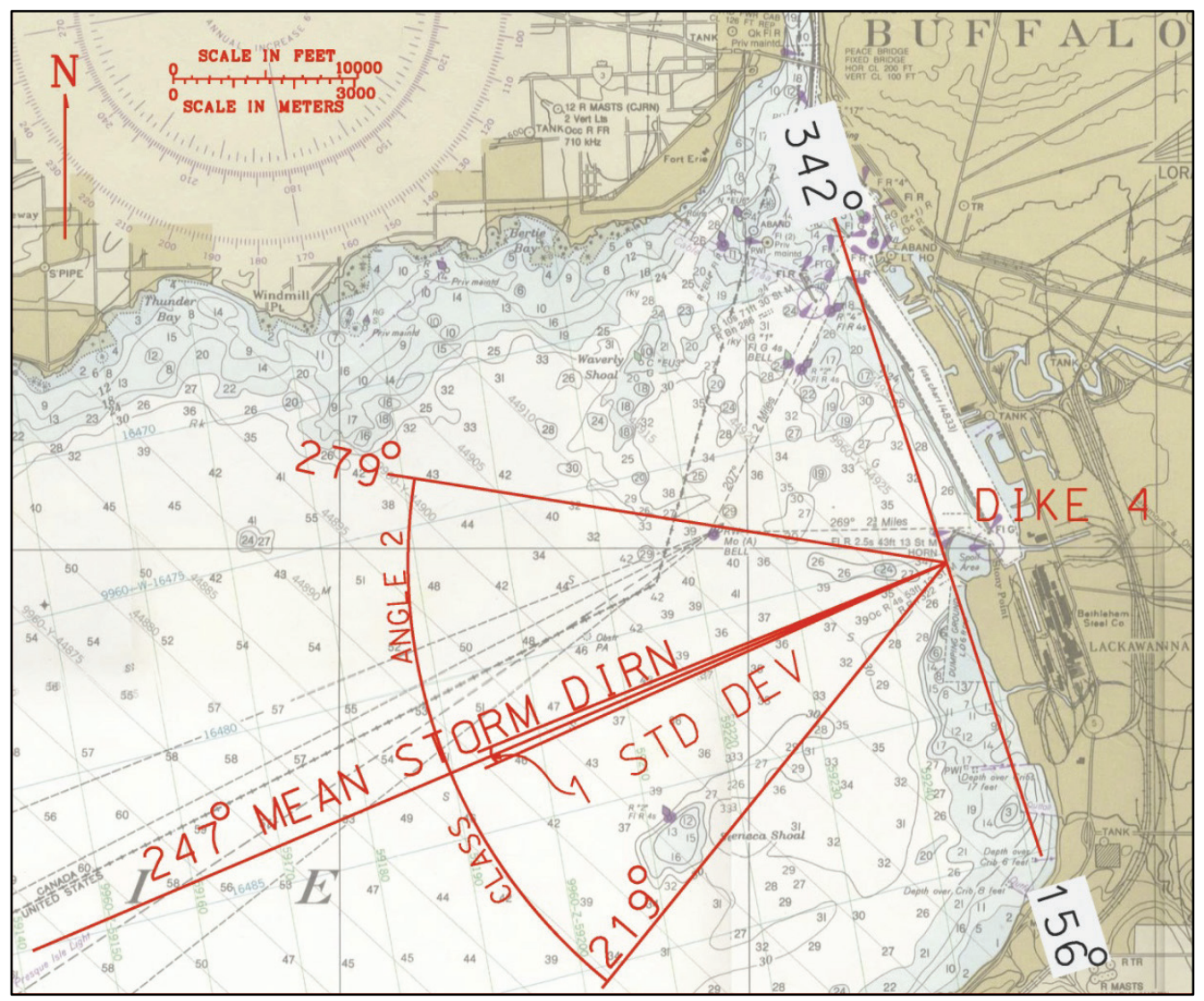

Three storm conditions were selected for wave modeling: (1) 2-year wave and 2-year water level (Storm Condition 1), (2) 20-year wave and 10-year water level (Storm Condition 2), and (3) the actual 11 January 2008 storm (Storm Condition 3) representing a typical average storm wave event in Table 2-3. For Storm Condition 1, the 2-year wave condition was averaged (with storm peak wave height aligned at the same time) from three approximately 2-year recurrence storms: 6 November 1990, 18 December 2000, and 28 April 2011 in Table 2-3. For Storm Condition 2, the 20-year wave condition was averaged from three approximately 20-year recurrence storms: 1 February 2002, 18 January 2012, and 25 December 2013 in Table 2-3. A constant wave direction of 247 deg was used for Storm Conditions 1 and 2. 
Table 2-3. List of selected storm wave events from WIS Station 92243.

\begin{tabular}{|c|c|c|c|c|c|c|}
\hline Rank & $\begin{array}{c}\text { Start } \\
\text { Timestamp* }\end{array}$ & $\begin{array}{c}\text { End } \\
\text { Timestamp* }\end{array}$ & $\begin{array}{c}\text { Peak Wave } \\
\text { Timestamp* }\end{array}$ & $\begin{array}{c}\text { Peak Wave } \\
H_{s}(\mathrm{~m})\end{array}$ & $T p(\sec ) * *$ & $\theta p(\operatorname{deg}) * *$ \\
\hline 1 & 12022419 & 12022607 & 12022501 & 4.85 & 9.7 & 249 \\
\hline 2 & 12011717 & 12011816 & 12011801 & 4.73 & 9.2 & 248 \\
\hline 3 & 02020115 & 02020206 & 02020120 & 4.72 & 10.1 & 247 \\
\hline 4 & 12030304 & 12030418 & 12030313 & 4.68 & 10.0 & 247 \\
\hline 5 & 12012814 & 12013017 & 12012822 & 4.68 & 9.4 & 248 \\
\hline 6 & 06120120 & 06120300 & 06120203 & 4.59 & 10.2 & 247 \\
\hline 7 & 12010116 & 12010305 & 12010203 & 4.57 & 10.0 & 247 \\
\hline 8 & 08122715 & 08122907 & 08122819 & 4.48 & 10.0 & 248 \\
\hline 9 & 11101416 & 11101810 & 11101521 & 4.37 & 10.2 & 248 \\
\hline 10 & 82011015 & 82011206 & 82011112 & 4.37 & 10.2 & 247 \\
\hline 11 & 85120120 & 85120313 & 85120213 & 4.36 & 10.0 & 247 \\
\hline 12 & 03111303 & 03111410 & 03111314 & 4.33 & 10.9 & 247 \\
\hline 13 & 82010412 & 82010521 & 82010503 & 4.30 & 10.8 & 246 \\
\hline 14 & 09120914 & 09121212 & 09121005 & 4.29 & 10.8 & 246 \\
\hline 15 & 87121514 & 87121706 & 87121608 & 4.27 & 11.0 & 246 \\
\hline 16 & 00121715 & 00121820 & 00121803 & 4.22 & 10.0 & 247 \\
\hline 17 & 90110603 & 90110623 & 90110611 & 4.15 & 10.4 & 247 \\
\hline 18 & 11042811 & 11042903 & 11042816 & 4.11 & 9.2 & 246 \\
\hline 19 & 08010905 & 08011005 & 08010913 & 4.11 & 8.5 & 249 \\
\hline 20 & 06020507 & 06020712 & 06020514 & 4.10 & 9.6 & 246 \\
\hline 21 & 89010804 & 89010923 & 89010820 & 4.06 & 9.7 & 248 \\
\hline 22 & 12011308 & 12011411 & 12011315 & 4.01 & 9.2 & 247 \\
\hline
\end{tabular}

* timestamp given in yymmddhh (yy for year, mm for month, dd for date, hh for hour, GMT)

** wave period $T_{p}$ and direction $\theta_{p}$ associated with the peak wave height $H_{S}$

Table 2-4. Class Angle 2 recurrence of storm wave heights and associated wave period.

\begin{tabular}{|c|c|c|}
\hline Return Period (year) & $H_{\boldsymbol{s}}(\mathrm{m})$ & $T p(\mathrm{sec})$ \\
\hline 2 & 4.2 & 9.8 \\
\hline 10 & 4.7 & 10.2 \\
\hline 20 & 4.8 & 10.4 \\
\hline 50 & 5.1 & 10.6 \\
\hline
\end{tabular}


Based on WIS 92243 data, the surface wind speed forcings for Storm Conditions 1 and 2 were determined from a linear relation of wind speed $U_{1 O}$ (at a $10 \mathrm{~m}[33 \mathrm{ft}]$ level above the lake surface) and wave height $H s$ within Angle 2 sector:

$$
\text { Surface wind speed } \mathrm{U}_{10}(\mathrm{~m} / \mathrm{sec})=4.2957 \mathrm{Hs}(\mathrm{m})+2.5385
$$

The correlation coefficient of $U_{1 O}$ and $H_{S}$ data is 0.92. A constant wind direction of $247 \mathrm{deg}$ was used in Storm Conditions 1 and 2. Based on the long-term water level data collected at NOAA Station 9063020 from 1900 to present, the annual maxima were analyzed for the different design recurrence intervals (return periods). Table 2-5 presents the water levels for 2-year, 10-year, 20-year, and 50-year recurrence intervals.

Table 2-5. Water levels at NOAA Station 9063020 for several recurrence intervals.

\begin{tabular}{|c|c|}
\hline Return Period (year) & Water Level (m), IGLD 1985 \\
\hline 2 & 175.80 \\
\hline 10 & 176.45 \\
\hline 20 & 176.56 \\
\hline 50 & 176.74 \\
\hline
\end{tabular}

The 2-year storm water level condition was averaged (with storm water level peak aligned at the same time) from three approximately 2-year recurrence storms: 13 November 2003, 7 October 2009, and 25 December 2014 in Table 2-3. Similarly, the 10-year storm water level condition was averaged from three approximately 10-year recurrence storms: 12 December 2000, 10 March 2002, and 1 December 2006 in Table 2-3. For Storm Conditions 1 and 2 , the peak of the waves was aligned with the peak of the water level.

Tables 2-6 to 2-8 present the hourly wave height, wave period, wave direction, wind speed, wind direction, and water level for Storm Conditions 1, 2, and 3: (1) 2-year wave and 2-year water level, (2) 20-year wave and 10-year water level, and (3) the actual 11 January 2008 storm event, respectively. Storm Conditions 1 and 2 includes a 50-hour (hr) period while Storm Condition 3, with wave height greater than $1 \mathrm{~m}$ (3.3 ft), covers a $40 \mathrm{hr}$ duration. 
Table 2-6. Hourly data for the 2-year wave and 2-year water level storm (Storm Condition 1).

\begin{tabular}{|c|c|c|c|c|c|c|}
\hline Time (hr) & $H_{s}(\mathrm{~m})$ & $T p$ (sec) & $\theta_{p}(\mathrm{deg})$ & $\begin{array}{l}U_{10} \\
(\mathrm{sec})\end{array}$ & $\begin{array}{l}\text { Wind } \\
\text { Direction } \\
\text { (deg) }\end{array}$ & $\begin{array}{l}\text { Water Level (m) } \\
\text { IGLD } 1985\end{array}$ \\
\hline 0 & 0.55 & 3.73 & 247 & 4.4 & 247 & 174.02 \\
\hline 1 & 0.57 & 3.82 & 247 & 4.4 & 247 & 174.03 \\
\hline 2 & 0.64 & 4.01 & 247 & 4.8 & 247 & 174.06 \\
\hline 3 & 0.86 & 4.44 & 247 & 5.7 & 247 & 174.05 \\
\hline 4 & 1.10 & 4.99 & 247 & 6.8 & 247 & 174.02 \\
\hline 5 & 1.34 & 5.58 & 247 & 7.8 & 247 & 174.06 \\
\hline 6 & 1.61 & 6.16 & 247 & 8.9 & 247 & 174.13 \\
\hline 7 & 1.78 & 6.70 & 247 & 9.6 & 247 & 174.19 \\
\hline 8 & 2.04 & 6.15 & 247 & 10.6 & 247 & 174.29 \\
\hline 9 & 2.37 & 6.81 & 247 & 11.9 & 247 & 174.47 \\
\hline 10 & 2.73 & 7.43 & 247 & 13.3 & 247 & 174.54 \\
\hline 11 & 3.17 & 8.02 & 247 & 14.9 & 247 & 174.73 \\
\hline 12 & 3.63 & 8.74 & 247 & 16.5 & 247 & 175.07 \\
\hline 13 & 4.06 & 9.36 & 247 & 17.9 & 247 & 175.44 \\
\hline 14 & 4.20 & 9.80 & 247 & 18.4 & 247 & 175.80 \\
\hline 15 & 4.14 & 9.96 & 247 & 18.2 & 247 & 175.63 \\
\hline 16 & 3.87 & 9.93 & 247 & 17.3 & 247 & 175.55 \\
\hline 17 & 3.45 & 9.77 & 247 & 15.9 & 247 & 175.31 \\
\hline 18 & 3.15 & 9.32 & 247 & 14.8 & 247 & 175.23 \\
\hline 19 & 2.99 & 9.04 & 247 & 14.2 & 247 & 174.95 \\
\hline 20 & 2.76 & 8.52 & 247 & 13.4 & 247 & 174.81 \\
\hline 21 & 2.44 & 8.27 & 247 & 12.2 & 247 & 174.71 \\
\hline 22 & 2.15 & 7.77 & 247 & 11.1 & 247 & 174.65 \\
\hline 23 & 1.88 & 7.49 & 247 & 10.0 & 247 & 174.56 \\
\hline 24 & 1.69 & 7.09 & 247 & 9.2 & 247 & 174.38 \\
\hline 25 & 1.54 & 6.82 & 247 & 8.6 & 247 & 174.43 \\
\hline 26 & 1.38 & 6.71 & 247 & 7.9 & 247 & 174.52 \\
\hline 27 & 1.23 & 6.32 & 247 & 7.3 & 247 & 174.42 \\
\hline 28 & 1.08 & 6.21 & 247 & 6.7 & 247 & 174.37 \\
\hline 29 & 0.98 & 6.26 & 247 & 6.2 & 247 & 174.40 \\
\hline 30 & 0.93 & 5.49 & 247 & 6.0 & 247 & 174.42 \\
\hline 31 & 0.85 & 5.48 & 247 & 5.7 & 247 & 174.38 \\
\hline 32 & 0.78 & 5.25 & 247 & 5.4 & 247 & 174.36 \\
\hline 33 & 0.72 & 5.25 & 247 & 5.1 & 247 & 174.40 \\
\hline 34 & 0.70 & 5.09 & 247 & 5.0 & 247 & 174.36 \\
\hline 35 & 0.71 & 5.11 & 247 & 5.1 & 247 & 174.28 \\
\hline 36 & 0.70 & 5.23 & 247 & 5.0 & 247 & 174.20 \\
\hline
\end{tabular}




\begin{tabular}{|l|l|l|l|l|l|l|}
\hline Time $(\mathrm{hr})$ & $H_{\boldsymbol{S}}(\mathrm{m})$ & $T p(\mathbf{s e c})$ & $\boldsymbol{\theta}_{p}(\mathrm{deg})$ & $\begin{array}{l}U_{10} \\
(\mathbf{s e c})\end{array}$ & $\begin{array}{l}\text { Wind } \\
\text { Direction } \\
(\mathrm{deg})\end{array}$ & $\begin{array}{l}\text { Water Level }(\mathrm{m}) \\
\text { IGLD 1985 }\end{array}$ \\
\hline 37 & 0.65 & 5.26 & 247 & 4.8 & 247 & 174.15 \\
\hline 38 & 0.61 & 5.27 & 247 & 4.6 & 247 & 174.11 \\
\hline 39 & 0.57 & 5.19 & 247 & 4.4 & 247 & 174.09 \\
\hline 40 & 0.54 & 5.05 & 247 & 4.3 & 247 & 174.10 \\
\hline 41 & 0.52 & 4.97 & 247 & 4.2 & 247 & 174.13 \\
\hline 42 & 0.50 & 4.94 & 247 & 4.1 & 247 & 174.21 \\
\hline 43 & 0.47 & 4.88 & 247 & 4.0 & 247 & 174.23 \\
\hline 44 & 0.45 & 4.22 & 247 & 3.9 & 247 & 174.30 \\
\hline 45 & 0.43 & 4.33 & 247 & 3.8 & 247 & 174.32 \\
\hline 46 & 0.42 & 4.47 & 247 & 3.8 & 247 & 174.27 \\
\hline 47 & 0.42 & 4.52 & 247 & 3.8 & 247 & 174.26 \\
\hline 48 & 0.45 & 3.90 & 247 & 3.9 & 247 & 174.33 \\
\hline 49 & 0.47 & 4.10 & 247 & 4.0 & 247 & 174.31 \\
\hline 50 & 0.50 & 4.29 & 247 & 4.1 & 247 & 174.19 \\
\hline
\end{tabular}

Table 2-7. Hourly data for the 20-year wave and 10-year water level storm (Storm Condition 2).

\begin{tabular}{|c|c|c|c|c|c|c|}
\hline Time $(\mathrm{hr})$ & $H_{\boldsymbol{s}}(\mathrm{m})$ & $T p(\mathbf{s e c})$ & $\boldsymbol{\theta}_{\boldsymbol{p}(\mathrm{deg})}$ & $\begin{array}{c}\boldsymbol{U}_{10} \\
(\mathbf{s e c})\end{array}$ & $\begin{array}{c}\text { Wind } \\
\text { Direction } \\
(\mathrm{deg})\end{array}$ & $\begin{array}{c}\text { Water Level (m) } \\
\text { IGLD 1985 }\end{array}$ \\
\hline 0 & 0.34 & 2.94 & 247 & 3.4 & 247 & 173.84 \\
\hline 1 & 0.34 & 3.10 & 247 & 3.4 & 247 & 173.77 \\
\hline 2 & 0.34 & 3.13 & 247 & 3.4 & 247 & 173.77 \\
\hline 3 & 0.34 & 3.17 & 247 & 3.4 & 247 & 173.75 \\
\hline 4 & 0.36 & 3.23 & 247 & 3.5 & 247 & 173.77 \\
\hline 5 & 0.40 & 2.91 & 247 & 3.7 & 247 & 173.77 \\
\hline 6 & 0.50 & 3.21 & 247 & 4.1 & 247 & 173.69 \\
\hline 7 & 0.62 & 3.43 & 247 & 4.7 & 247 & 173.76 \\
\hline 8 & 0.79 & 3.89 & 247 & 5.4 & 247 & 173.83 \\
\hline 9 & 1.36 & 5.12 & 247 & 7.9 & 247 & 173.85 \\
\hline 10 & 2.16 & 6.20 & 247 & 11.1 & 247 & 174.29 \\
\hline 11 & 2.95 & 7.22 & 247 & 14.1 & 247 & 174.72 \\
\hline 12 & 3.77 & 8.48 & 247 & 17.0 & 247 & 174.83 \\
\hline 13 & 4.45 & 9.63 & 247 & 19.2 & 247 & 175.51 \\
\hline 14 & 4.80 & 10.40 & 247 & 20.2 & 247 & 176.45 \\
\hline 15 & 4.54 & 10.63 & 247 & 19.4 & 247 & 176.27 \\
\hline 16 & 3.96 & 10.45 & 247 & 17.6 & 247 & 175.58 \\
\hline 17 & 3.50 & 10.10 & 247 & 16.0 & 247 & 175.25 \\
\hline 18 & 3.17 & 9.72 & 247 & 14.9 & 247 & 175.29 \\
\hline & & & & & & \\
\hline
\end{tabular}




\begin{tabular}{|c|c|c|c|c|c|c|}
\hline Time (hr) & $H_{s}(\mathrm{~m})$ & $T p(\mathrm{sec})$ & $\theta_{p}(\mathrm{deg})$ & $\begin{array}{l}U_{10} \\
(\mathrm{sec})\end{array}$ & $\begin{array}{l}\text { Wind } \\
\text { Direction } \\
\text { (deg) }\end{array}$ & $\begin{array}{l}\text { Water Level (m) } \\
\text { IGLD } 1985\end{array}$ \\
\hline 19 & 2.91 & 9.30 & 247 & 13.9 & 247 & 174.99 \\
\hline 20 & 2.77 & 9.06 & 247 & 13.4 & 247 & 174.74 \\
\hline 21 & 2.63 & 8.73 & 247 & 12.9 & 247 & 174.58 \\
\hline 22 & 2.41 & 8.51 & 247 & 12.1 & 247 & 174.65 \\
\hline 23 & 2.16 & 8.16 & 247 & 11.1 & 247 & 174.46 \\
\hline 24 & 1.94 & 7.94 & 247 & 10.2 & 247 & 174.19 \\
\hline 25 & 1.74 & 7.66 & 247 & 9.4 & 247 & 174.33 \\
\hline 26 & 1.61 & 7.47 & 247 & 8.9 & 247 & 174.61 \\
\hline 27 & 1.54 & 7.33 & 247 & 8.6 & 247 & 174.70 \\
\hline 28 & 1.47 & 7.23 & 247 & 8.3 & 247 & 174.49 \\
\hline 29 & 1.37 & 7.22 & 247 & 7.9 & 247 & 174.44 \\
\hline 30 & 1.24 & 7.23 & 247 & 7.4 & 247 & 174.56 \\
\hline 31 & 1.12 & 6.84 & 247 & 6.8 & 247 & 174.52 \\
\hline 32 & 1.03 & 6.75 & 247 & 6.5 & 247 & 174.43 \\
\hline 33 & 0.97 & 6.83 & 247 & 6.2 & 247 & 174.37 \\
\hline 34 & 0.92 & 6.68 & 247 & 6.0 & 247 & 174.33 \\
\hline 35 & 0.87 & 6.62 & 247 & 5.8 & 247 & 174.20 \\
\hline 36 & 0.83 & 6.45 & 247 & 5.6 & 247 & 174.04 \\
\hline 37 & 0.80 & 6.11 & 247 & 5.5 & 247 & 173.99 \\
\hline 38 & 0.77 & 6.00 & 247 & 5.3 & 247 & 174.00 \\
\hline 39 & 0.72 & 6.04 & 247 & 5.1 & 247 & 174.04 \\
\hline 40 & 0.69 & 5.97 & 247 & 5.0 & 247 & 174.01 \\
\hline 41 & 0.66 & 4.86 & 247 & 4.8 & 247 & 174.03 \\
\hline 42 & 0.65 & 4.81 & 247 & 4.8 & 247 & 174.08 \\
\hline 43 & 0.64 & 4.79 & 247 & 4.8 & 247 & 174.16 \\
\hline 44 & 0.62 & 4.75 & 247 & 4.7 & 247 & 174.24 \\
\hline 45 & 0.60 & 4.70 & 247 & 4.6 & 247 & 174.18 \\
\hline 46 & 0.61 & 4.60 & 247 & 4.6 & 247 & 174.17 \\
\hline 47 & 0.62 & 4.66 & 247 & 4.7 & 247 & 174.14 \\
\hline 48 & 0.65 & 4.80 & 247 & 4.8 & 247 & 174.12 \\
\hline 49 & 0.71 & 5.00 & 247 & 5.1 & 247 & 174.07 \\
\hline 50 & 0.80 & 5.01 & 247 & 5.5 & 247 & 174.01 \\
\hline
\end{tabular}


Table 2-8. Hourly data for the 11 January 1982 storm (Storm Condition 3).

\begin{tabular}{|c|c|c|c|c|c|c|}
\hline Timestamp & $H_{s}(\mathrm{~m})$ & $T p(\mathrm{sec})$ & $\theta_{p}(\mathrm{deg})$ & $U_{10}(\mathrm{sec})$ & $\begin{array}{l}\text { Wind } \\
\text { Direction } \\
\text { (deg) }\end{array}$ & $\begin{array}{l}\text { Water Level (m) } \\
\text { IGLD } 1985\end{array}$ \\
\hline 82011015 & 1.06 & 3.90 & 260 & 11.6 & 286 & 174.637 \\
\hline 82011016 & 1.47 & 4.94 & 260 & 12.4 & 276 & 174.917 \\
\hline 82011017 & 1.93 & 5.82 & 257 & 13.4 & 270 & 175.119 \\
\hline 82011018 & 2.52 & 6.66 & 254 & 15.2 & 262 & 175.116 \\
\hline 82011019 & 2.97 & 7.46 & 252 & 15.8 & 261 & 175.027 \\
\hline 82011020 & 3.19 & 8.24 & 252 & 15.8 & 260 & 175.609 \\
\hline 82011021 & 3.24 & 8.82 & 251 & 15.4 & 258 & 175.981 \\
\hline 82011022 & 3.34 & 9.15 & 249 & 15.9 & 253 & 176.149 \\
\hline 82011023 & 3.56 & 9.59 & 249 & 16.7 & 249 & 176.191 \\
\hline 82011100 & 3.86 & 9.98 & 247 & 19.8 & 233 & 176.362 \\
\hline 82011101 & 4.28 & 10.20 & 248 & 20.3 & 243 & 176.176 \\
\hline 82011102 & 4.34 & 10.46 & 248 & 19.2 & 244 & 176.048 \\
\hline 82011103 & 4.12 & 10.67 & 248 & 18.4 & 243 & 175.844 \\
\hline 82011104 & 3.93 & 10.65 & 247 & 17.5 & 242 & 175.804 \\
\hline 82011105 & 3.69 & 10.44 & 247 & 16.5 & 241 & 175.652 \\
\hline 82011106 & 3.47 & 10.20 & 247 & 15.6 & 243 & 175.567 \\
\hline 82011107 & 3.35 & 10.00 & 247 & 16.1 & 242 & 175.466 \\
\hline 82011108 & 3.37 & 9.84 & 247 & 16.7 & 241 & 175.582 \\
\hline 82011109 & 3.52 & 9.76 & 248 & 17.8 & 240 & 175.707 \\
\hline 82011110 & 3.82 & 9.80 & 248 & 19.4 & 240 & 175.801 \\
\hline 82011111 & 4.20 & 10.00 & 248 & 20.4 & 240 & 175.820 \\
\hline 82011112 & 4.37 & 10.17 & 247 & 21.2 & 229 & 175.978 \\
\hline 82011113 & 4.25 & 10.30 & 247 & 18.7 & 245 & 176.015 \\
\hline 82011114 & 3.70 & 10.30 & 248 & 14.6 & 263 & 175.844 \\
\hline 82011115 & 3.03 & 10.12 & 248 & 12.0 & 284 & 175.719 \\
\hline 82011116 & 2.63 & 9.98 & 248 & 12.2 & 282 & 175.579 \\
\hline 82011117 & 2.59 & 9.92 & 247 & 12.5 & 279 & 175.399 \\
\hline 82011118 & 2.77 & 9.86 & 248 & 13.4 & 275 & 175.557 \\
\hline 82011119 & 2.91 & 9.86 & 248 & 13.5 & 278 & 175.469 \\
\hline 82011120 & 2.85 & 9.86 & 248 & 12.3 & 282 & 175.271 \\
\hline 82011121 & 2.63 & 9.80 & 248 & 11.0 & 284 & 175.213 \\
\hline
\end{tabular}




\begin{tabular}{|c|c|c|c|c|c|c|}
\hline Timestamp & $H_{\boldsymbol{S}}(\mathrm{m})$ & $T p(\mathrm{sec})$ & $\boldsymbol{\theta} p(\mathrm{deg})$ & $U_{10}(\mathrm{sec})$ & $\begin{array}{c}\text { Wind } \\
\text { Direction } \\
(\mathrm{deg})\end{array}$ & $\begin{array}{c}\text { Water Level }(\mathrm{m}) \\
\text { IGLD 1985 }\end{array}$ \\
\hline 82011122 & 2.38 & 9.66 & 248 & 9.8 & 290 & 175.158 \\
\hline 82011123 & 2.15 & 9.34 & 247 & 8.7 & 297 & 174.911 \\
\hline 82011200 & 1.97 & 9.16 & 247 & 7.9 & 297 & 174.814 \\
\hline 82011201 & 1.81 & 9.09 & 247 & 7.1 & 316 & 174.735 \\
\hline 82011202 & 1.66 & 9.04 & 246 & 7.1 & 334 & 174.597 \\
\hline 82011203 & 1.51 & 8.96 & 246 & 6.7 & 349 & 174.515 \\
\hline 82011204 & 1.35 & 8.70 & 246 & 5.9 & 355 & 174.515 \\
\hline 82011205 & 1.21 & 8.38 & 246 & 5.5 & 3 & 174.478 \\
\hline 82011206 & 1.09 & 8.28 & 246 & 4.9 & 3 & 174.411 \\
\hline
\end{tabular}

Storm Condition 1 has a maximum wave height of $4.2 \mathrm{~m}(13.8 \mathrm{ft}$ ) at Hour 14, coincident with the highest water level of $175.80 \mathrm{~m}$ (IGLD, 1985) or $2.3 \mathrm{~m}$ above LWD. Storm Condition 2 has a maximum wave height of $4.8 \mathrm{~m}$ at Hour 14, coincident with the highest water level of $176.45 \mathrm{~m}$ (IGLD, 1985) or $3.0 \mathrm{~m}$ above the LWD. A constant wind and wave direction of $247 \mathrm{deg}$ was assumed in Storm Conditions 1 and 2. In Storm Condition 3, the maximum wave height of $4.37 \mathrm{~m}$ occurred at 12:00 GMT, 11 January 1982, while the highest water level of $176.36 \mathrm{~m}$ or $2.9 \mathrm{~m}$ above the LWD occurred approximately $12 \mathrm{hr}$ prior to the maximum wave height. Note that the wave height corresponding to the highest water level in Storm Condition 3 is 4.34 $\mathrm{m}$, which is just slightly less than the maximum wave height. Figures 2-12 to 2-14 show the time series of wave height, wave period, wind speed, and water level for Storm Conditions 1, 2, and 3, respectively. 
Figure 2-12. The 2-year wave and 2-year water level storm (Storm Condition 1).

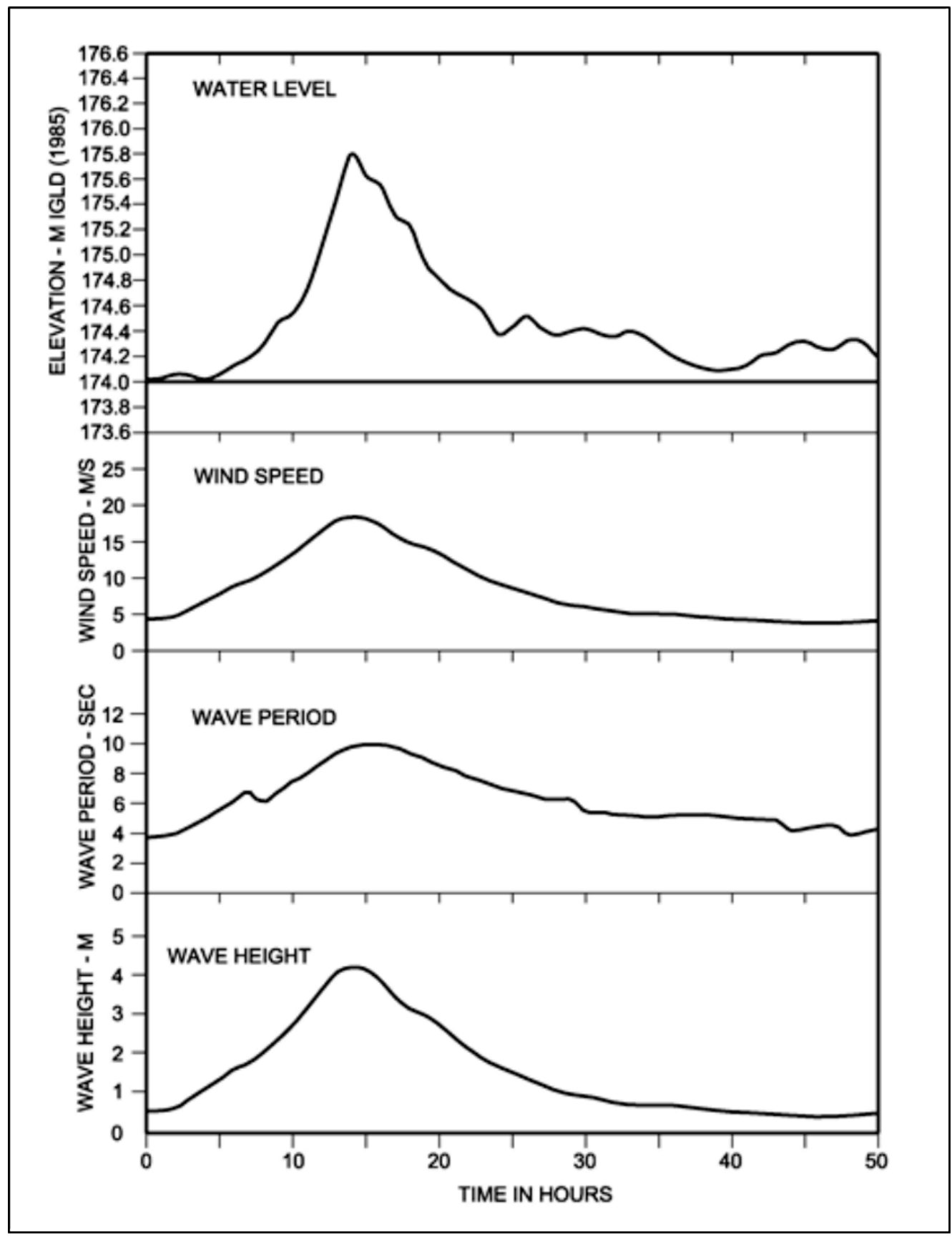


Figure 2-13. The 20-year wave and 10-year water level storm (Storm Condition 2).

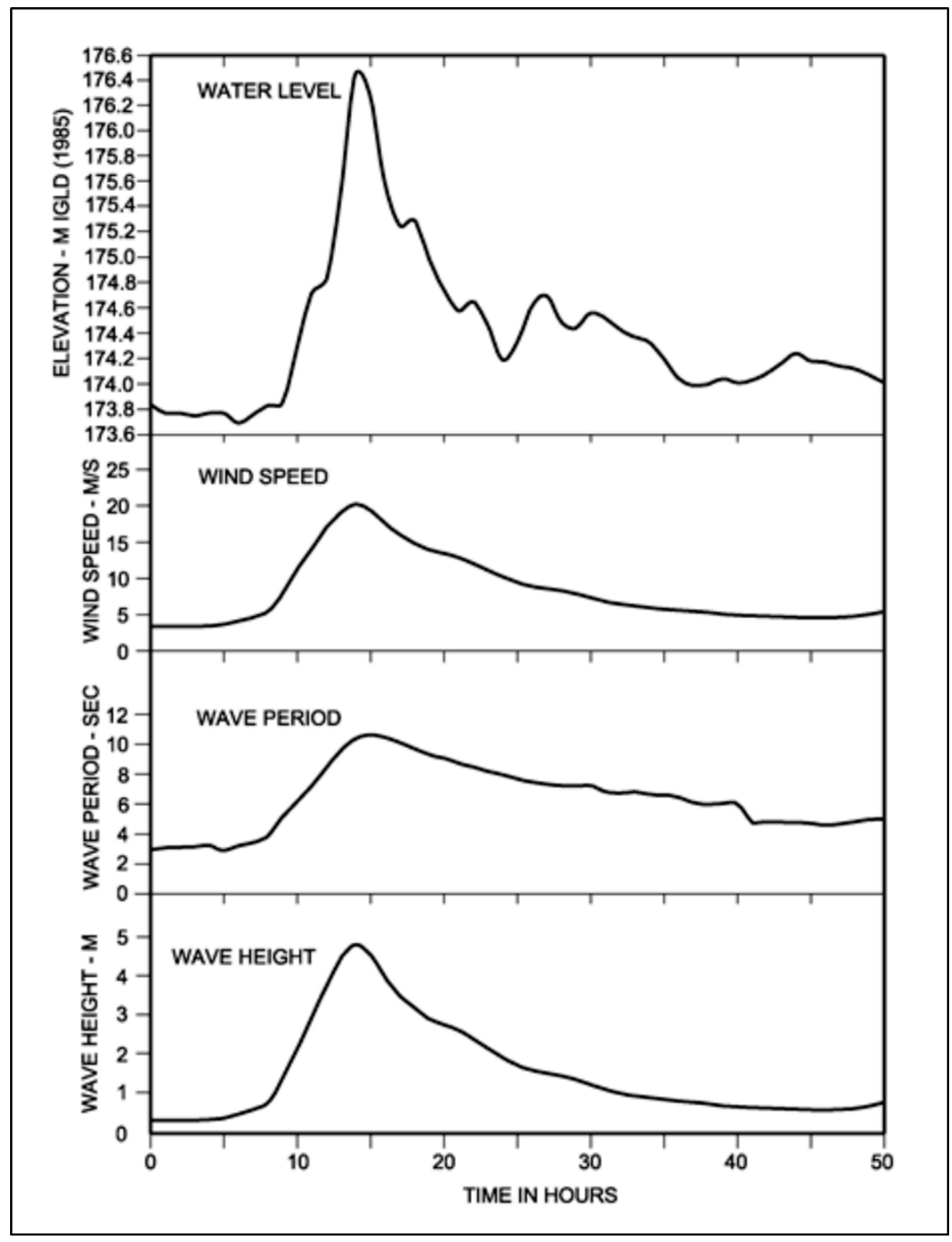


Figure 2-14. The 11 January 1982 storm (Storm Condition 3).

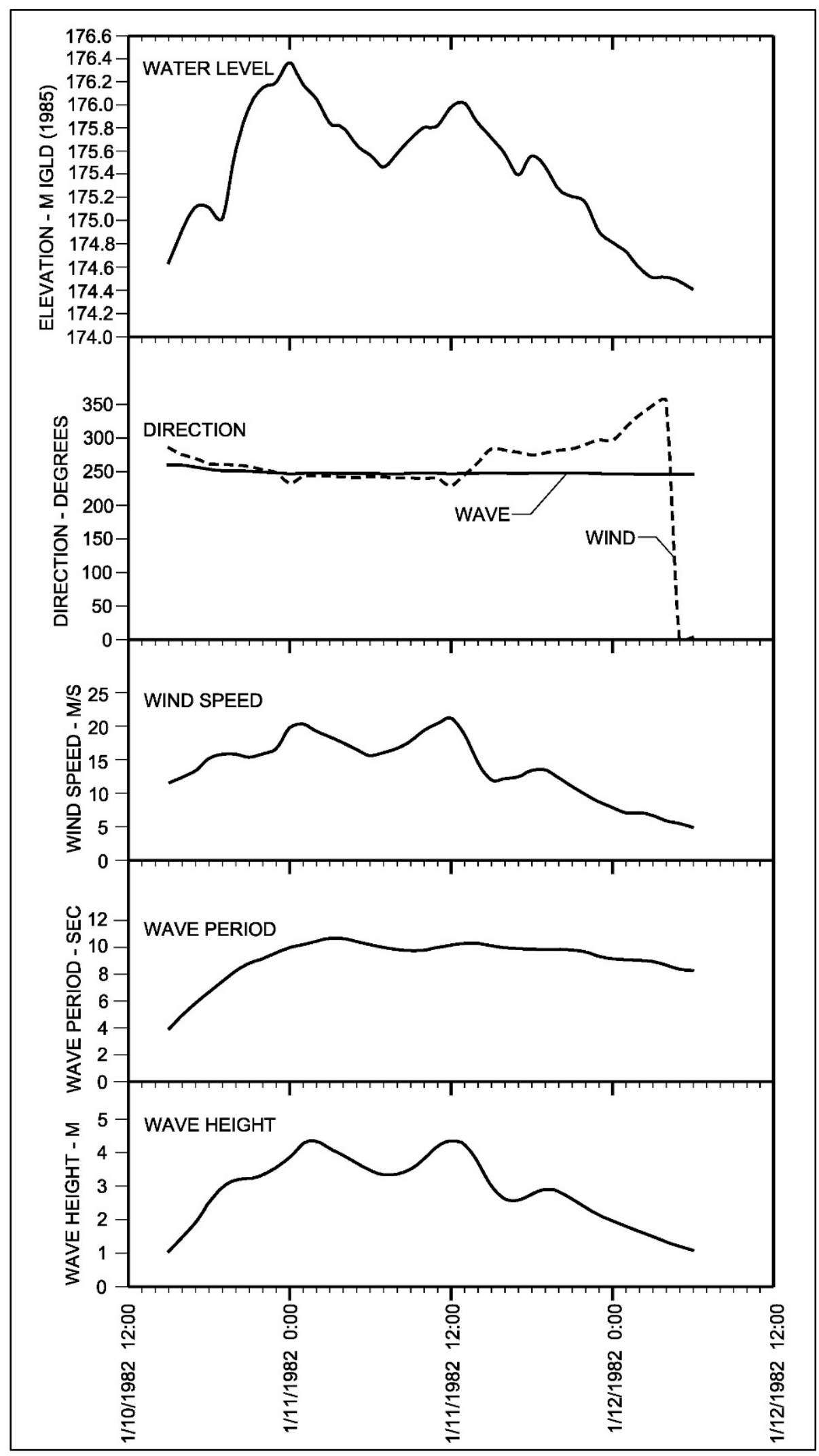




\section{CMS-Wave Modeling}

\subsection{Description of wave models}

Two classes of wave models, BOUSS-2D (B2D) and CMS-Wave, were used to investigate the storm wave effect to $\mathrm{CDF}_{4}$. $\mathrm{B} 2 \mathrm{D}$ is a Boussinesq-type $2 \mathrm{D}$ wave model (Demirbilek and Nwogu 2007; Demirbilek et al. 2007a,b,c; Demirbilek et al. 2005a,b; Nwogu and Demirbilek 2001). CMS-Wave is a steady-state 2D spectral wave model CMS-Wave (Lin and Demirbilek 2012; Lin et al. 2011a,b; Demirbilek and Rosati 2011; Lin et al. 2008; Demirbilek et al. 2008). CMS-Wave is used to transform deep or intermediate-water incident waves to nearshore to develop input conditions for B2D. CMS-Wave is part of an integrated CMS for coastal inlet navigation and regional sediment modeling applications. Additional information about CMS-Wave is provided in Appendix A.

As a nonlinear, time-domain wave model capable of representing various wave processes occurring from deep to shallow water, B2D is a computationally resource-demanding model. Large domain modeling around this harbor was not possible with $\mathrm{B} 2 \mathrm{D}$ because of the large number of conditions to be simulated. It was necessary to augment B2D modeling with a spectral wave model capable of providing wave estimates over a large domain for many wave conditions using CMS-Wave. Because of complementary features of B2D and CMS-Wave, these two models are frequently used in tandem in coastal studies. CMS-Wave was used to transform the offshore waves to the seaward boundary of B2D. Appendix B provides additional information about the $\mathrm{B} 2 \mathrm{D}$ model, its capabilities, and example applications. Details of the B2D modeling for this study are presented in Chapter 4.

CMS-Wave can be used in half-plane or full-plane mode to transform offshore waves to nearshore project sites. The half-plane is the default mode for CMS-Wave, and the model runs efficiently in this mode as waves are transformed primarily from deep water toward shore. CMS-Wave is based on the wave-action balance equation that includes wind-wave generation and growth, wave propagation, refraction, shoaling, diffraction, reflection, breaking, and dissipation. The computational efficiency of CMS-Wave and recent improvements to the capabilities of the model (Lin and Demirbilek 2012; Lin et al. 2011; Demirbilek and Rosati 2011) allow for simulating large spatial domains and a large number of wave conditions in coastal engineering applications. The advantages also include dynamic coupling 
with CMS-Flow, a circulation and sediment transport model in the CMS, and the use of nesting of multiple grids for simultaneous modeling of regional and local processes. The remainder of this chapter is focused on detailed information about CMS-Wave modeling for $\mathrm{CDF}_{4}$.

\subsection{Type and condition of structures}

The Perimeter Dike was designed as a sediment filtering structure and the SEAB as an impermeable structure due to its ashlar construction. As previously indicated, damage to the Perimeter Dike has resulted in random crest lowering and sporadic damage to the upper steel sheet pile cutoff wall. Partial restoration of the crest has occurred as funding allowed, but the filtering capacity has been maintained by grouting locations where ice forces raised the steel sheet pile out of the filter layer. The SEAB is in a condition similar to when it was originally constructed. Internal water levels and waves are a function of lake levels, wave overtopping, and structure permeability. The contribution and sensitivity of the system permeability was investigated by considering the following three scenarios: (1) both dike and breakwater are treated as permeable, (2) only the dike is treated as permeable, and (3) both dike and breakwater are treated as impermeable. For the permeable structure considered in (1) and (2), a low permeability of wave and flow transmission through the structures was assumed.

\subsection{Modeling grids and domains}

A regional grid (parent grid) and a local $\mathrm{CDF}_{4}$ grid (child grid) were developed in the present study for CMS-Wave. The parent grid includes Buffalo Harbor and CDF4 with the offshore boundary extended westward to the WIS station 92243. The child grid with a smaller domain and finer resolution includes the $\mathrm{CDF}_{4}$ and neighboring nearshore and channel areas.

The CMS-Wave parent grid covered a rectangular area approximately 7 miles $\times 11$ miles $(11 \mathrm{~km} \times 17 \mathrm{~km})$. The grid cell size in the parent grid varied from 65 to $650 \mathrm{ft}$ ( 20 to $200 \mathrm{~m}$ ) and water depths o to $48 \mathrm{ft}$ (o to $14.6 \mathrm{~m}$ ). The parent grid was used to transform the offshore incident wave to the CDF4 nearshore to develop and provide the wave condition for the CMSWave child grid and B2D grid. The CMS-Wave child grid covered a smaller rectangular area approximately 1 mile $\times 1.25$ miles $(1.5 \mathrm{~km} \times 2 \mathrm{~km})$. The child grid cell size varied from 10 to $100 \mathrm{ft}$ ( 3 to $30 \mathrm{~m}$ ) with smaller cells around the $\mathrm{CDF} 4$. Figure 3-1 shows the CMS-Wave parent grid domain (red box) and child grid domain (yellow box). Figure 3-2 shows a closer view of the CMS-Wave child grid domain and bathymetry around CDF4. 
Figure 3-1. CMS-Wave parent grid domain (red box) and child grid domain (yellow box).

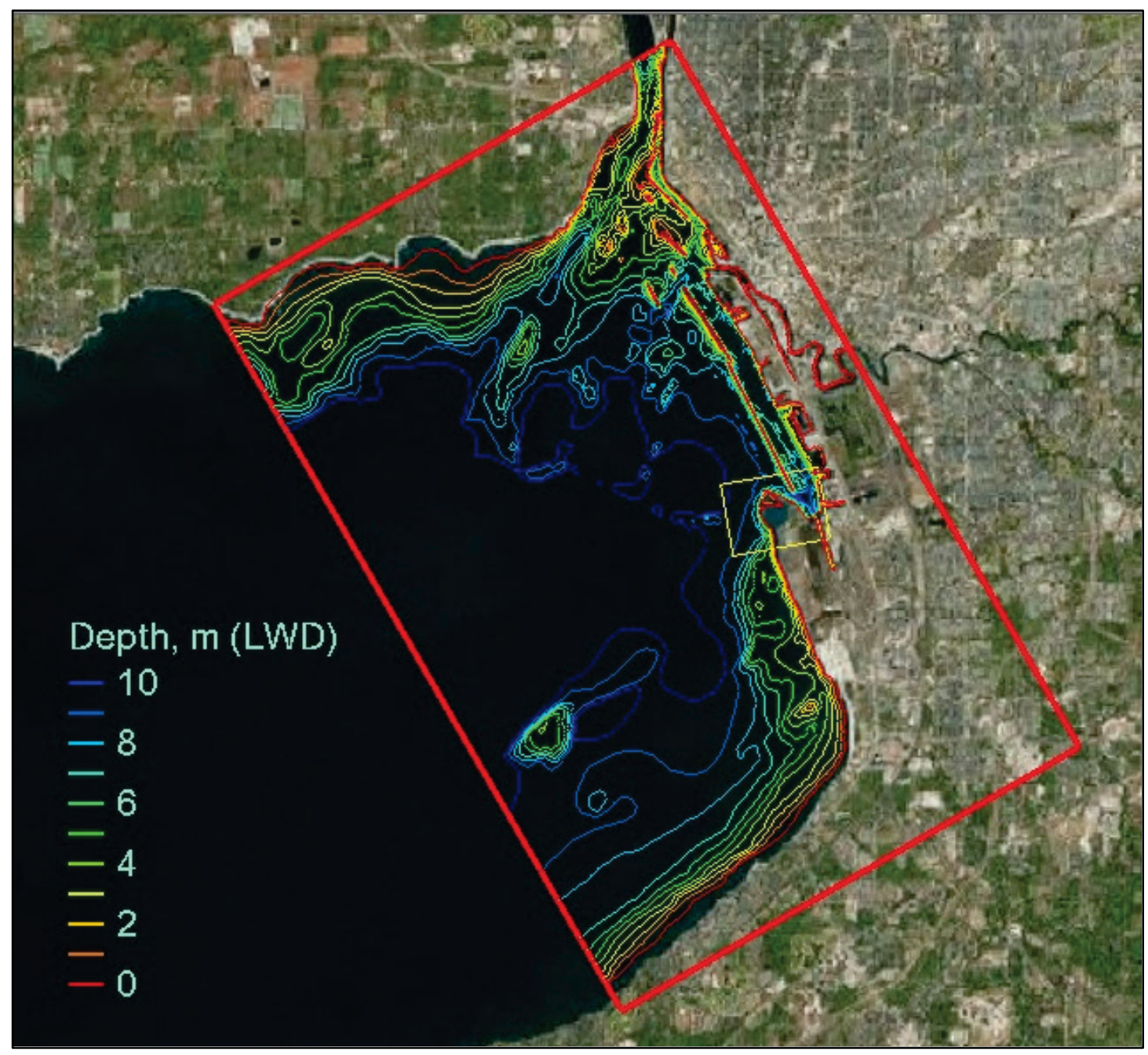


Figure 3-2. CMS-Wave child grid domain (yellow box) with bathymetry contours.

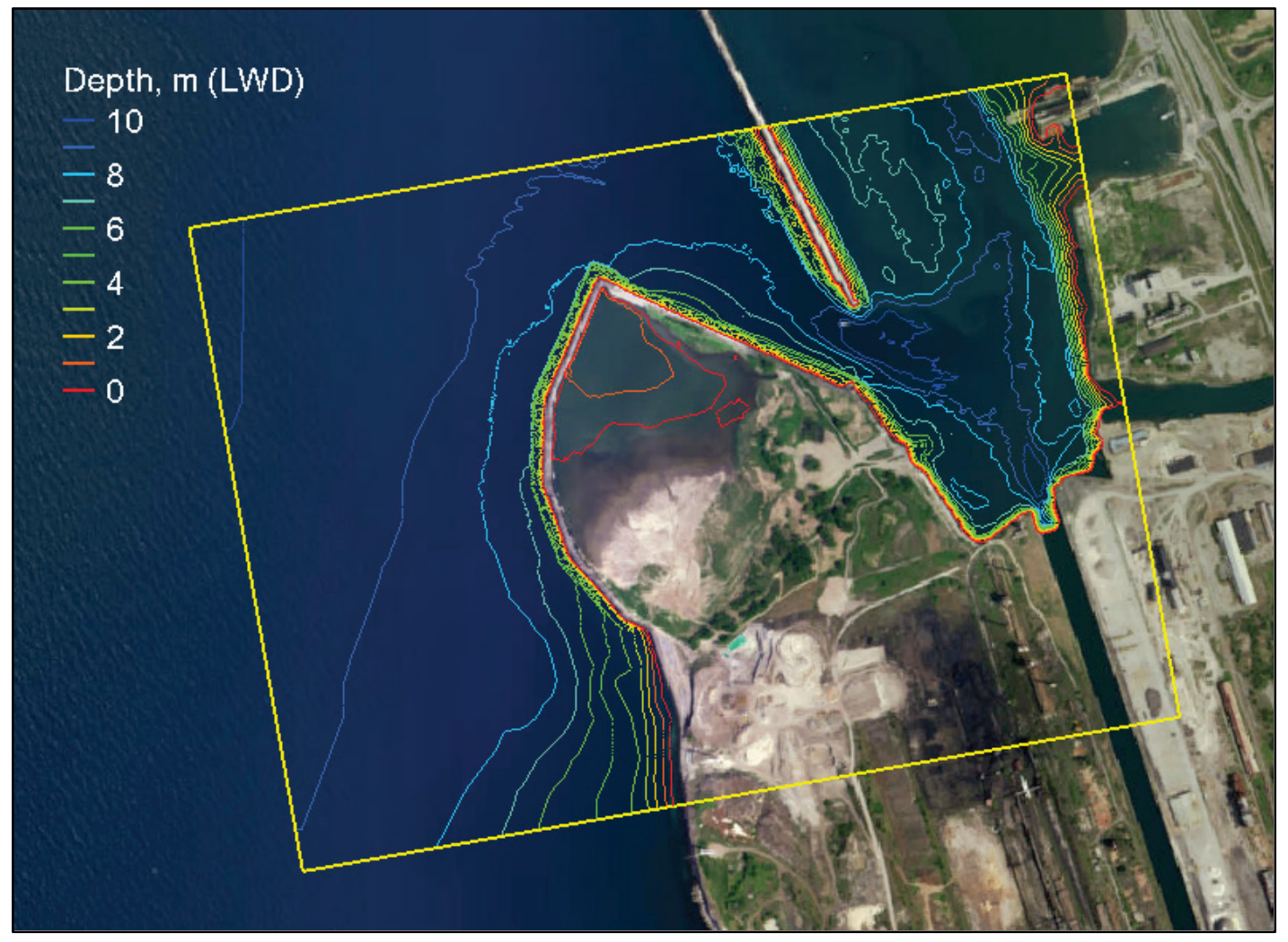

\subsection{Model settings}

As described in Section 2.5, three storm conditions were selected to force the wave models: (1) 2-year wave and 2-year water level (Storm Condition 1), (2) 20-year wave and 10-year water level (Storm Condition 2), and (3) the actual 11 January 2008 storm representing a typical average storm wave event in the region (Storm Condition 3). A constant wave direction of 247 deg was used for Storm Conditions 1 and 2.

CMS-Wave was run for the grid nesting of a parent grid and a child grid in the half-plane mode. The vertical datum used in the model is the LWD, defined as $569.2 \mathrm{ft}(173.5 \mathrm{~m})$ IGLD 1985. IGLD 1985 has its zero base at Rimouski, Quebec, near the mouth of the Saint Lawrence River (approximate sea level). The parent grid simulation provides the wave forcing boundary condition to the child grid. Wave spectral transformation was computed in CMS-Wave using 40 frequency bins (covering the range of 0.06 to 0.45 hertz [Hz] at $0.01 \mathrm{~Hz}$ increment) and 35 direction bins (covering a half-plane for incoming wave direction in the range of 152.5 to 327.5 deg azimuth with 5 deg increment). Wave runup and infra-gravity wave options were activated in the parent and child grid simulations. The 
diffraction intensity was set to 4 (default) for the maximum diffraction allowed in CMS-Wave. A constant Darcy-Weisbach coefficient of 0.005 and a constant reflection coefficient of 0.5 were used for bottom friction and forward reflection calculations, respectively. For the permeable structure, a low-porous (semi-permeable) structure is specified for the structure section above the LWD.

To include the flow and water level forcing in the CMS-Wave modeling to calculate the wave overtopping rate for $\mathrm{CDF}_{4}, \mathrm{CMS}$-Wave was coupled with the CMS-Flow. The CMS-Flow 2D explicit-solver version of the model was used for hydrodynamics modeling. A ponding routine developed for this CMS-Flow version was used to specify the initial water level present inside the CDF4. CIRP provided funding for developing and testing this new capability for the explicit flow model. Wind, wave, water level, and river discharge forcings were included in the CMS-Flow simulation. The annual average river discharge of $204,000 \mathrm{ft} 3 / \mathrm{sec}(5,777 \mathrm{~m} 3 / \mathrm{sec})$ was specified for the Niagara River and $700 \mathrm{ft} 3 / \mathrm{sec}(20 \mathrm{~m} 3 / \mathrm{sec})$ for the Buffalo River. For the permeable structure, a low permeability of 0.05 was used for the structure layer above the LWD. The computational time-step in CMS-Flow is $0.25 \mathrm{sec}$. CMS-Flow was run on the same parent and child grids as used in CMS-Wave. The current and water level fields computed from CMS-Flow were used as hydrodynamic forcing to CMS-Wave while the wave radiation stress fields computed from CMS-Wave were used as wave forcing to CMS-Flow. CMS-Wave and CMS-Flow were coupled at $1 \mathrm{hr}$ intervals. The wave radiation stresses computed from two consecutive CMS-Wave runs were linearly applied in the CMS-Flow simulation.

\subsection{Model simulations}

CMS-Wave was run and coupled with CMS-Flow on the parent and child grids for three storm conditions. For each storm condition, simulations were conducted for either a permeable or impermeable structure, or permeable dike. The model water level was mainly controlled by the storm surge forcing applied along the open boundaries. The water level inside the CDF4 was initially set to the LWD level (ponding effect). The CDF4 water level remains at the LWD level until water exchange occurs from wave and flow overtopping the dike or through the permeable structure. Wave model results were saved over the entire computational domain, including three engineering wave parameters (significant wave height, peak period, and mean direction). Calculated directional spectra were saved at 68 and 63 output stations (i.e., save locations) along the $\mathrm{CDF}_{4}$ structure in the parent 
and child grids, respectively. The distance between two neighboring save locations is approximately $82 \mathrm{ft}(25 \mathrm{~m})$.

Figure 3-3 shows seven spectral wave output locations (yellow points) for boundary condition forcing to the child grid (brown box) and 68 save locations (black points) in the parent grid. Figure 3-4 shows 14 transect lines (T1 to T14) and 63 save locations (black points) in the child grid. Figures 3-5 to $3-8$, for example, show the landward hydrographic profiles along $\mathrm{T}_{4}-\mathrm{T}_{5}$, T6-T7, T8-T10, and T11-T14, respectively. The crest elevations of the dike at T6-T7 (Leg 4) and T8-T10 (Leg 5) are lower than elevations at T4-T5 (Leg 3) as that crest was recently repaired, and $\mathrm{T} 11-\mathrm{T} 14$ (Leg 6) indicates that its original crest was raised by the addition of cut stone blocks as discussed previously in Section 2.2

Figure 3-3. Parent grid; 7 spectral output (yellow dots) and 68 save locations (black dots).

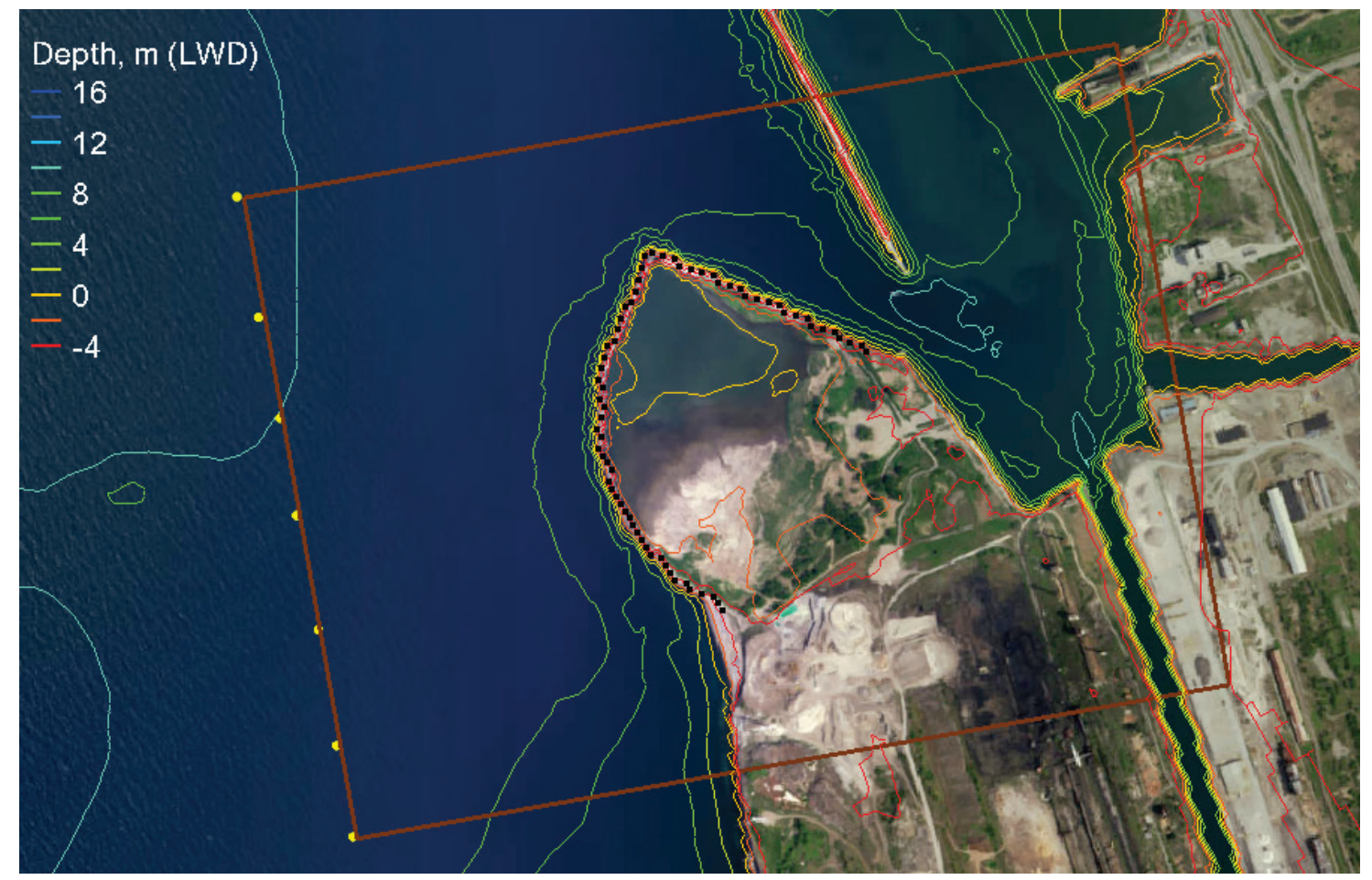


Figure 3-4. Child grid; 14 transect lines (T1 to T14) and 63 save locations (black dots).

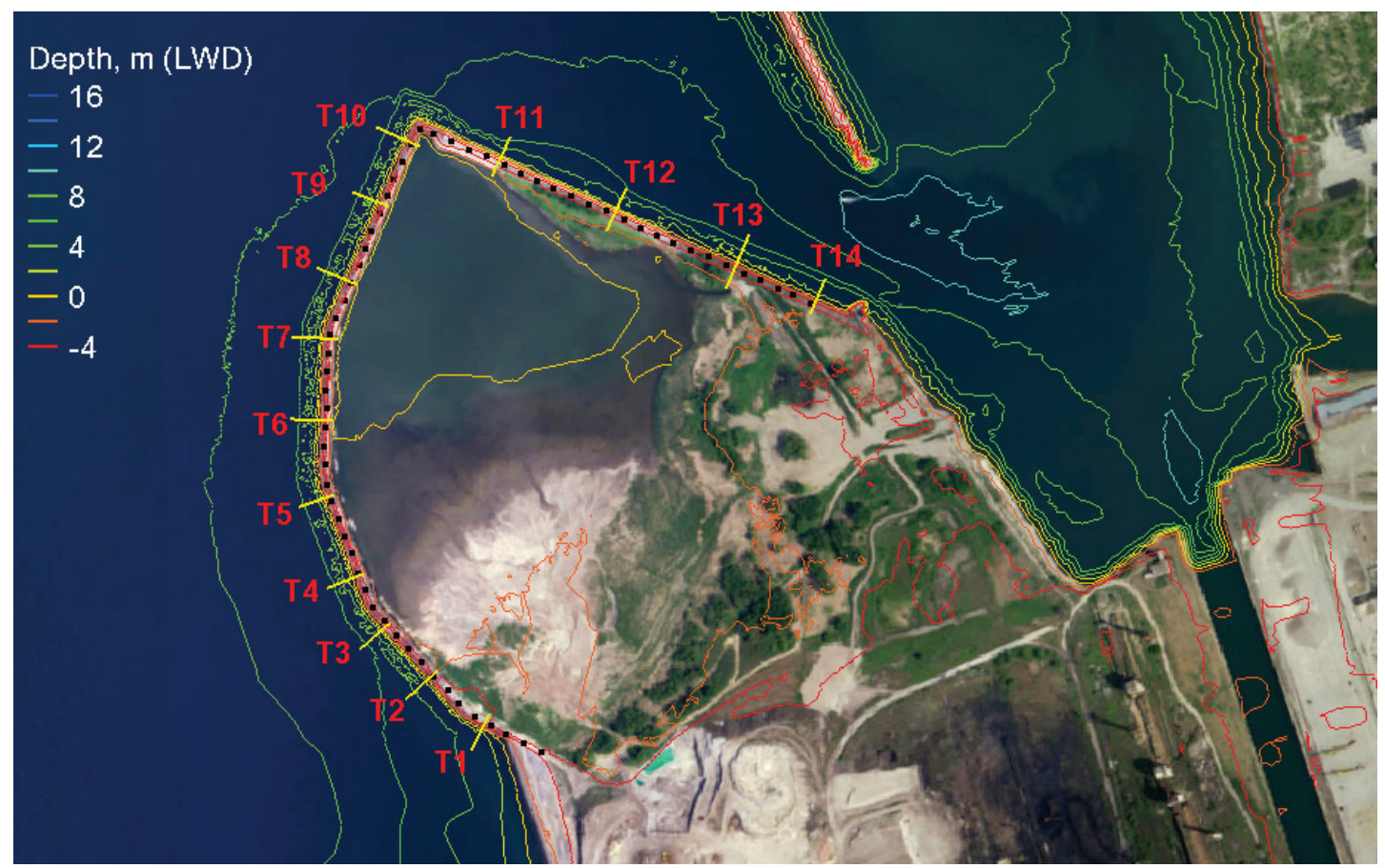

Figure 3-5. Profiles of transects T4 and T5.

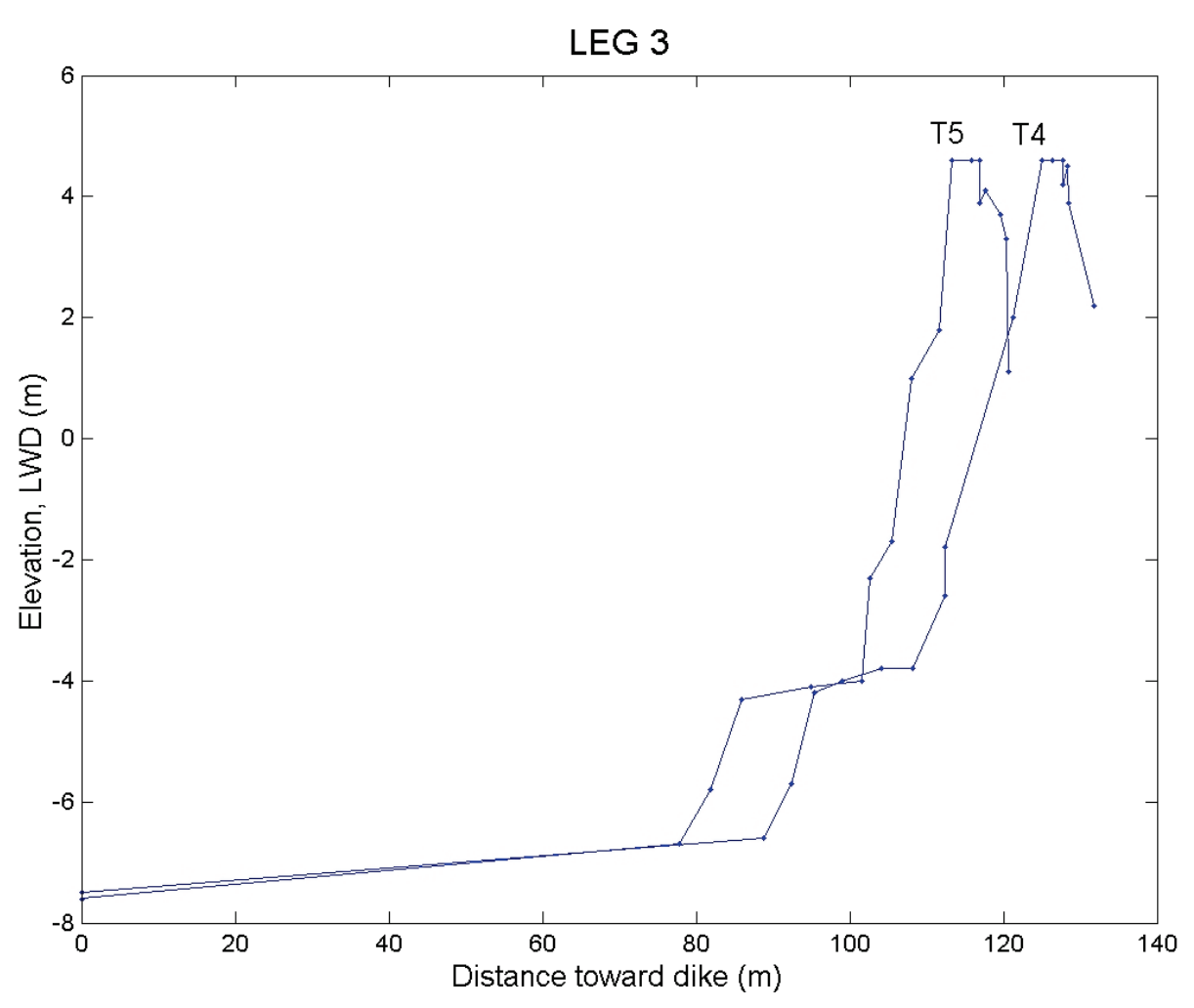


Figure 3-6. Profiles of transects T6 and T7.

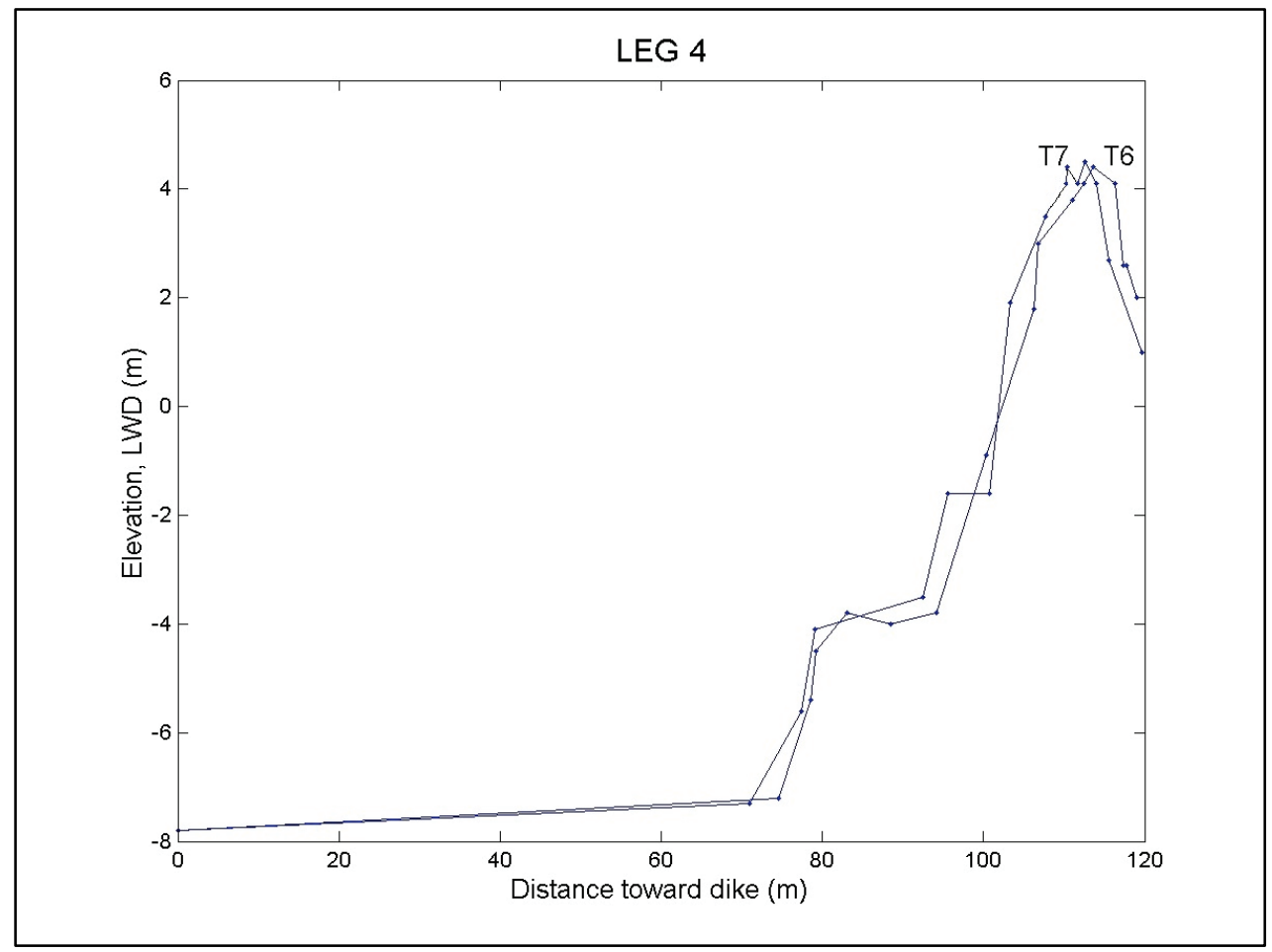

Figure 3-7. Profiles of transects T8, T9, and T10.

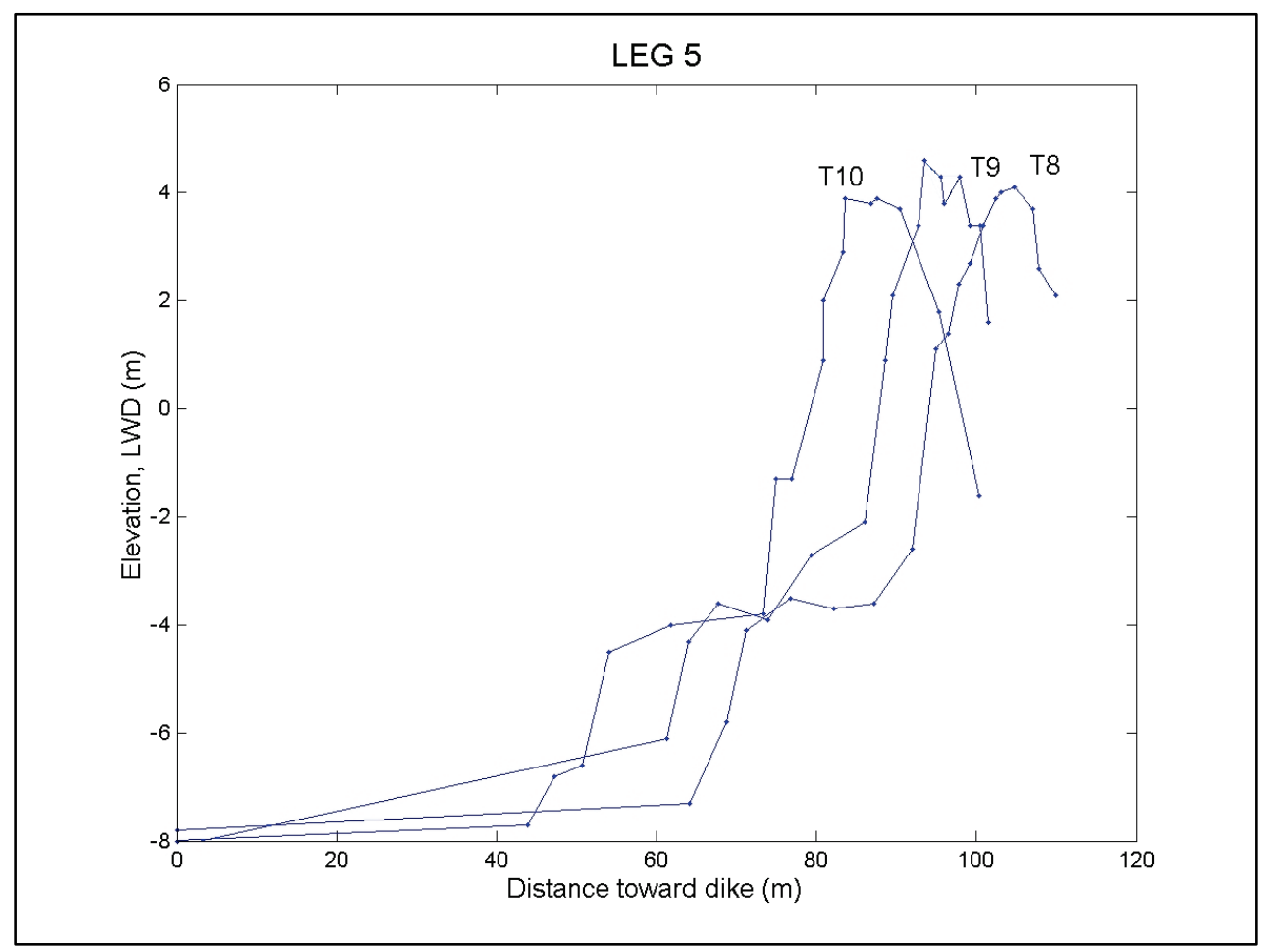


Figure 3-8. Profiles of transects $\mathrm{T} 11, \mathrm{~T} 12, \mathrm{~T} 13$, and T14.

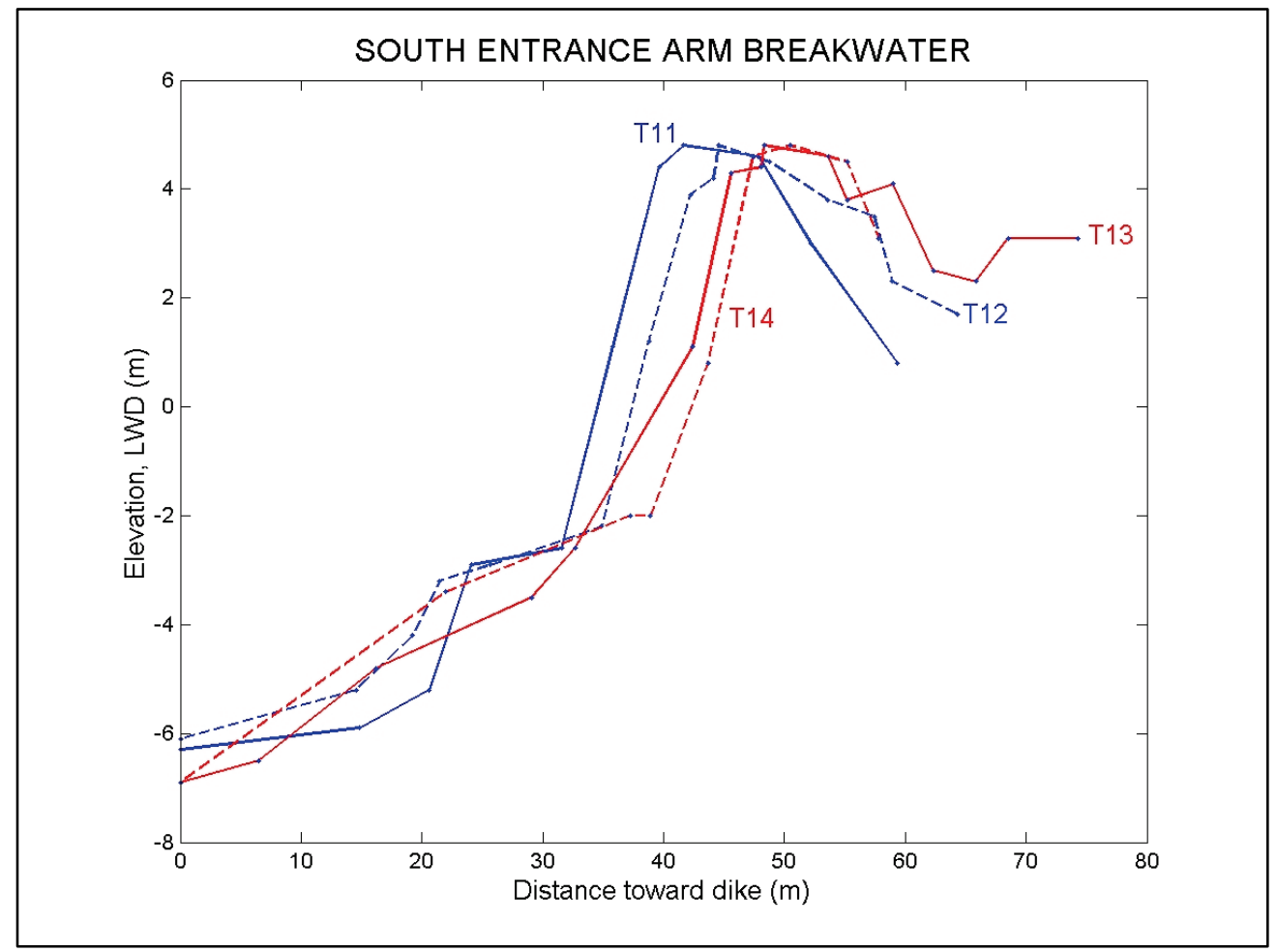

Figures 3-9 and 3-10 show, for example, calculated maximum wave field and corresponding current fields, respectively, at 12:00 Greenwich Mean Time (GMT), 11 January 1982, from Storm Condition 3, on the parent grid.

Figures 3-11 to 3-13 show calculated wave and current fields at the same time (12:00 GMT, 11 January 1982) on the child grid for the permeable structure, permeable dike, and impermeable structure conditions, respectively. 
Figure 3-9. Calculated wave field 11 January 1982; 12:00 GMT.

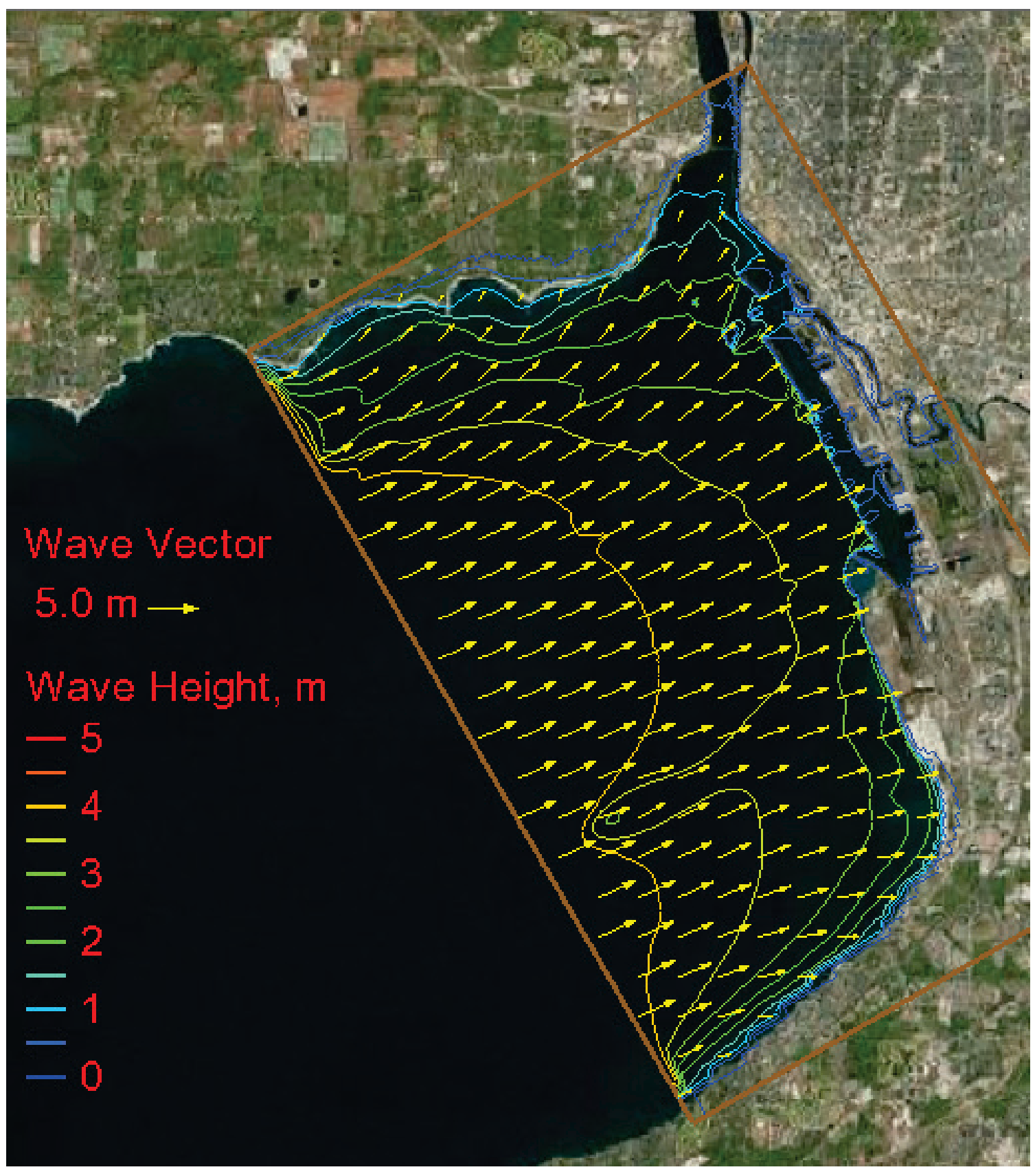


Figure 3-10. Calculated water level/current field 11 January1982; 12:00 GMT.

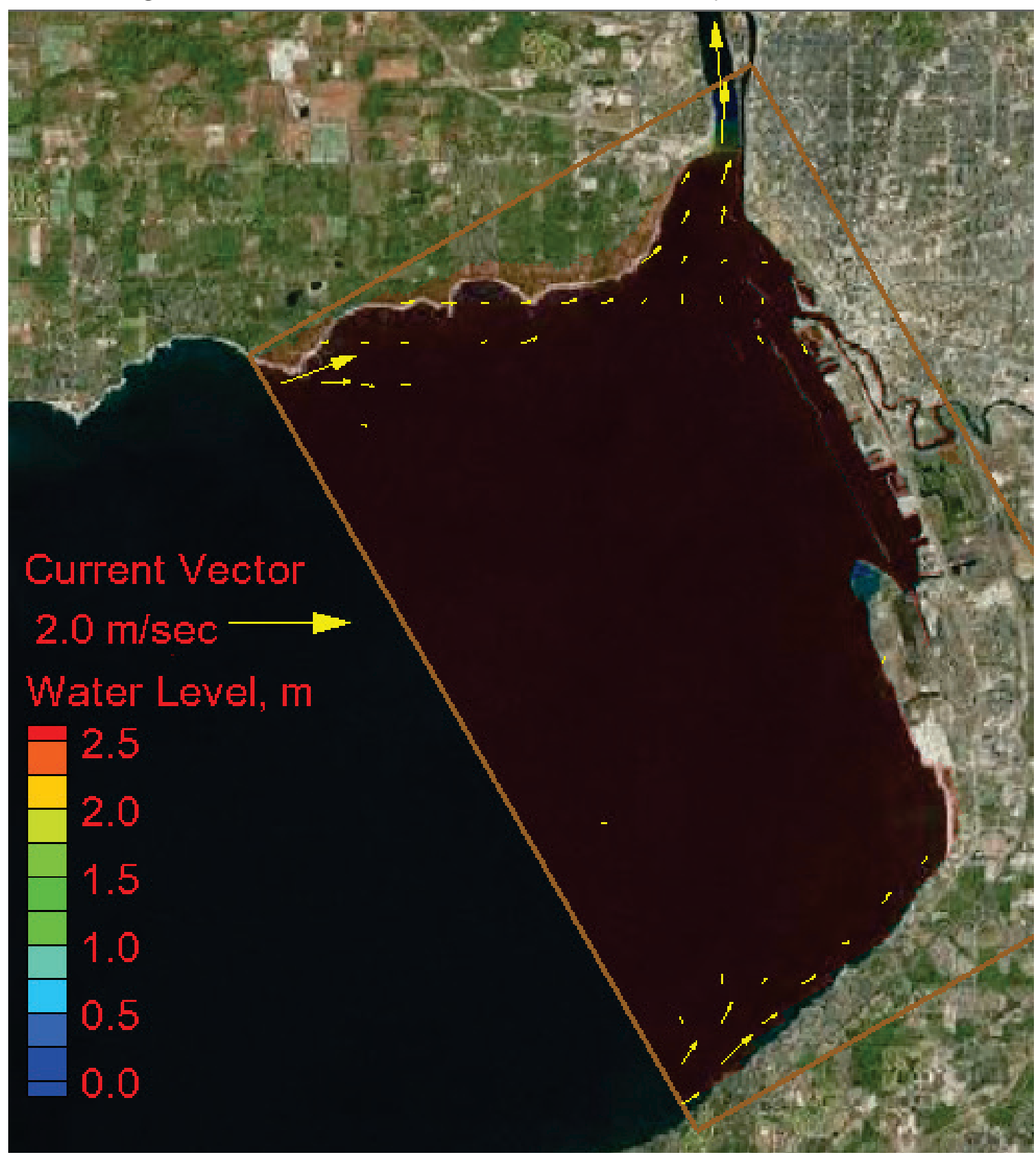


Figure 3-11. Calculated wave/current field with permeable structures 11 January 1982; 12:00 GMT.

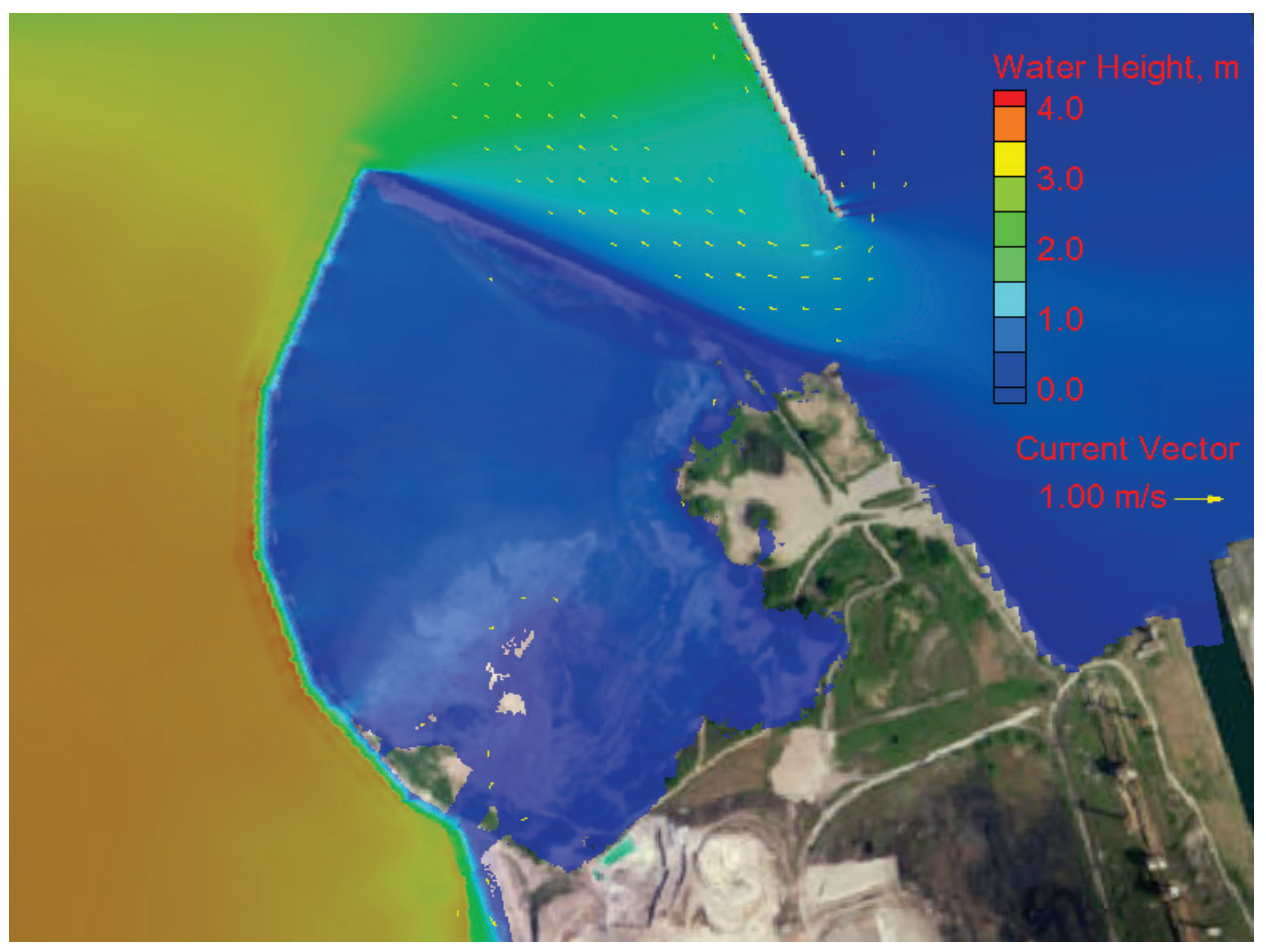

Figure 3-12. Calculated wave/current field with permeable dike 11 January 1982; 12:00 GMT.

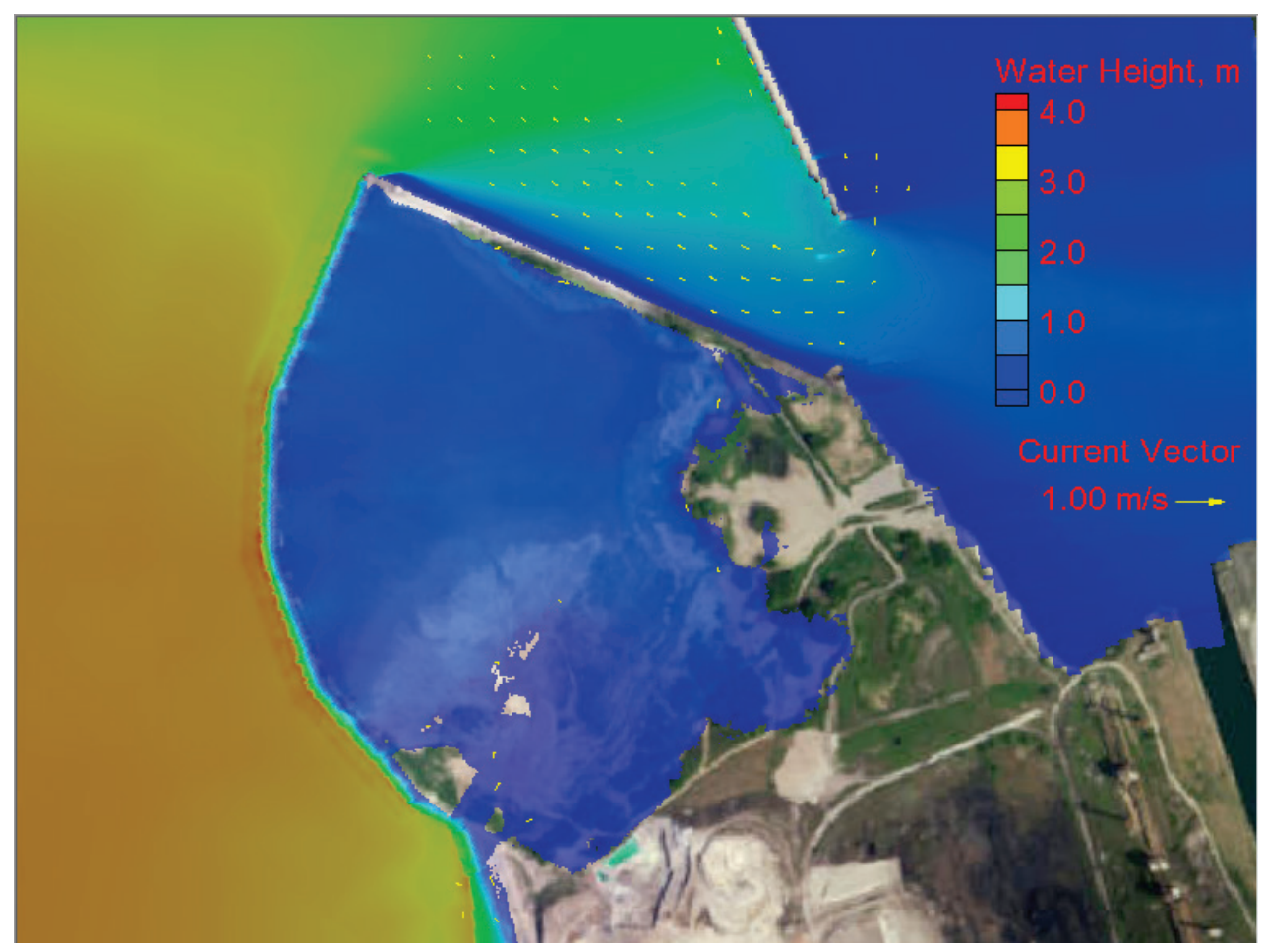


Figure 3-13. Calculated wave/current field with impermeable dike 11 January 1982; 12:00 GMT.

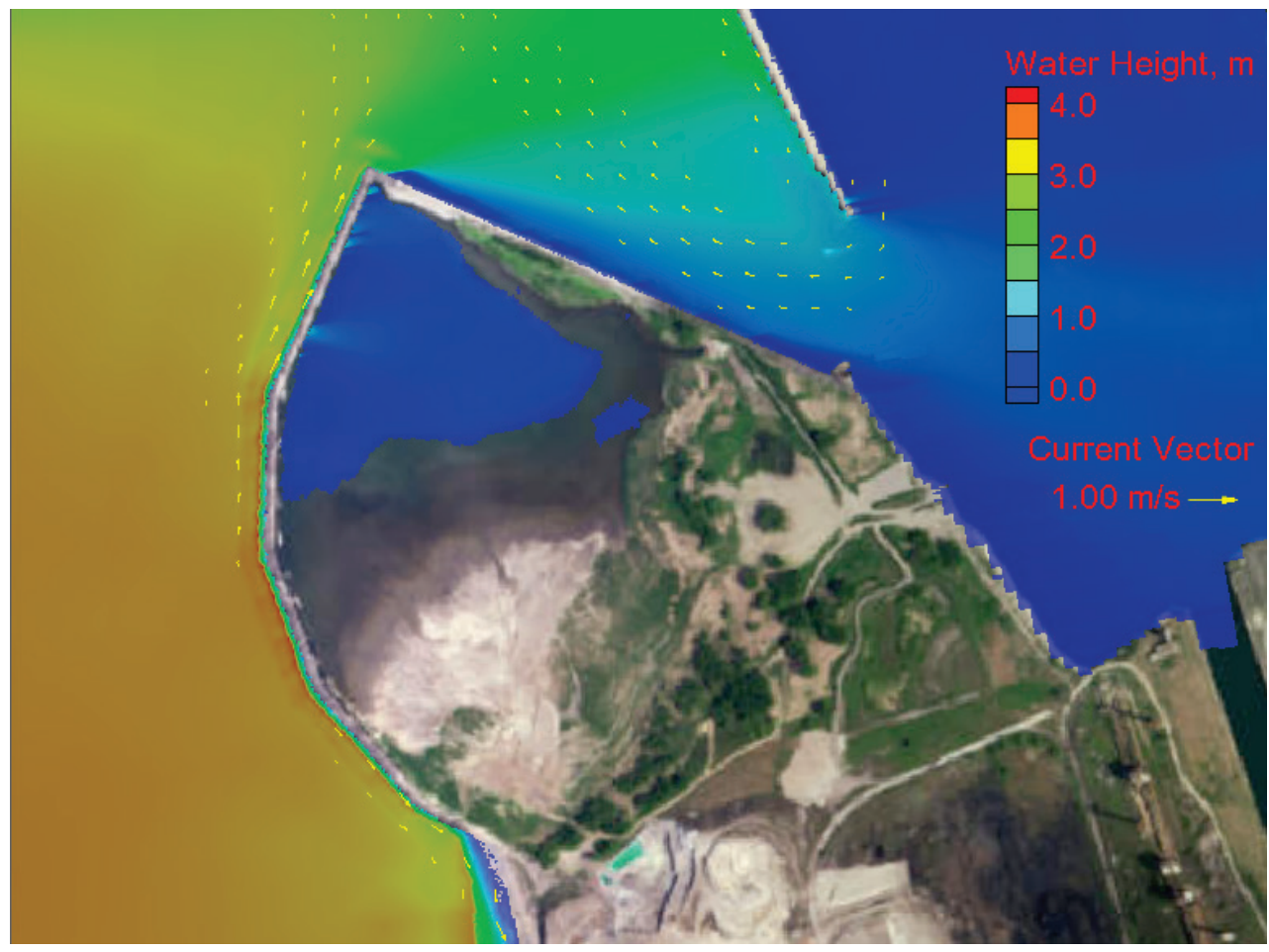

\subsection{Wave overtopping estimates}

The wave overtopping rate was estimated for the permeable structure, permeable dike, and impermeable structure from model simulations. Figures 3-14 to 3-16 show the hourly and total wave overtopping rates along the $\mathrm{CDF} 4$ structure under Storm Condition 1 for the permeable structure, permeable dike, and impermeable structure, respectively. Figures 3-17 to 3-19 show the hourly and total wave overtopping rates along the dike under Storm Condition 2 for the permeable structure, permeable dike, and impermeable dike, respectively. Figures 3-20 to 3-22 show the hourly and total wave overtopping rates along the dike under Storm Condition 3 for the permeable structure, permeable dike, and impermeable structure, respectively. Note vertical scale changes for Figures 3-14 through 3-22. All wave overtopping rates are presented for per unit width. Tables $3-1,3-2$, and 3-3 present the calculated maximum wave overtopping rate and total overtopping volume at the $\mathrm{CDF}_{4}$ structure for Storm Conditions 1, 2, and 3 , respectively. 
Figure 3-14. Hourly and total wave overtopping rates along permeable structures (Storm Condition 1).

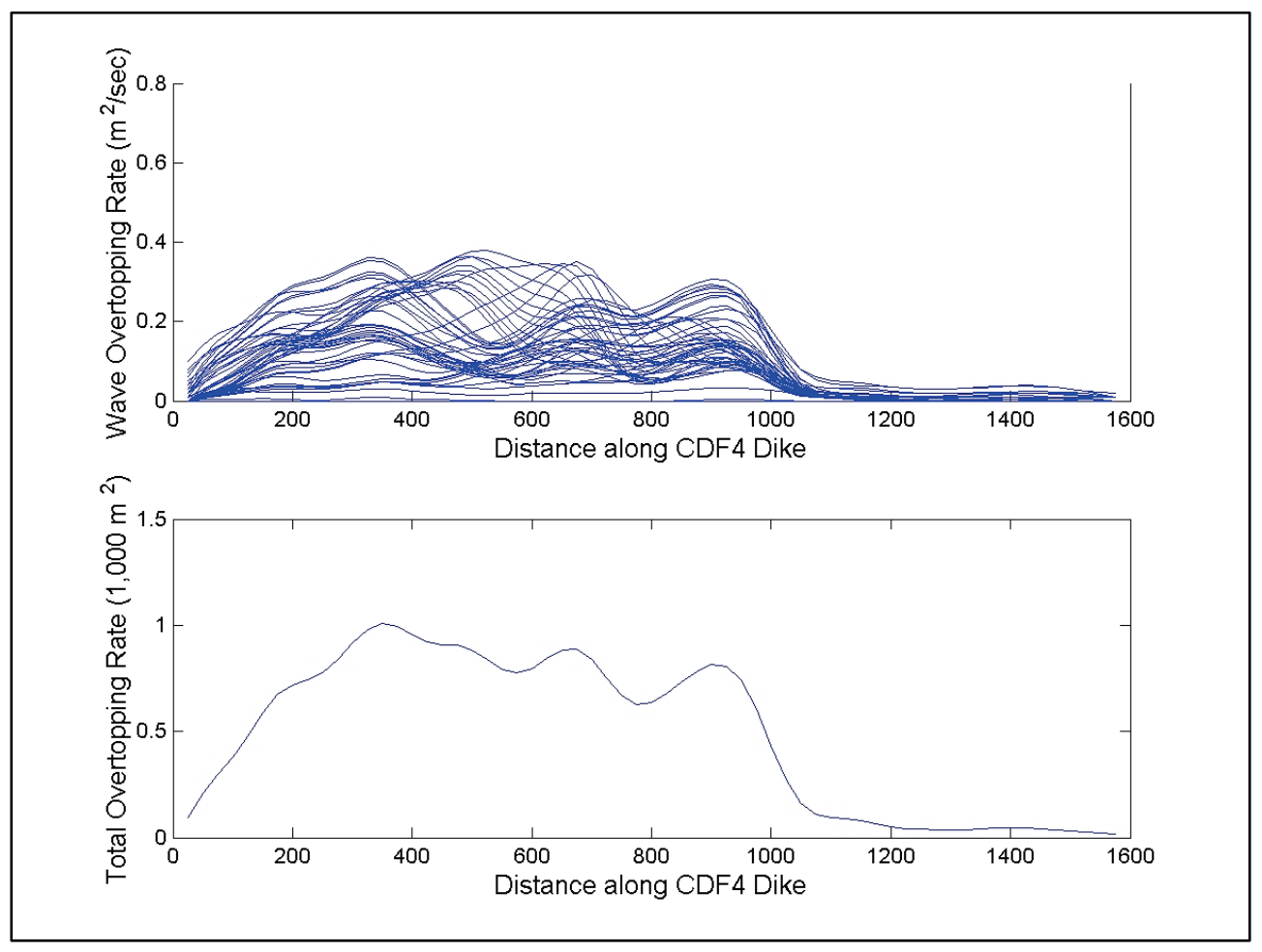

Figure 3-15. Hourly and total wave overtopping rates along permeable dike (Storm Condition 1).

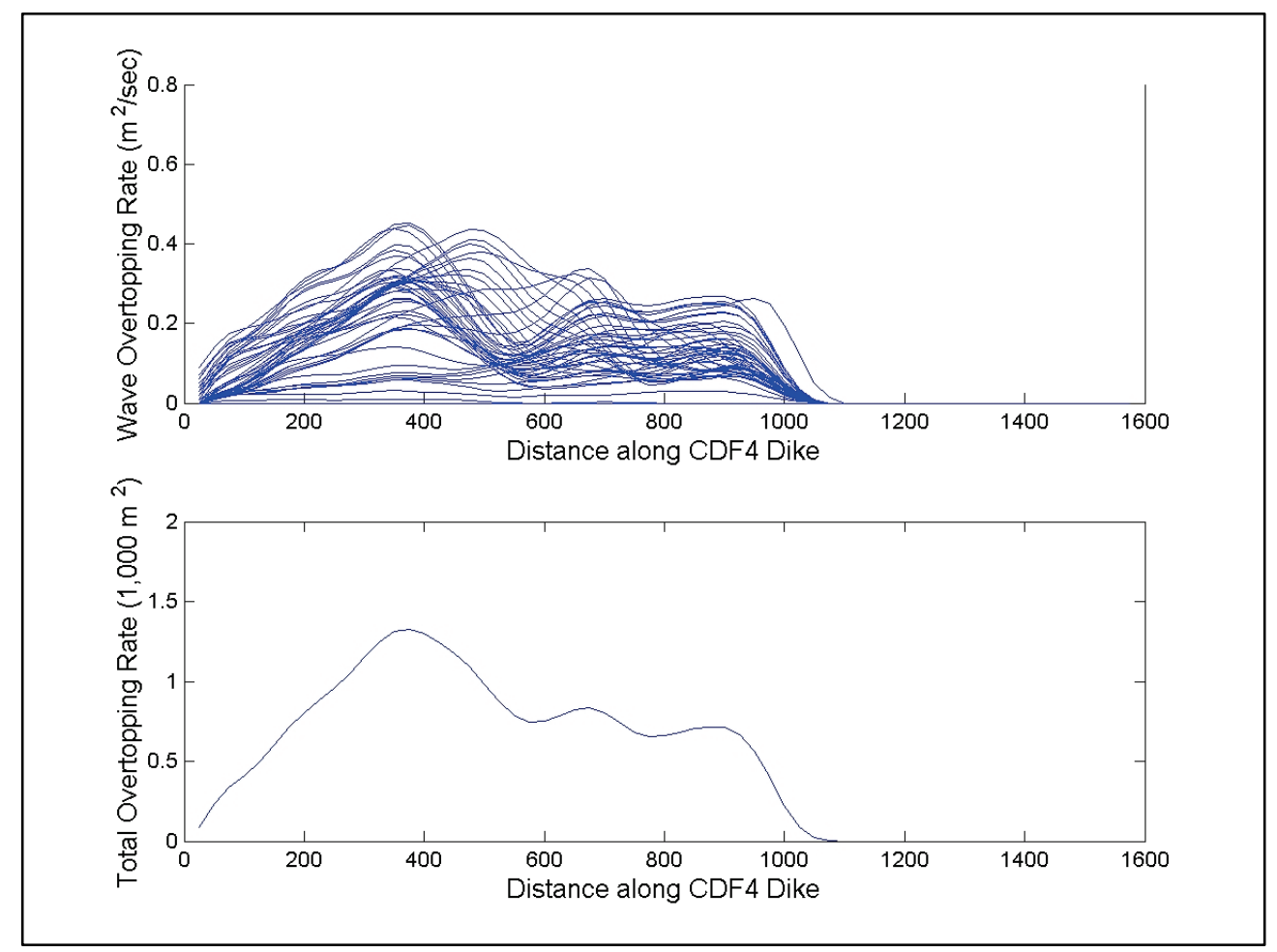


Figure 3-16. Hourly and total wave overtopping rates for impermeable structures (Storm Condition 1).

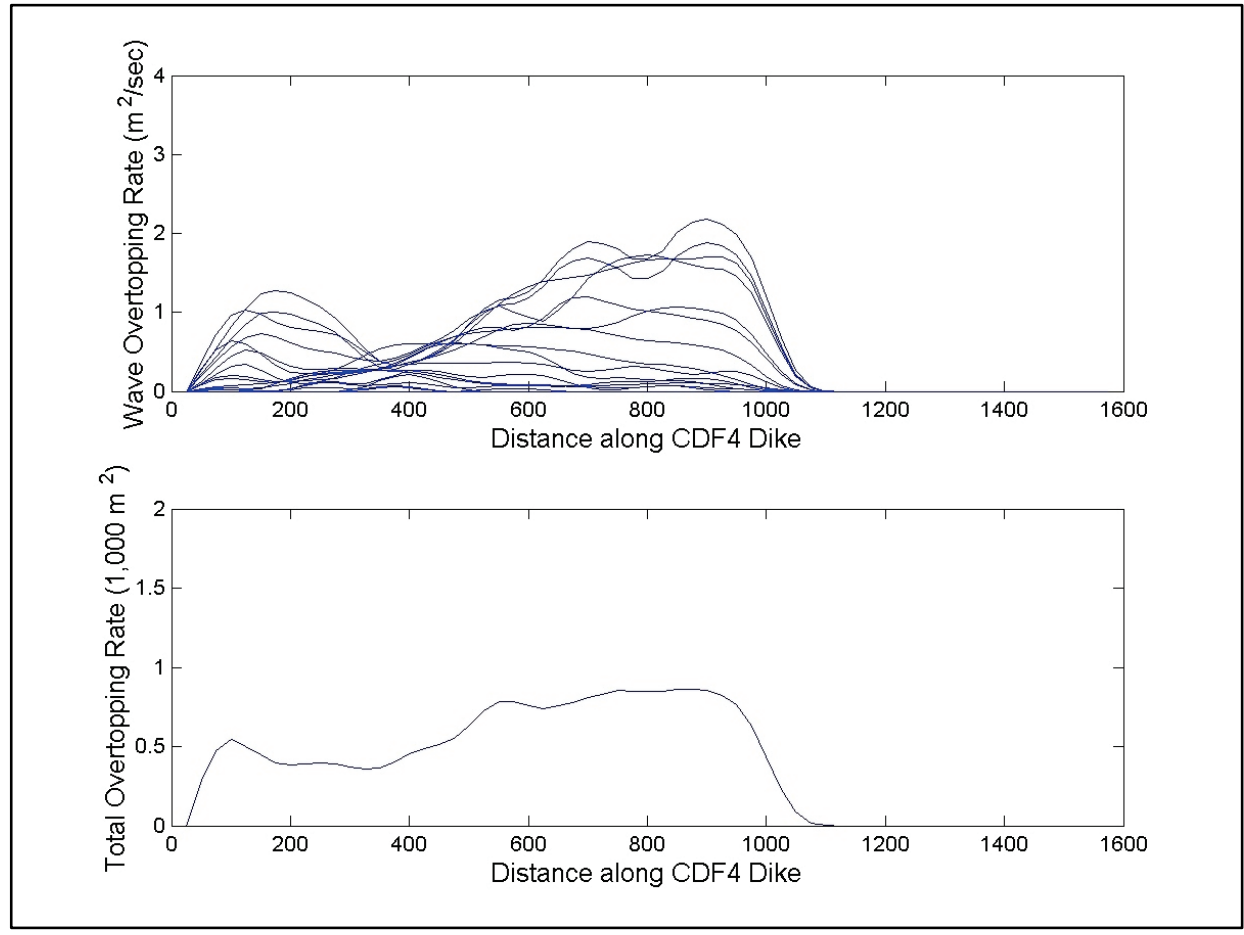

Figure 3-17. Hourly and total wave overtopping rates along permeable structures (Storm Condition 2).
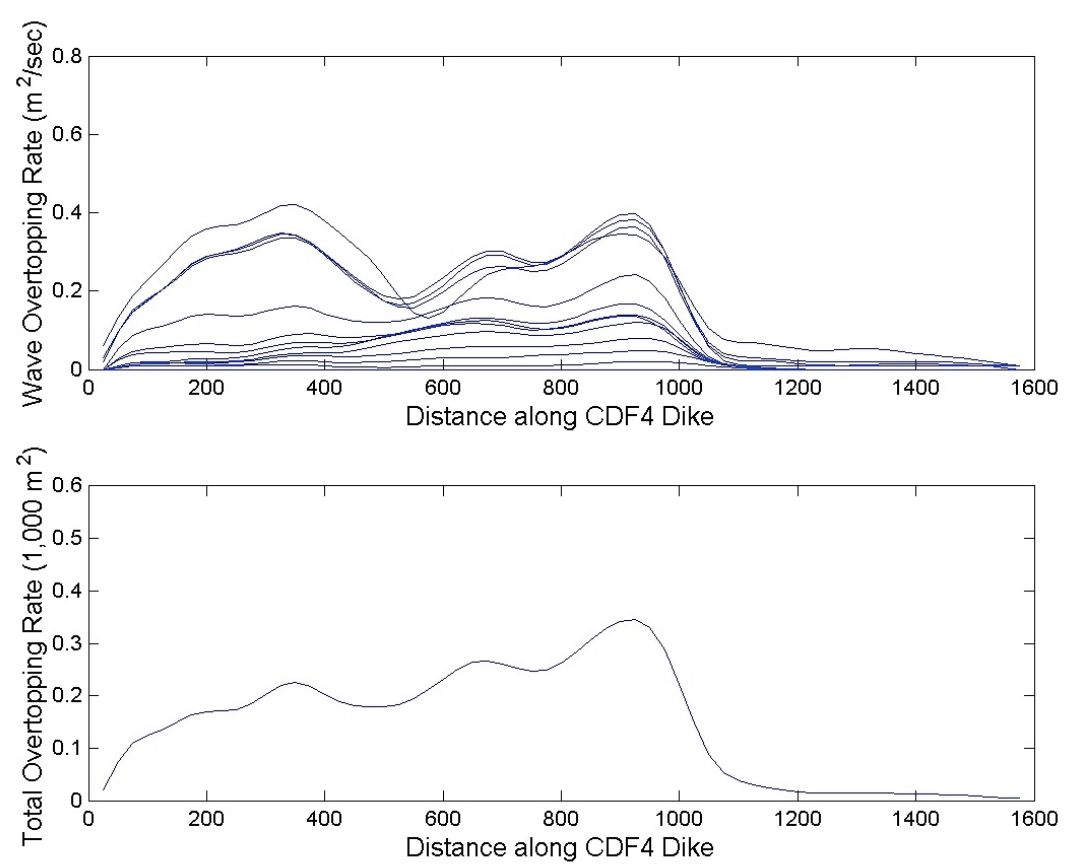
Figure 3-18. Hourly and total wave overtopping rates along permeable dike (Storm Condition 2).

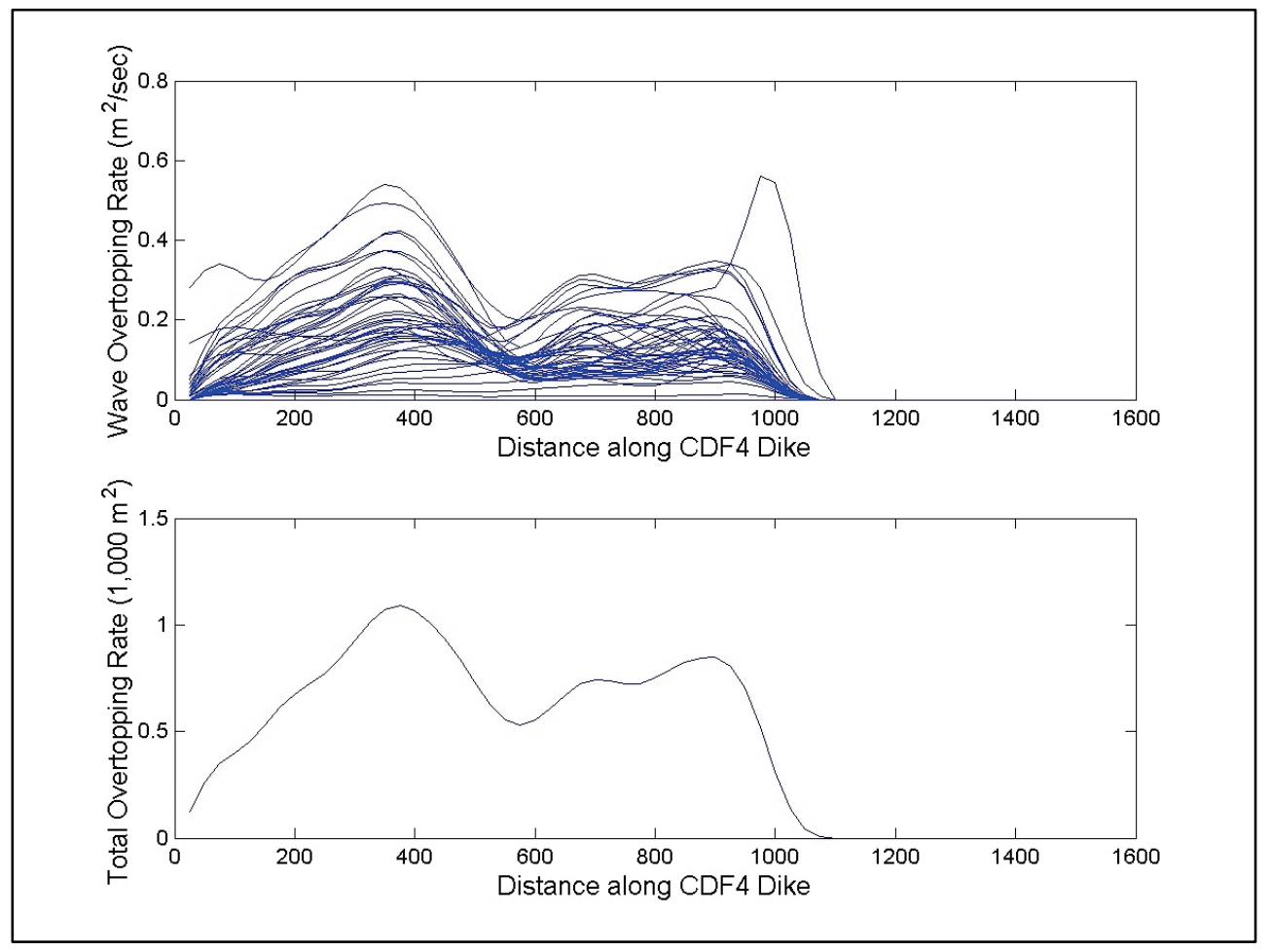

Figure 3-19. Hourly and total wave overtopping rates for impermeable structures (Storm Condition 2).
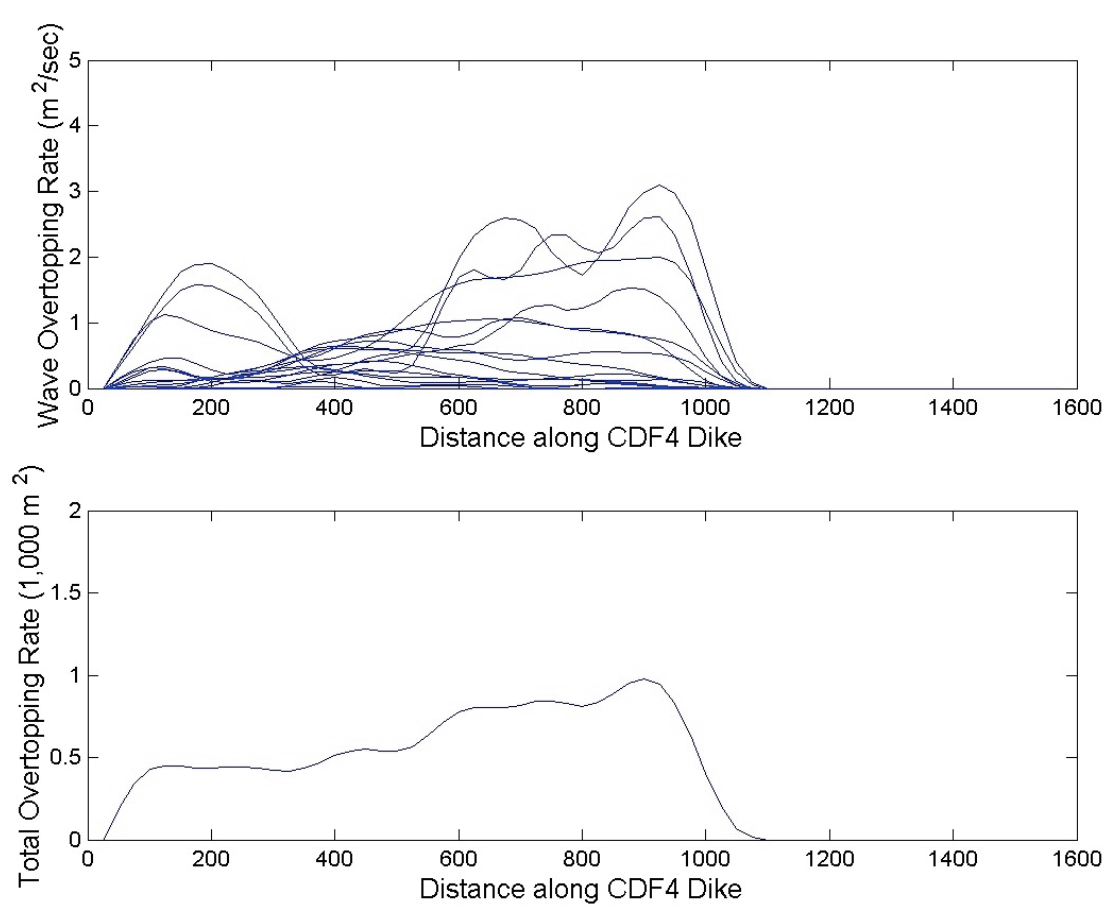
Figure 3-20. Hourly and total wave overtopping rates along the permeable structures (Storm Condition 3).
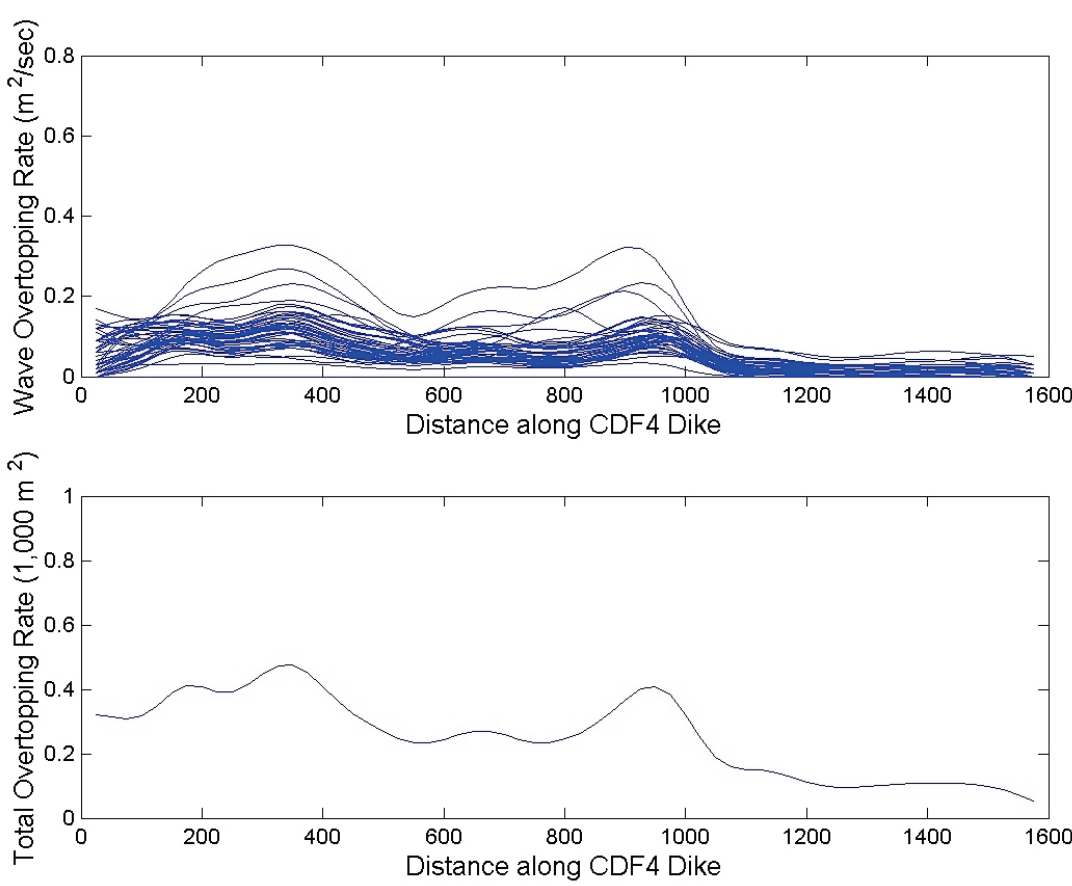

Figure 3-21. Hourly and total wave overtopping rates along the dike for permeable dike (Storm Condition 3).
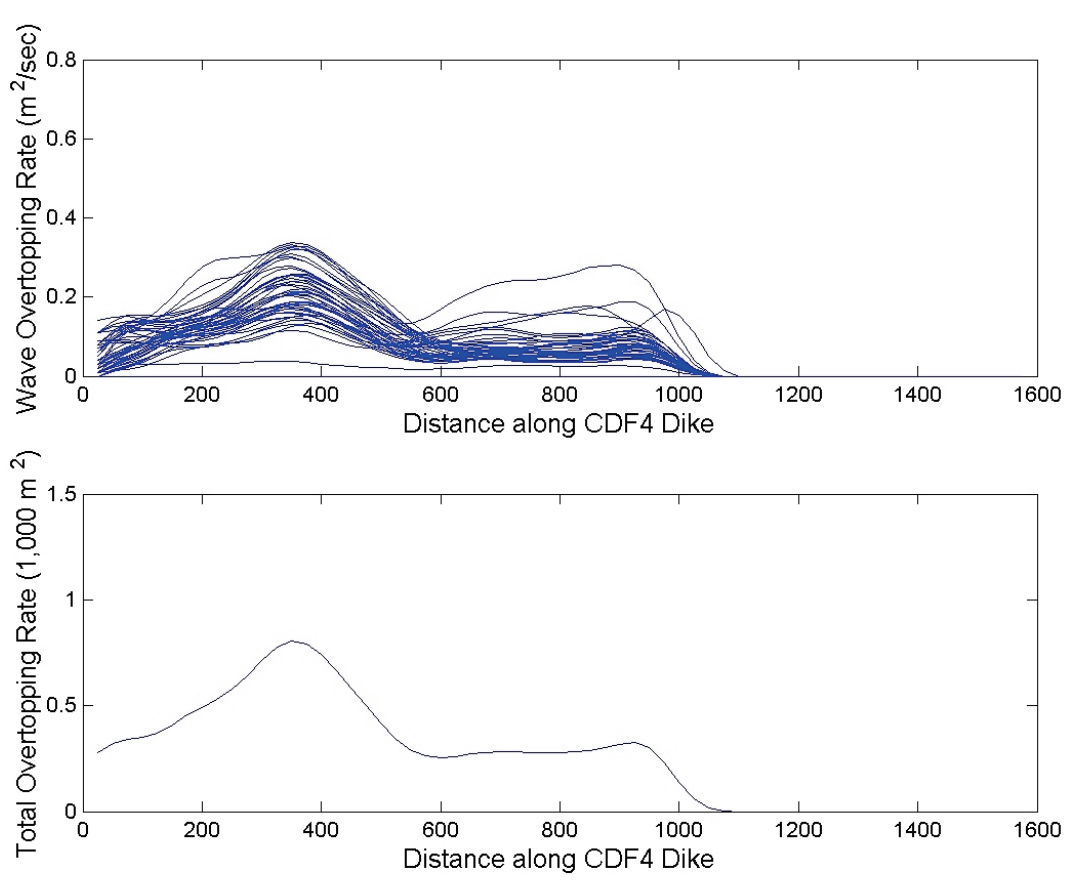
Figure 3-22. Hourly and total wave overtopping rates for impermeable structures (Storm Condition 3).

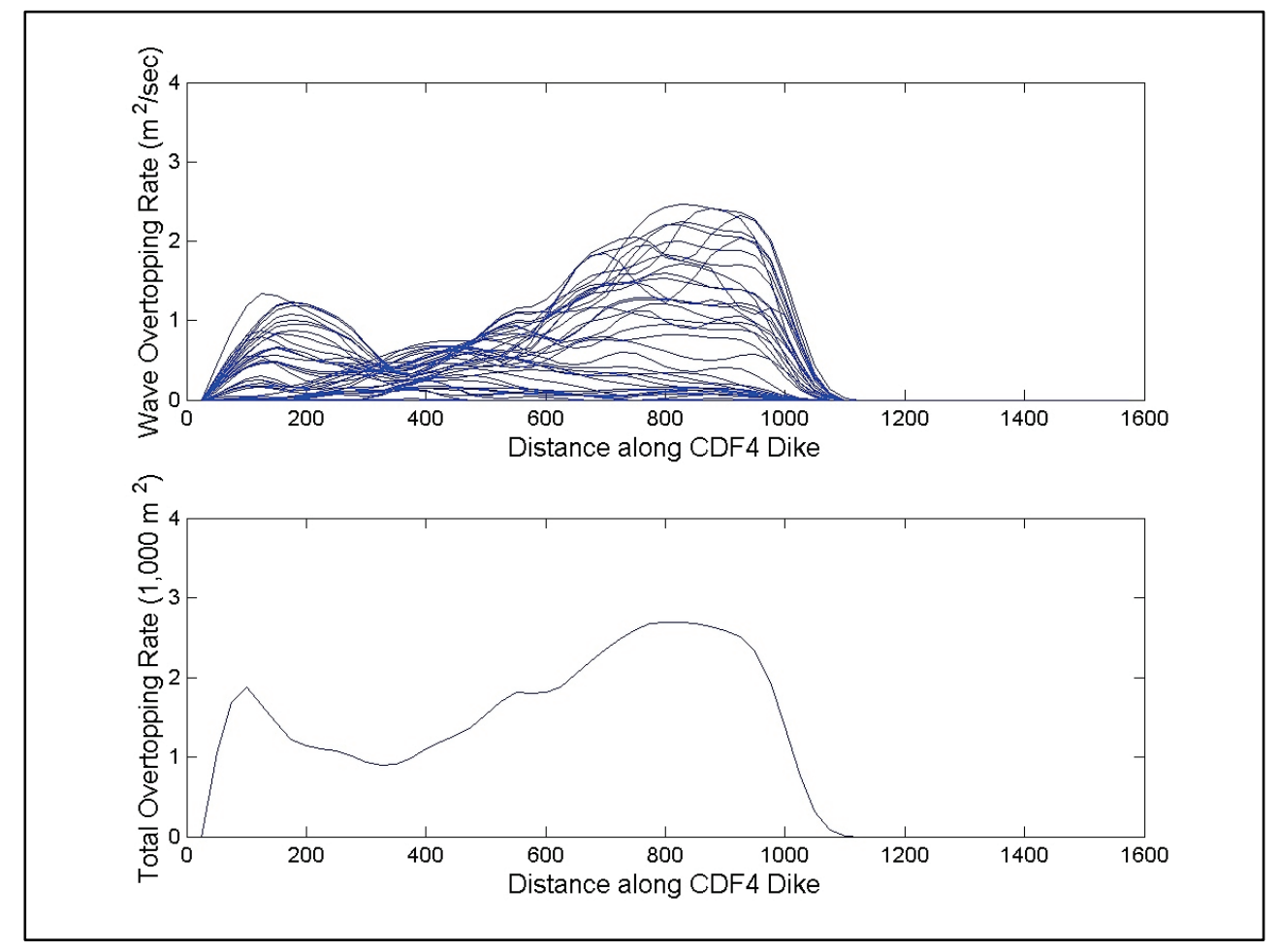

\subsection{Discussion of results}

CMS-Wave modeling results for maximum wave overtopping are listed in Tables 3-1, 3-2 and 3-3. From three storms, the maximum rate of wave overtopping calculated for the impermeable structure is overall much greater than the permeable structure condition because of increased water levels and wave runup in front of and over the structure. The maximum overtopping rate per unit width for the impermeable dike structure ranges from 22.6 to $33.4 \mathrm{ft}^{2} / \mathrm{sec}\left(2.1\right.$ to $3.1 \mathrm{~m}^{2} / \mathrm{sec}$ ). For the permeable dike structure, the maximum overtopping rate varies from 3.6 to $6.0 \mathrm{ft}^{2} / \mathrm{sec}$ (0.33 to 0.56 $\mathrm{m}^{2} / \mathrm{sec}$ ) and with increased overtopping rate for the Storm Condition 2 that has a larger wave and higher storm surge. Model simulations show the highest total overtopping volume associated with the permeable dike. The total storm water volume increase inside $\mathrm{CDF}_{4}$ for the permeable dike ranged from 536,000 to $1,024,000 \mathrm{yd}^{3}$ (410,000 to $783,000 \mathrm{~m} 3$ ). For the impermeable structure, the calculated total volume increase inside $\mathrm{CDF}_{4}$ is approximately 798,000 yd3 $\left(610,000 \mathrm{~m}^{3}\right)$ for the Storm Conditions 1 and 2. Depending on the pre-storm water level inside $\mathrm{CDF}_{4}$, a large storm water volume increase inside $\mathrm{CDF} 4$ can cause potential overflow over the dike to the back corner and along channel side of the CDF4. This could become more severe with the smaller $\mathrm{CDF}_{4}$ fill capacity in future scenarios. 
Table 3-1. Maximum wave overtopping rate and total volume (Storm Condition 1).

\begin{tabular}{|l|c|c|}
\hline Dike Property & Maximum Rate $\left(\mathrm{m}^{2} / \mathrm{sec}\right)$ & Total Volume $\left(\mathrm{m}^{3}\right)$ \\
\hline Permeable structure* & 0.38 & 767,000 \\
\hline Permeable dike* & 0.44 & 783,000 \\
\hline Impermeable structure & 2.17 & 606,000 \\
\hline
\end{tabular}

* assumed low permeable dike layer above the LWD

Table 3-2. Maximum wave overtopping rate and total volume (Storm Condition 2).

\begin{tabular}{|l|c|c|}
\hline Dike Property & Maximum Rate $\left(\mathrm{m}^{2} / \mathrm{sec}\right)$ & Total Volume $\left(\mathrm{m}^{3}\right)$ \\
\hline Permeable structure* & 0.42 & 229,000 \\
\hline Permeable dike* & 0.56 & 705,000 \\
\hline Impermeable structure & 3.10 & 612,000 \\
\hline
\end{tabular}

* assumed low permeable dike layer above the LWD

Table 3-3. Maximum wave overtopping rate and total volume (Storm Condition 3).

\begin{tabular}{|l|c|c|}
\hline Dike Property & Maximum Rate $\left(\mathrm{m}^{2} / \mathrm{sec}\right)$ & Total Volume $\left(\mathrm{m}^{3}\right)$ \\
\hline Permeable Structure* & 0.33 & 400,000 \\
\hline Permeable Dike* & 0.33 & 410,000 \\
\hline Impermeable Structure & 2.47 & 174,000 \\
\hline
\end{tabular}

* assumed low permeable dike layer above the LWD

Estimates of wave overtopping by the CMS were compared to the Eurotop manual, which has become international standard for wave runup and overtopping calculations for simple structure configurations. The following Eurotop formula for impermeable structure was used:

$$
q_{w, \max }=0.2 \sqrt{g H_{s}^{3}} \exp \left(-2.6 \frac{R_{c}}{H_{s}} \frac{1}{\gamma_{r} \gamma_{\beta}}\right)
$$

where $R_{c}$ is defined as the crest freeboard or the crest height of a structure relative to the mean water level. For rubble-mound structures, the crest freeboard is the top of a crest element but not necessarily the height of the rubble mound armor. With this definition and using values of the crest elevation $R_{c}=2 \mathrm{~m}$ and 1 for both gamma parameters, the overtopping rate of $1.56 \mathrm{~m}^{2} / \mathrm{sec}$ was obtained for Storm Condition 1. For Storm Conditions 2 and 3 with $R_{c}=1.3 \mathrm{~m}$ and $R_{c}=1.6 \mathrm{~m}$, the calculated overtopping rates were 
$3.25 \mathrm{~m}^{2} / \mathrm{sec}$ and $2.12 \mathrm{~m}^{2} / \mathrm{sec}$, respectively. In these estimates, the $4.2 \mathrm{~m}$ average crest elevation of dike for Legs 4 and 5 was used.

Comparison of these Eurotop estimates and maximum rates calculated by the CMS for impermeable structure (Tables 3-1, 3-2, and 3-3) show good agreement regardless of Eurotop estimate is for idealized structure geometry while the CMS calculation is for the CDF configuration. For Storm Condition 2, the maximum overtopping rates calculated by the CMS and by the Eurotop formula are 3.10 and $3.25 \mathrm{~m}^{2} / \mathrm{sec}$, respectively. For Storm Condition 3, the rates are 2.47 and $2.12 \mathrm{~m}^{2} / \mathrm{sec}$, respectively.

CMS-Wave results provided in Figures 3-15 through 3-22 show details of the spatial variation in the hourly and total wave overtopping rates occurring along permeable and impermeable dike structure types. 


\section{Boussinesq Wave Modeling}

\subsection{Modeling approach}

Boussinesq wave modeling details for the $\mathrm{CDF}_{4}$ are described in this chapter. This modeling was performed for the existing $\mathrm{CDF}_{4}$ system, two future fill scenarios of the $\mathrm{CDF}_{4}$ interior (Future Fill 2025 and Future Fill 2036), and a raised dike configuration.

As noted in Chapter 3, two numerical wave models, CMS-Wave and BOUSS2D (B2D), were used in the present study. CMS-Wave was applied to a large domain, covering deep-water offshore to nearshore areas. The computational efficiency of CMS-Wave permits simulation of many wave conditions to investigate the proposed modifications and future fill conditions at CDF4. When addressing a broad range of wave modeling needs of projects, computational constraints require the use of a combination of spectral and Boussinesq-type wave models. In this study, deep-water waves in Lake Erie were transformed to the project site using CMS-Wave.

B2D was used for the nearshore wave modeling between approximately the $65 \mathrm{ft}(20 \mathrm{~m})$ depth contour and land (shoreline). This model is appropriate for smaller domains with a limited number of wave conditions. B2D is capable of modeling linear and nonlinear nearshore wave processes in intermediate to shallow water depths. The model handles both short- and long-period waves by solving time-domain, shallow-water nonlinear wave equations. Additional information about B2D is available from references and recent applications (Demirbilek et al. 2016, 2015a, 2015b, 2009, 2008, 2007a, 2008, 2009; Nwogu and Demirbilek 2001, 2006, 2008, 2010;

Nwogu 2007, 2009). Although B2D can address a broad range of nearshore wave processes in coastal projects, potentially long computational times can limit the extent of model domains and number of simulations. When dealing with large domains and many conditions or alternatives, it may be necessary to use B2D with a spectral model. Lin and Demirbilek $(2012,2005)$ provide details of a coupled B2D and CMS-Wave modeling approach for coastal applications.

B2D simulations for three storms and associated water levels were conducted for the existing $\mathrm{CDF}_{4}$, two future fill conditions, and a raised dike. Effects of wave diffraction, reflection, refraction, shoaling, breaking, and nonlinear wave-wave and wave-current interactions are included in 
these simulations. B2D provides estimates of wave parameters (height, period, and direction), wave-induced currents (circulation), and infragravity (IG) waves that are known to be a potential source of harbor surge problems (Demirbilek et al. 2005a,b, 2007a,b,c; Nwogu and Demirbilek 20012006; Nwogu 1993, 1994, 2006). This model does not consider wind generation and growth effects because its modeling domains are generally less than $5 \mathrm{~km}$ in length. The wave growth by wind over such short fetches is negligible. The numerical models used in this study could not be calibrated and validated because there were no field data available.

Three primary wave parameters of interest for $\mathrm{CDF}_{4}$ wave modeling and estimates of overtopping are significant wave height, spectral peak period, and mean wave direction. Detailed information about these parameters is available in the "Wave Mechanics" chapter of the CEM (Demirbilek and Vincent 2015). The CEM describes additional key variables including the mean water depth $(h)$, wave crest-to-trough height $(H)$, and wavelength $(L)$, which are related to wave period and define a steady-progressive wave. Three dimensionless quantities $(H / h, L / h$, and $H / L)$ may be used to characterize nonlinear shallow-water waves. In most practical applications, the wave period is given rather than the wavelength. Because waves in a prototype coastal environment often travel on a finite current, the corresponding wave celerity and wave period vary with the background current (i.e., waves travel faster with the current than against it). B2D takes into consideration the nonlinear effect of currents on waves.

The nonlinear features of shallow-water waves in Boussinesq wave theory are defined using two fundamental parameters, $H / L$ and $H / h$, which quantify the dispersion and nonlinearity of waves, respectively. As depth decreases, the wave nonlinearity generally increases with the wave height, leading to an increase in wave asymmetry and eventual breaking and deformation of the wave profile. While not all features of nonlinear shallowwater wave processes can be described accurately by nonlinear wave models, field measurements have shown that Boussinesq-type wave models are capable of capturing the most essential characteristics of nonlinear shallowwater waves in coastal engineering applications (Demirbilek et al. 2016, 2015a,b, 2007b,c; Nwogu and Demirbilek 2001, 2006; Nwogu 2006; Nwogu 1993, 2008). B2D was expected to represent changes in wave characteristics and nonlinear shallow-water wave processes affecting waves outside the CDF4. The model was used for investigating the interaction of waves with 
the curved dike and other protecting structures, including estimating wave runup and overtopping affecting the interior of $\mathrm{CDF}_{4}$.

\subsection{Model grids and configurations investigated}

The interior of $\mathrm{CDF}_{4}$ is protected by the Perimeter Dike from the incident storm waves on Lake Erie. The lake area exterior to the $\mathrm{CDF}_{4}$ is the primary source of waves that overtop the dike and inundate the interior area of the $\mathrm{CDF} 4$. A small lakeside area near the curved dike is included in the modeling domain by extending model grids to approximately the $36 \mathrm{ft}(11 \mathrm{~m})$ depth contour. The modeling domain covered the exterior and interior of $\mathrm{CDF}_{4}$ areas, the SEAB, SPB, entrance channel that leads into the interior harbor, the detached breakwater north of the entrance channel that protects the harbor (South Breakwater), and adjacent shorelines. As shown in Figures 4-1 through 4-5, parts of Buffalo Harbor were included in B2D grid without the details of the harbor's existing infrastructure.

The model grid lakeside boundary of $\mathrm{B} 2 \mathrm{D}$ is referred to as the "wavemaker" boundary. Details of the bathymetry in the $\mathrm{CDF}_{4}$ exterior and interior, shorelines, structures, Buffalo Harbor, entrance channel, and other prominent features are included in the grid. Only one B2D grid was needed for simulations because wave period and wave direction were essentially the same for the three conditions (Table 4-1). The baseline grid developed initially for the existing $\mathrm{CDF}_{4}$ was modified for two future fill scenarios and a raised Perimeter Dike by incorporating interior elevations of $\mathrm{CDF}_{4}$ for scenarios Future Fill 2025 and Future Fill 2036 and by raising the dike's crest elevation. As shown in Chapter 3, recent survey data indicated the crest elevation of the curved dike varied along the dike. Figures 4-1(a) and 4-1(b) show one randomly drawn transect (red line) and profile (elevation) along this transect. Figure 4-1(c) is a distorted scale sketch of the dike structure cross section, showing the $+15 \mathrm{ft}(4.6 \mathrm{~m})$ LWD design crest elevation. In Figure 4-1, the $x$-axis is the distance along the transect and the $y$-axis is the structure elevation, and both are in meters. See Figure 2-6 for additional structural details. 
Figure 4-1. Perimeter Dike with (a) an arbitrary transect (red line), (b) elevation along thetransect (vertical and horizontal dimensions are in meters), and (c) design cross section of dike structure (vertically exaggerated).
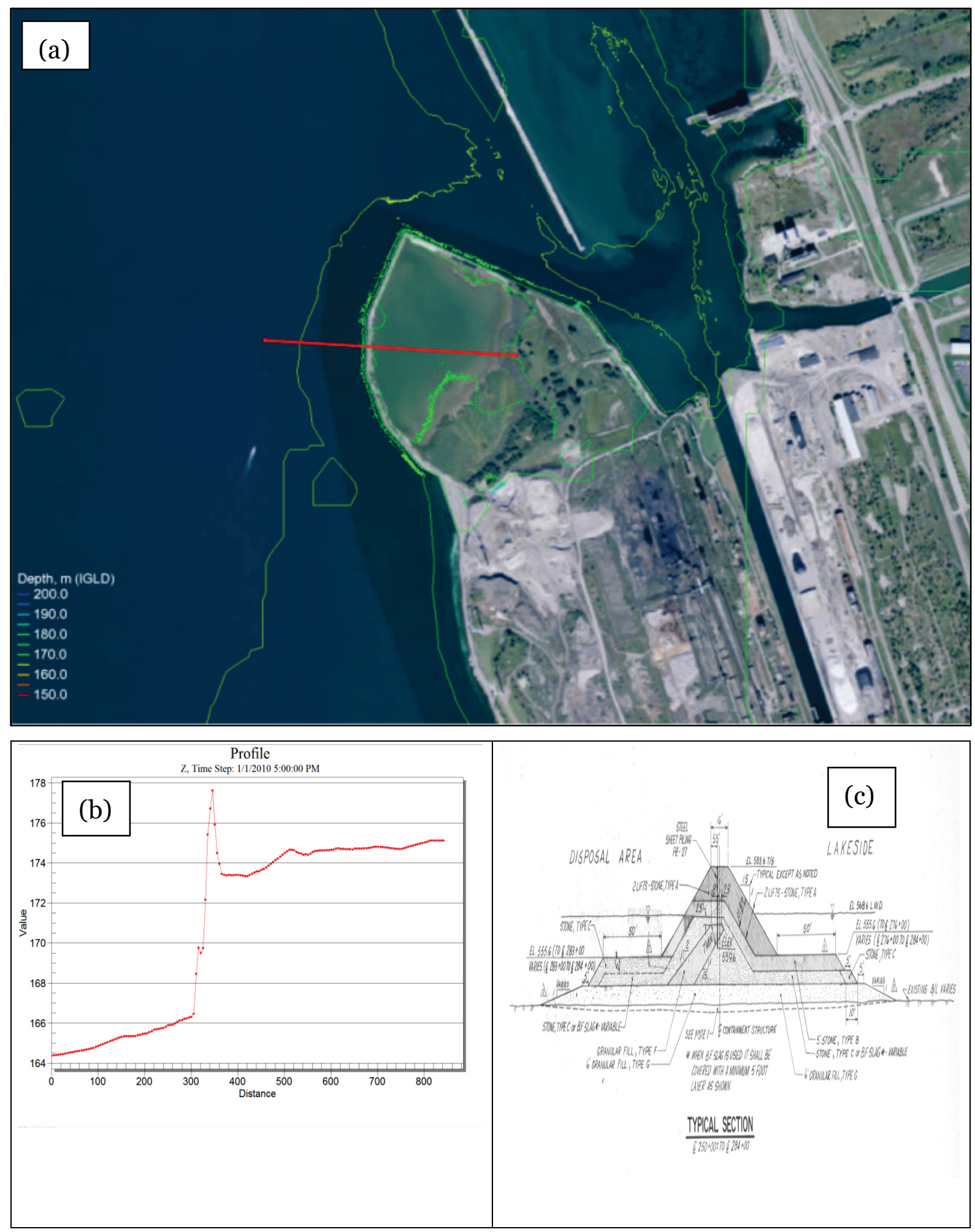
The dike elevation in Figure 4-1(b) may be calculated by subtracting the low water lake datum (173.5 m, LWD) from the values in the figure. For this transect, the dike crest elevation is approximately $+4 \mathrm{~m}$. Additional transects passing through different segments (legs) of the Perimeter Dike indicated that the dike crest elevation varied from south to north, confirming that dike's elevation was not constant. Recent survey data by LRB indicated the crest elevation along the Perimeter Dike can be several feet (nearly $1 \mathrm{~m}$ ) below the design elevation of $15 \mathrm{ft}(4.6 \mathrm{~m})$. Chapter 2 provides additional information about the condition of structures protecting the $\mathrm{CDF} 4$ interior.

The B2D model grid for the existing $\mathrm{CDF}_{4}$ (Figure 4-2) covers a rectangular area of 1.55 miles $\times 1.12$ miles $(2.5 \mathrm{~km} \times 1.8 \mathrm{~km})$ with a constant cell size of $13.1 \mathrm{ft}(4 \mathrm{~m})$.

Figure 4-2. B2D grid for existing CDF4 overlaid on a Google map image (origin of grid is at lower left corner).

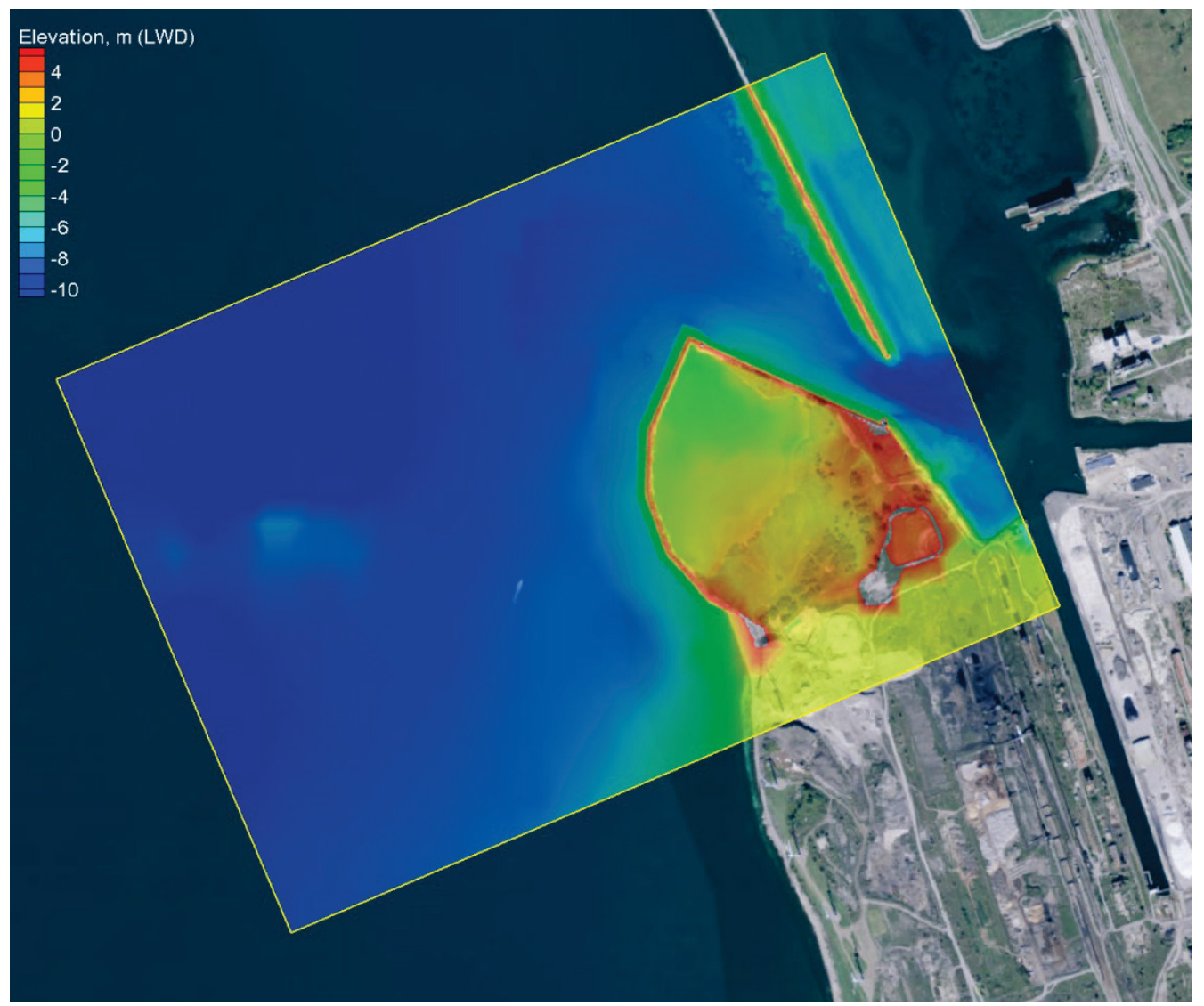


The CDF4 structures are composite systems consisting of stones, sheet piles, and concrete pieces. Segments of protective structures have randomly spaced gaps and ridges, and with a discontinuous crest elevation. Parts of structures have front and back faces with steep slopes. The blue and brown colors in Figure 4-3 represent the water and land areas contained in the grid. Figures 4-4 and 4-5 show a close-up of the CDF4. The vertical dimension is magnified to show details of the exterior, interior, and irregularities of the surrounding structures making up the $\mathrm{CDF} 4$ system. The irregularities of the structures and $\mathrm{CDF}_{4}$ interior area were expected to pose serious computational challenges in B2D modeling.

Figure 4-3. Land (brown) and water (blue) areas in the B2D grid domain.

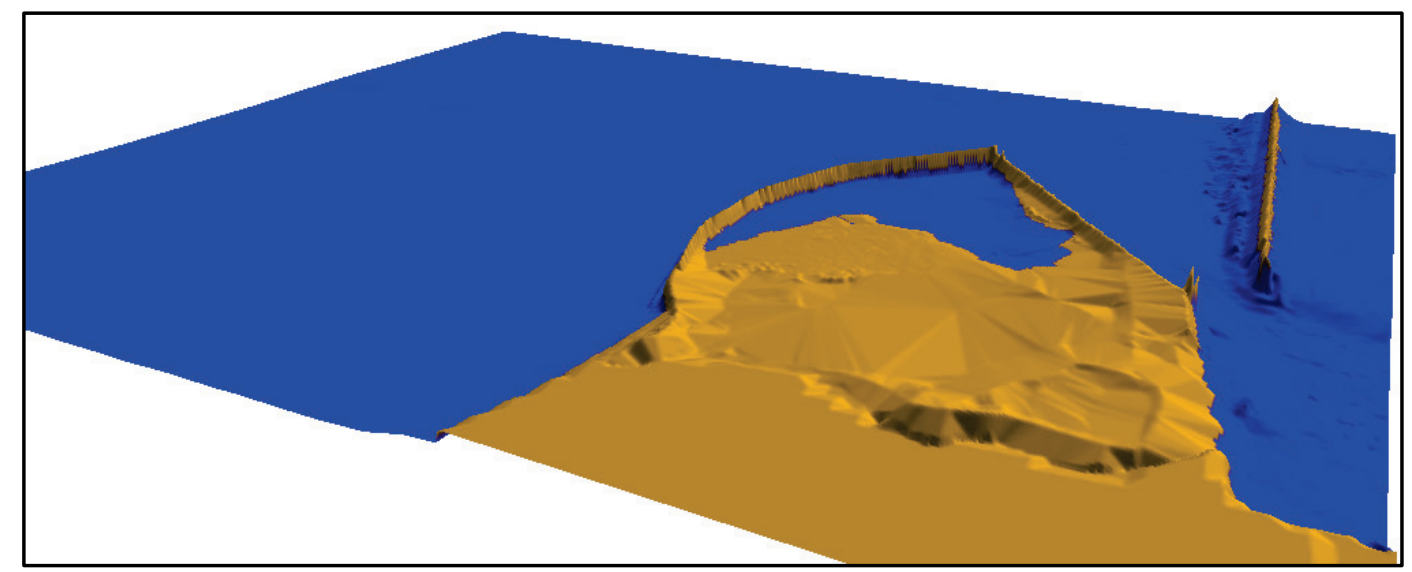

Figures 4-2 through 4-5 provide some details of B2D grid for the existing $\mathrm{CDF} 4$. The origin of the grid is at the lower left corner of the grid domain in Figure 4-2, and the grid is oriented 23 deg counterclockwise with respect to east. The offshore wave boundary of the grid was placed at approximately the $33 \mathrm{ft}(10 \mathrm{~m})$ depth contour. The model grid boundary in the lake is where the incident forcing is applied, so it is also known as the "wavemaker" boundary. The existing $\mathrm{CDF}_{4}$ (without project) grid was also used for the alternatives (with project) by simply modifying the bathymetry/permeability of the CDF4 within the model domain. Following the description of the existing harbor grid, grids for two future scenarios and a raised dike are presented. The same output stations and transects were used for extraction and comparison of modeling results for all geometries investigated. 
Figure 4-4. Close-up of the CDF4 exterior area (vertical coordinate magnified).

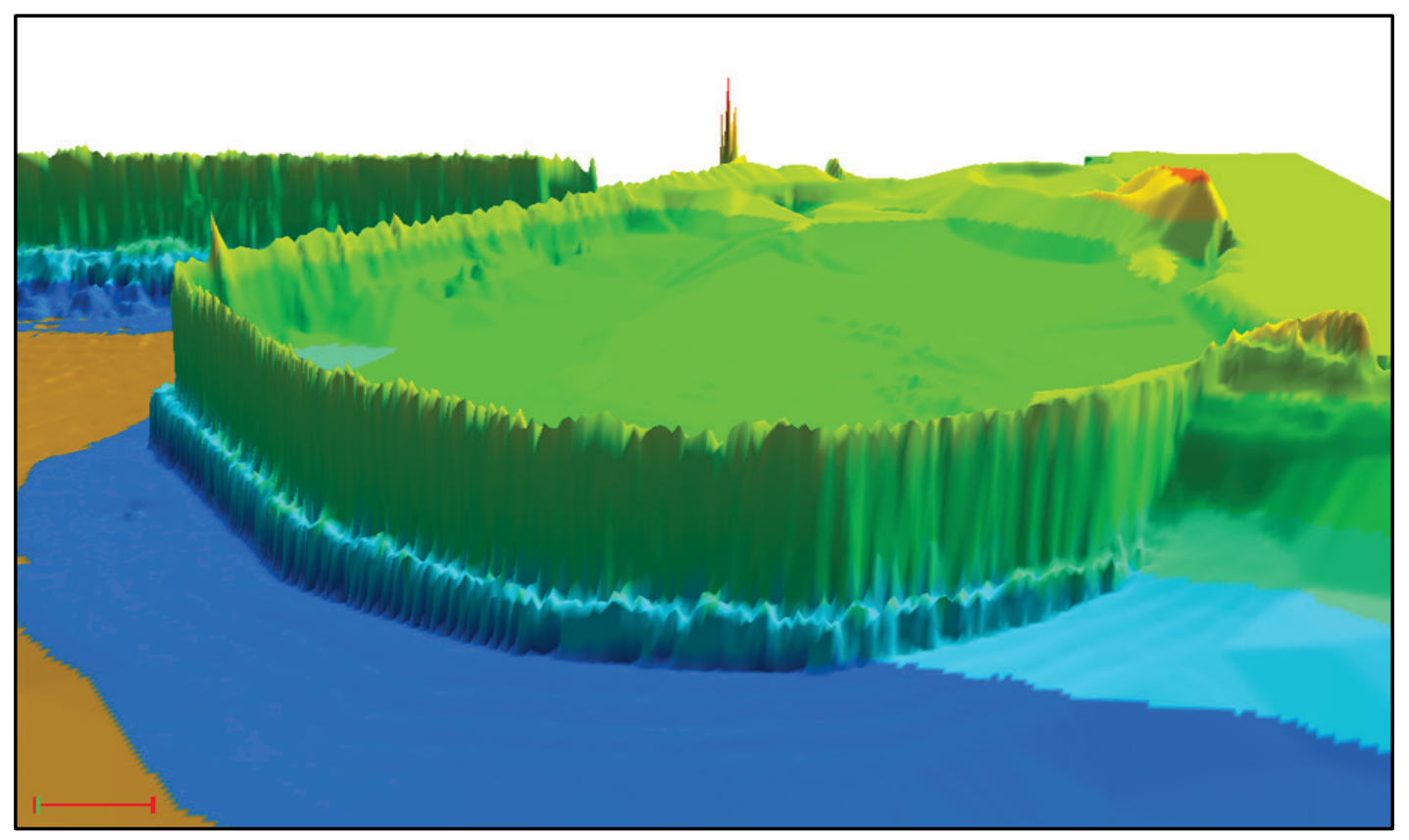

Figure 4-5. Close-up of the CDF4 interior (vertical coordinate magnified).

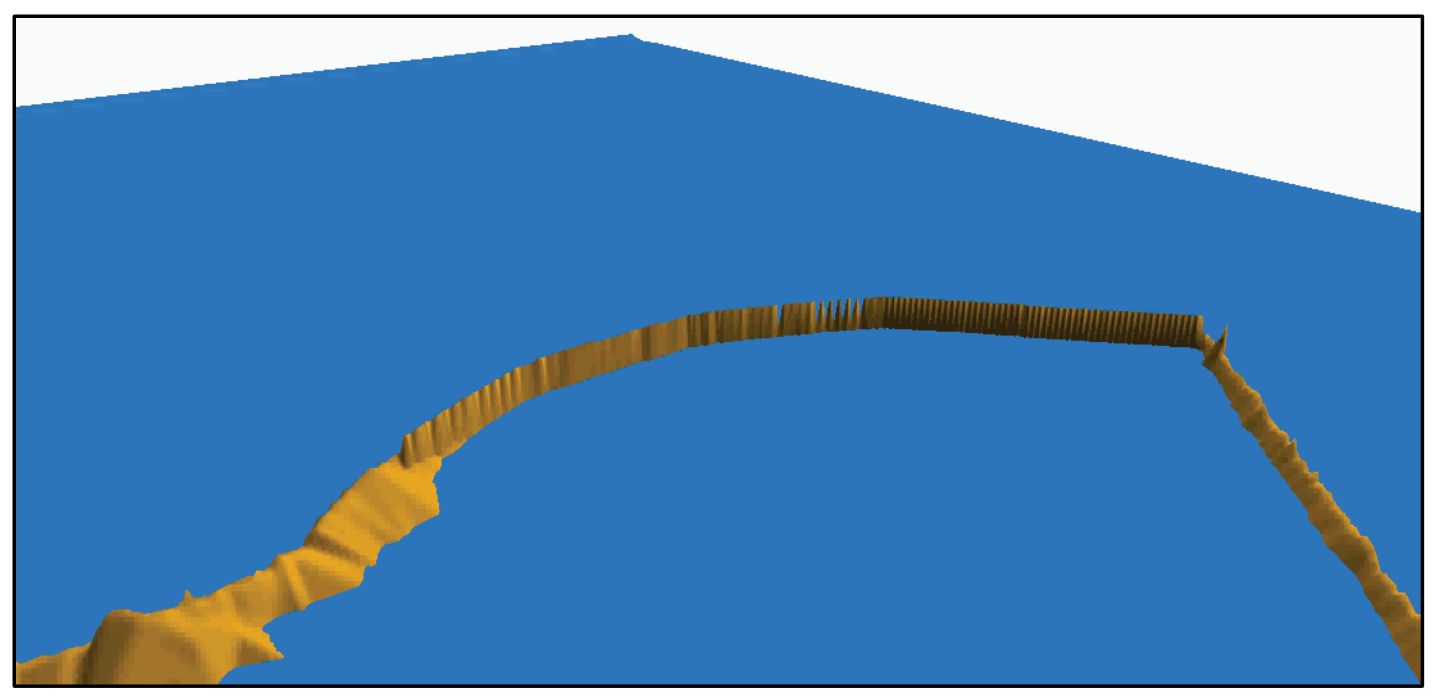

Figure 4-6 and Figure 4-7 are two recent photos showing close-up details of one section of Legs 2-3 and Legs 4-5 of the Perimeter Dike, respectively. These figures show the size and placement of rocks and sheet piles in the mid-section of the dike. During the initial modeling phase, it became apparent that the irregularities and complexities of the curved dike could not be represented at a required detailed level using a 2D model. It was necessary to use both $1 \mathrm{D}$ and 2D Boussinesq models ( $\mathrm{B} 1 \mathrm{D} / \mathrm{B} 2 \mathrm{D})$. This type of wave modeling approach is required when investigating fluid-structure interaction of the curved structures with irregular sides and tops, and gaps and breaks throughout the structure. 
Figure 4-6. Recent photo showing the dike top section repaired crest along Leg 2 and Leg 3.

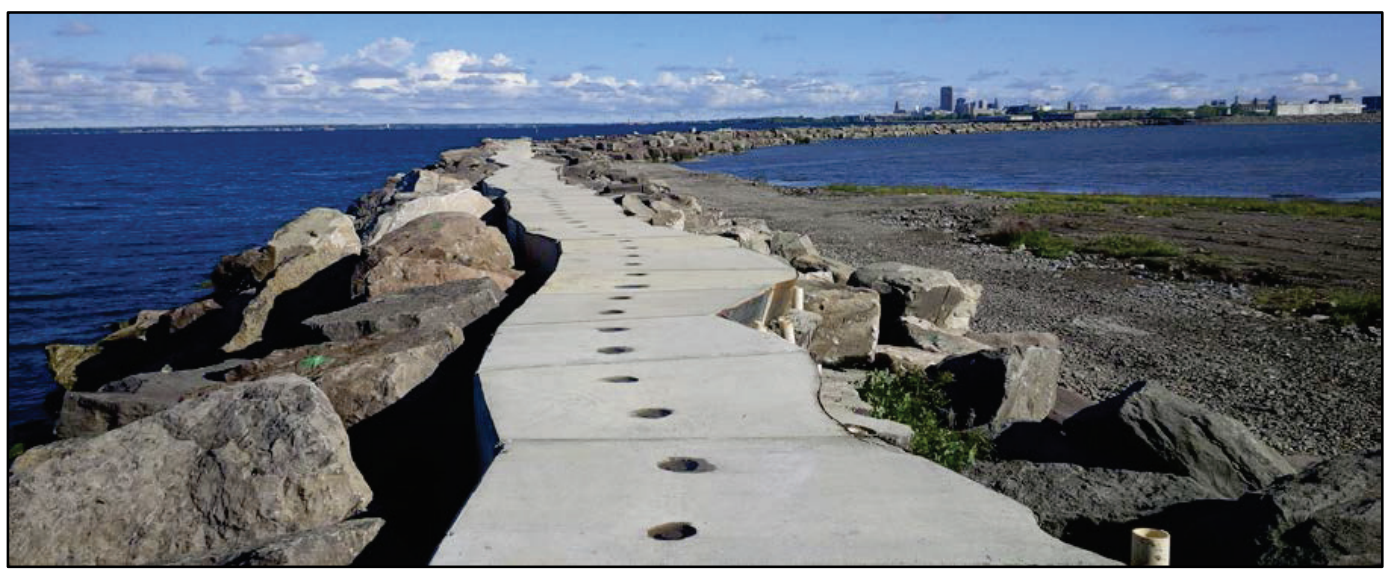

Figure 4-7. Typical view along Leg 4 and Leg 5 in September 2009.

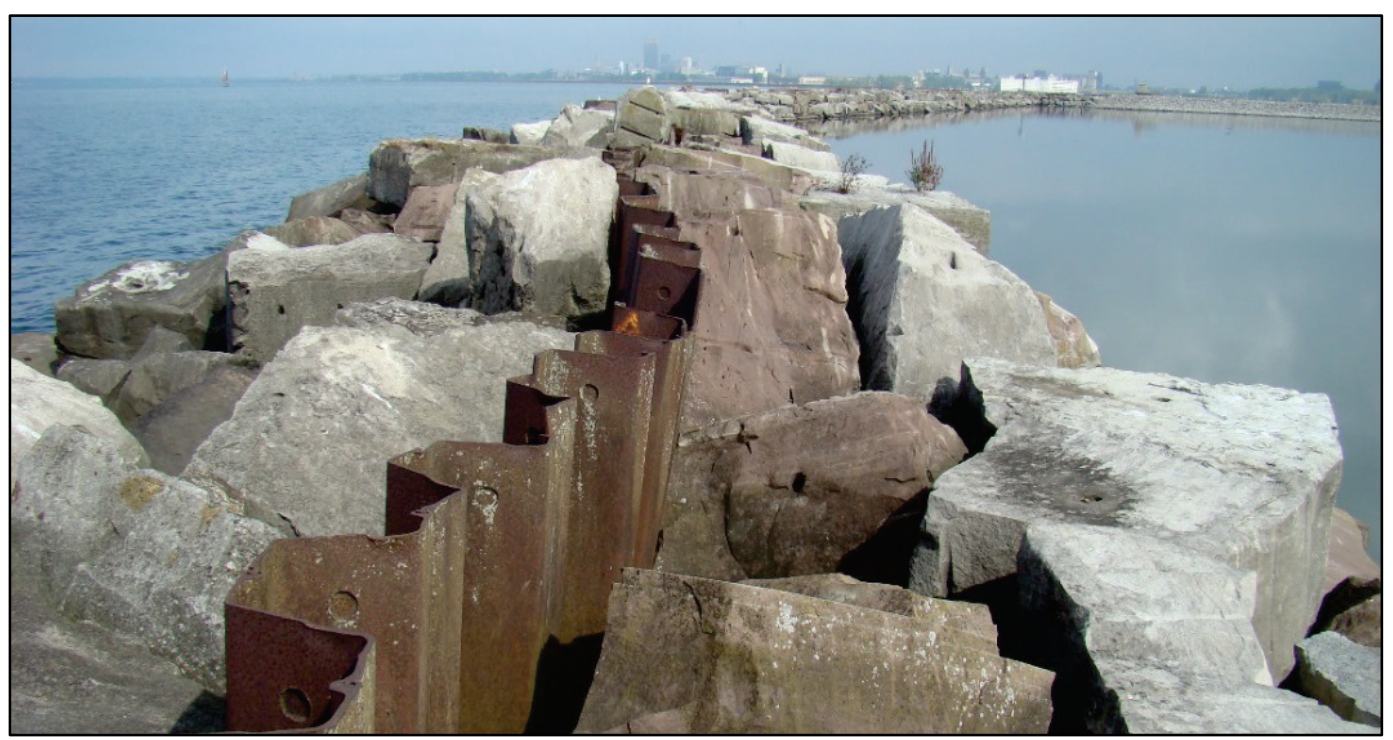

Figure 4-8 shows 14 transects T1 through T14 (also shown in Figure 3-4) used to determine changes occurring in water level and wave parameters across the dike and for calculating wave runup/overtopping across different segments of structures. Transects T1 to T14 are perpendicular to the structural segments, and their lengths vary from $100 \mathrm{~m}$ to $150 \mathrm{~m}$ (330 ft to $490 \mathrm{ft}$ ). See Figures 3-5 to 3-8 for the elevation change along each transect. 
Figure 4-8. Transects T1 through T14 used for runup/overtopping calculations.

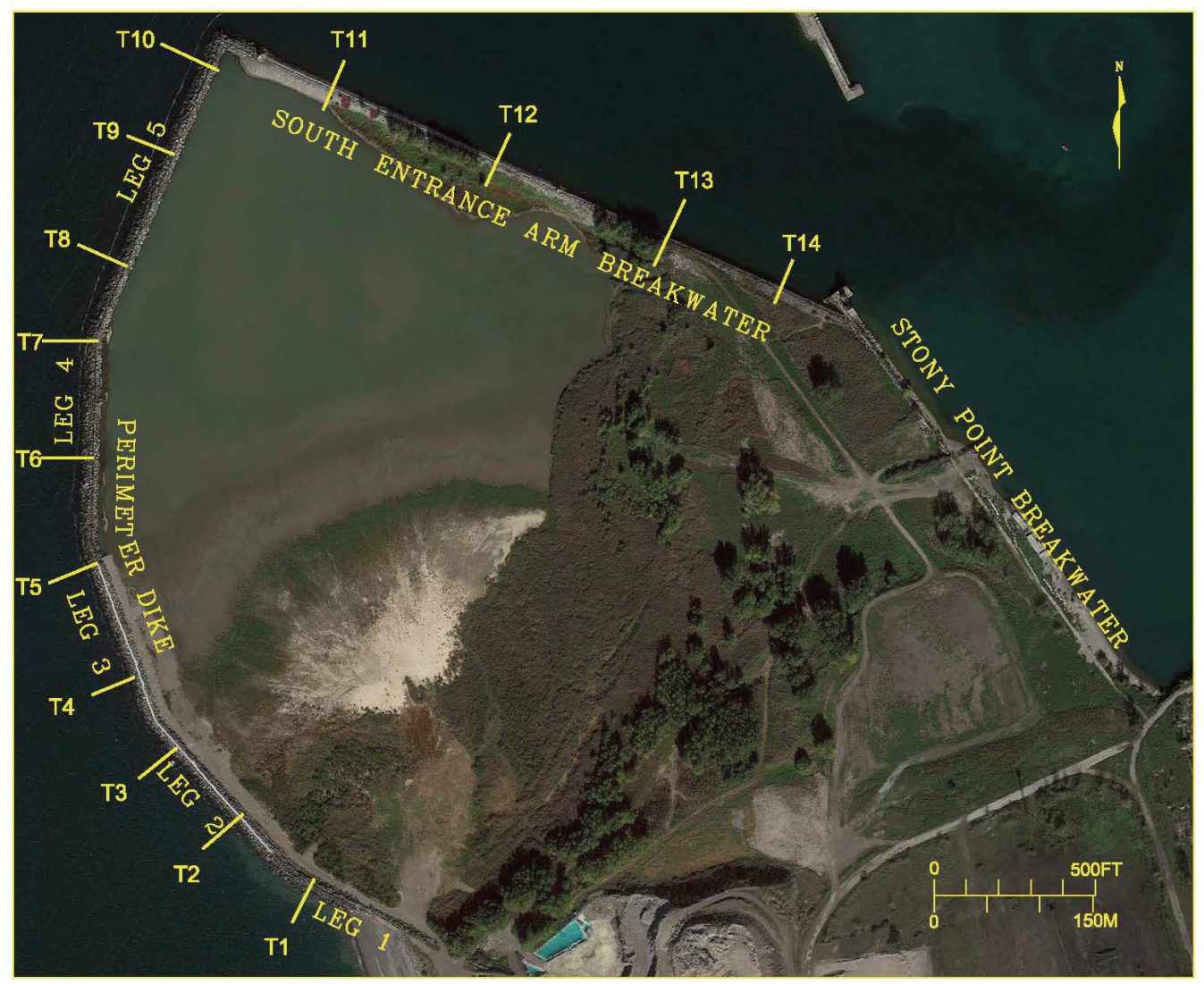

As noted in Chapter 2, two future fill scenarios were modeled in addition to the existing (2015 survey) condition. The future fill scenarios represent condition of the CDF4 interior for Future Fill 2025 and Future Fill 2036. For the Future Fill 2025 condition, the fill elevation was assumed equal to $+8 \mathrm{ft}(\sim+2.4 \mathrm{~m})$ LWD. For the Future Fill 2036 condition, it was assumed that the CDF was filled to elevation $+13 \mathrm{ft}(+4 \mathrm{~m})$ LWD. Figures 4-9 and 410 present the $\mathrm{CDF}_{4}$ interior fill contours for the Future Fill 2025 and Future Fill 2036 scenarios developed by LRB. These interior bathymetries were used to generate the B2D grids for these future scenarios. The final future scenario investigated was a raised dike. The goal was to determine reduction in wave overtopping by raising the crest elevation of the Perimeter Dike by approximately one stone thickness of $5 \mathrm{ft}(1.5 \mathrm{~m})$. For this third future scenario, the gaps in the dike were filled in. This is illustrated in Figures 4-11 and 4-12. 
Figure 4-9. Future year 2025 CDF 4 interior contours.

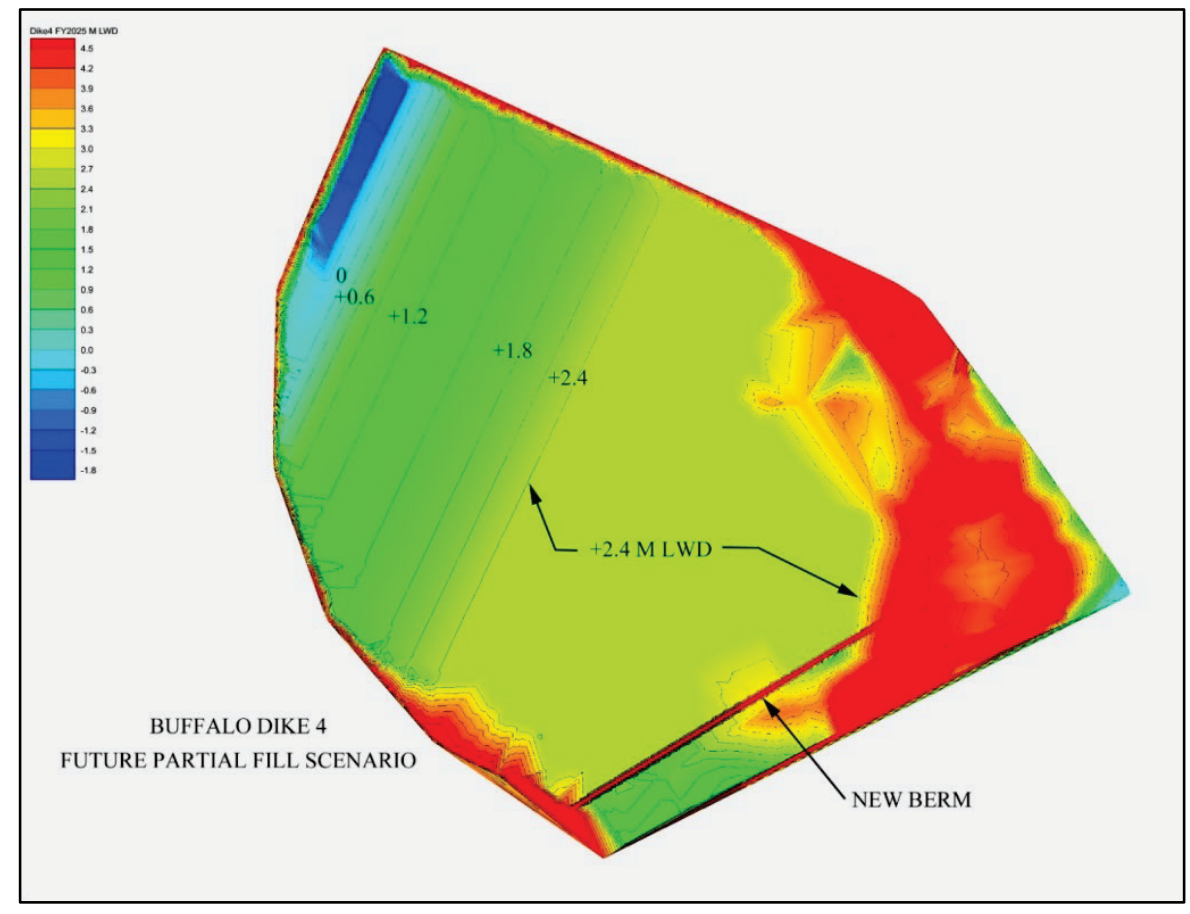

Figure 4-10. Future year 2036 CDF 4 interior contours.

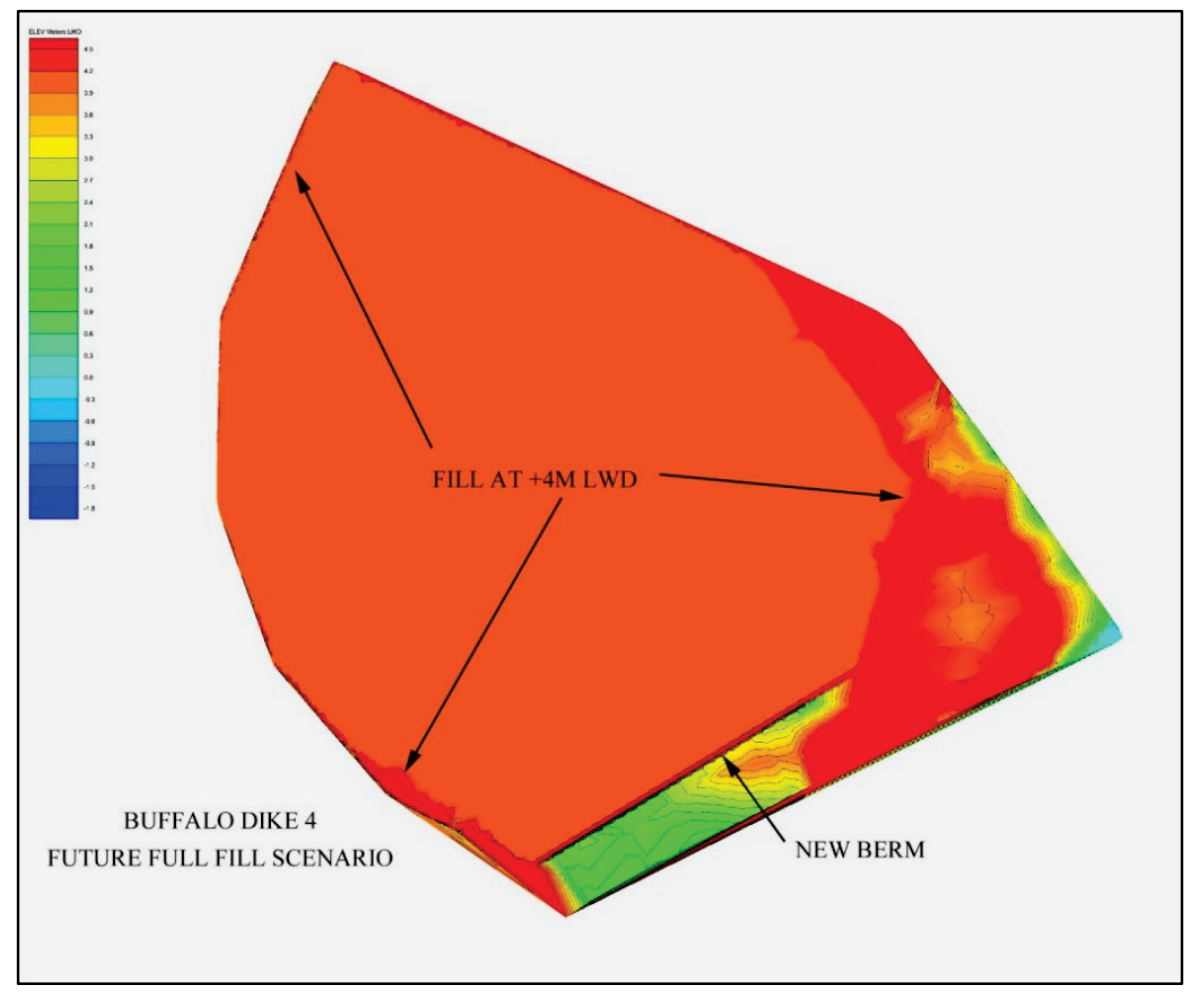


Figure 4-11. Future raised elevation (red color) of Perimeter Dike at $20 \mathrm{ft}(6.2 \mathrm{~m})$.

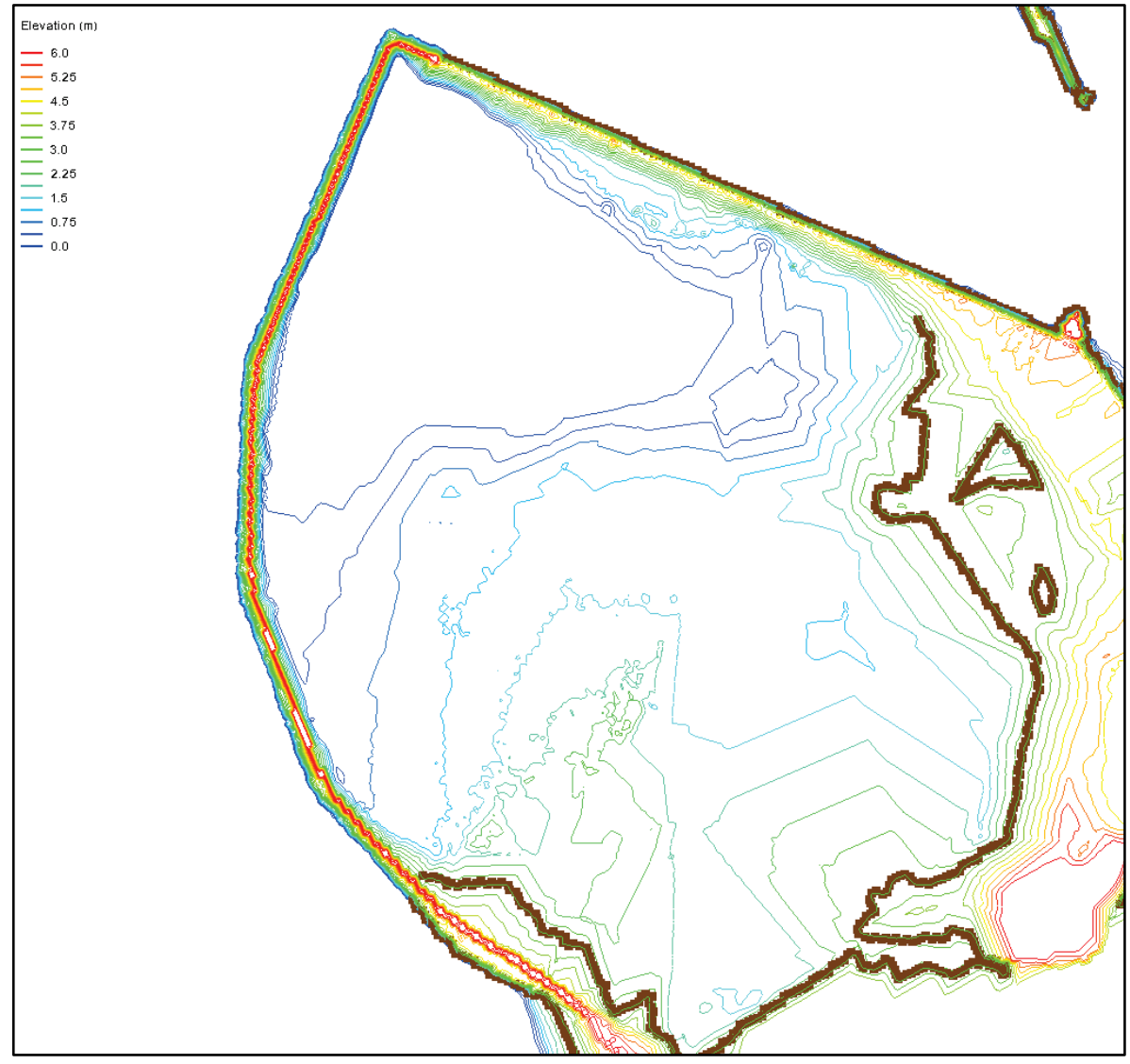

Figure 4-12. Future raised Perimeter Dike without gaps.

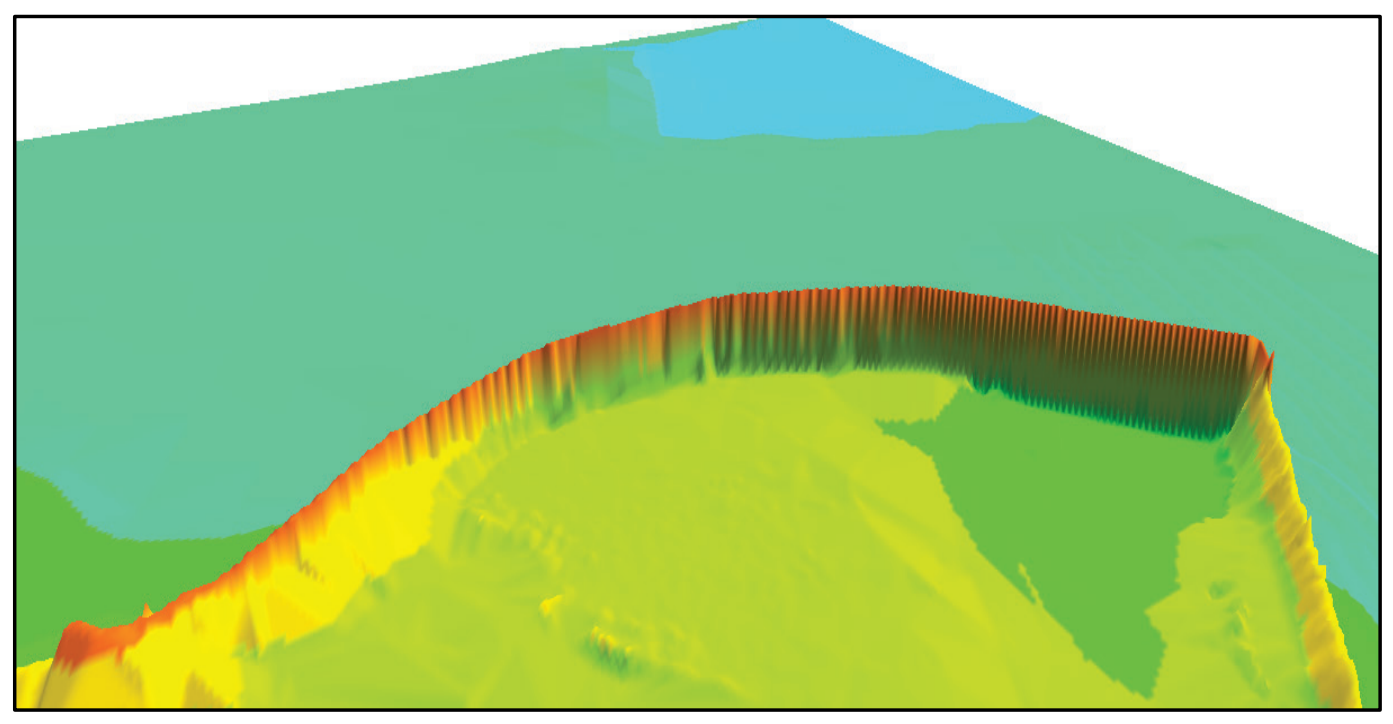




\subsection{Input conditions}

Incident wave inputs for B2D simulations were specified based on directional wave estimates obtained from CMS-Wave modeling as described in Chapters 2 and 3. The deep-water incident wave conditions in Tables 2-6, 2-7, and 2-8 were transformed to the project site with CMS-Wave. Calculated CMS-Wave wave height, period, and direction parameters at the seaward boundary of B2D grids were used in this modeling study. The maximum values of wave heights at the B2D grid boundary were $3.5 \mathrm{~m}, 3.9 \mathrm{~m}$ and $3.6 \mathrm{~m}$ for Table 2-6, 2-7 and 2-8, respectively. For these three transformed conditions, the peak wave period and wave direction were $10 \mathrm{sec}$ and $247 \mathrm{deg}$, respectively. The corresponding water levels were $2.3 \mathrm{~m}, 3 \mathrm{~m}$, and $2.8 \mathrm{~m}$, respectively. Table 4-1 shows input wave parameters and water levels for the three storms used in B2D simulations. The second storm has the largest wave height and water level, but overall these three conditions produced similar forcings at the open boundary (wavemaker) of B2D grid.

Table 4-1. B2D input incident wave parameters and water levels.

\begin{tabular}{|l|c|c|c|c|}
\hline Storm Condition & $\mathrm{H}_{\mathrm{s}}(\mathrm{m})$ & $\mathrm{T}_{\mathrm{p}}(\mathrm{sec})$ & $\theta(\mathrm{deg})$ & $\mathrm{WL}(\mathrm{m})$ \\
\hline $\begin{array}{l}\text { 2-year wave and water level return period } \\
\text { (Storm Condition 1) }\end{array}$ & 3.5 & 10 & 246 & 2.3 \\
\hline $\begin{array}{l}\text { 20-year wave and 10-year water level } \\
\text { return period (Storm Condition 2) }\end{array}$ & 4.0 & 10 & 247 & 3 \\
\hline 1982 storm (Storm Condition 3) & 3.6 & 10 & 248 & 2.8 \\
\hline
\end{tabular}

B2D inputs listed in Table 4-1 indicate large incident waves affecting the study area are mostly from the southwest direction. Waves from the south, west, northwest, and north directions can also reach the project site. However, as described in Chapters 2 and 3 and Appendix C, waves from these directions were not included in design wave conditions mainly because of the shorter fetches associated with these directions. Incident waves directed toward the $\mathrm{CDF}_{4}$ interior are expected to be blocked largely by the Perimeter Dike that protects it on the lake side. Only storm waves that can overtop the dike and those that graze or ride along the dike could possibly inundate the interior of CDF4. Model runs did not show any evidence of wave overtopping from the entrance channel side. Wave heights in the channel were reduced as compared to wave heights along the front face of the dike on the lake side and could not overtop the high elevation of the SEAB. 
Figures 4-13, 4-14, and 4-15 are examples of a B2D model wave height field and two snapshots of the sea surface elevation for an incident wave from the southwest direction. These model results illustrate general features of the wave pattern outside and inside the $\mathrm{CDF}_{4}$ calculated by B2D. Note in Figure 4-13, the focusing of waves on the northern part of the Perimeter Dike along the Legs 3 to 5 . Figures 4-14 and 4-15 display close-up images of the wave surface elevation outside and inside the $\mathrm{CDF} 4$ with two dike sections overtopped. Waves from the lake overtop the front face of structure, some waves break and others reflect and diffract around the structure. Some waves overtop the dike low segments or areas where gaps exist in the structure. Though overtopping the structure, wave energy in the interior of $\mathrm{CDF}_{4}$ is low as compared to the large waves outside. The interior of CDF4 remains relatively calm because there is no wind forcing in $\mathrm{B} 2 \mathrm{D}$ (and the fetch is short as well).

Figure 4-13. An example of overall wave height field.

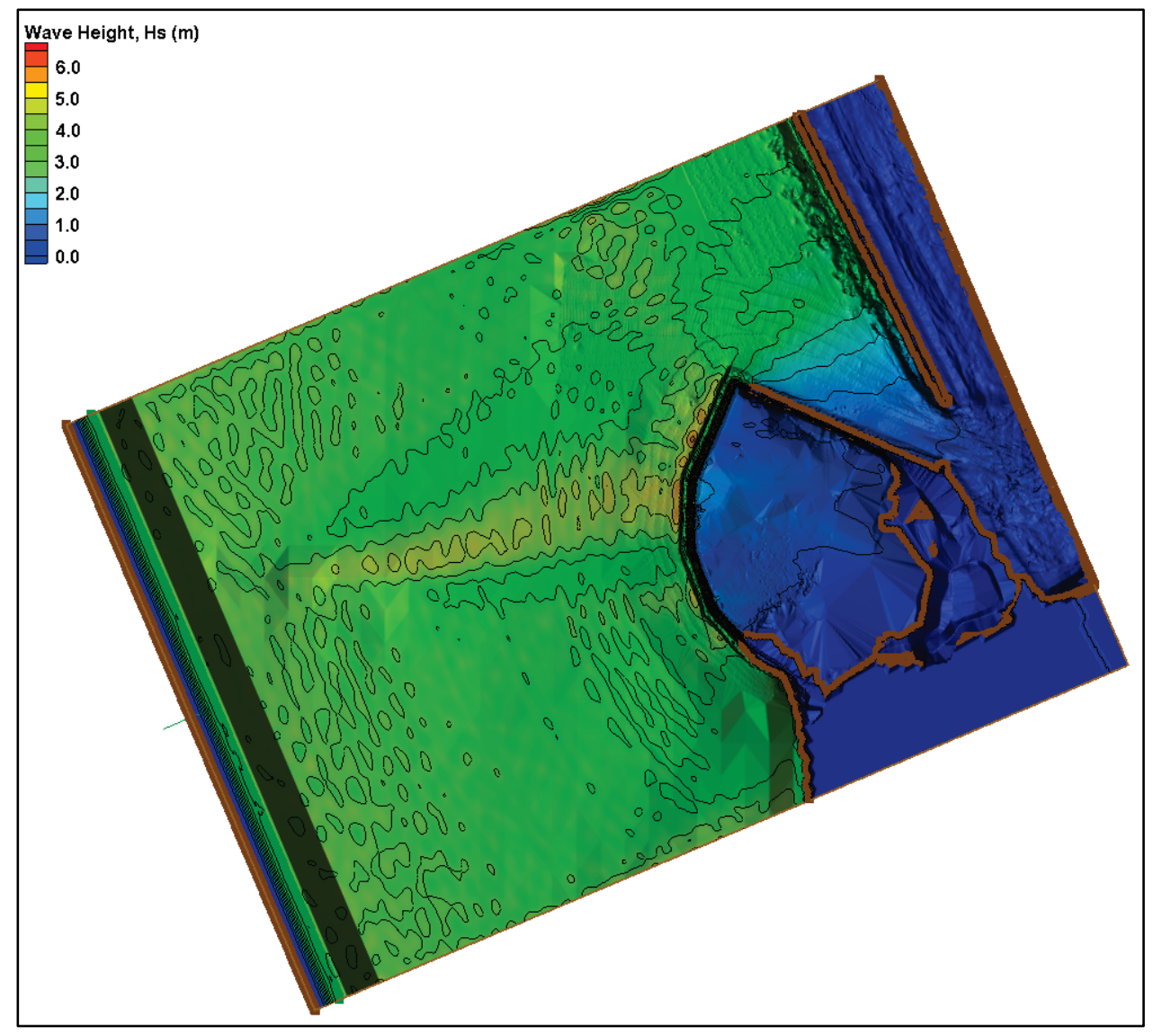


Figure 4-14. View of sea surface elevation in the lake outside the front face of Perimeter Dike.

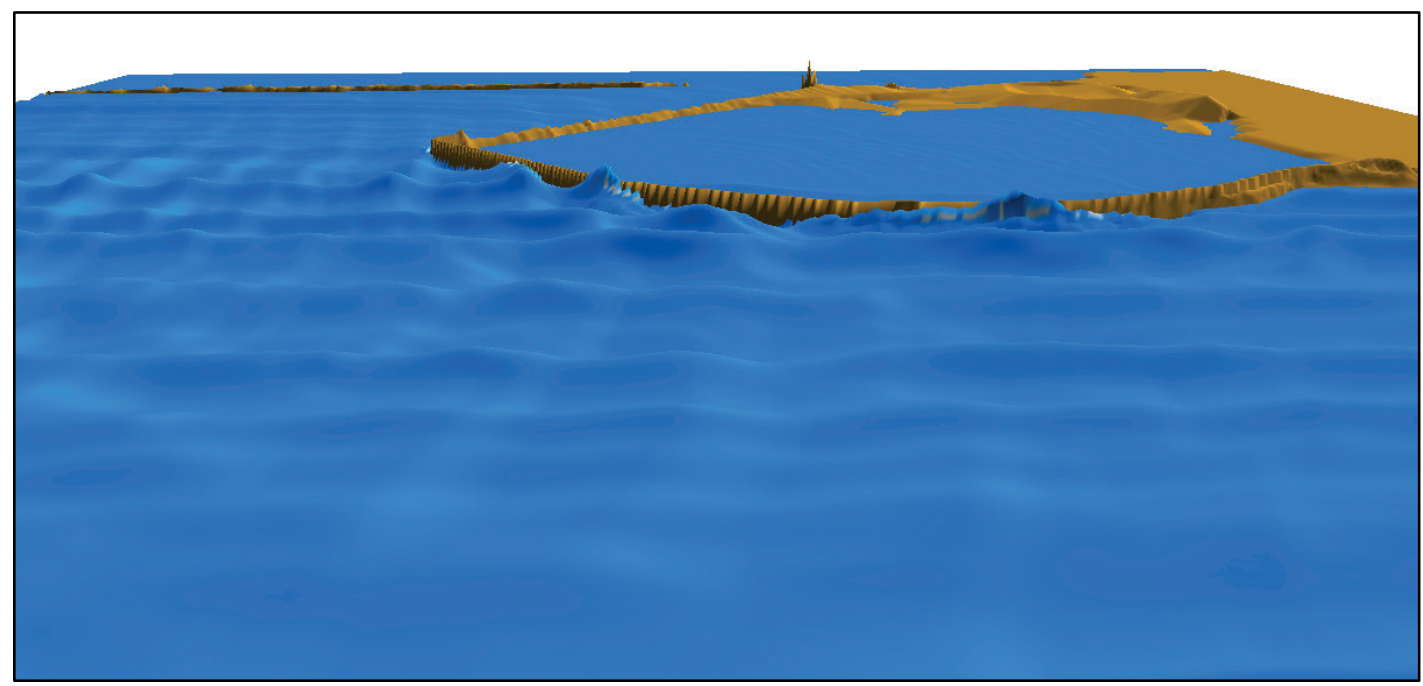

Figure 4-15. View of sea surface elevation from the interior of CDF4.

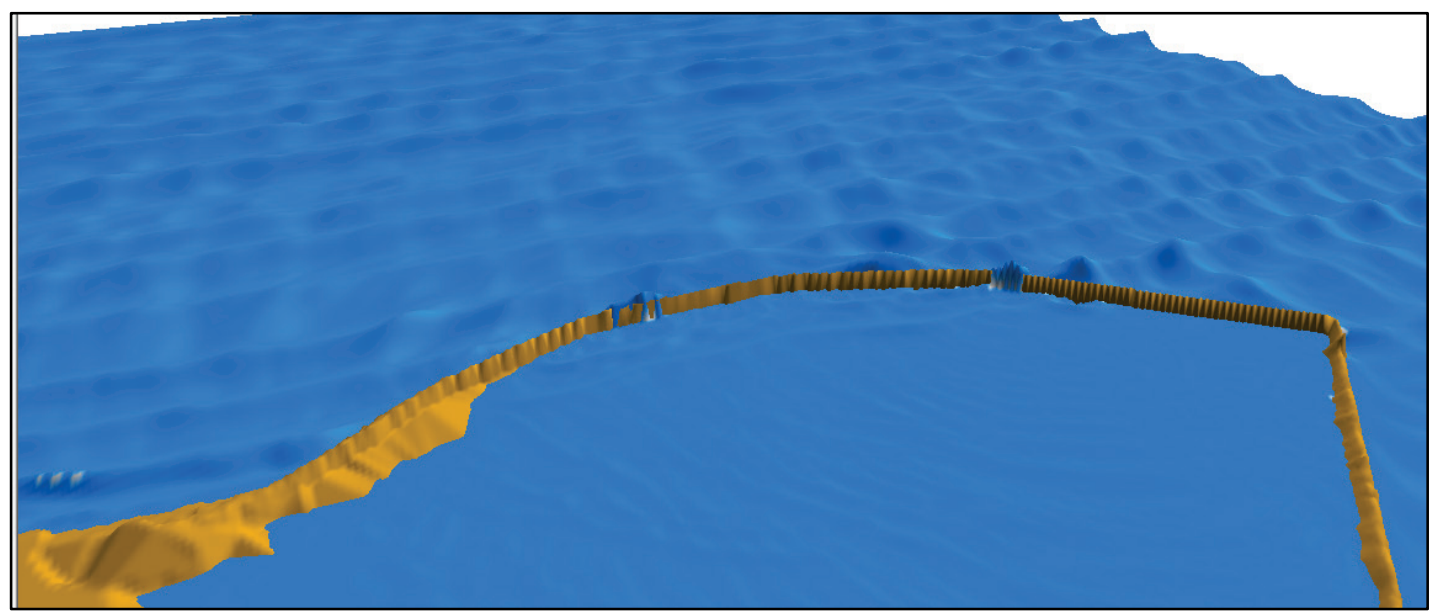

\subsection{Model setup and testing}

An exterior wavemaker was placed along the offshore model grid boundary in the lake. Interior wavemaker locations at constant water depth of $32.8 \mathrm{ft}$ $(10 \mathrm{~m}), 49.2 \mathrm{ft}(15 \mathrm{~m})$, and $65.6 \mathrm{ft}(20 \mathrm{~m})$ were considered to reduce the computational burden, where a constant depth zone at the wavemaker followed a transition zone connecting the rest of the grid domain. Extensive tests conducted for internal and external wave makers indicated the choice of wavemaker had no effect on model results, but an internal wavemaker required a proper selection of the wavemaker location and a transition zone shoreward. An exterior wavemaker was used in the production runs for selected wave conditions for improved computational stability of the model. 
A series of sensitivity tests were conducted for input parameters which influence model solution and numerical stability. These included the Chezy (bottom friction) coefficient, Smagorinsky (turbulence) coefficient, and the damping layer characteristics (width and strength of damping or sponge layers) used to represent the reflectivity of structures and land boundaries. Tests showed the numerical stability of computations was sensitive to the characteristics of the damping layers used in simulations, including their location, width, and coefficient. Partially absorbing, lateral damping layers 16.4 to $82 \mathrm{ft}$ ( 5 to $25 \mathrm{~m}$ ) wide along parts of the lateral grid boundaries were tested.

Damping layers were also placed along the interior and exterior faces of structures in the initial tests. No dampers were used along the lakeside face (front) of the Perimeter Dike in the simulations requiring the computation of wave runup/overtopping. Light damping layers applied along the interior land-water boundaries of the $\mathrm{CDF} 4$ generally improved the numerical stability. Aside from one damping layer used at the down-wave boundary along the Buffalo Harbor shoreline, no damping layers were used in the final production runs. As an example, Figure 4-16 displays the damping layers used in a test run with an internal wavemaker. The damping layers are indicated by brown lines along the grid boundaries and also around the structures.

Test runs were performed to determine proper values of damping layers affecting interaction of waves along the land and structure boundaries inside and outside the $\mathrm{CDF} 4$ system. This testing was necessary in the absence of field data available to calibrate and validate the model. Analysis of test results and animations revealed a weak reflection from mild sloping land, shorelines, and structures. Absorbing or low reflection damping helped to improve the computational stability. A weaker wave reflection from the interior land boundaries of the CDF4 with mild slopes was observed as compared to stronger reflection occurring from some sections of structures. This trend was consistent over a wide range of wave parameters tested. The model stability improved with no damping layers placed on the front face (lakeside) of Perimeter Dike and with dampers in the interior of CDF4. A damping layer was placed on the down-wave boundary along the Buffalo Harbor land boundary to absorb waves reflecting back from the boundary. These findings were applied to final production runs of four configurations investigated. 
Figure 4-16. Example of damping layers used in a test run.

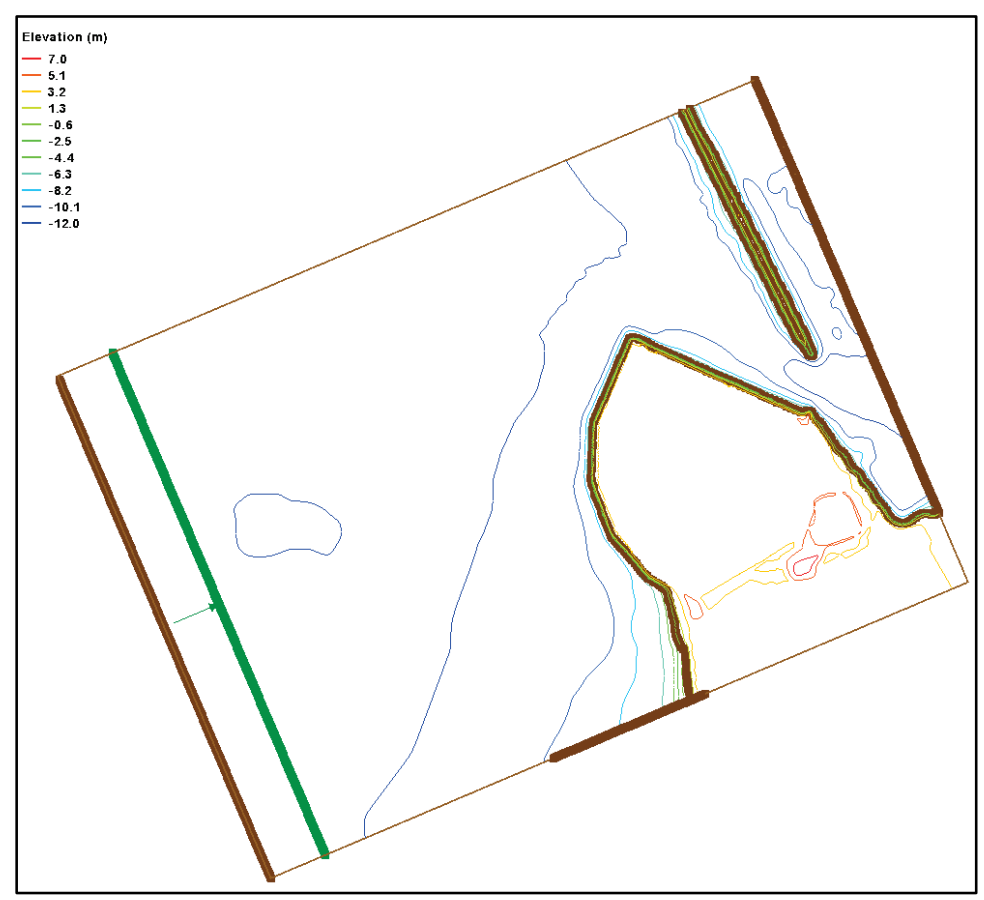

Except inside the $\mathrm{CDF} 4$, water depths in the lake and navigation channel were relatively deep in the remainder of computational grid domain. The land-water interfaces of the grid had gently sloping dry or partially wet areas. Consequently, in the test runs the interior and exterior land boundaries of the $\mathrm{CDF}_{4}$ and adjacent land areas were treated as absorbing boundaries with a low reflection. For wave periods up to $10 \mathrm{sec}$, damping coefficient values from 0 to 1.0 , and widths ranging from 16.4 to $82 \mathrm{ft}$ ( 5 to $25 \mathrm{~m}$ ) were assigned to land and structure boundaries were used in the testing for the existing $\mathrm{CDF}_{4}$ and alternatives investigated. Because the wave damping varies with the water level, different damping layers were used for the three water levels ( $\mathrm{oft}, 7.5 \mathrm{ft}$, and $9.8 \mathrm{ft}(\mathrm{o} \mathrm{m}, 2.3 \mathrm{~m}$ and $3 \mathrm{~m}$ ). The dark brown lines in Figure 4-16 show an example of the damping layers assigned to the open boundary behind the wavemaker, shorelines, structures, and lateral boundaries in a test run with an internal wavemaker. Test runs did not show the existence of long-period IG waves.

\subsection{Modeling details}

\subsubsection{Model calibration parameters}

Field data were unavailable to calibrate and validate the B2D model. Consequently, the key computational parameters used in the $\mathrm{CDF}_{4}$ simulations were based on values used in previous studies. A detailed 
parametric sensitivity study was performed to determine the appropriateness of the recommended parameters. Values of 24 and 0.2 for the friction (Chezy) and Smagorinsky coefficients, respectively, produced the most stable and reasonable solutions over a wide range of test conditions considered, including the three storms and others. The value of the time-step used in the simulations varied with the incident wave and water level inputs, ranging from $0.05 \mathrm{sec}$ to $0.1 \mathrm{sec}$. The effects of Chezy and Smagorinsky coefficients and damping layers on model results and computational stability based on a sensitivity study were described in Sections 4.3 and 4.4. In the sensitivity runs (e.g., blind tests), B2D was intentionally set up using non-default parameters to determine the values of calibration parameters most appropriate for this study. This investigation confirmed and suggested that in projects where no field data are available, the model could be used with default parameters. Tests indicated the model was insensitive to computational parameter settings and that predominant factors in modeling were the incident forcing conditions and water levels. Additional simulations were made using different values of non-storm conditions to ascertain the effect of numerical parameters on model results. Overall, the final calibration parameters agreed with the recommended default values for B2D modeling used in similar wavestructure interaction studies (Demirbilek et al. 2016, 2015a,b, 2007; Nwogu and Demirbilek 2001).

\subsubsection{Output locations and files}

B2D results were saved over the entire grid and at the special locations of interest called "wave probes" or gauges. Output includes temporal and spatial solution files over the entire grid. Each condition was simulated on the B2D grid described earlier (Figure 4-8). The temporal and spatial output files of the mean water level (i.e., wave setup), mean wave direction, significant wave height, mean velocity (current), and time series of water surface elevation and pressure at the probe locations were post-processed and analyzed. The B2D model interface in the Surface-water Modeling System (SMS) (http://www.aquaveo.com) was used to view, extract, and postprocess model results (i.e., wave parameters such as wave height, period, direction, water level, and wave-induced current). Additional analyses of the model time-series solution files saved at specified probes were performed using Matlab and Fortran codes. These codes and SMS post-processing capabilities were used to develop estimates of wave parameter statistics, wave runup and overtopping in the time and frequency domains. 


\subsubsection{Results for storm conditions}

Three storm conditions in Table 4-1 were simulated with B2D. The meteorological (or from) direction convention was used to define the direction of waves and winds for the three storms simulated. In this convention, the direction is measured clockwise with respect to true north, where north is o deg. It was noted in the model calibration/validation section that incident wave directions of the three storms at the $\mathrm{B} 2 \mathrm{D}$ grid open boundary were essentially the same ( $247 \pm 1 \mathrm{deg})$. The sensitivity study showed that waves could reach the project area from various directions, but the Perimeter Dike and CDF4 interior were affected largely by incident waves in Lake Erie from the southwest direction (247 deg range).

Figures 4-17, 4-18, and 4-19 show calculated wave height fields for three storms and the existing condition of the $\mathrm{CDF} 4$. The spatial variation in wave fields looks similar because the directions and periods of storm waves in the lake were alike. As shown in Table 4-1, only the wave heights and water levels of each storm were different. Consequently, model results from the three storms show minor changes in the spatial wave height fields. One common feature of waves propagating toward the $\mathrm{CDF}_{4}$ is wave focusing on the northern half of the Perimeter Dike. Model results for the three storms indicate wave focusing on the north half (Legs 3, 4, and 5) of the curved dike. A weak intensification of waves (focusing) occurred on the south-half of the Dike (Legs 1 and 2) as well. The middle and north sections of the curved dike (with more severe wave focusing) experienced the most frequent and greatest wave overtopping.

The intensification in wave heights is a consequence of wave-dike interaction, indicating stronger wave refraction, wave reflection, and diffraction by the dike as represented by B2D model. In B2D, water levels associated with each storm also play a role on waves near the dike. Because the peak water levels used for three storms were similar, the effects of water levels on waves at the dike were similar. 
Figure 4-17. Wave height field of Storm Condition 1 over the model grid.

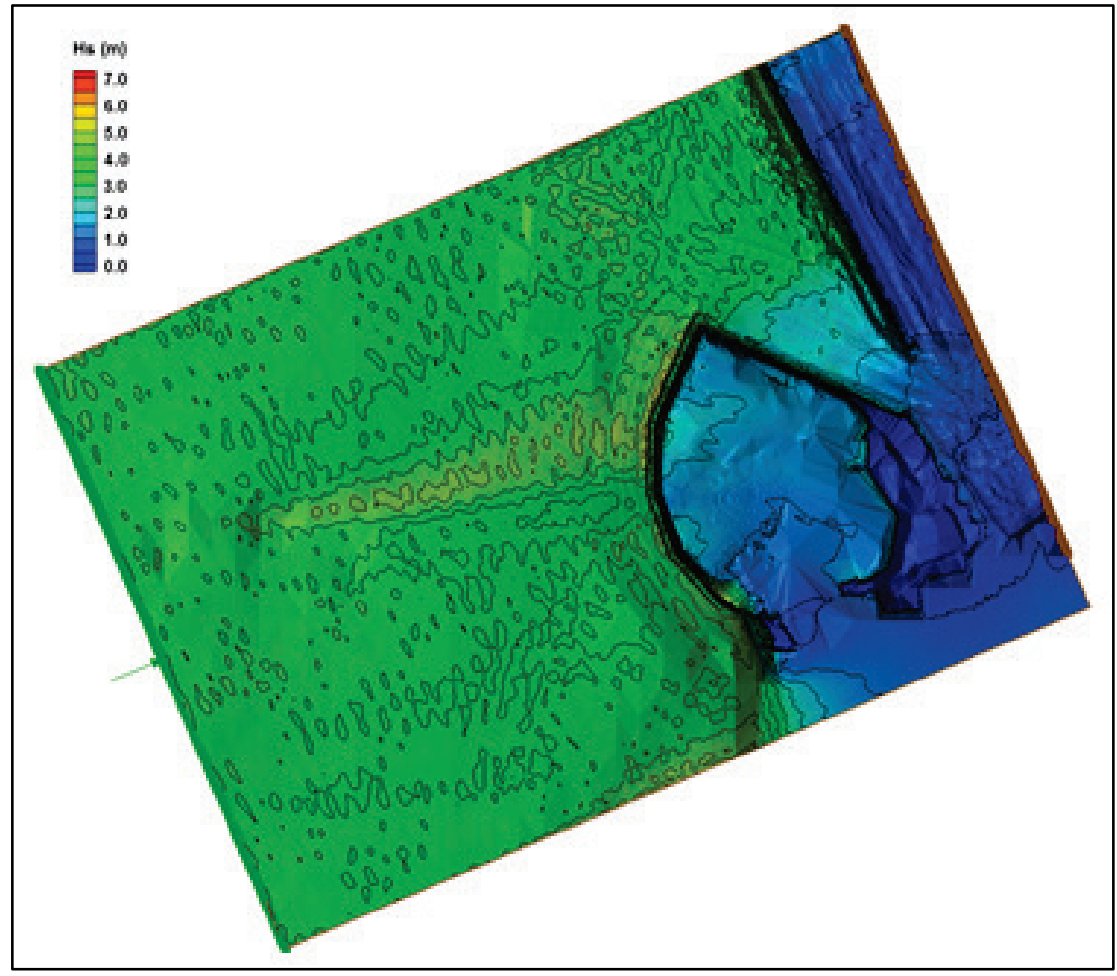

Figure 4-18. Wave height field of Storm Condition 2 over the model grid.

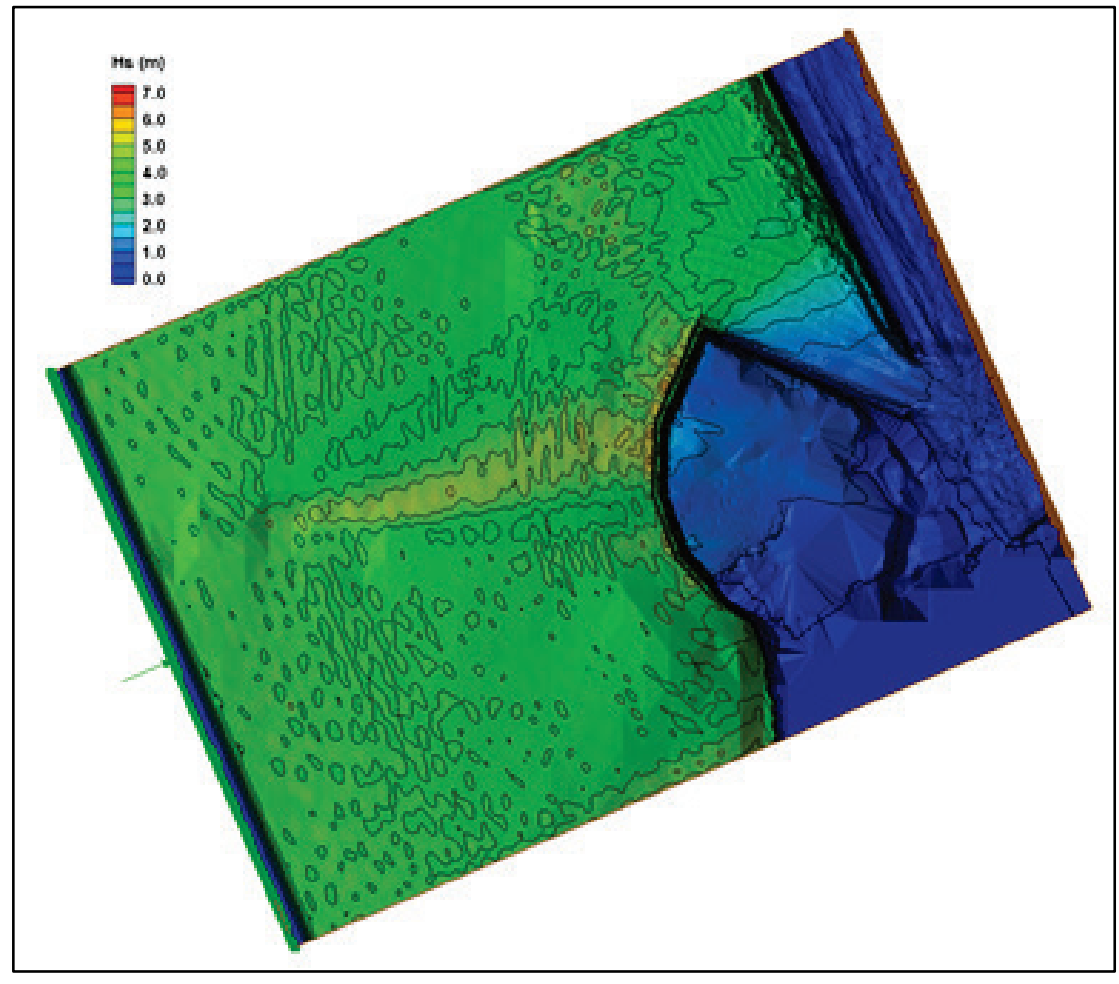


Figure 4-19. Wave height field of Storm Condition 3 (1982 storm) over the model grid.

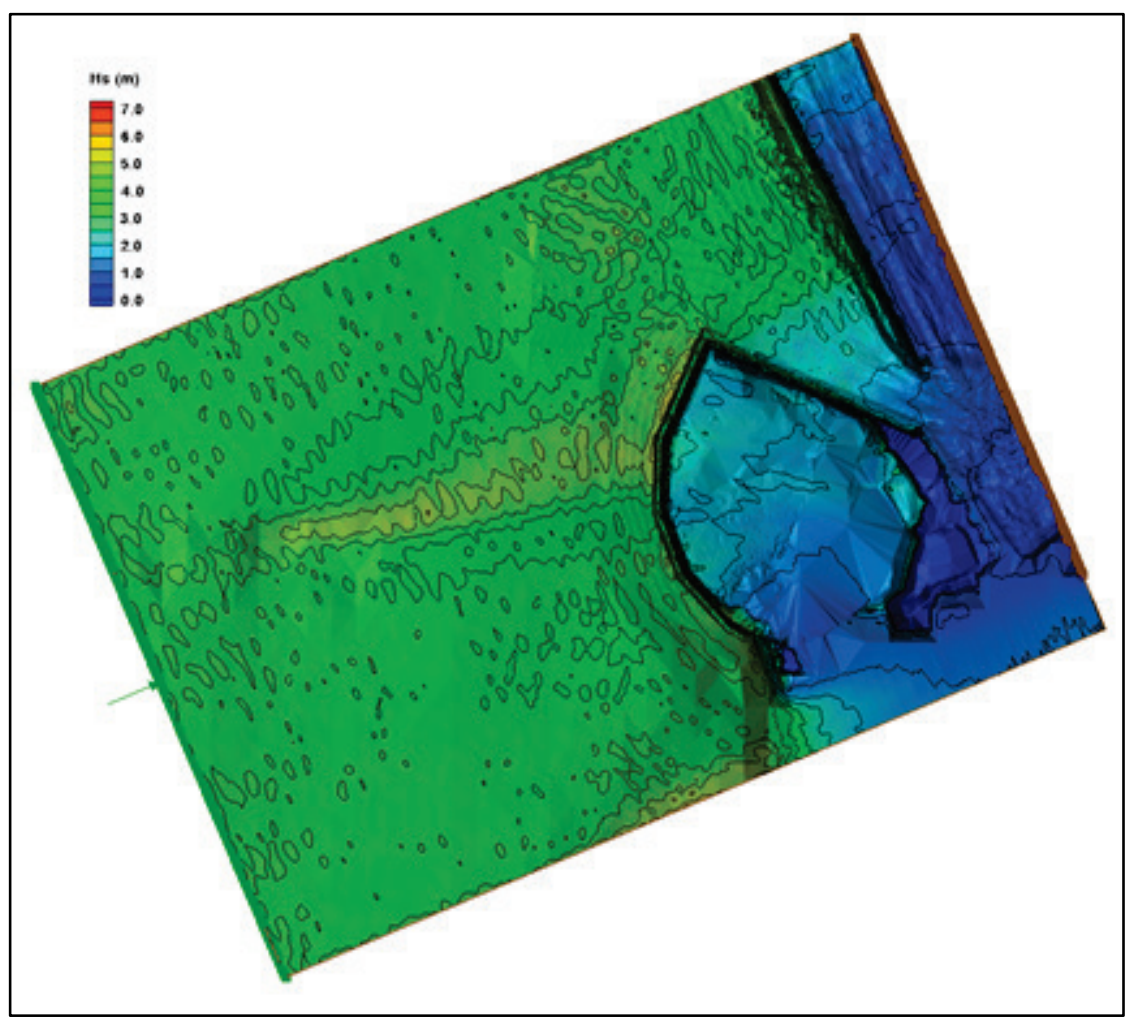

The strong wave focusing seen in Figures 4-17, 4-18, and 4-19 was not apparent in the CMS-Wave simulations. This is because wave-action models such as CMS-Wave generally have a comparatively weaker wave reflection and diffraction than phase-resolving models (Lin et al. 2008). In addition, different grid resolution was used for the two wave models. CMS-Wave results indicated large wave heights near the mid-section of the dike (Legs 2 to 4), while B2D showed large waves could occur along Legs 2 to 5 .

Figures 4-20, 4-21, and 4-22 show more details of waves in and around the CDF4. These snapshots of sea surface elevation animation represent the condition of the sea state at different instants in time for the entire model grid. These instantaneous temporal images show details of spatial variation in waves outside and inside the $\mathrm{CDF}_{4}$. The vertical scale was magnified in the animations to show detailed features in spatial variability, location, and intensity of wave surface outside and in the interior of CDF4. Detailed estimates of wave overtopping and calculated rates of overtopping for three simulated storms are discussed in the next section in this chapter. 
Figure 4-20. A snapshot of Storm Condition 1 sea surface elevation around the CDF4.

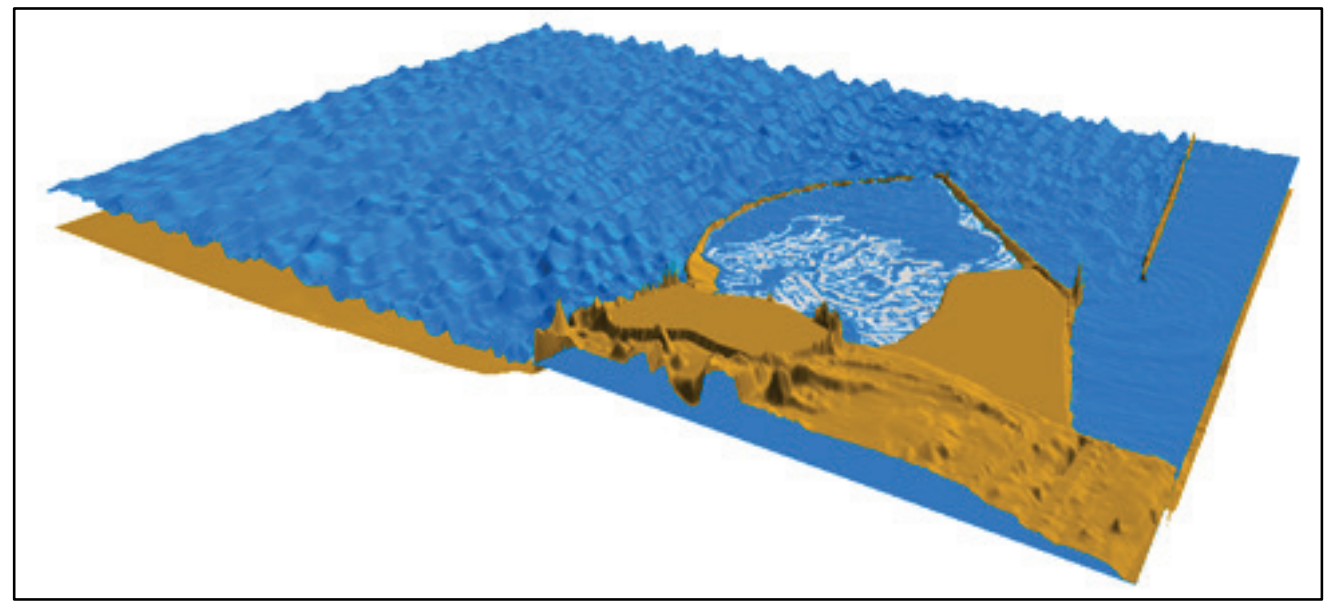

Figure 4-21. A snapshot of Storm Condition 2 sea surface elevation around the CDF4.

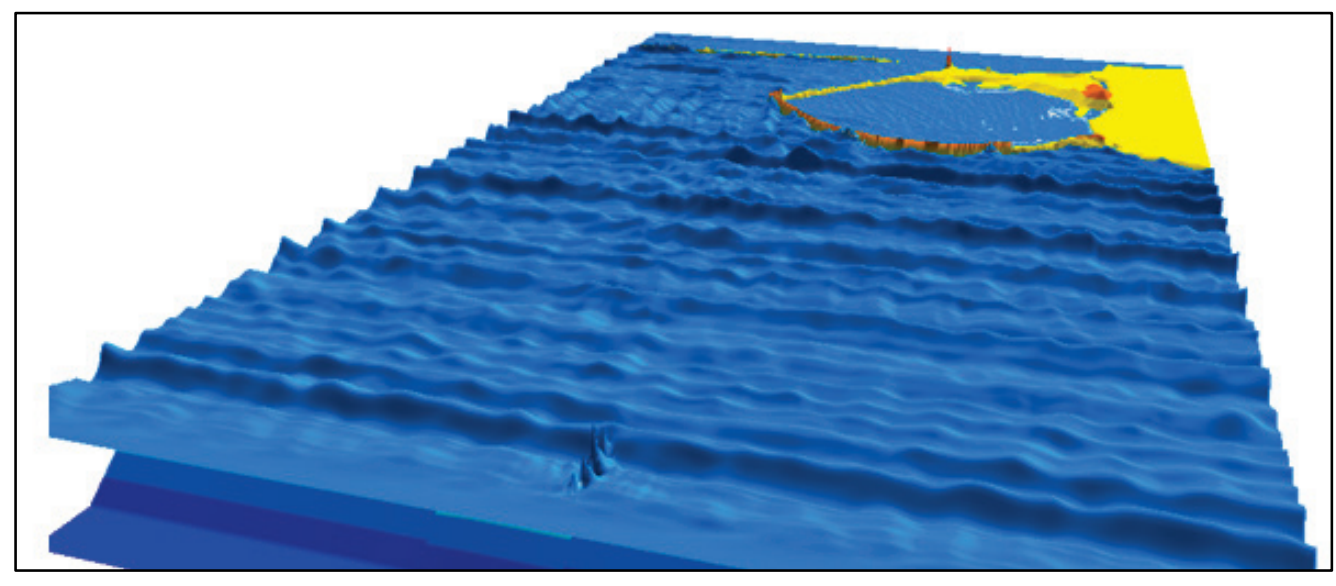

Figure 4-22. A snapshot of Storm Condition 3 sea surface elevation in and around CDF4.

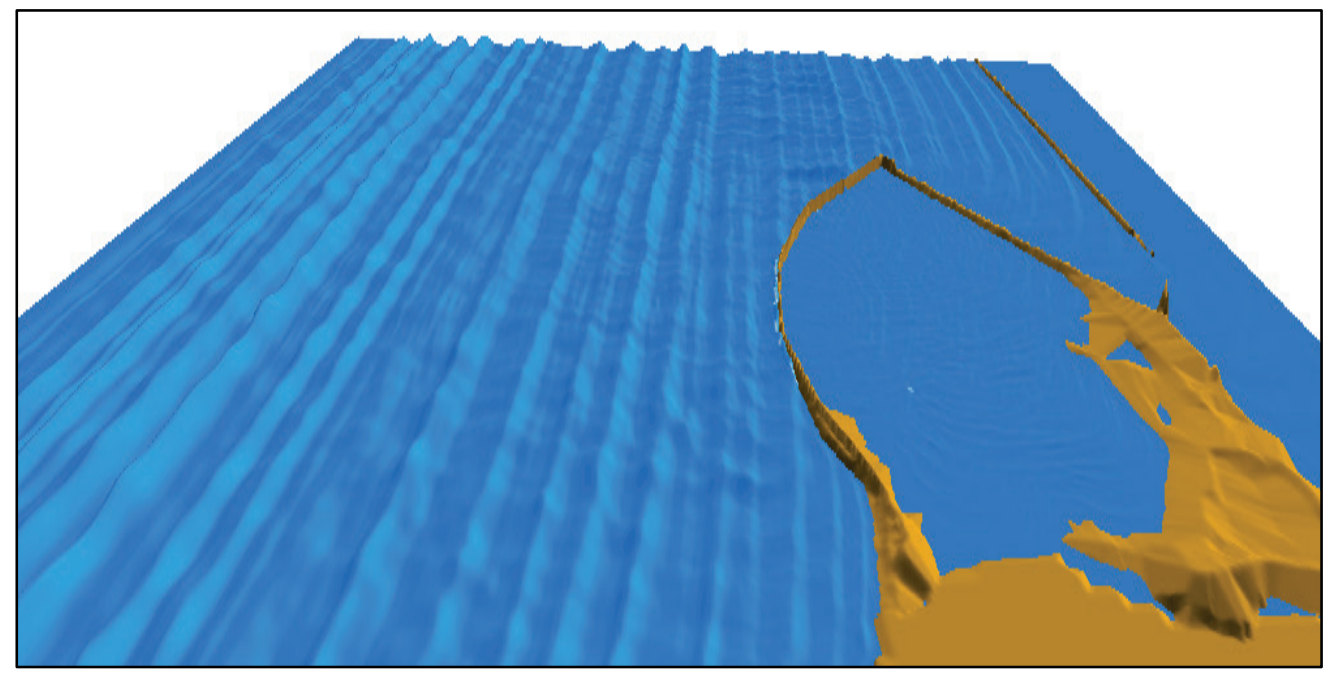




\subsubsection{Waves inside the CDF4}

Figures 4-23, 4-24, and 4-25 are the snapshots of waves in the interior of $\mathrm{CDF} 4$. These figures highlight differences between the calculated wave fields outside and inside CDF4. Figure 4-25 is a zoomed image of the overtopped waves behind the structure (in the interior of $\mathrm{CDF}_{4}$ ), and it shows reformation and propagation of waves inside the $\mathrm{CDF}_{4}$ system. The height of these waves is reduced substantially compared to larger waves out in the lake and the height of focusing waves along the front face of the curved dike. Strong and violent interaction between waves and dike structure results in wave transmission, runup/overtopping, breaking, and dissipation through and over the structure. These processes cause waves to lose energy substantially. Waves that are able to get inside the $\mathrm{CDF} 4$ cannot grow in the shallow interior area of $\mathrm{CDF} 4$ with a limited fetch, and wind wave growth is not considered in B2D, and majority of waves break as shown by foam in the images.

Figure 4-23. Progression of waves in the interior of CDF4.

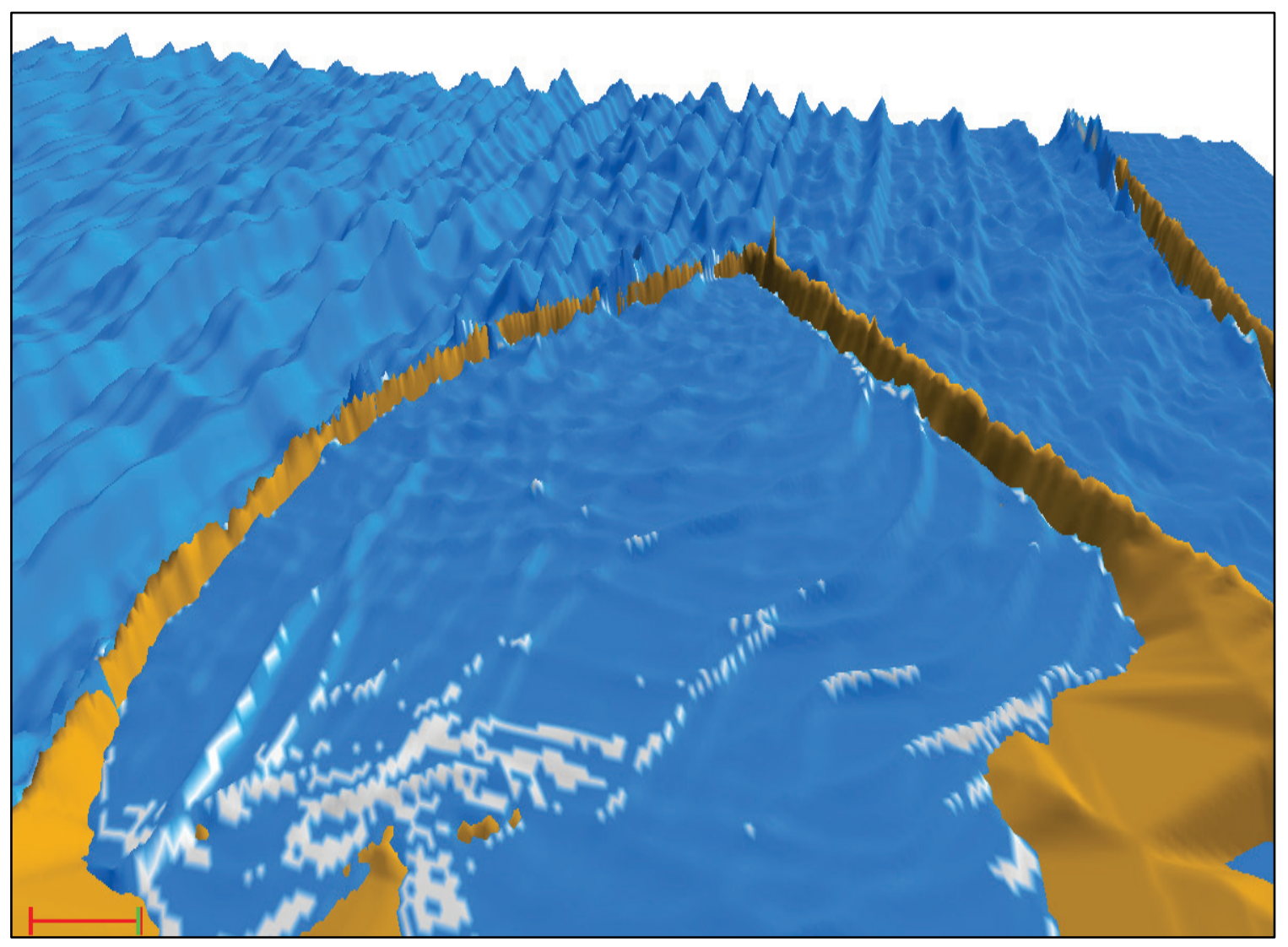


Figure 4-24. A zoomed image of interior waves in south side of CDF4.

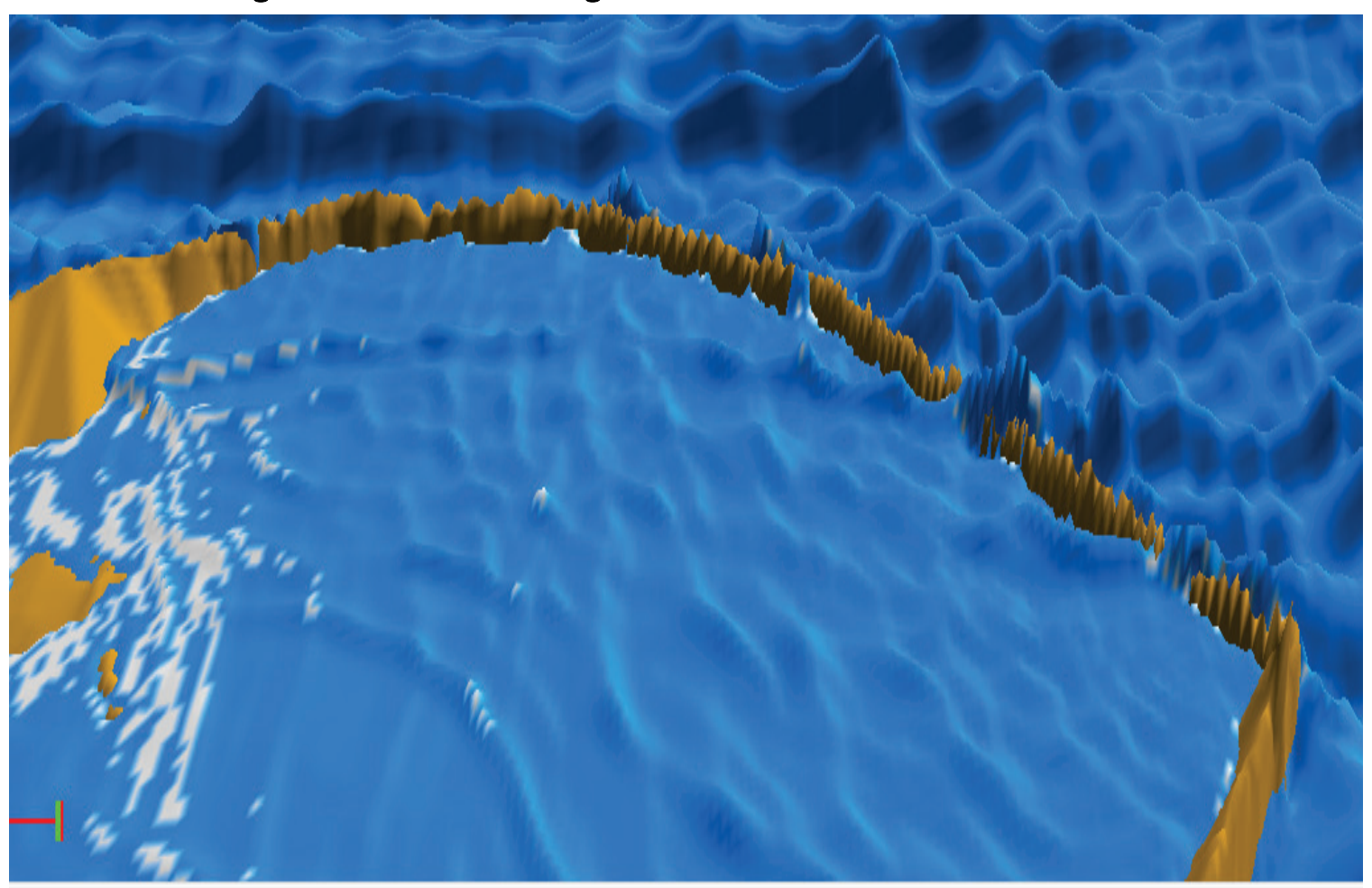

Figure 4-25. A closeup of interior waves on north side near the V-section of CDF4.

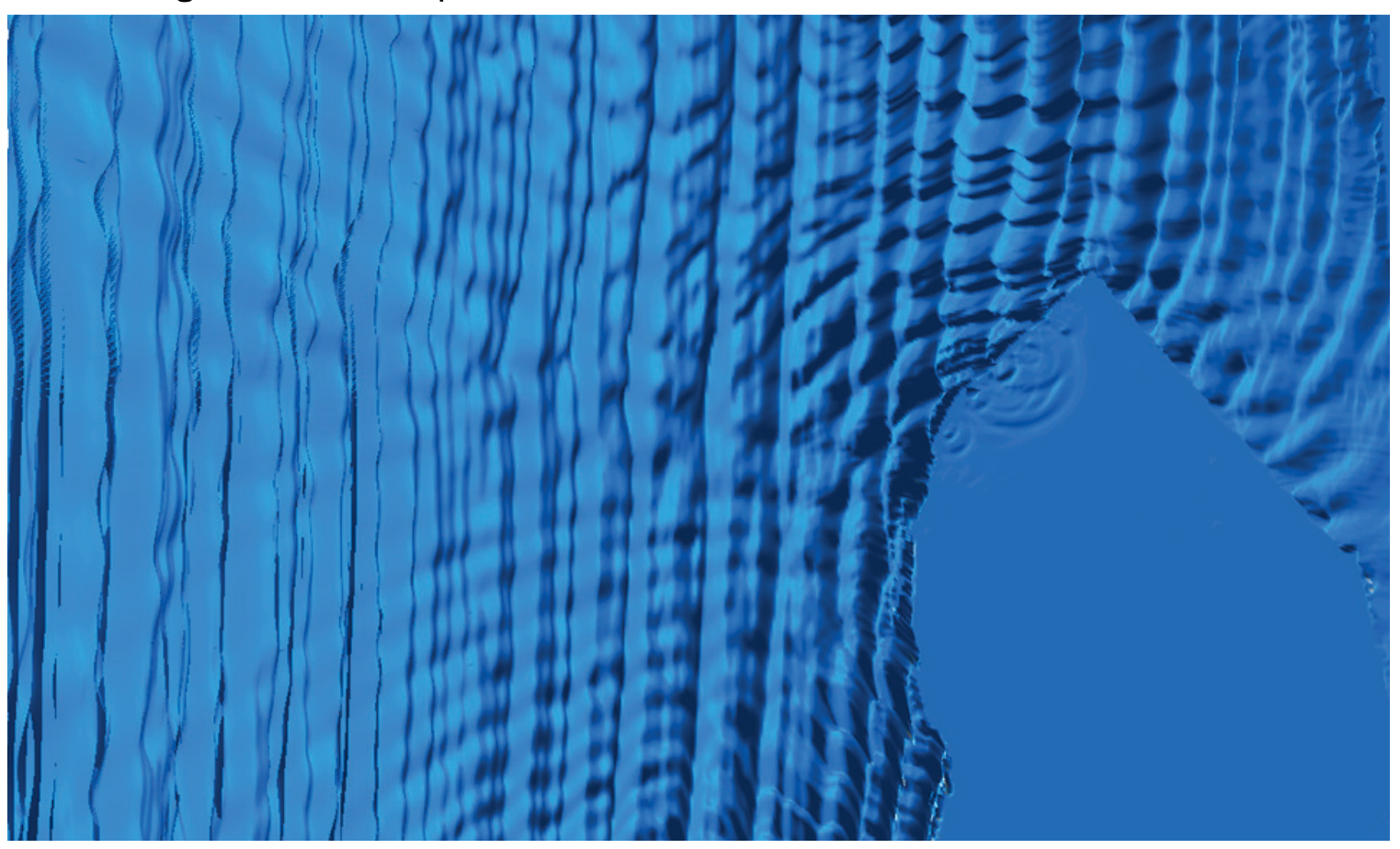




\subsubsection{Images of instantaneous wave overtopping}

As noted in Section 4.3, there are gaps and low spots throughout the existing dike structure. Wave overtopping occurred at and near these locations, and overtopping positions changed with the water level and wave height along the front face of the dike. In general, the dike segments (legs) experiencing increased wave focusing had the most severe overtopping. Simulations indicated wave overtopping increasing at the higher water levels with increasing wave height.

Figures 4-26 through 4-49 are snapshots of wave fields displaying spatial details of waves in the lake side along the front face of the curved dike. These figures show wave overtopping captured during a $4000 \mathrm{sec}$ long Storm Condition 2 simulation along the curved dike. Model results indicate the location of overtopping along the Perimeter Dike is variable. There is persistent overtopping of the mid-section of the dike. Segment 1 in the south side experiences the least overtopping followed by Segment 2. In this image of Storm Condition 2, Segments 3, 4, and 5 from the middle to the V-section of the curved dike consistently experienced the severest overtopping. There is no evidence of waves overtopping any section of the SEAB.

Figure 4-26. Overtopping at dike south and middle segments.

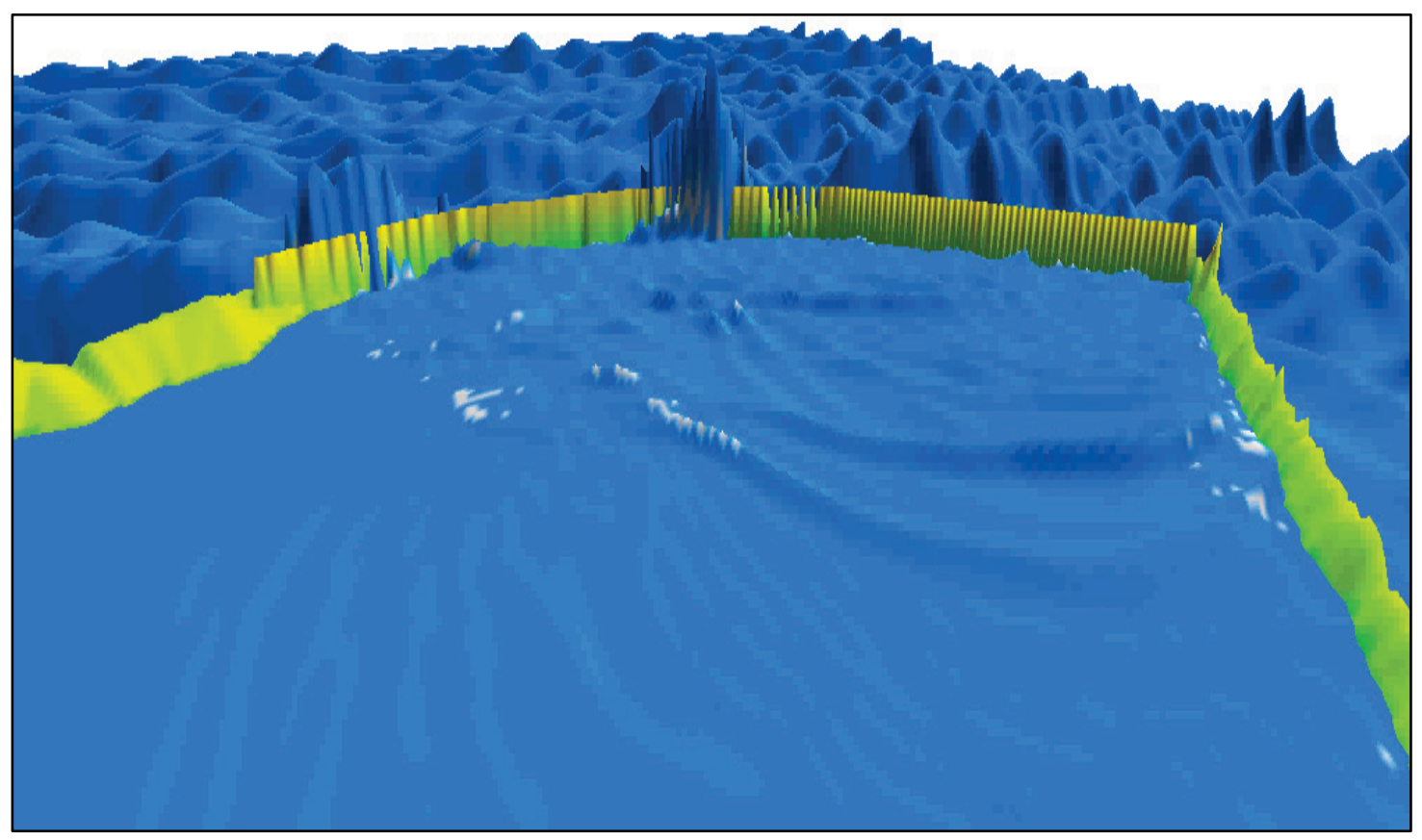


Figure 4-27. Overtopping of dike south segments.

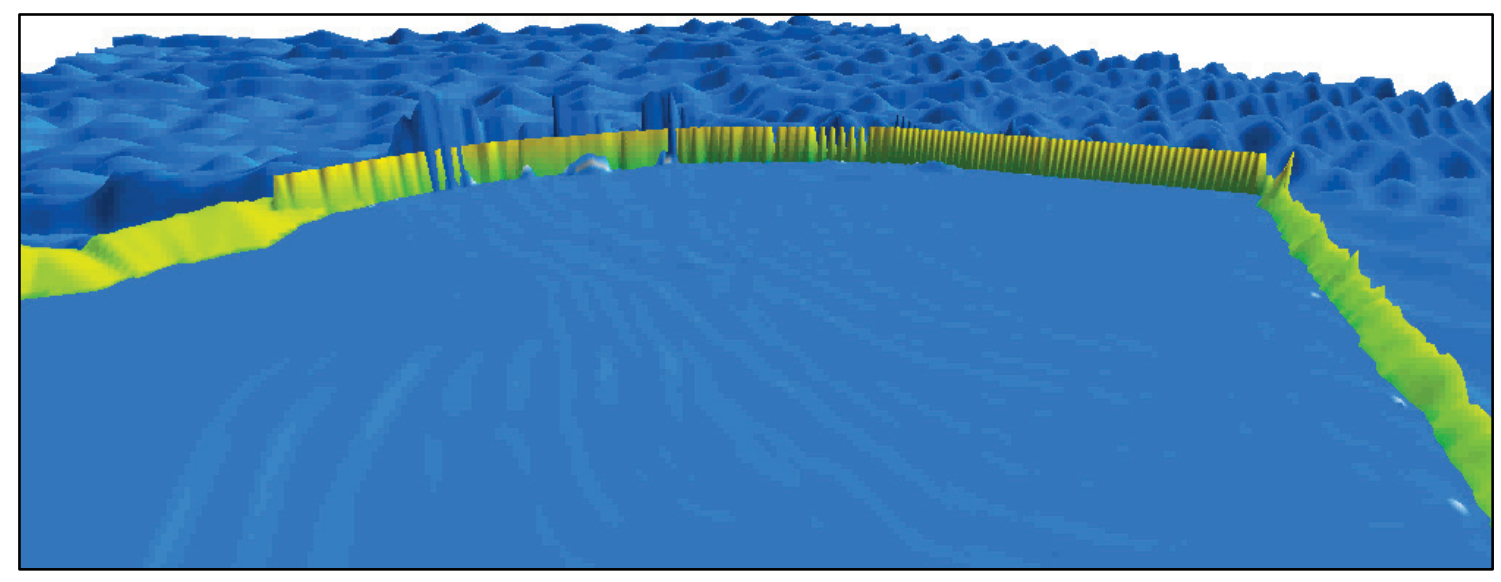

Figure 4-28. Overtopping at dike segment 4 (scene 1).

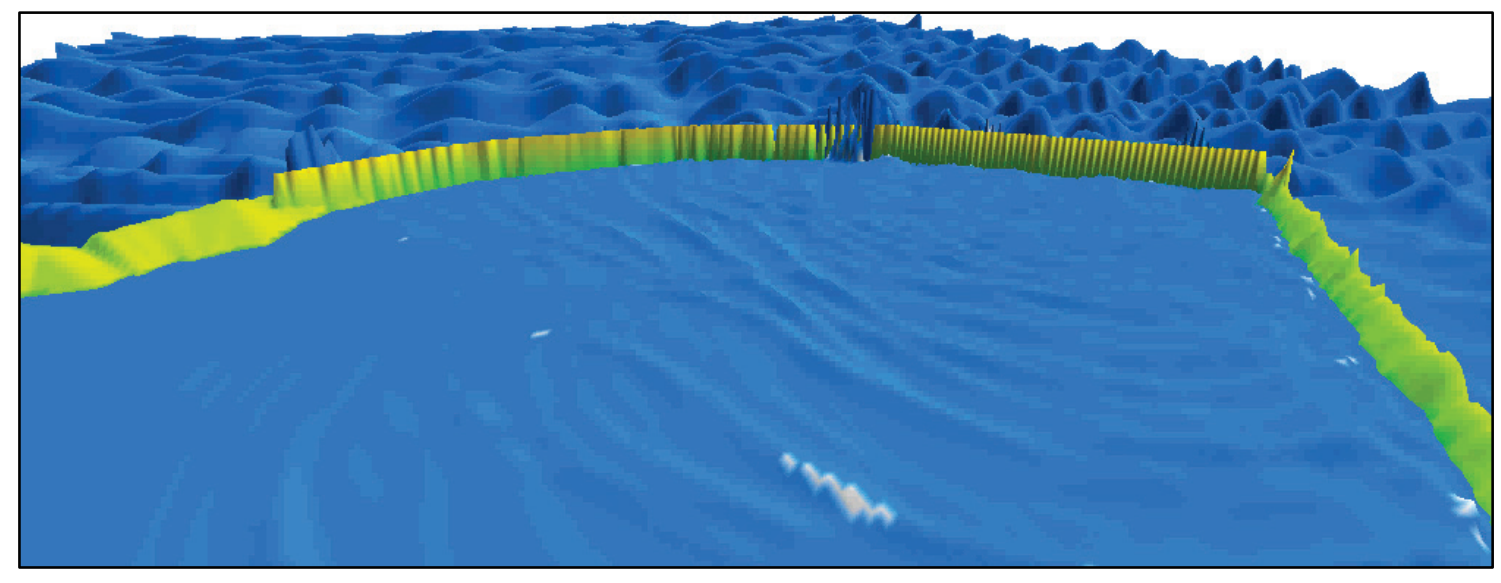

Figure 4-29. Overtopping at dike segments 2, 3, 4, and 5 (scene 1).

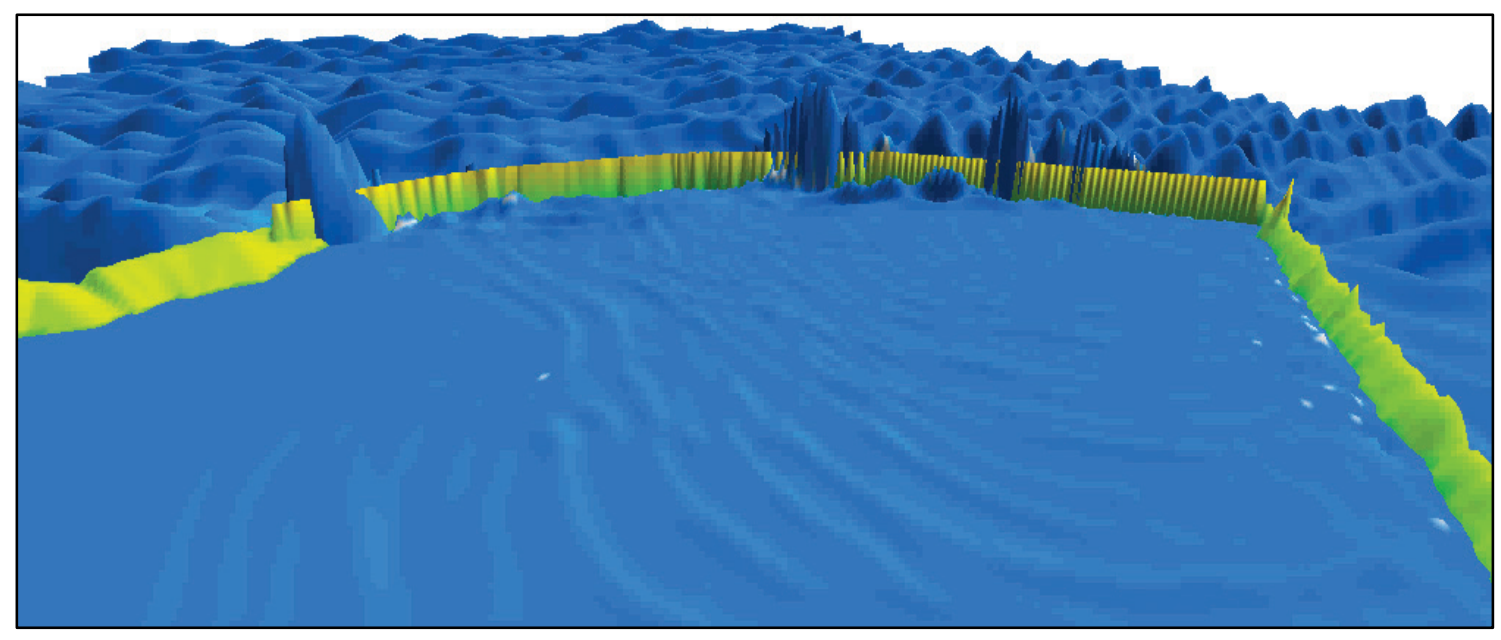


Figure 4-30. Overtopping at dike segment 5 (scene 1).

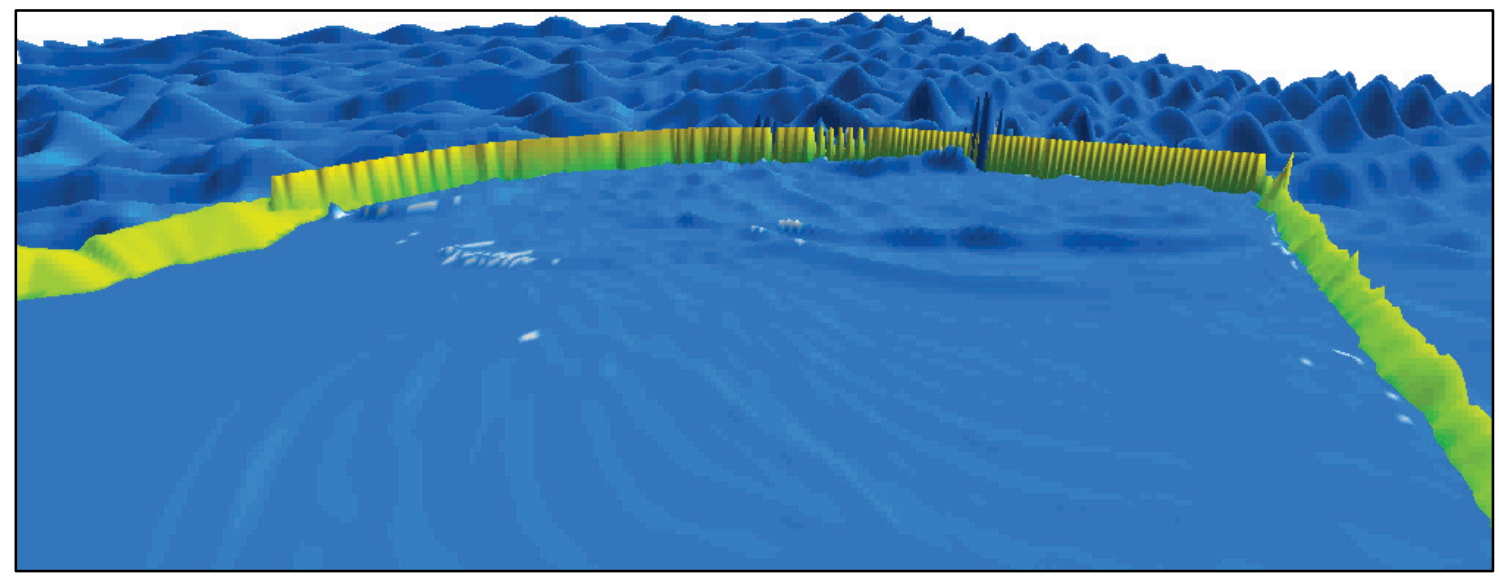

Figure 4-31. Overtopping at dike segments 2 and 3 (scene 1).

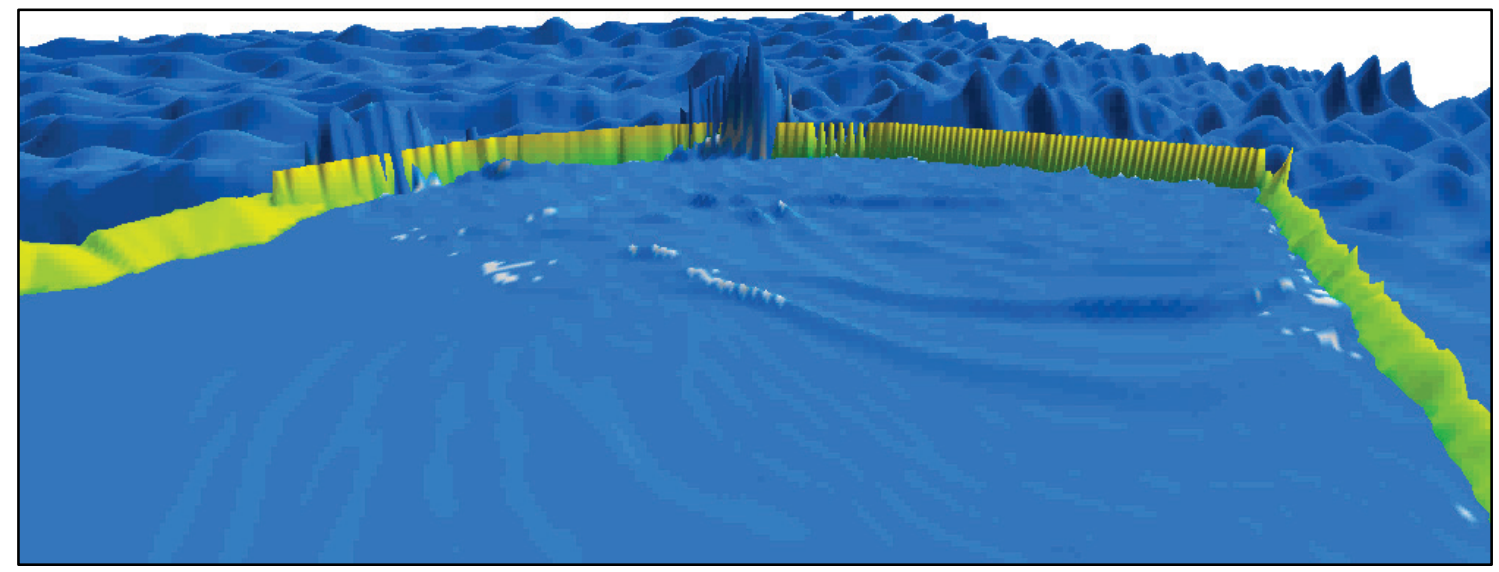

Figure 4-32. Overtopping at dike segment 5 (scene 2).

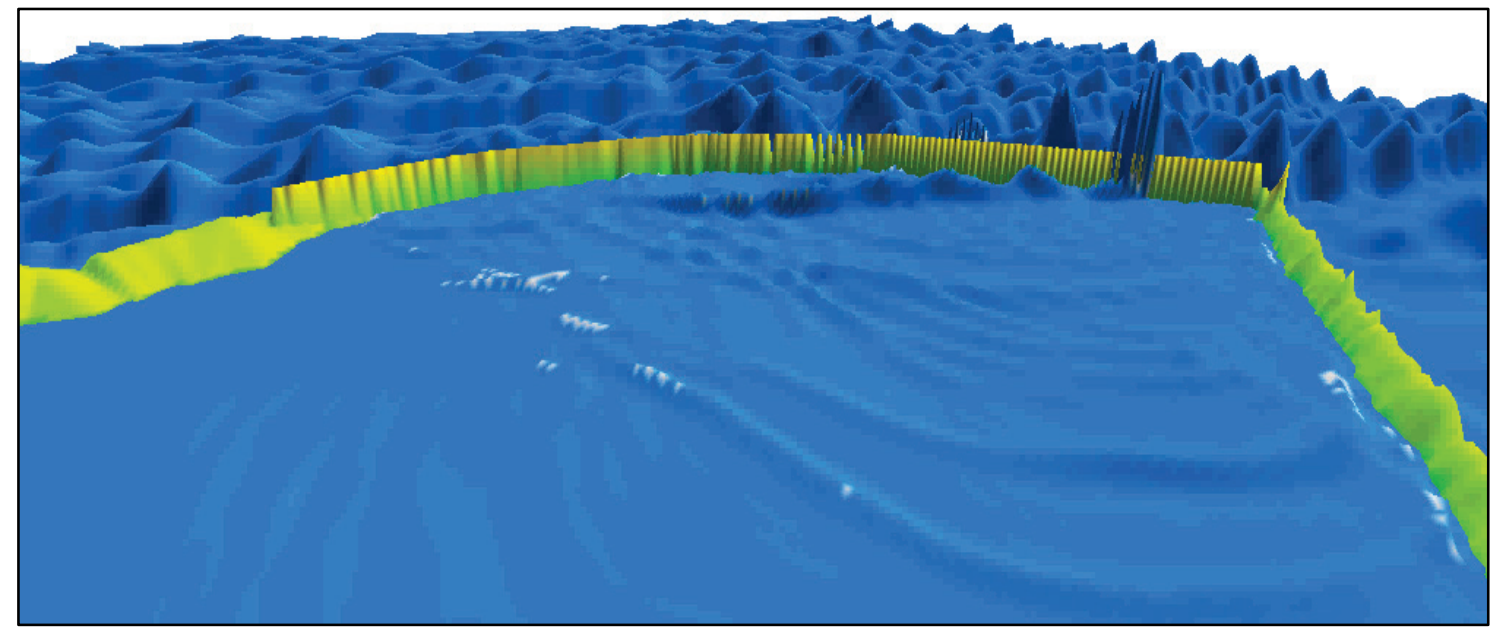


Figure 4-33. Overtopping at dike segments 2, 3, and 4.

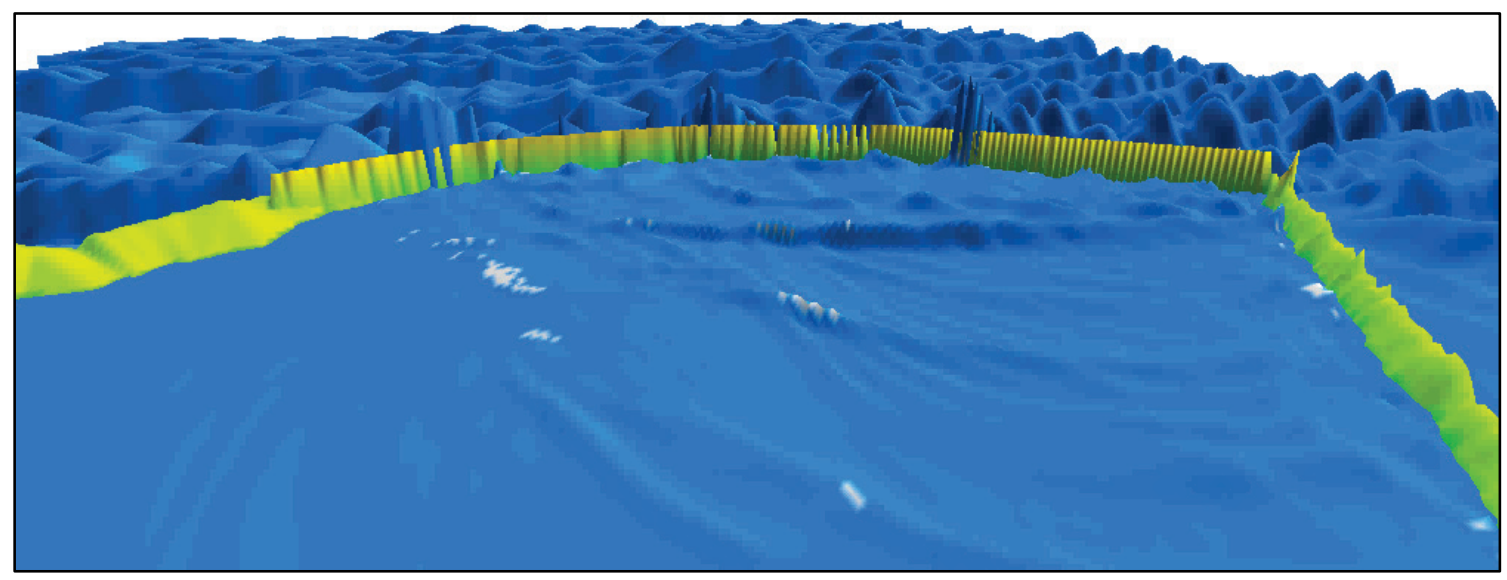

Figure 4-34. Overtopping at dike segments 3 and 4.

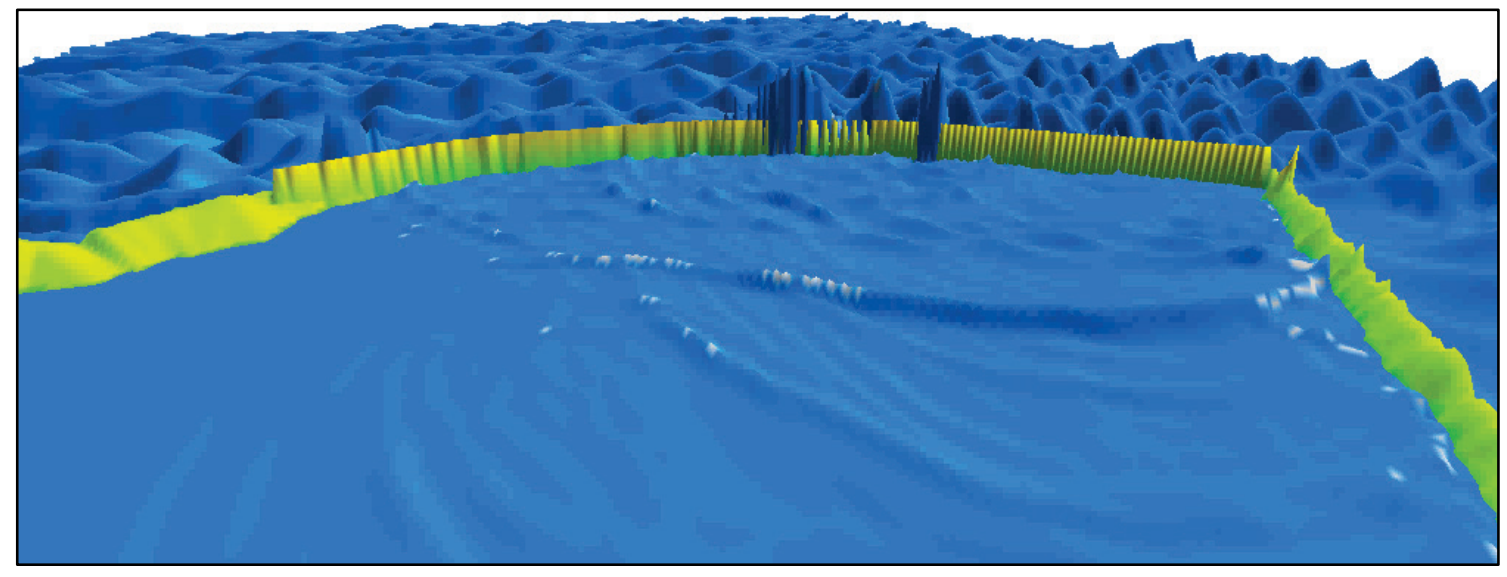

Figure 4-35. Overtopping at dike segment 5 (scene 3).

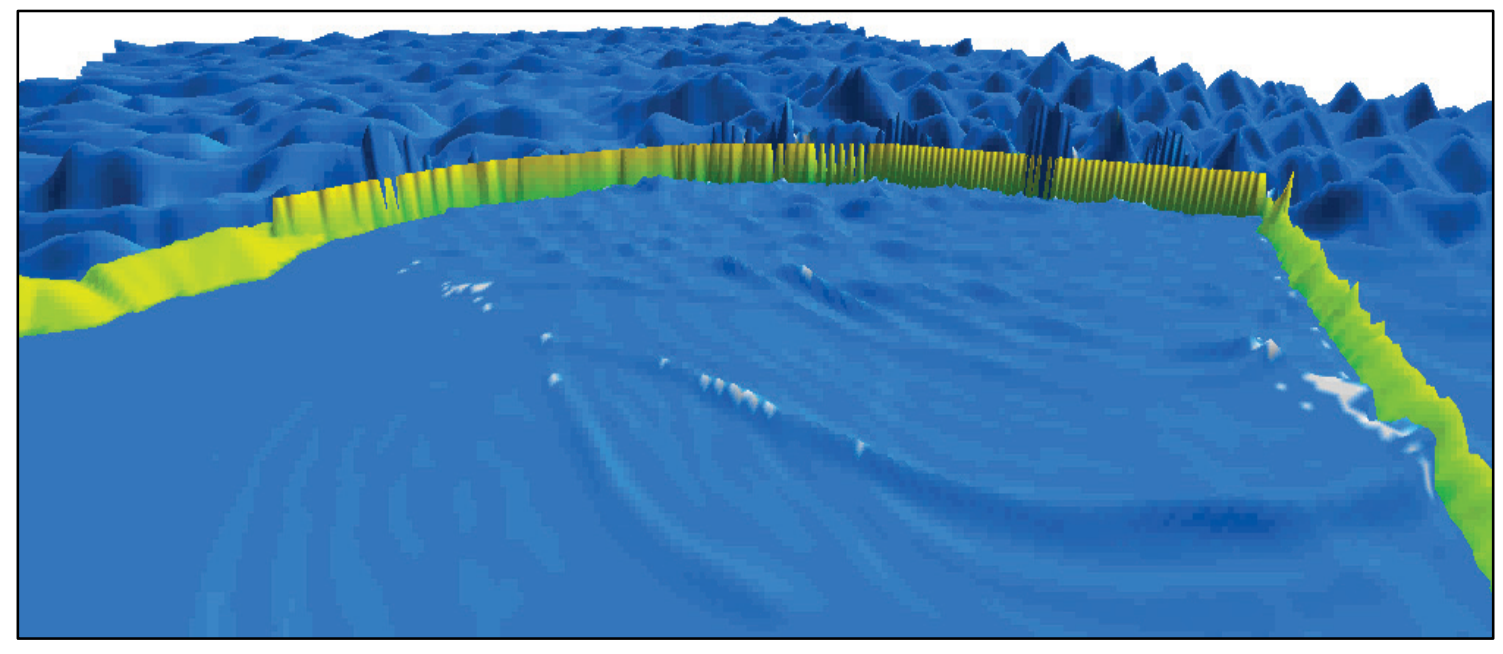


Figure 4-36. Overtopping at dike segments 2 and 3 (scene 2).

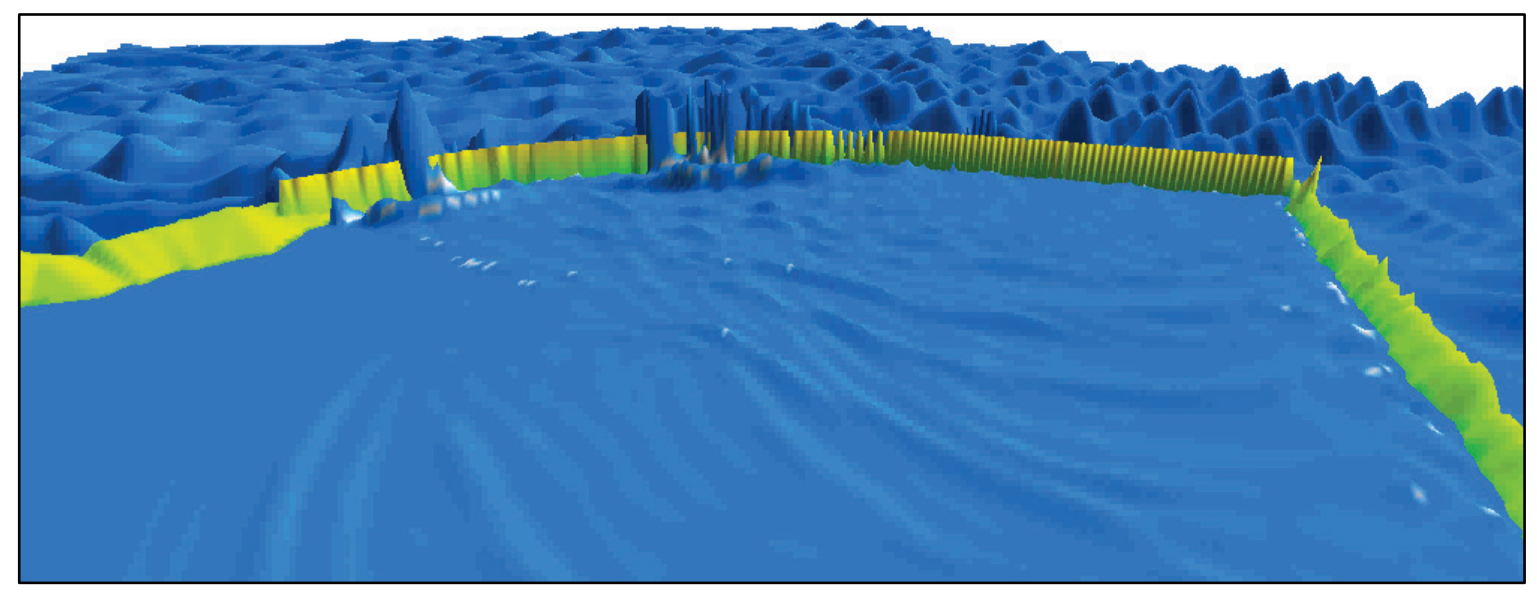

Figure 4-37. Overtopping at dike segments 4 and 5 (scene 1).

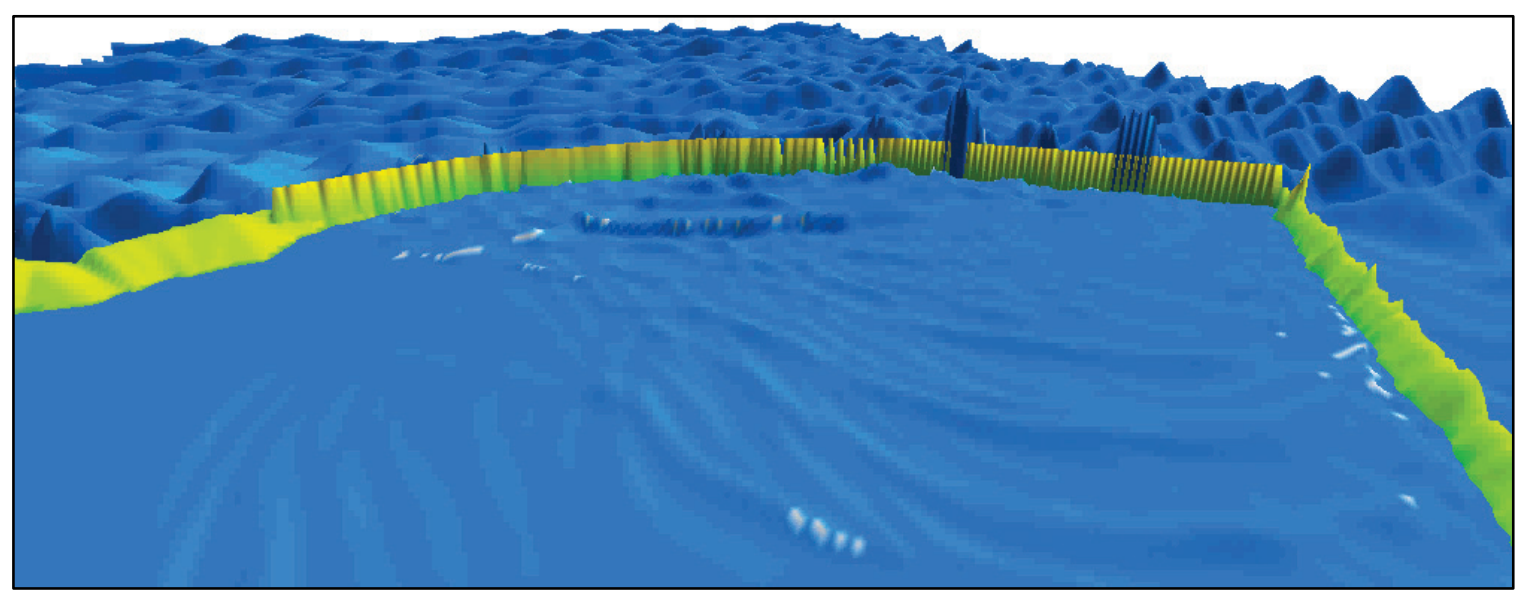

Figure 4-38. Overtopping at dike segments 4 and 5 (scene 2).

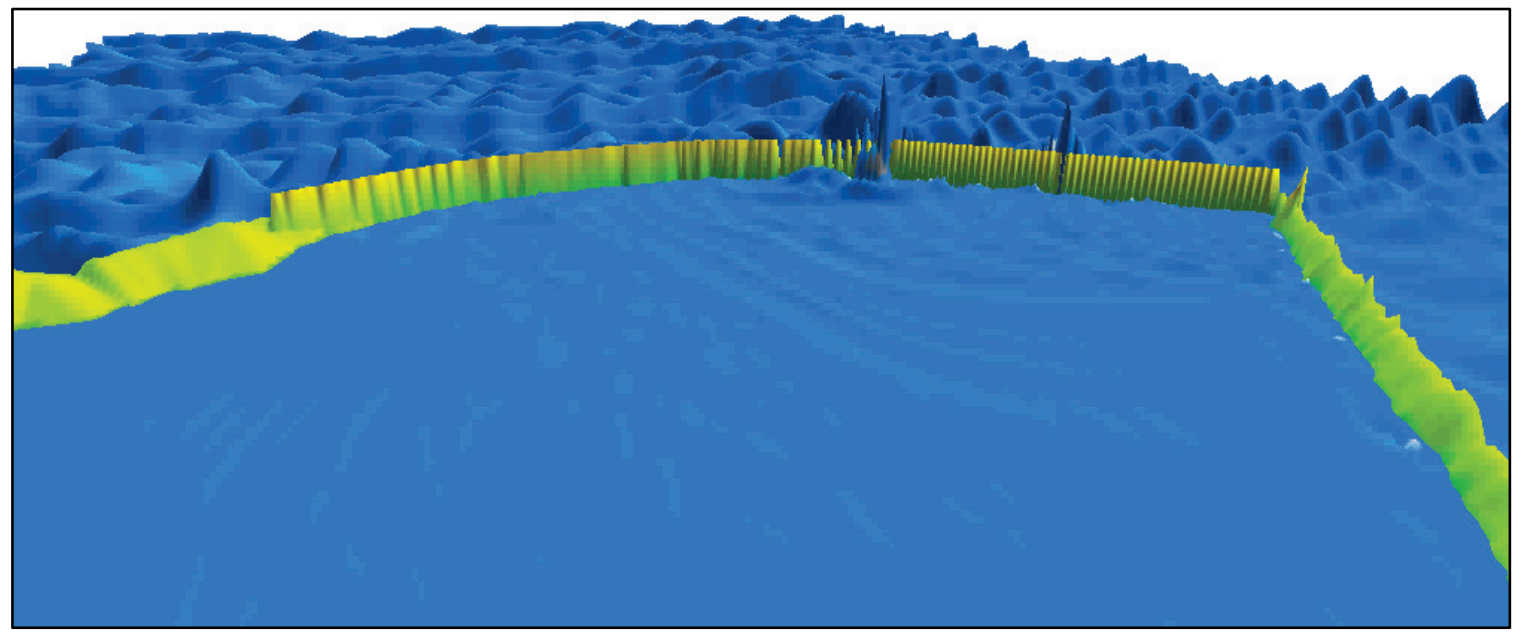


ERDC/CHL TR-17-18

81

Figure 4-39. Overtopping at dike segment 4 (scene 2).

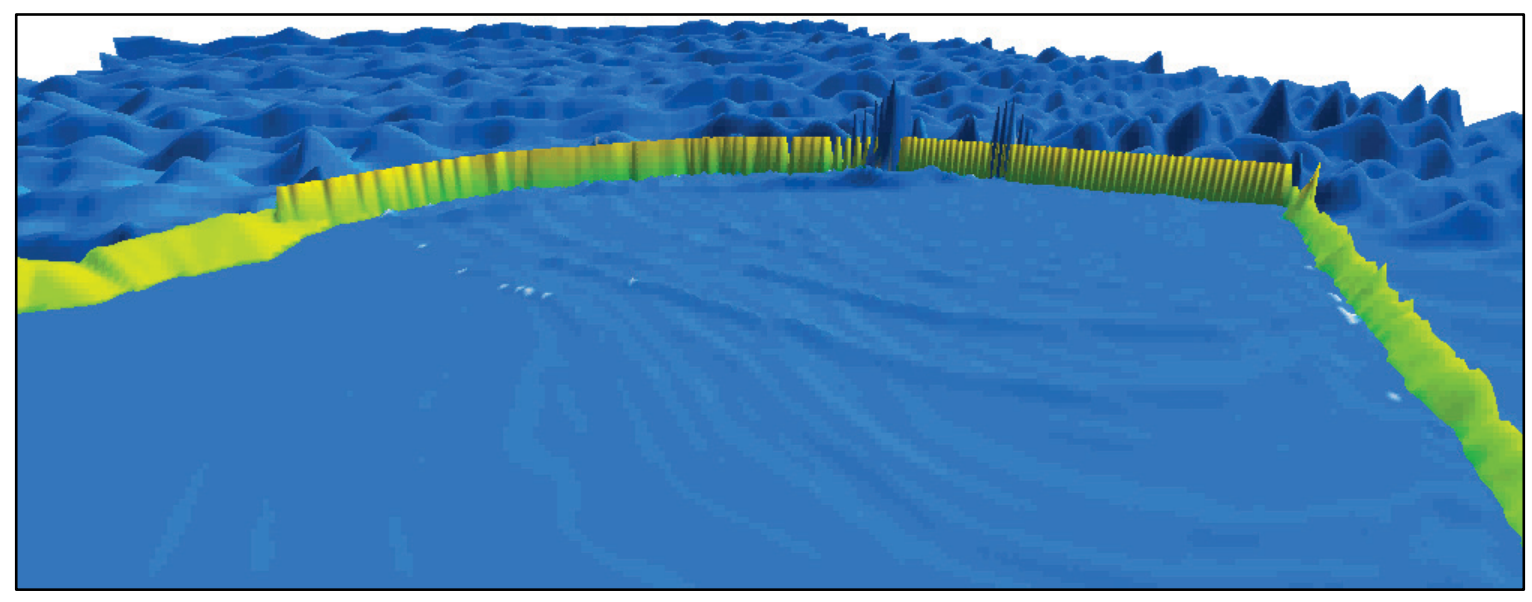

Figure 4-40. Overtopping at dike segments 2 and 5.

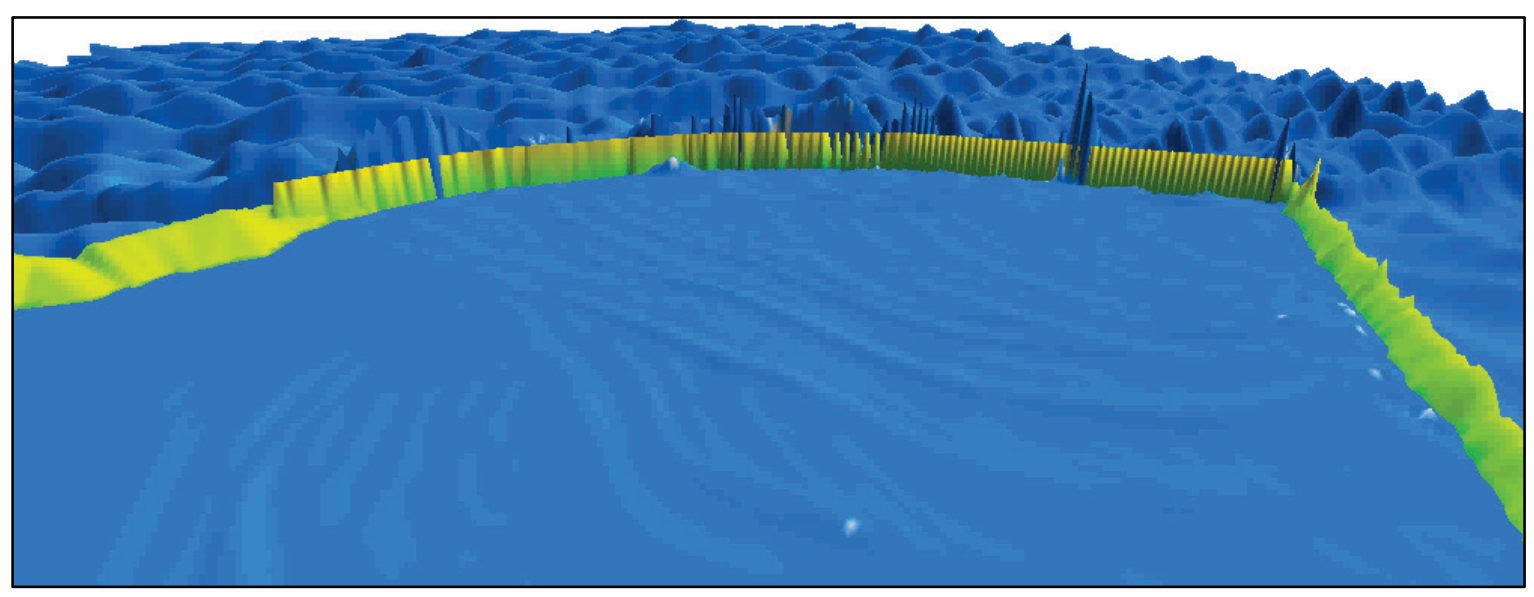

Figure 4-41. Overtopping at dike segment 5 (scene 4). 
ERDC/CHL TR-17-18

82

Figure 4-42. Overtopping at dike segments 2, 3, and 5.

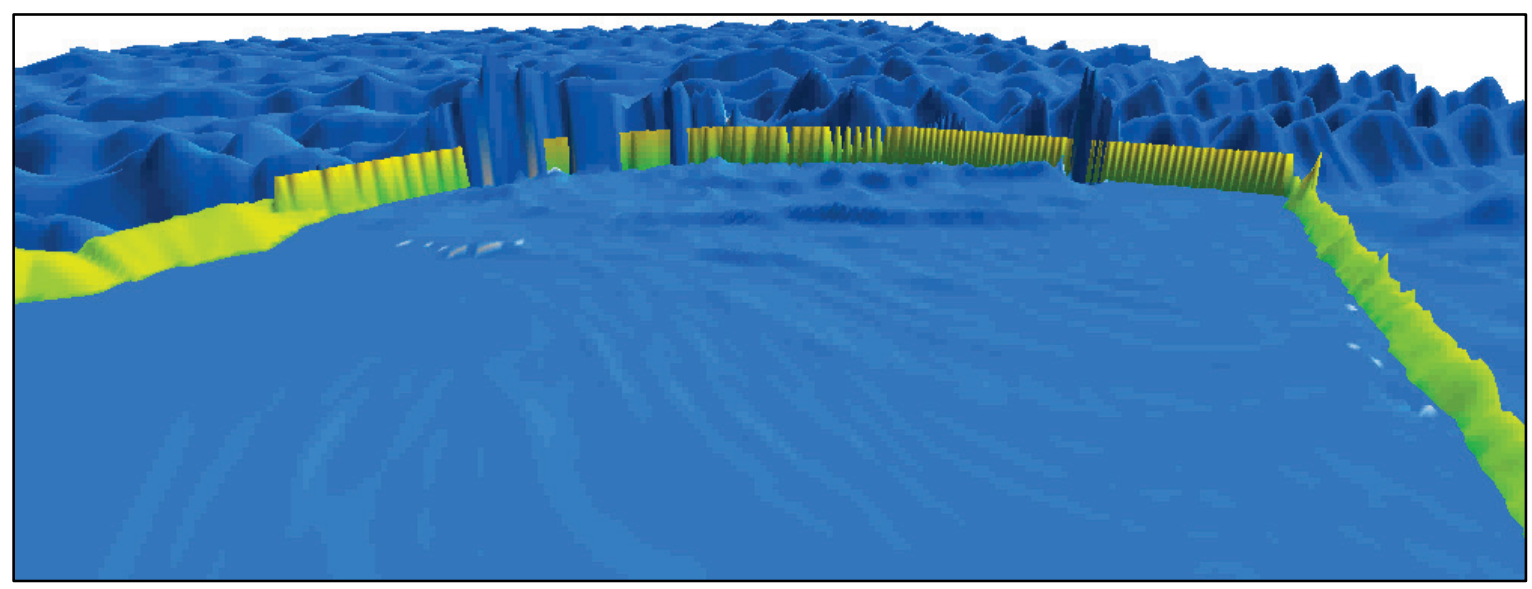

Figure 4-43. Overtopping at dike segments 2,4 , and 5.

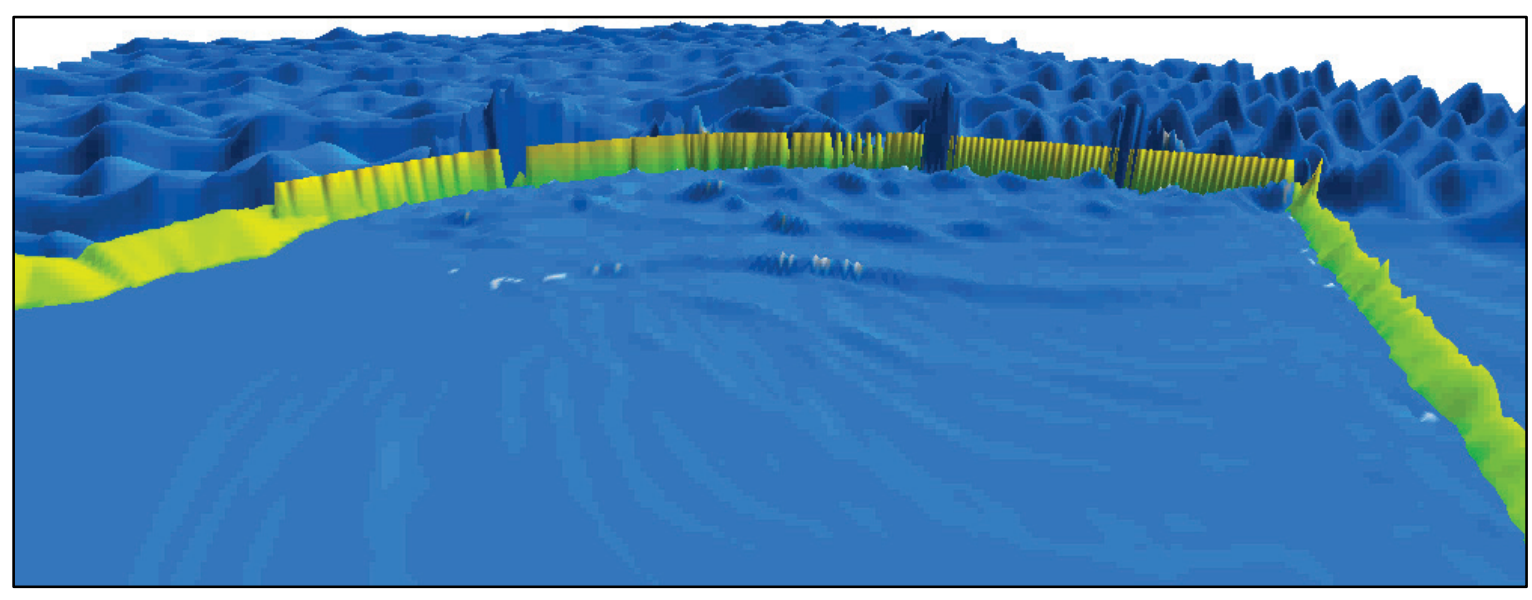

Figure 4-44. Overtopping at dike segments 4 and 5 (scene 3).

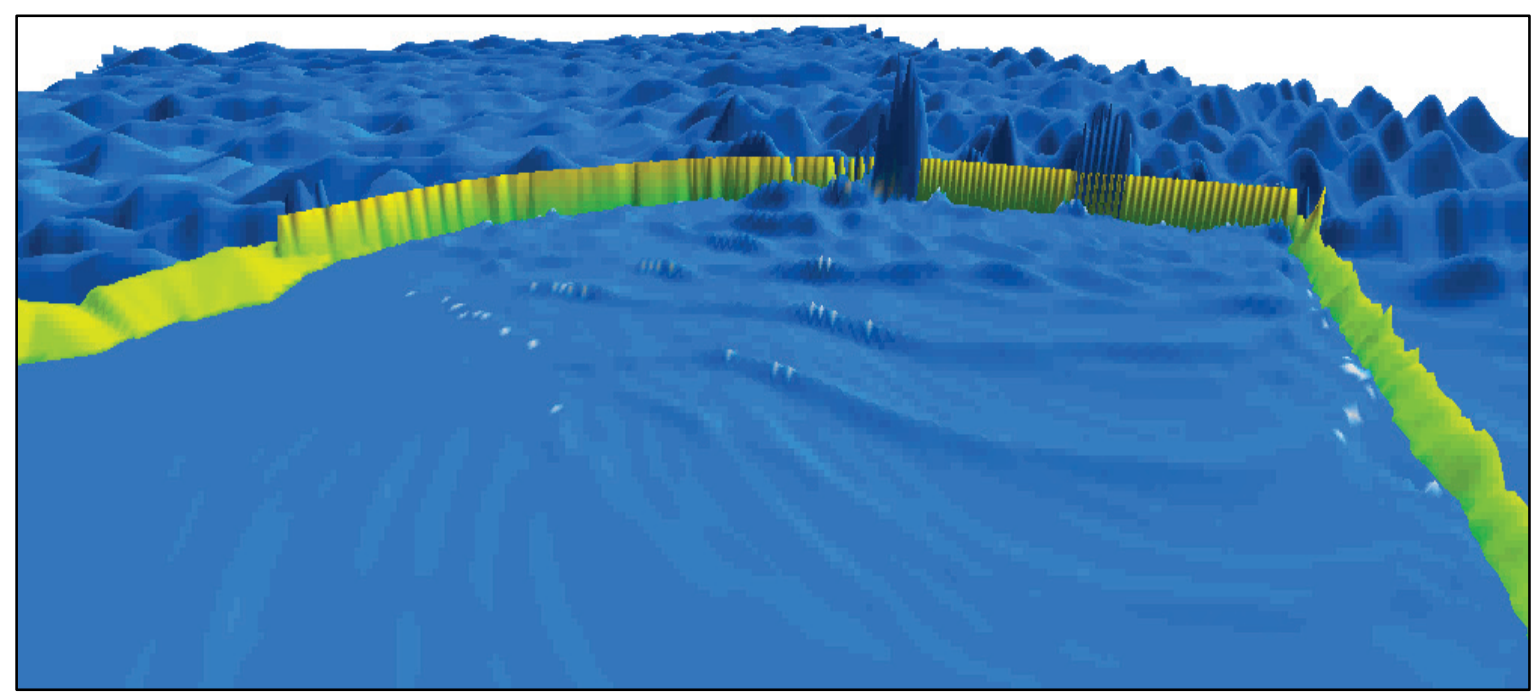


Figure 4-45. Overtopping at dike segments 3 and 5.

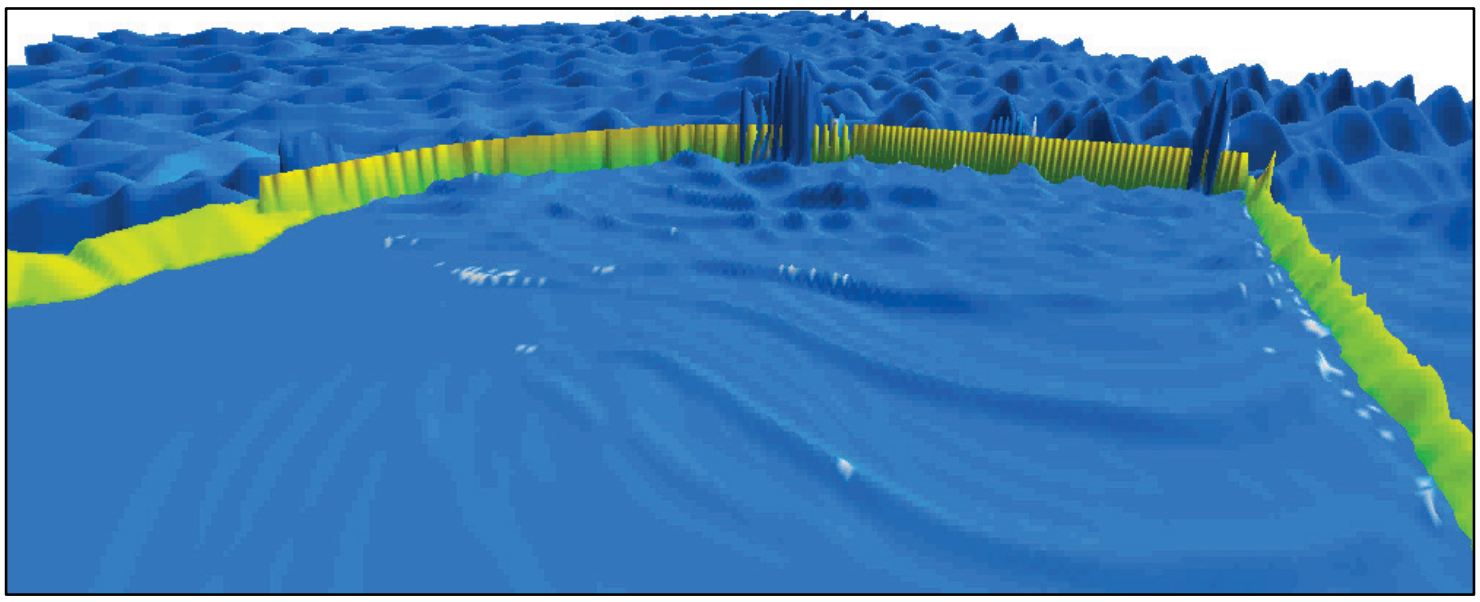

Figure 4-46. Overtopping at dike segments 2,3 , and 4 (scene 1 ).

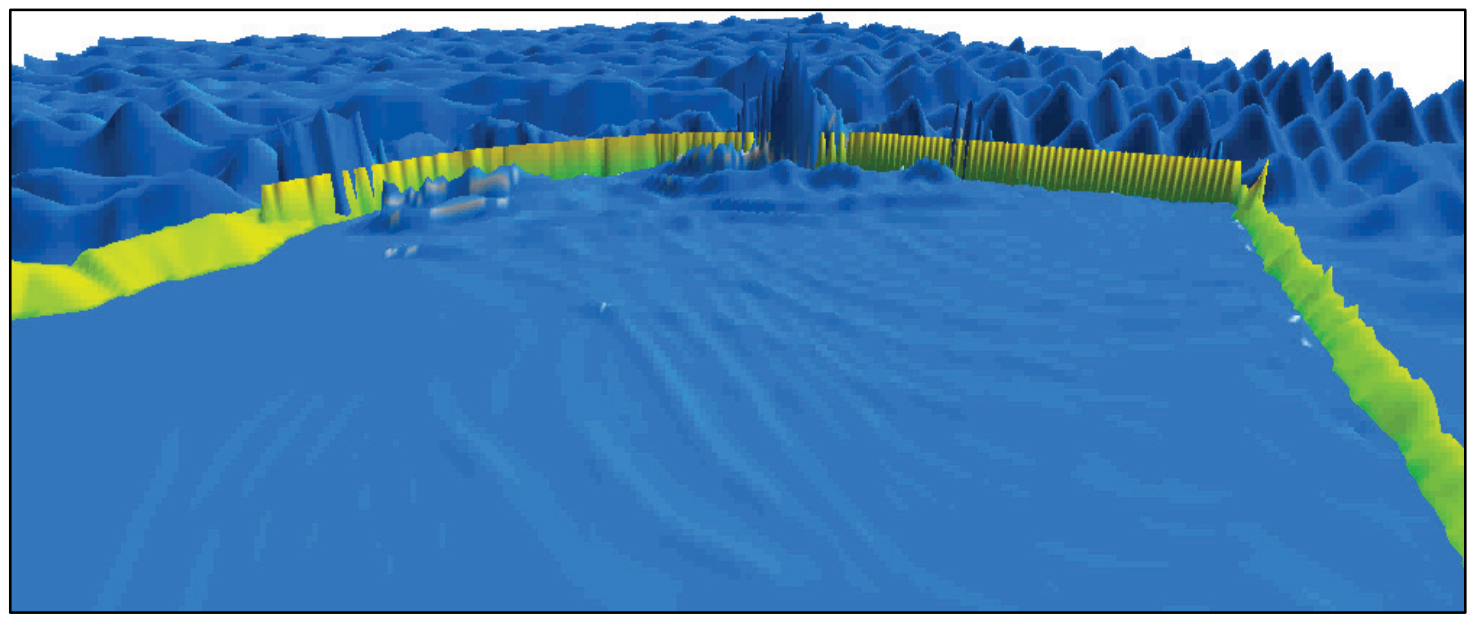

Figure 4-47. Overtopping at dike segments 2, 3, 4, and 5 (scene 2).

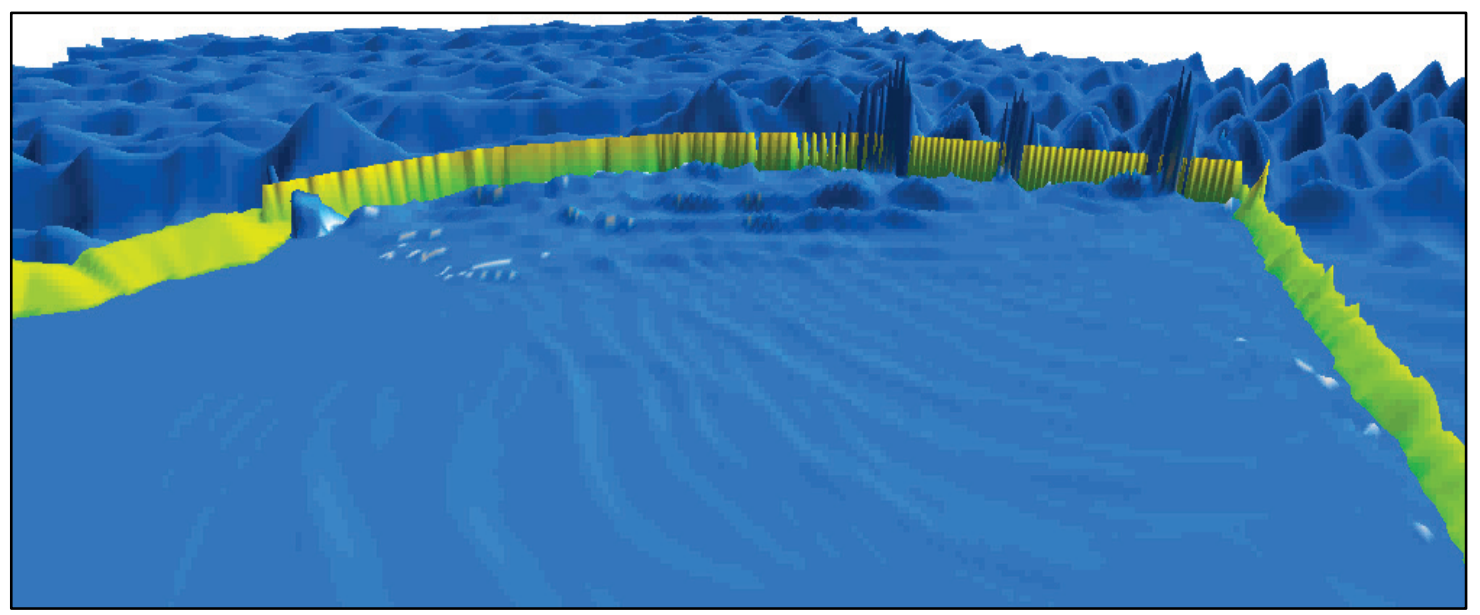


Figure 4-48. Overtopping at dike Segments 2, 3, and 4 (scene 2).

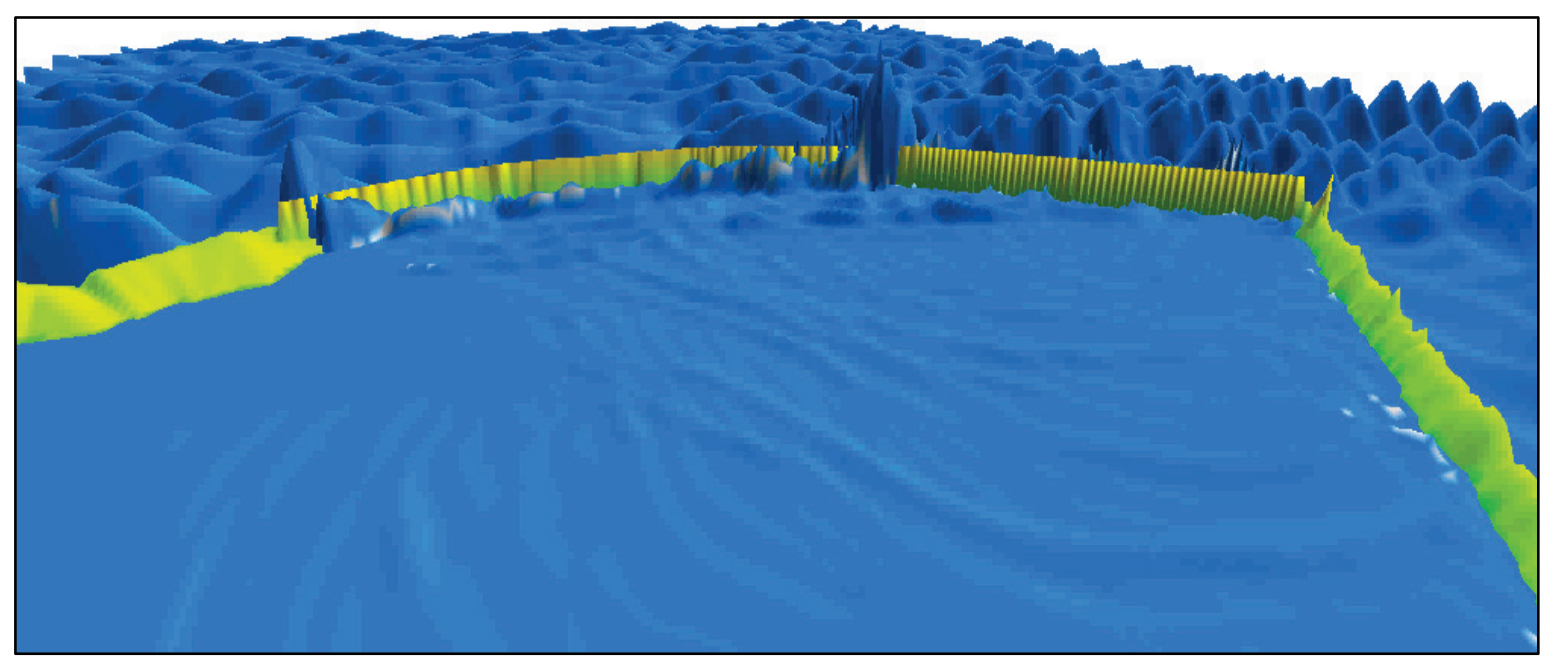

Figure 4-49. Overtopping at dike Segments 3, 4, and 5.

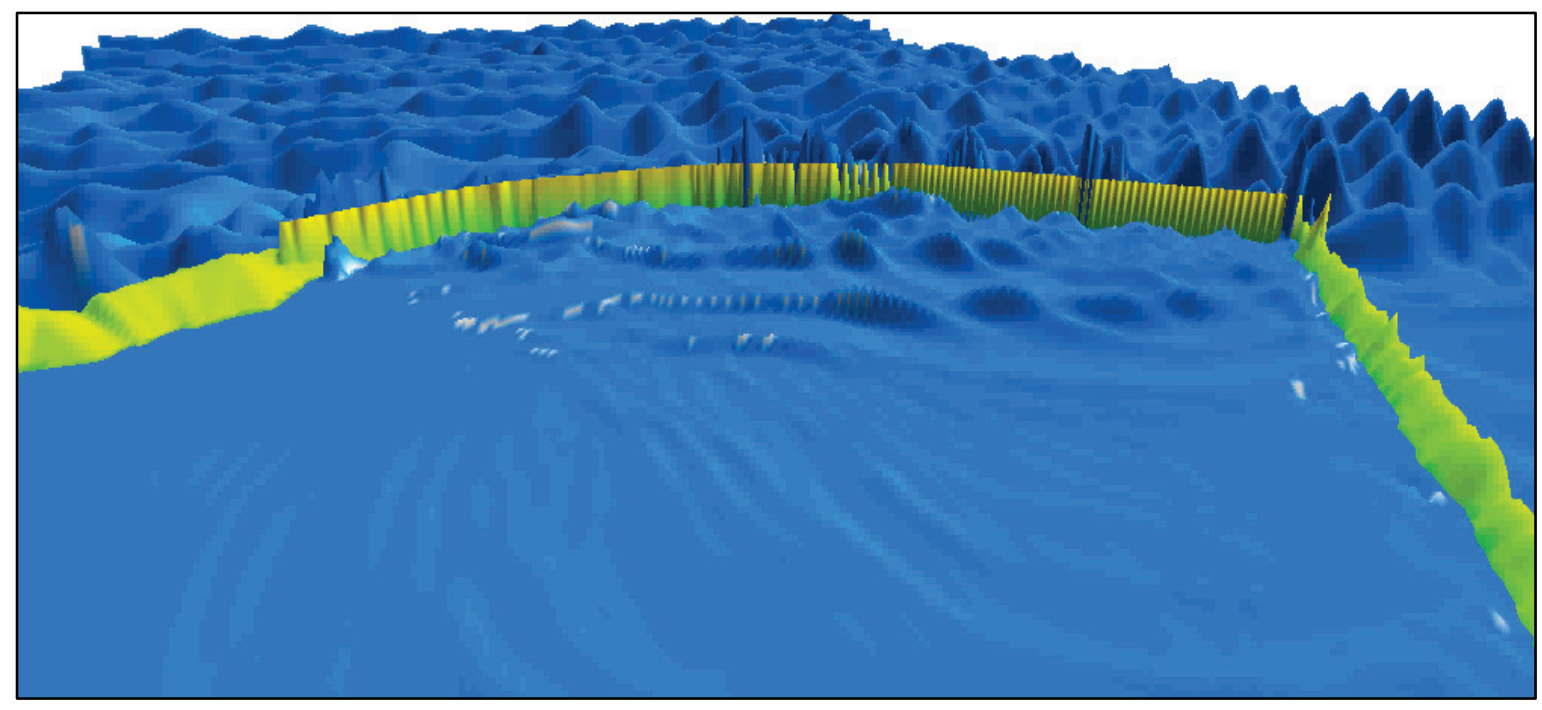

\subsubsection{Results for selected storm conditions}

Figures 4-50, 4-51, and 4-52 show wave heights simulated with B2D for Storm Conditions 1, 2, and 3, respectively. These figures present the spatial variation of model wave heights outside and inside the $\mathrm{CDF} 4$. Incident waves at the model offshore boundary for these storm simulations were $3.5 \mathrm{~m}, 4.0 \mathrm{~m}$

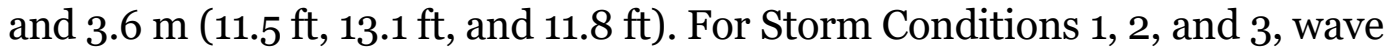
heights increased to $5.5 \mathrm{~m}, 5.8 \mathrm{~m}$, and $5.1 \mathrm{~m} \mathrm{(18.0} \mathrm{ft,} 19.0 \mathrm{ft}$ and $16.7 \mathrm{ft}$ ), respectively, near the lake side of Dike Legs 3, 4, and 5, where wave focusing occurs. Wave heights close to the structure are partly affected by wave reflection and diffraction from the dike but also influenced by the water level associated with the storm. Figure 4-53 shows the interior V-section area of the CDF4 with two selected arcs marked as A (black) and B (red) for 
examining the variation in wave and water level. The arcs were placed at the zero-elevation contour near the interior faces of the curved dike and the SEAB. This area is selected because it is directly adjacent to the wave focusing area along the lakeside Dike Legs 3, 4, and 5.

Figure 4-50. Wave height field of Storm Condition 1 (existing CDF4).

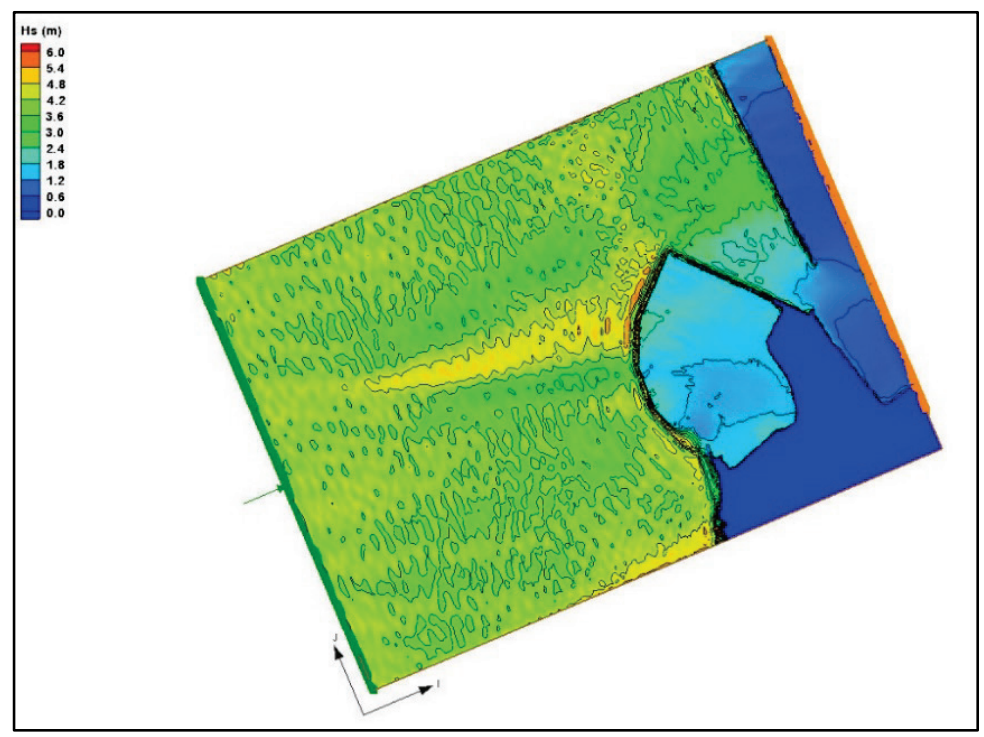

Figure 4-51. Wave height field of Storm Condition 2 (existing CDF4).

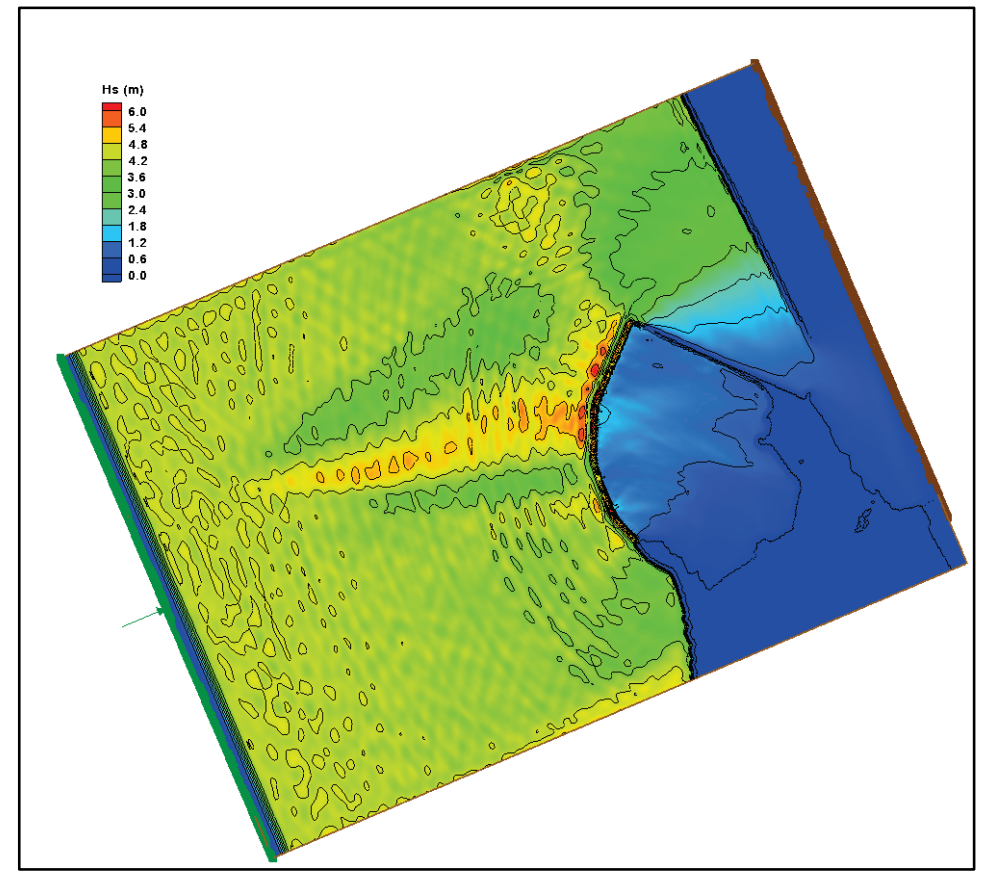


Figure 4-52. Wave height field of Storm Condition 3 (existing CDF4).

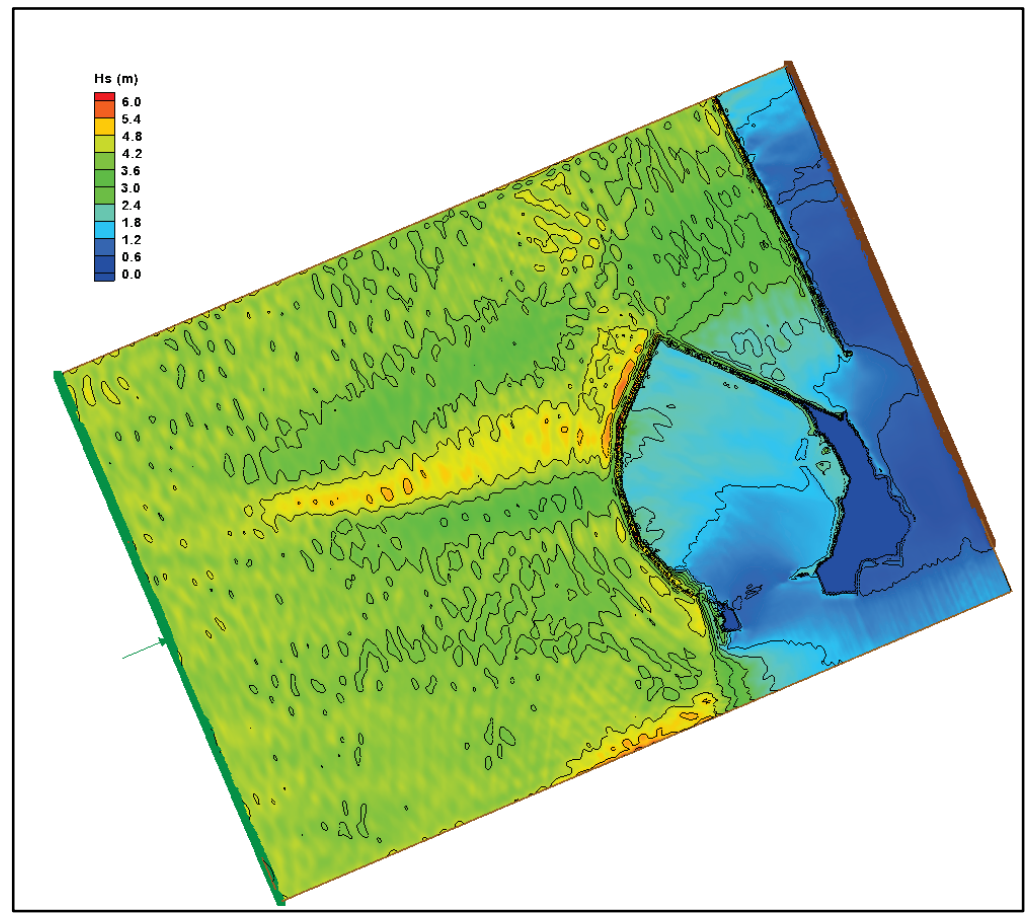

Figure 4-53. Arcs $A$ and $B$ in the interior V-section of CDF4.

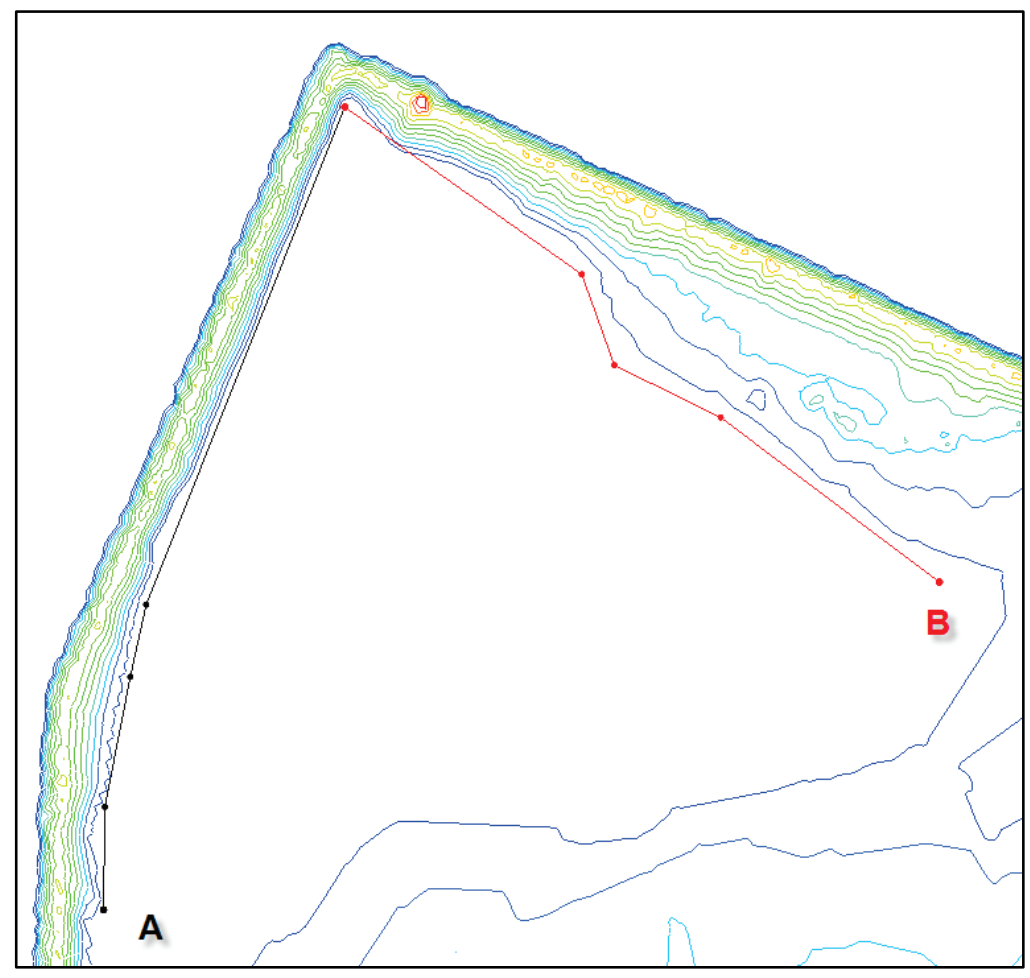

The variation in significant wave height (Hs) and mean water level (MWL) along the arcs A and B provides an estimate of waves inside the CDF4. Model results for Storm Condition 2 in Figure 4-54 indicate wave height and 
mean water level fluctuate in the $\mathrm{V}$-section of $\mathrm{CDF} 4$. Fluctuations up to 0,18 $\mathrm{m}$ in wave height and $0.4 \mathrm{~m}$ in water level along the selected interior arcs occur; larger fluctuations occur along arc A. These estimates suggest the overtopping of the SEAB would be unlikely given that the average crest elevation of the breakwater is greater than $4 \mathrm{~m}$. If long-duration storms and sustained overtopping of the dike occurs, a greater amount of flow would be entrapped inside $\mathrm{CDF} 4$ over a longer time period. With such a scenario, and also if wind effect was considered, wind waves in the interior and the elevated water level might cause water to exit over the SEAB. The majority of the $\mathrm{CDF}_{4}$ interior area is highly dissipative (e.g., covered with vegetation and debris), and the area open to wave growth is limited. In addition, the breakwater and curved dike are expected to be slightly porous, releasing trapped water. For these reasons, a high rise in water level inside the $\mathrm{CDF} 4$ might not occur; therefore, waves inside the CDF4 would not reach significant heights and overtop the breakwater on the entrance channel side. Last, there is a large variation in heights of the entrapped waves and water levels within the $\mathrm{V}$-section of the $\mathrm{CDF}_{4}$ in time and space as waves are piling up and sloshing between the right and left sides of the $\mathrm{V}$-section. Consequently, wave height and water level exhibit a different pattern along arcs A and B and do not match where the arcs come closer.

Figure 4-54. Hs and MWL along arcs A and B for Storm Condition 2 (existing dike).

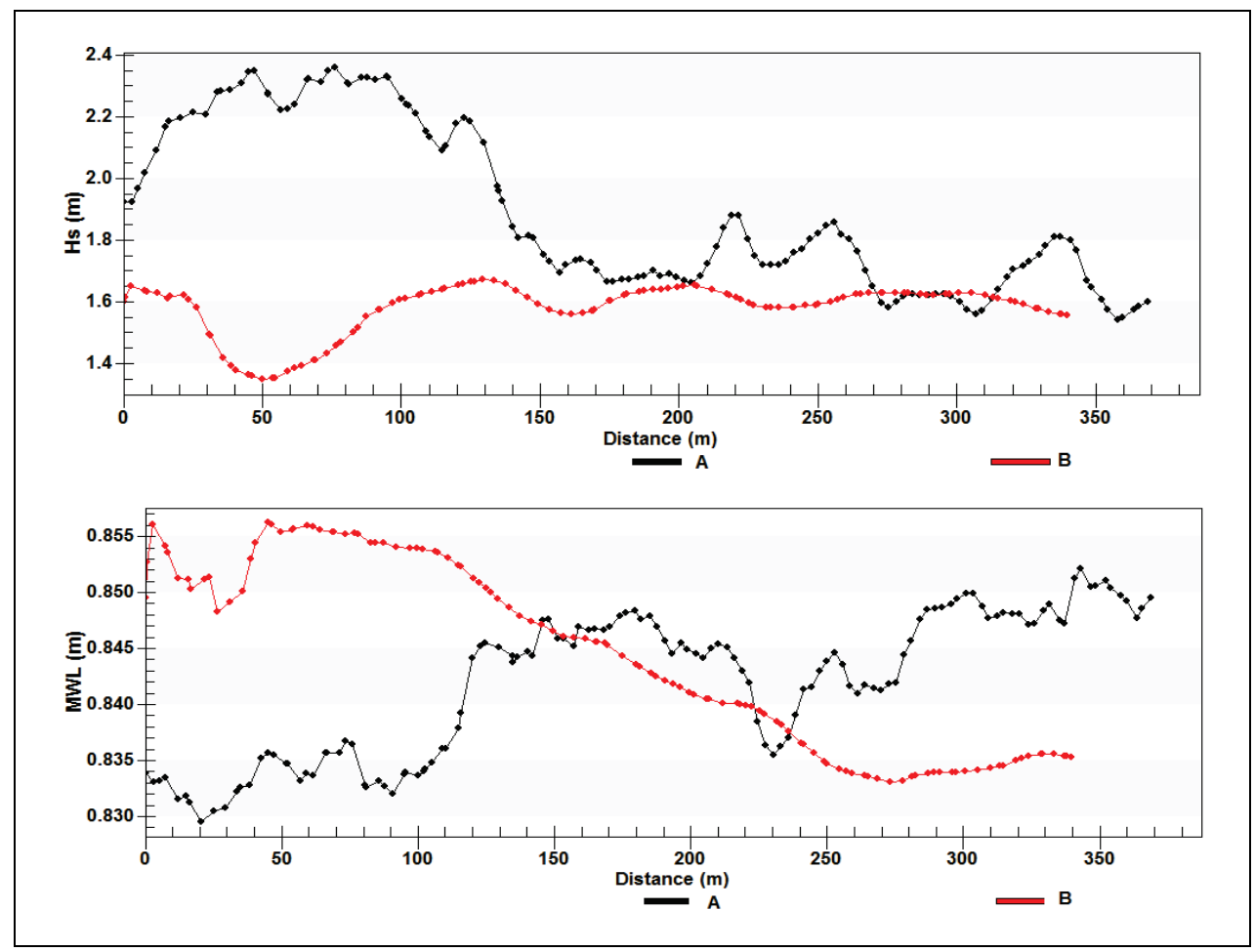




\subsubsection{Estimates of overtopping}

Estimates of wave overtopping for the existing CDF4, based on a coupled $\mathrm{B} 2 \mathrm{D}$ and $\mathrm{B} 1 \mathrm{D}$ modeling approach, are described in this section. The 2D Boussinesq modeling (B2D) results showed that wave overtopping occurred at various segments of the curved dike at different locations and times. The sensitivity tests conducted using different $2 \mathrm{D}$ grids indicated that detailed features of wave runup and overtopping over the dike were sensitive to the grid resolution. For computational efficiency, a $4 \mathrm{~m}$ grid resolution was used in B2D modeling while tests suggested that grid resolution on the order of $1 \mathrm{~m}$ was necessary to adequately resolve Wave runup and overtopping. However, 2D Boussinesq modeling with a $1 \mathrm{~m}$ resolution would be computationally infeasible. This led to consideration of using a $1 \mathrm{D}$ Boussinesq model (B1D) capable of resolving necessary details of the wavedike interaction processes, including the wave runup and overtopping.

The application of $\mathrm{B} 2 \mathrm{D}$ to drive a one-spatial-dimension (B1D) model is common in some engineering practice (Demirbilek et al. 2007a,b, 2005a,b; Nwogu and Demirbilek 2001). B1D allows for the use of the finest grid resolution necessary for a given application while taking advantage of the nonlinear capabilities of the Boussinesq model. In this approach, the model uses a $1 \mathrm{D}$ spatial grid or transect. In the present application, waves propagate in the $x$-direction with the associated elevation in the $z$-direction. These $(x, z)$ pairs of bathymetry are sometimes referred to as the "transect profile" or "line profile" or simply as the "profile." B1D modeling ensures improved accuracy of wave overtopping estimates for the curved dike of CDF4 along the identified ten transects. Note that in adopting the B1D modeling approach, each transect profile must be continuous. A transect can consist of curved pieces (it is not required to be a straight line).

The $\mathrm{B} 1 \mathrm{D}$ simulations used the B2D modeling results extracted at a specified location in the lake near the structure as a forcing condition. The CEM and Eurotop manuals recommend that the forcing location be at a distance that is near to the toe of structure that could extend to approximately one wavelength from the structure's toe. If necessary, the shorter transect profiles can be extended to the specified distance to define the starting ends of each transect. The Arc3 in Figure 4-55 defines the location of B1D inputs (wavemaker) for ten transects used in this study, approximately $150 \mathrm{~m}$ (492

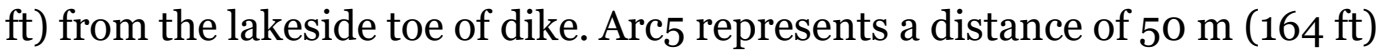
from the toe, Arc1 the mid-section of structure (crest location), and Arc2 the back-side (interior) toe. The transects were extended to the Arc3, and $(x, z)$ bathymetry files for each transect were constructed for the B1D model. 
Figure 4-55. Coupled B2D and B1D modeling using Arc3 as the wavemaker location for B1D.

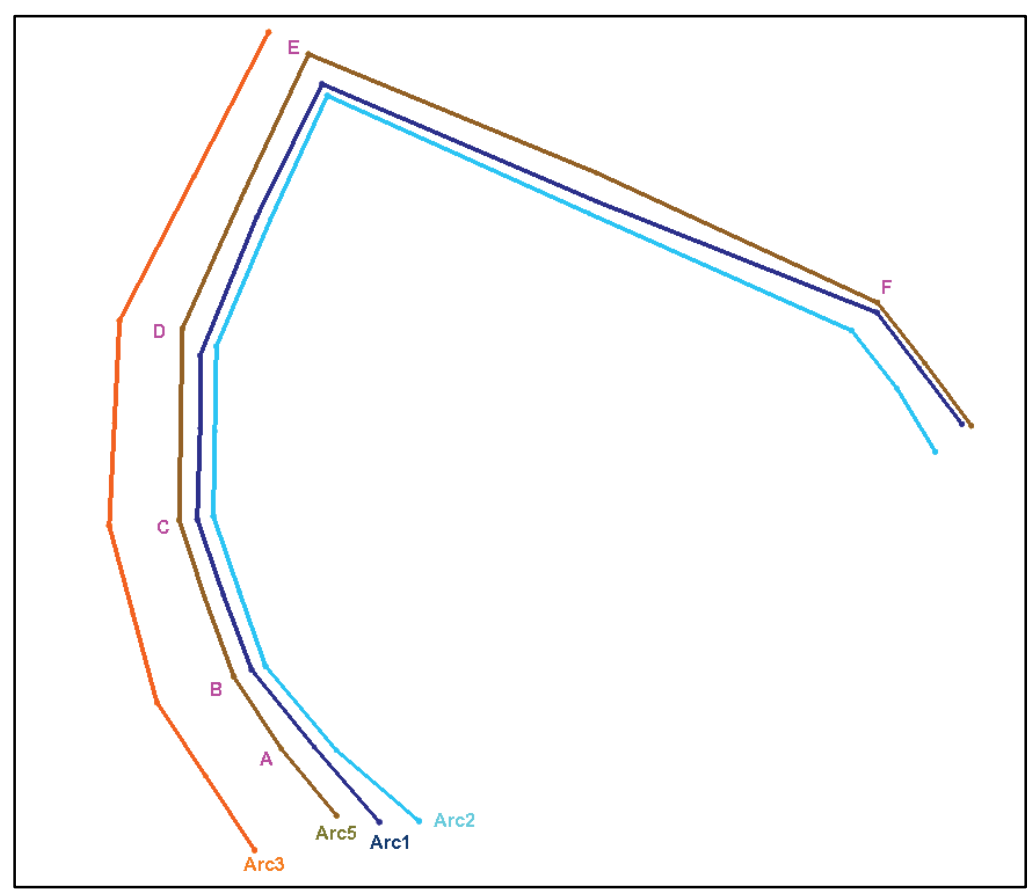

Test runs were conducted with the B1D grid spacing (delx) of 0.5, 1.0, 1.5, 2.0, and $2.5 \mathrm{~m}(1.6,3.3,4.9,6.6$, and $8.2 \mathrm{ft})$, and delx $=1 \mathrm{~m}(3.3 \mathrm{ft})$ was selected for the production runs based on guidance provided by Nwogu and Demirbilek(2001). The previously discussed B2D model inputs and stability issues apply to the $\mathrm{B} 1 \mathrm{D}$ modeling. The controlling parameters such as appropriate values of the time-step, damping layers for the up- and downwave ends of the transect profiles, and values of the Chezy, Smagorinsky, and turbulent length scale were determined from a series of B1D test runs. $B 1 D$ results were saved at the delx-spaced grid points probes and special probes (gauges) placed along each transect profile. Model results were analyzed in the time domain to develop estimates of wave height, wave runup, and overtopping of the dike along each transect's profile.

Both time series and integrated estimates (e.g., hydrographs of wave runup and overtopping) were developed to provide spatial and temporal changes in these engineering quantities of interest. Model results for Storm Condition 2 , the most intense storm condition, are presented to minimize or eliminate excessive overtopping of the dike and its potential consequences for any future remedial actions. A few samples for other less severe storm conditions are provided. The results in Figures 4-26 to 4-49 indicated dike segments in the middle and north experienced the most severe overtopping. Model results are provided for three representative transects (T2, T5, and 
T9) for the south (Leg 2), middle (intersection of Legs 3 and 4), and north (Leg 5) segments of the curved dike. Results for other transects are provided in Appendix D.

Figures 4-56 through 4-64 show results for transects T2, T5, and T9 including the wave height along each transect, transect cross section, and temporal variation in wave runup and overtopping rate. Engineering estimates were developed by analysis of the spatial and temporal records (hydrographs) and summaries are provided in Tables 4-2, 4-3, and 4-4 for all ten transects.

Figure 4-56. Wave height variation along transect T2.

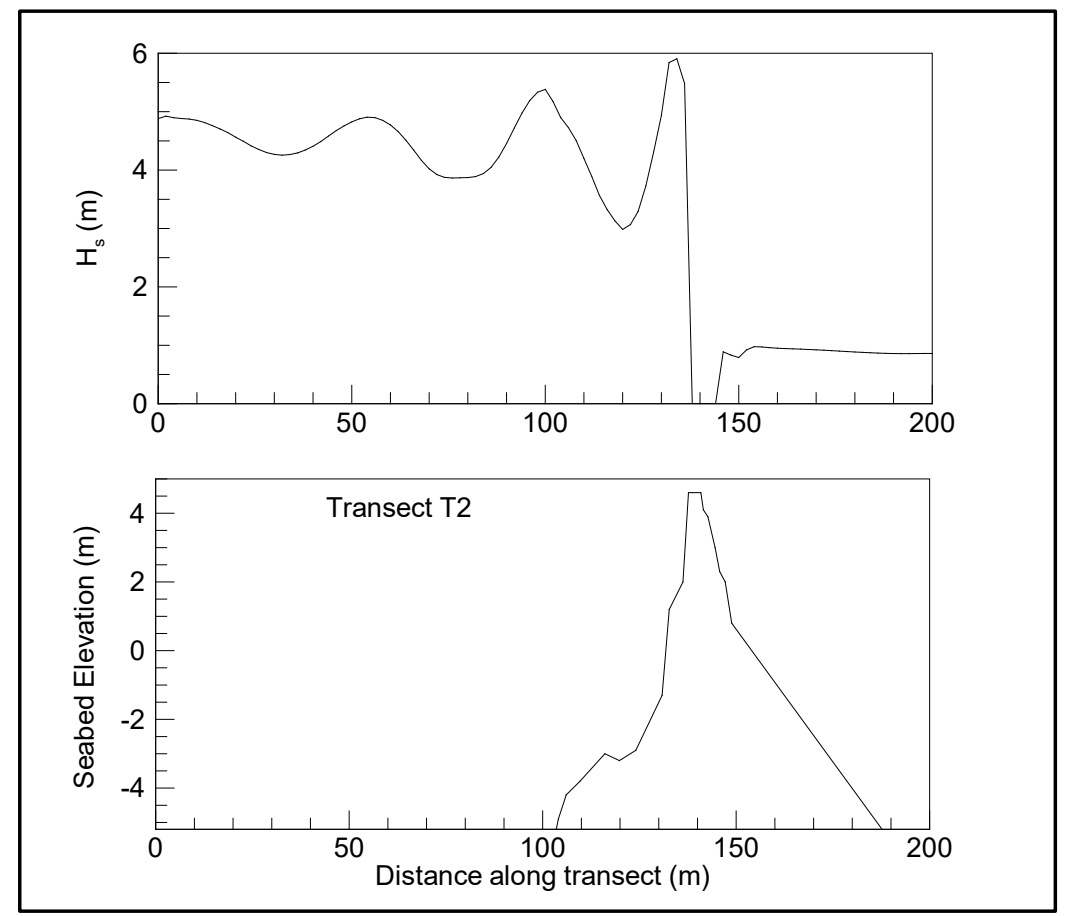

Furthermore, for the overtopping rates shown in Figures 4-57 and 4-58, which cover a time span of 1,000 and 4,000 sec, respectively, the time period is centered on the peak of each storm. 
Figure 4-57. Overtopping rate and runup height variation along transect T2 (first 1,000 sec).

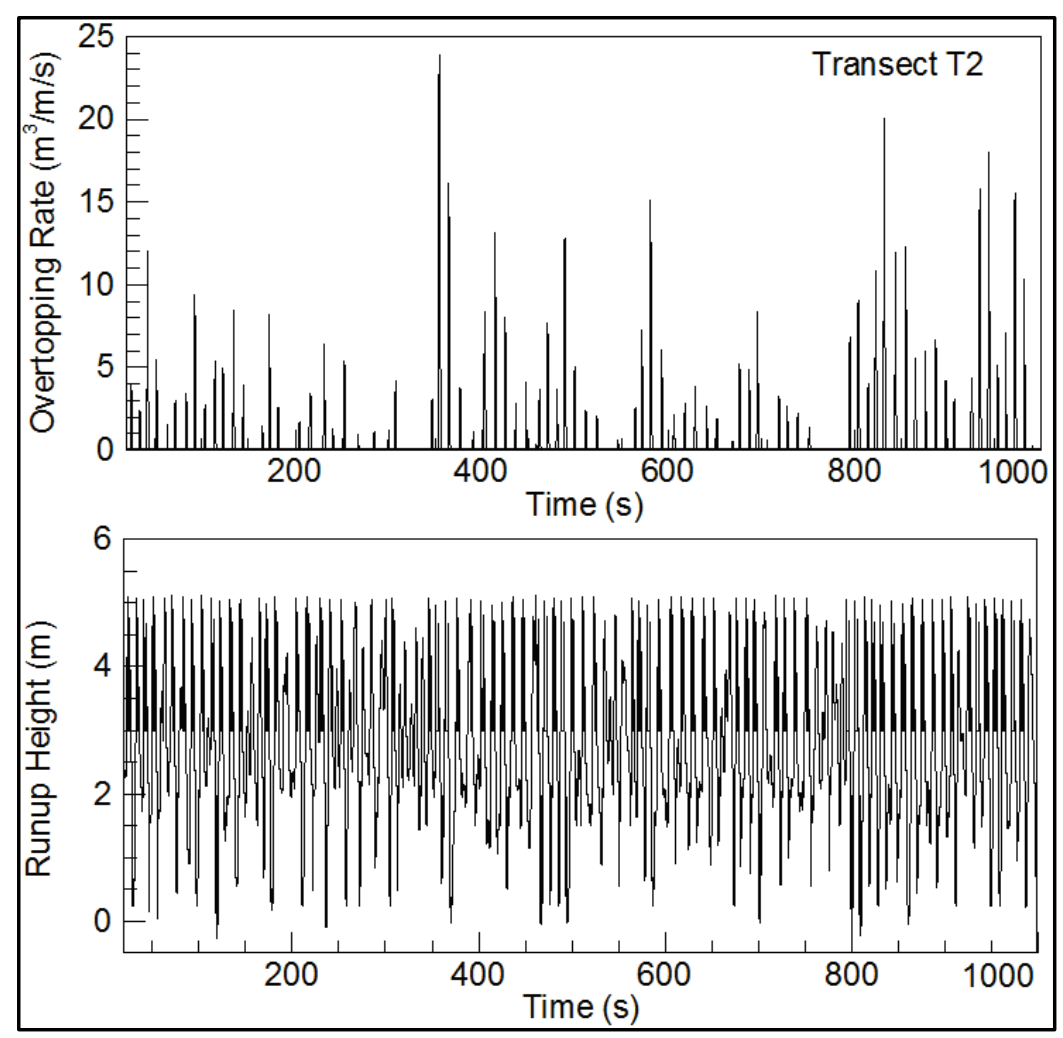

Figure 4-58. Time variation of overtopping rate along transect T2 (entire simulation).

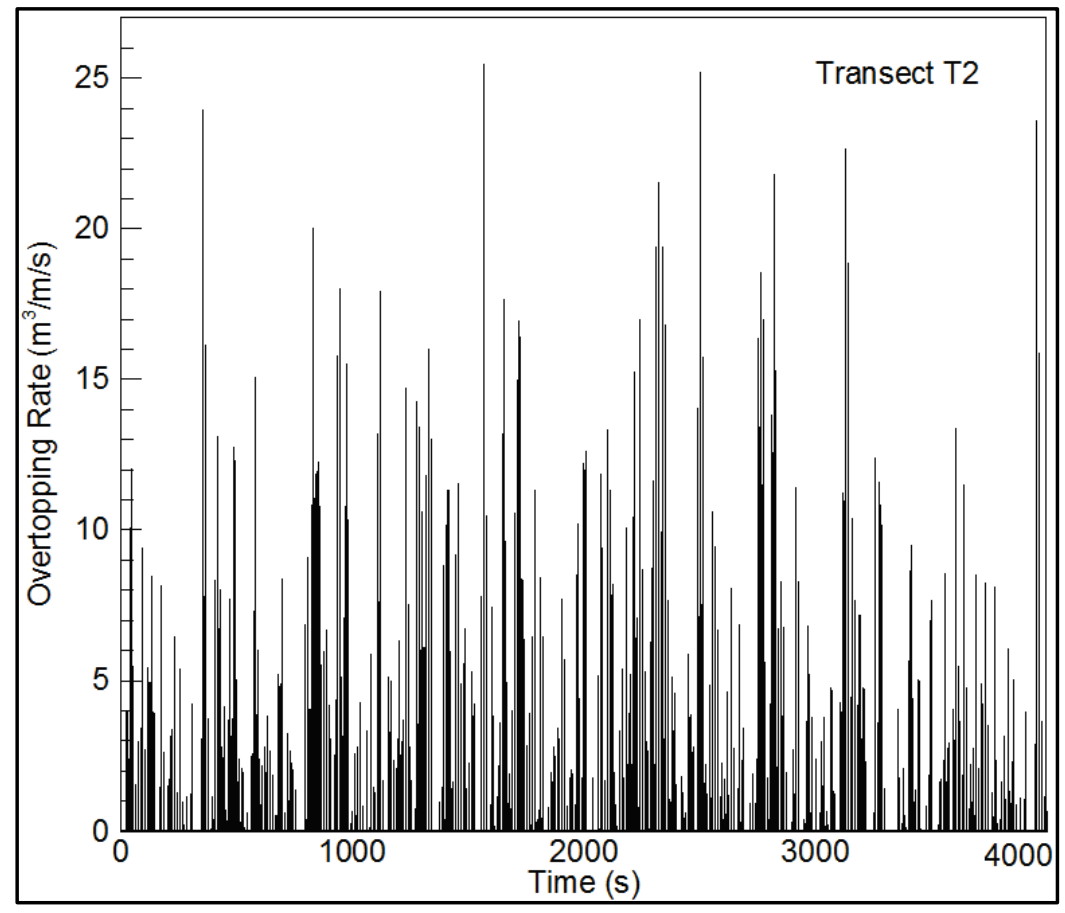


Figure 4-59. Variation of wave height along transect T5.

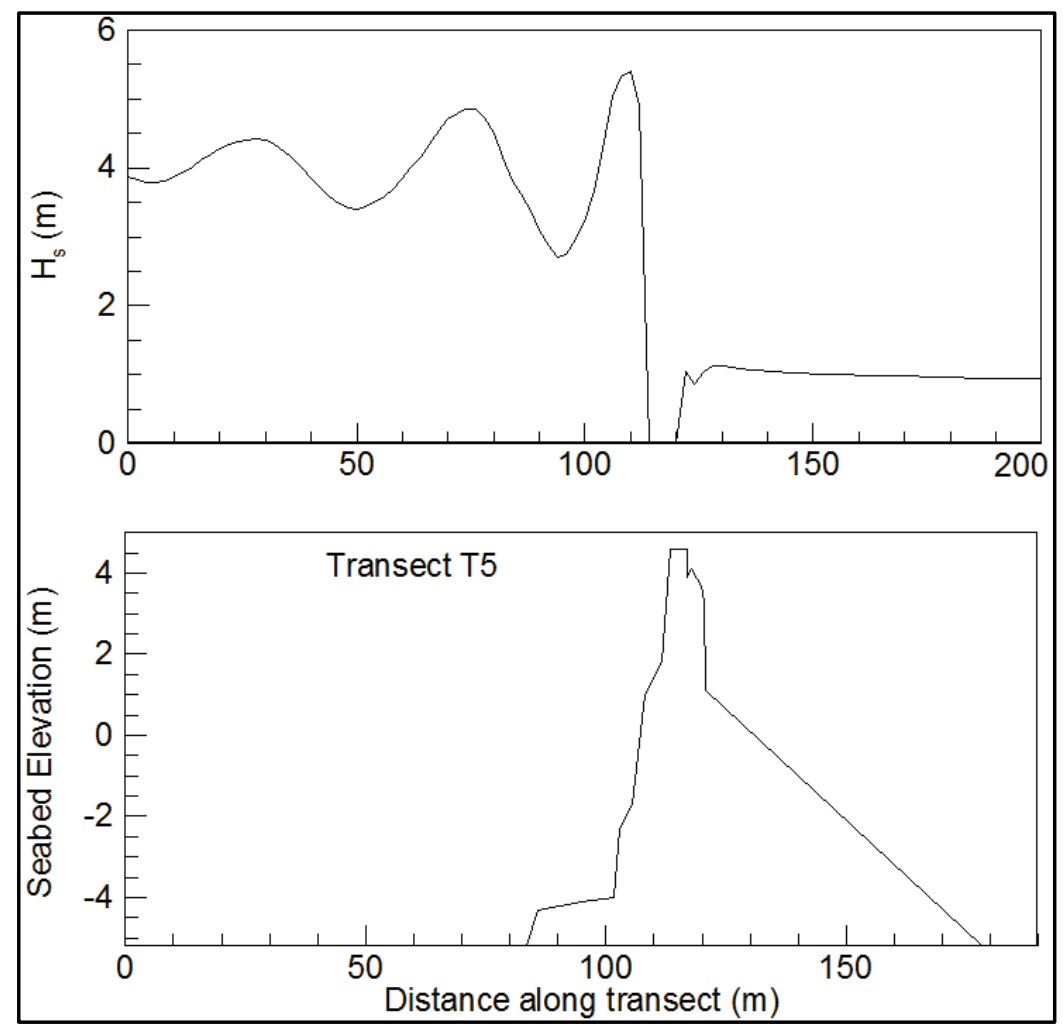

Figure 4-60. Variation of overtopping rate and runup height along transect T5 (first 1,000 sec).

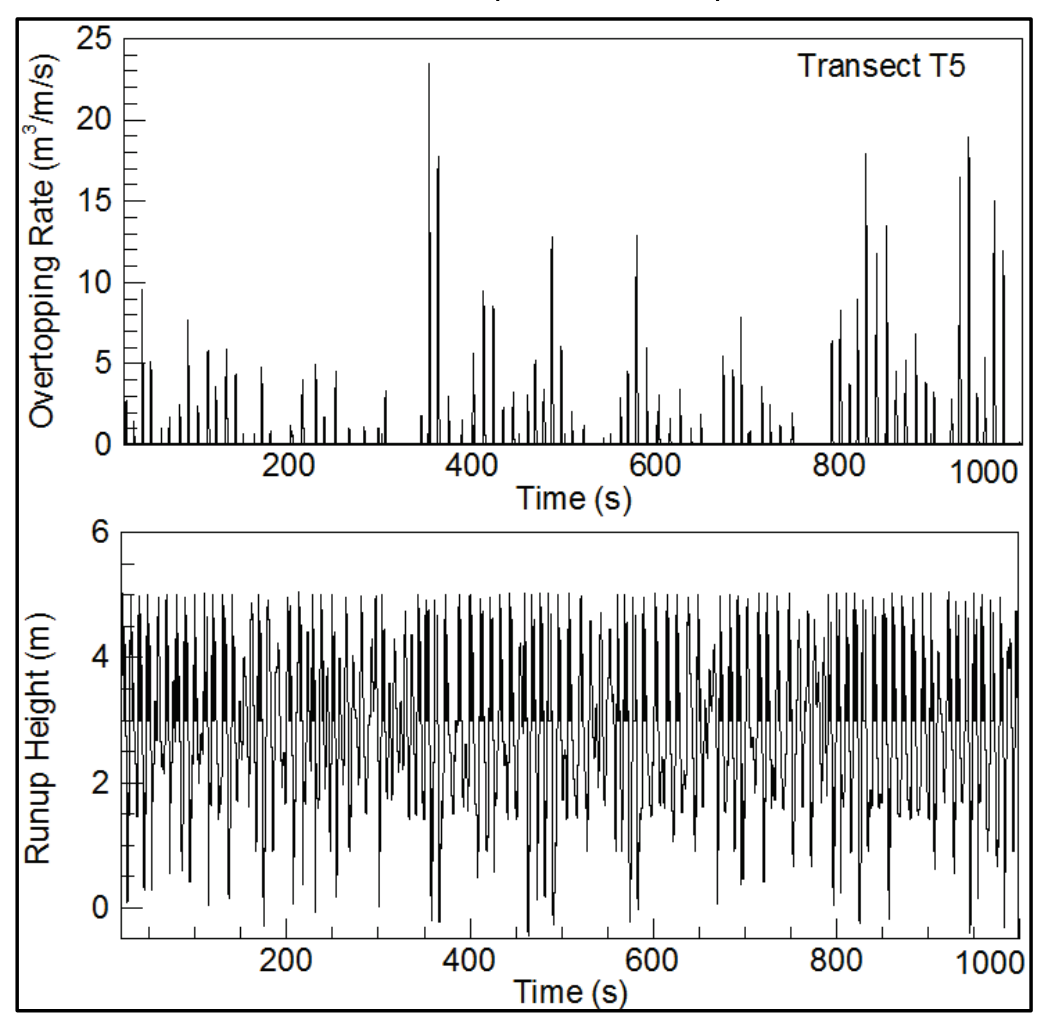


Figure 4-61. Time variation of overtopping rate along transect T5 (entire simulation).

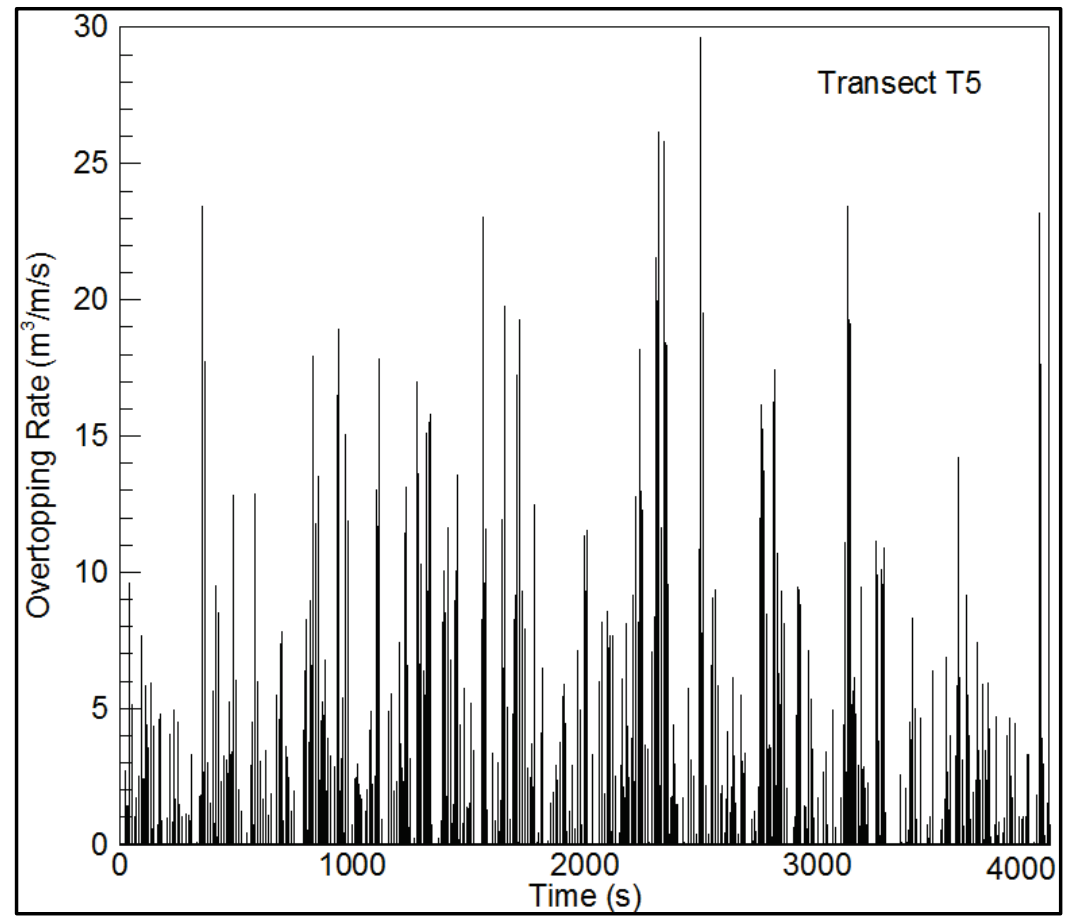

Figure 4-62. Wave height variation along transect T9.

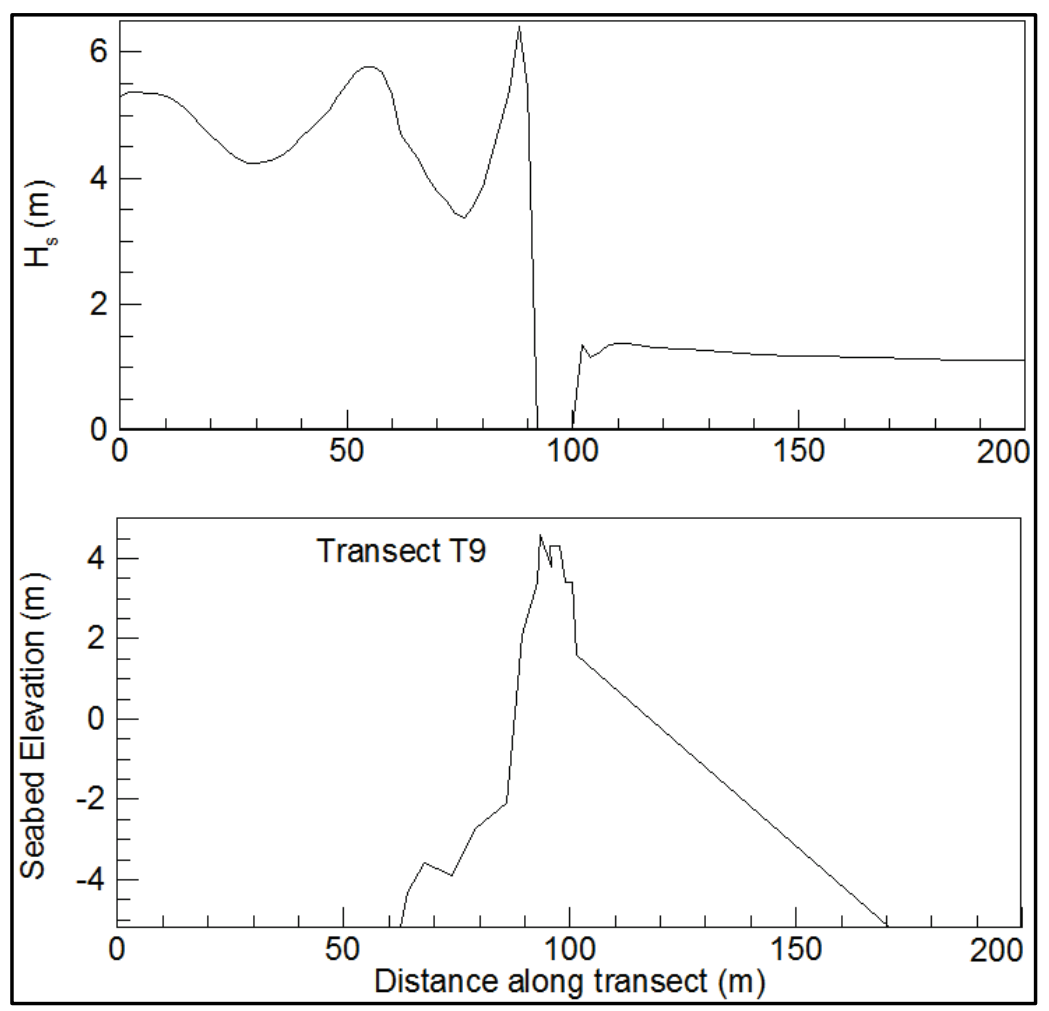


Figure 4-63. Overtopping rate and runup height variation along transect T9 (first 1,000 sec).
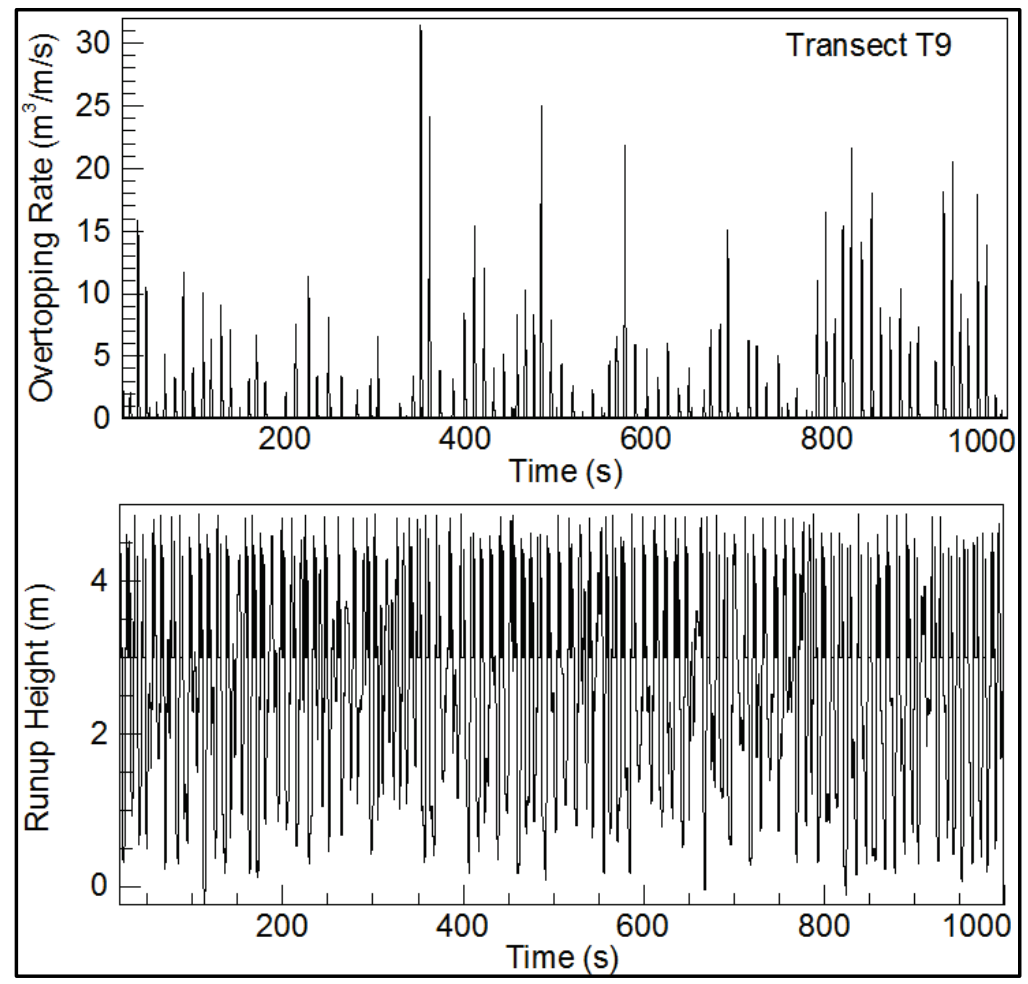

Figure 4-64. Time variation of overtopping rate along transect T9 (entire simulation).

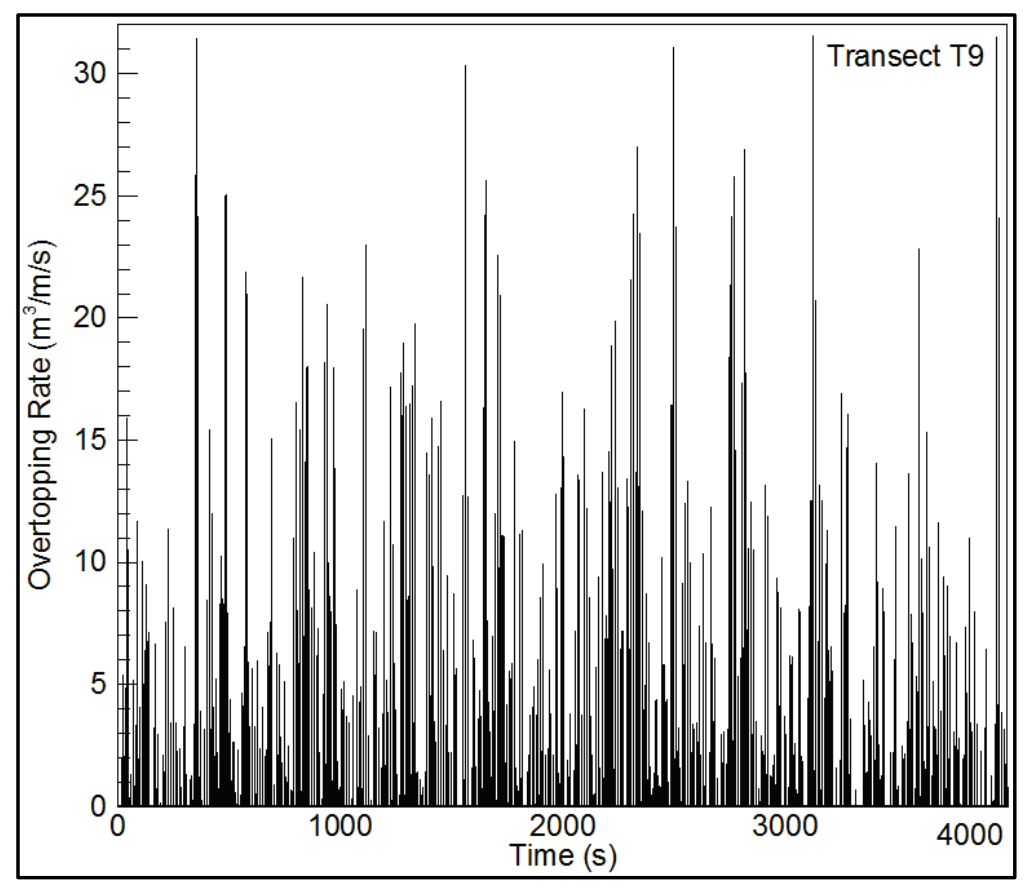


Time series or hydrographs of the overtopping rates (Figures 4-56 through 4-64) were analyzed to develop statistical estimates of parameters of engineering interest. These included minimum, average, maximum, standard deviation, and RMS (root-mean-square) values of the time series saved at each grid point on each transect profile. In practical applications, the average overtopping is the primary estimate of interest. For Storm Condition 2, the average overtopping rates are provided in Figure 4-65 for all transects. Results for Storm Conditions 1 and 3 are also provided in Figures 4-66 and 4-67, respectively.

The wave height and overtopping statistics were obtained by analyzing calculated time series data from B1D simulations along Transects T1 through T10 for the strongest condition simulated (Storm Condition 2). The record length of each time series was 4,200 sec and had 208,990 samples. For Storm Condition 2, average and maximum statistics used in engineering works are provided in Table 4-2. The time-consuming and tedious analysis is necessary for developing design and repair estimates for engineering studies. This time-domain analysis may also be used to develop a variety of first-order statistics of other model results generated by B1D/B2D. Such optional model results available for analysis include wave runup, wave pressure, wave-induced current, and wave forces.

Figure 4-65. Average overtopping rate for transects T1 to T10 (Storm Condition 2).

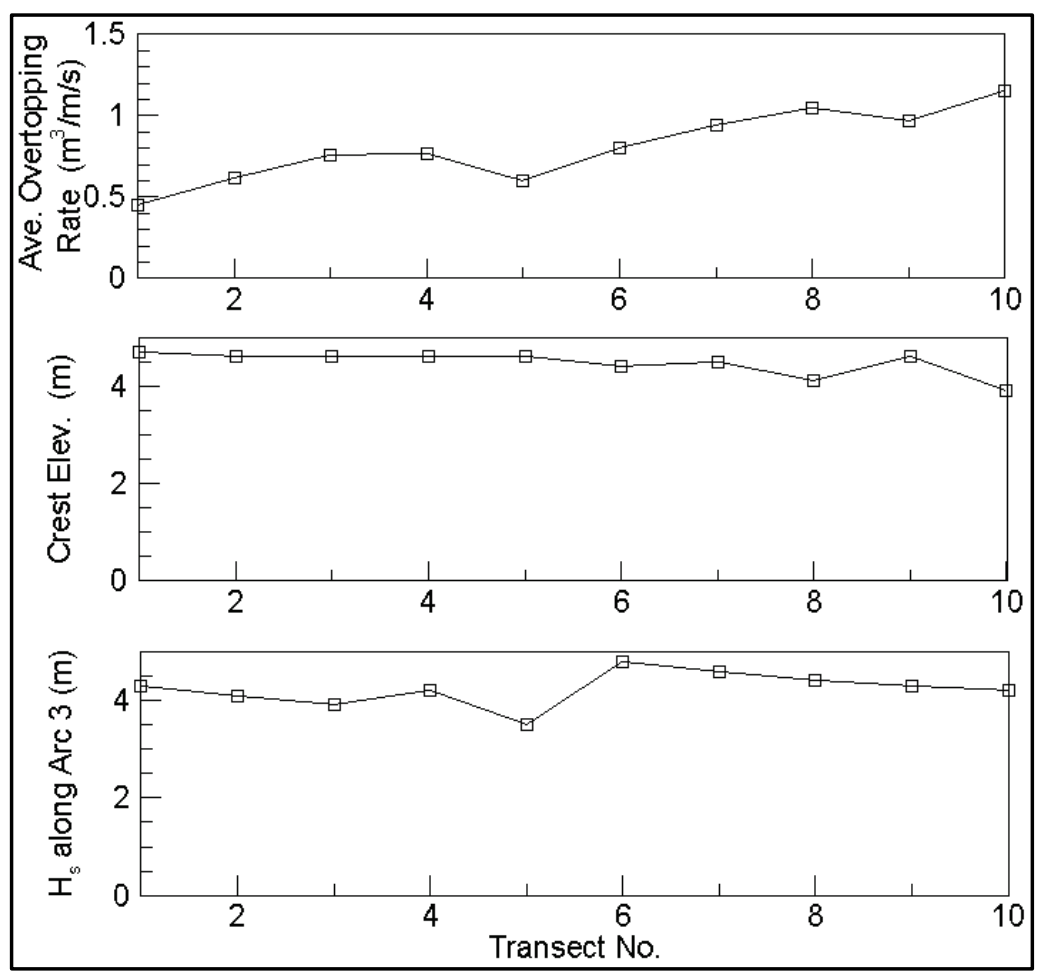


Figure 4-66. Average overtopping rate for transects T1 to T10 (Storm Condition 1).

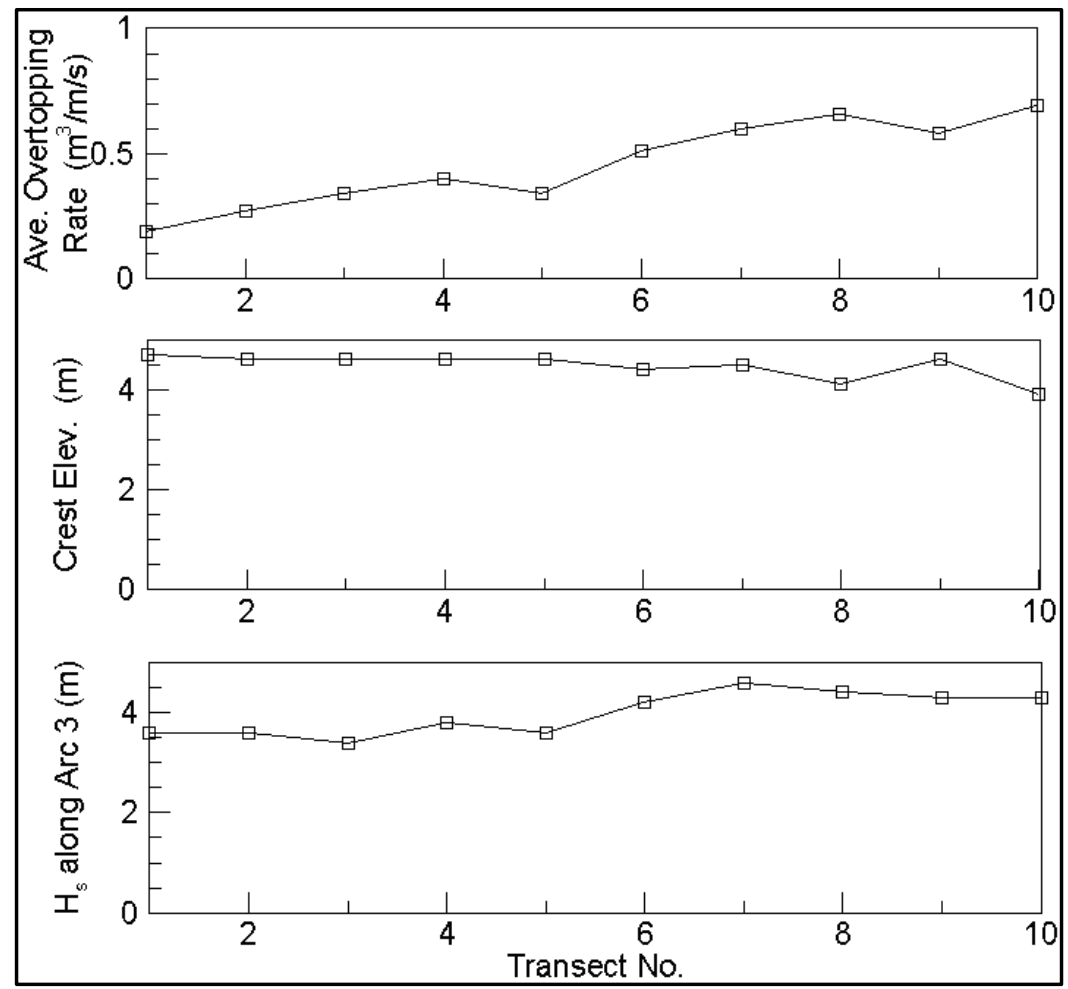

Figure 4-67. Average overtopping rate for transects T1 to T10 (Storm Condition 3).

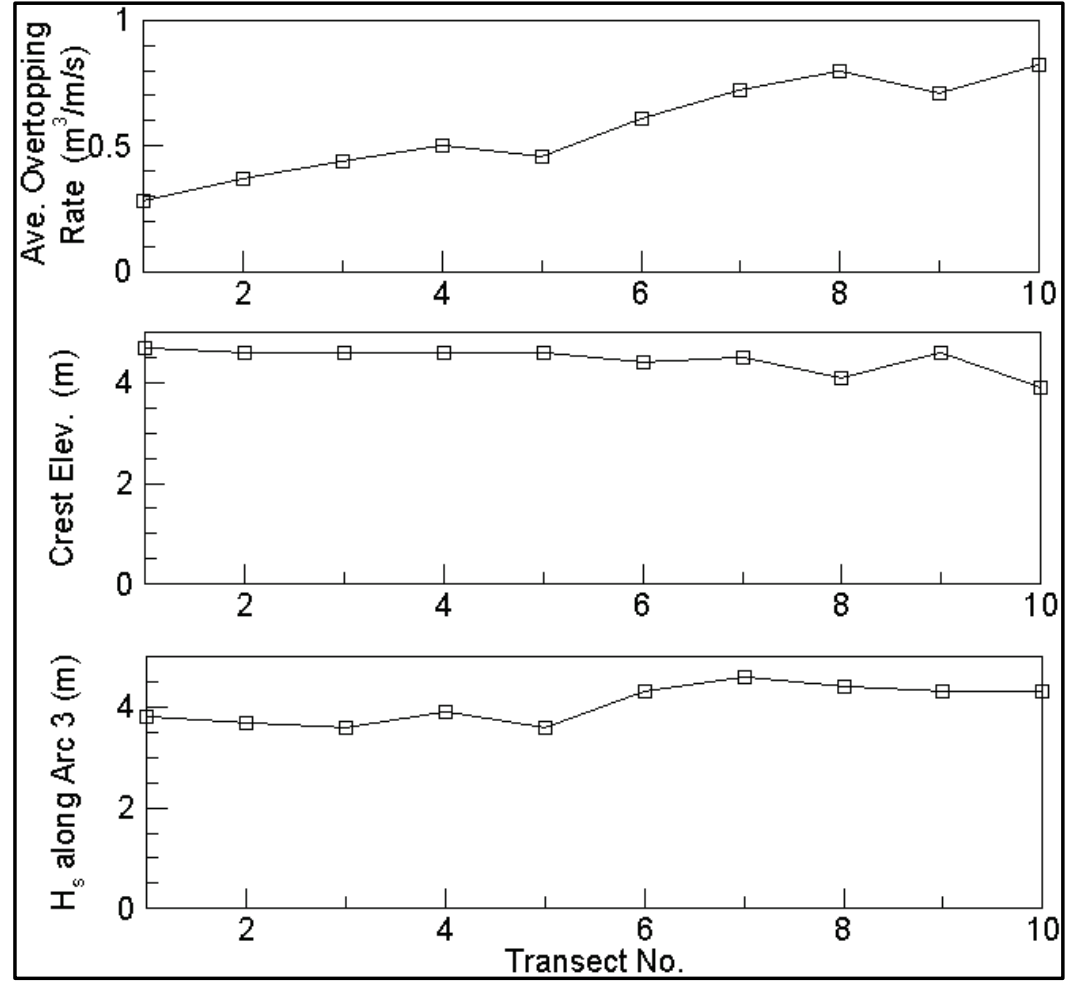


Table 4-2. Wave height and overtopping rate statistics for transects T1 through T10 (Storm Condition 2).

\begin{tabular}{|c|c|c|c|c|}
\hline \multirow{2}{*}{ Transect } & \multicolumn{2}{|c|}{$\begin{array}{c}\text { Hs } \\
\text { Overtopping rate }(\mathrm{m})\end{array}$} & \multicolumn{2}{c|}{ (s) } \\
\cline { 2 - 5 } & Average & Maximum & Average & Maximum \\
\hline T1 & 2.38 & 5.67 & 0.43 & 21.06 \\
\hline T2 & 2.54 & 5.91 & 0.59 & 25.47 \\
\hline T3 & 2.32 & 5.22 & 0.74 & 27.39 \\
\hline T4 & 2.64 & 5.89 & 0.74 & 28.74 \\
\hline T5 & 2.23 & 5.40 & 0.57 & 29.62 \\
\hline T6 & 2.60 & 6.14 & 0.95 & 30.36 \\
\hline T7 & 2.54 & 6.65 & 0.92 & 29.25 \\
\hline T8 & 2.52 & 6.14 & 1.03 & 28.67 \\
\hline T9 & 2.65 & 6.41 & 0.95 & 31.55 \\
\hline T10 & 2.63 & 5.98 & 1.12 & 41.62 \\
\hline
\end{tabular}

Wave and overtopping rate statistics from the Storm Conditions 1 and 3 simulations are presented for completeness and comparison of the three storm conditions. Table 4-3 presents statistics of wave height and overtopping rate for Storm Condition 1. Table 4-4 provides the statistics for Storm Condition 3. Comparison of statistics for the three storms show some minor differences between storm results even though wave parameters and water levels used in their simulations were comparable (Table 4-1). Incident wave periods and directions for the three storms were nearly identical (within +/-1 deg); however, the maximum difference in input water levels was $0.7 \mathrm{~m}$. The range for average overtopping rate is 0.2 to $1.2 \mathrm{~m}^{2} / \mathrm{s}$ while maximum overtopping rate is between 20 to $40 \mathrm{~m}^{2} / \mathrm{s}$. Wave height and overtopping are consistently higher for transects T6 through T10 for all three simulated storms.

It is interesting to compare these estimates to those by the CMS or the Eurotop manual presented in Chapter 3. Although two different wave models use different calculation methods for estimates of wave overtopping, the average overtopping rates from $\mathrm{B} 2 \mathrm{D} / \mathrm{B} 1 \mathrm{D}$ are comparable to the mean of the peak overtopping rates by the CMS for permeable structures. B2D/B1D simulations were centered on the peak duration of storms during which storm water levels were assumed to remain constant (not changing). 
Table 4-3. Wave height and overtopping rate statistics for transects T1 through T10 (Storm Condition 1).

\begin{tabular}{|c|c|c|c|c|}
\hline \multirow{2}{*}{ Transect } & \multicolumn{2}{|c|}{$\begin{array}{c}\text { Hs } \\
\text { Overtopping rate }(\mathrm{m})\end{array}$ **2/s) } \\
\cline { 2 - 5 } & Average & Maximum & Average & Maximum \\
\hline T1 & 2.27 & 4.90 & 0.19 & 14.37 \\
\hline T2 & 2.36 & 5.33 & 0.27 & 18.91 \\
\hline T3 & 2.52 & 5.38 & 0.37 & 22.32 \\
\hline T4 & 2.70 & 5.83 & 0.44 & 24.81 \\
\hline T5 & 2.27 & 5.25 & 0.37 & 24.97 \\
\hline T6 & 2.50 & 5.85 & 0.54 & 25.47 \\
\hline T7 & 2.63 & 6.89 & 0.61 & 24.52 \\
\hline T8 & 2.61 & 6.74 & 0.67 & 27.11 \\
\hline T9 & 2.48 & 6.71 & 0.60 & 25.97 \\
\hline T10 & 2.46 & 6.01 & 0.67 & 35.41 \\
\hline
\end{tabular}

Table 4-4. Wave height and overtopping rate statistics for transects T1 through T10 (Storm Condition 3).

\begin{tabular}{|c|c|c|c|c|}
\hline \multirow{2}{*}{ Transect } & \multicolumn{2}{|c|}{$\begin{array}{c}\text { Hs } \\
\text { Overtopping rate }(\mathrm{m} * * 2 / \mathrm{s})\end{array}$} \\
\cline { 2 - 5 } & Average & Maximum & Average & Maximum \\
\hline T1 & 2.30 & 4.88 & 0.25 & 16.03 \\
\hline T2 & 2.38 & 5.30 & 0.37 & 19.85 \\
\hline T3 & 2.53 & 5.41 & 0.44 & 23.64 \\
\hline T4 & 2.72 & 5.85 & 0.48 & 25.27 \\
\hline T5 & 2.31 & 5.29 & 0.44 & 26.01 \\
\hline T6 & 2.54 & 5.87 & 0.57 & 26.10 \\
\hline T7 & 2.66 & 6.88 & 0.69 & 25.81 \\
\hline T8 & 2.65 & 6.77 & 0.77 & 27.62 \\
\hline T9 & 2.51 & 6.73 & 0.69 & 27.80 \\
\hline T10 & 2.49 & 6.07 & 0.78 & 37.13 \\
\hline
\end{tabular}

The maximum overtopping rates from $\mathrm{B} 2 \mathrm{D} / \mathrm{B} 1 \mathrm{D}$ are associated with the individual waves in the sea state simulated. The CMS and Eurotop manual in Chapter 3 used values of the crest elevation $R_{c}=2,1.3$, and $1.6 \mathrm{~m}$ for Storm Conditions 1, 2, and 3, respectively, and assigned a value of 1 to various empirical parameters. The calculated overtopping rates from the 
Eurotop formula for Storm Conditions 1, 2, and 3 were 1.56, 3.25, and 2.12 $\mathrm{m}^{2} / \mathrm{sec}$, respectively. If $\mathrm{B} 2 \mathrm{D} / \mathrm{B} 1 \mathrm{D}$ based values of $\mathrm{R}_{\mathrm{c}}, \mathrm{H}_{\mathrm{s}}$, and mean water level were used in the Eurotop empirical formula for Storm Conditions 1, 2, and 3 , the overtopping rates would be $1.12,2.00$, and $1.66 \mathrm{~m}^{2} / \mathrm{sec}$, respectively, corresponding to approximately a $30 \%$ reduction. The mean average overtopping rates (e.g., mean value for transects T6 to T10) for the three storms from B2D/B1 are $0.62,0.99$, and $0.70 \mathrm{~m}^{2} / \mathrm{sec}$, respectively. These mean values for $\mathrm{T} 6$ to T10 indicate that the average overtopping rate estimates from B2D/B1D are approximately 50\% of the Eurotop estimates obtained using the local wave parameters and the real crest freeboard of the individual transect profiles and are discussed next.

If the design crest freeboard was used instead of the real crest height of individual transects, the overtopping rate estimates for the three storms would be $1.46,2.57$, and $2.14 \mathrm{~m}^{2} / \mathrm{sec}$, respectively, which are comparable to the Eurotop estimates reported in Chapter 3. However, it is emphasized that Chapter 3 estimates were based on a $4.2 \mathrm{~m}$ average design crest freeboard of the dike along the Legs 4 and 5. Because a finite number of transects (e.g., transects T6 to T10) covered the span of Legs 4 and 5, the estimates at some potentially damaged locations with lower crest heights could be greater than the estimates obtained with the average design crest freeboard.

Conversely, the estimates would be less at locations where the structure's crest was higher than the average crest design height. The overtopping rate estimates by the Eurotop empirical formula depend on the specified values of $\mathrm{R}_{\mathrm{c}}$ and $\mathrm{H}_{\mathrm{s}}$ and water level for a storm condition. The estimates from $\mathrm{B} 2 \mathrm{D} / \mathrm{B} 1 \mathrm{D}$ are based on geometry of the dike as represented in the profiles of individual transects, as well as model calculated local wave parameters and water levels along transect profiles.

\subsection{Future Fill 2025 scenario}

The CDF4 interior condition in year 2025 was the first future scenario that was modeled. Figure 4-9 shows the corresponding geometry. Figure 4-68 is a zoomed three-dimensional (3D) image of a small area of water near the $\mathrm{V}$-section. This small water area is represented with a light-blue strip at the corner of the $\mathrm{CDF}$, and the rest of interior was land filled. As an example, results of a test run for Storm Condition $1(\mathrm{Hs}=3.5 \mathrm{~m}, \mathrm{Tp}=10 \mathrm{sec}$, and WL $=2.3 \mathrm{~m}$ ) are shown in Figures 4-69 and 4-70. This choice was made because of partial or no successful runs for Storm Conditions 2 and 3 for the fill scenarios with larger waves and water levels. However, Storm Conditions 2 
and 3 results will be shown later for the raised-dike scenario. In this and previous simulations, the $2.3 \mathrm{~m}$ water level for this storm was applied to the entire modeling domain, including the interior of $\mathrm{CDF} 4$. Consequently, the submerged area inside $\mathrm{CDF}_{4}$ is larger. Furthermore, the existing dike structure with gaps and low elevation spots was used in this future fill scenario.

Figure 4-70 shows the change in wave height and mean water level along two interior arcs A and B for Storm Condition 1. Results for the existing $\mathrm{CDF}_{4}$ are provided for comparison. Wave height and water level decrease by approximately 20\% for Future Fill 2025 as compared to existing CDF4. The limited availability of water prevents growth of overtopping waves, and there is an increased dissipation of waves over the larger filled/land areas.

Figure 4-68. Future Fill 2025 scenario with a small area of water in the V-section.

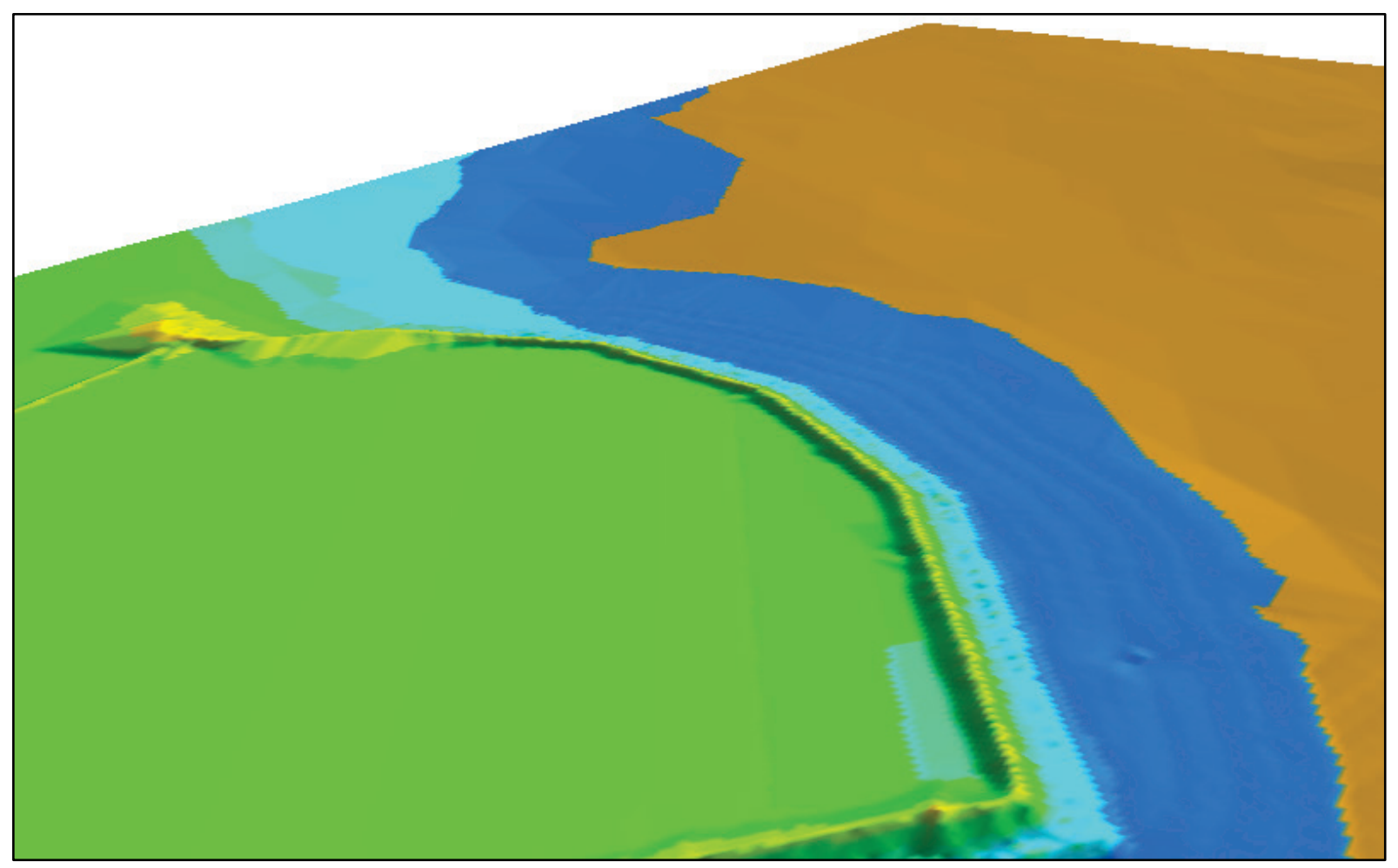


Figure 4-69. Storm Condition 1 results for Future Fill 2025; (a) wave surface elevation outside in the lake and (b) wave height field.
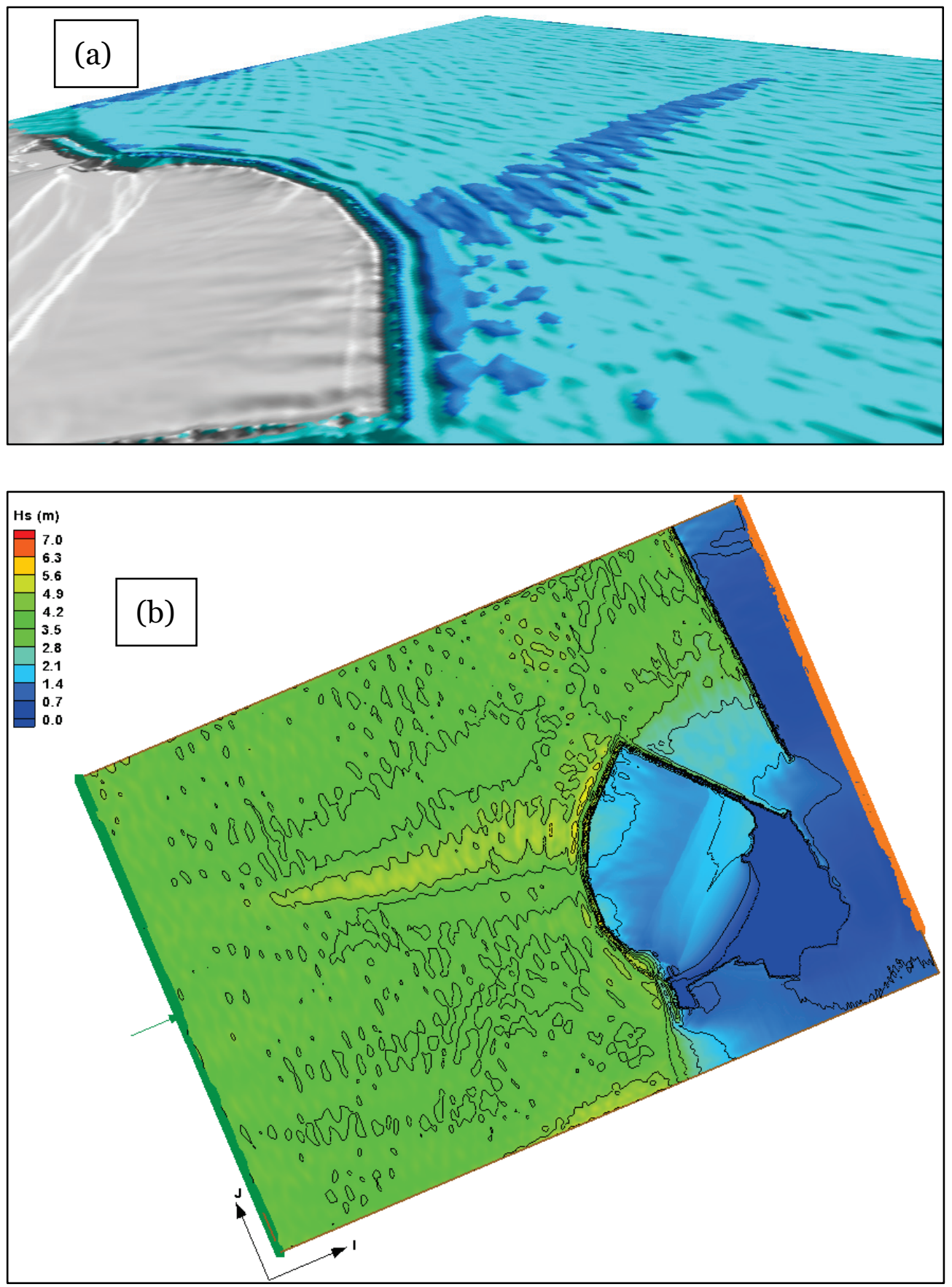
Figure 4-70. Wave height and water level variation along arcs $A$ and $B$ for Future Fill 2025 and existing CDF4, Storm Condition 1.

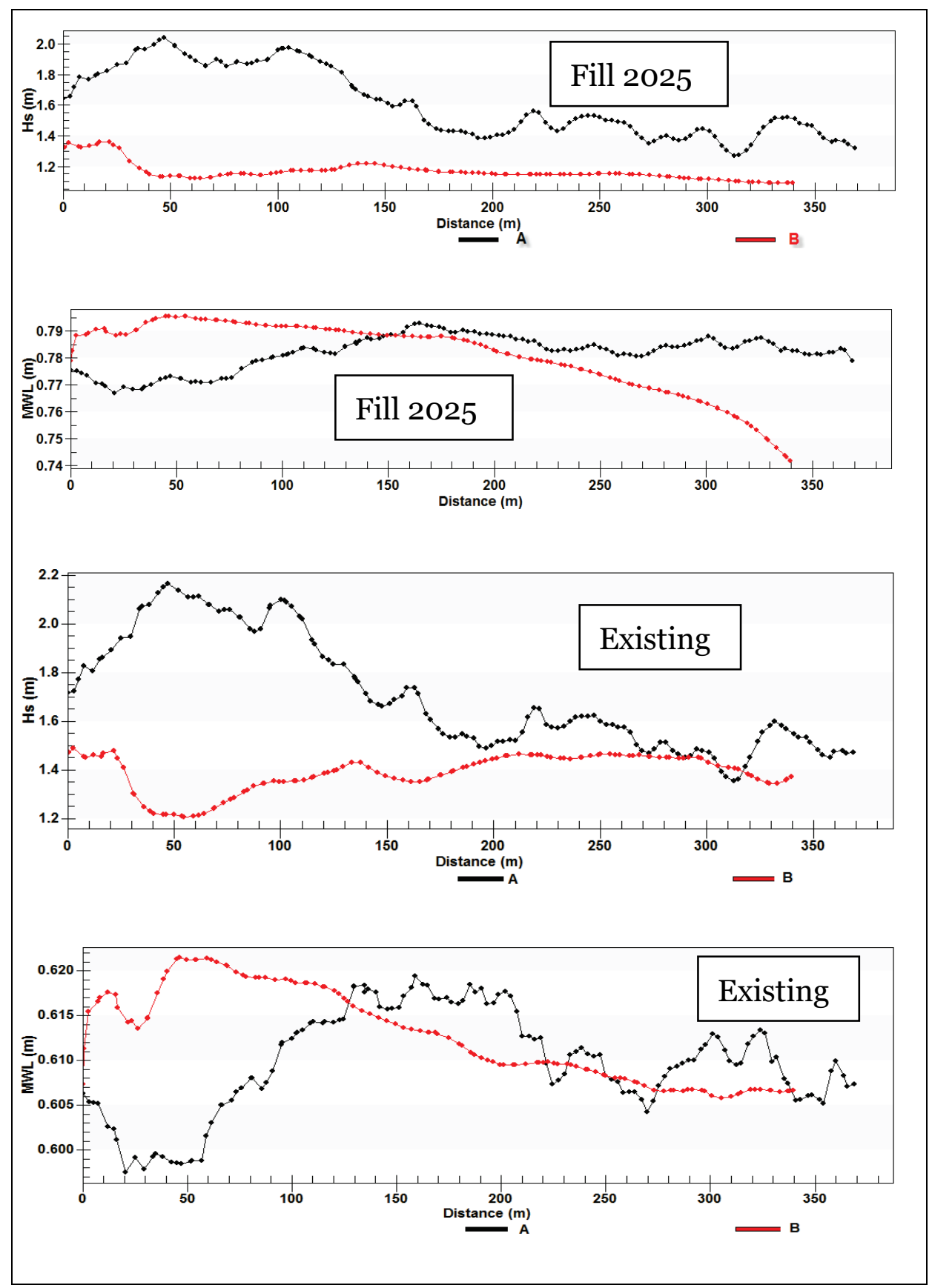

Figures 4-71 and 4-72 show snapshots of waves approaching the curved dike from the lake and waves entrapped inside Future Fill 2025. Because the interior of Future Fill 2025 was largely land (filled), images of the sea surface indicated overtopping waves advancing only a small distance over the land beyond the interior water area. Wave runup occurred over the land surface in these sections of $\mathrm{CDF}_{4}$ interior. In other sections distant from the water area, little or no movement of water surface occurred. In the animations, waves 
receded, rolling back into the water strip area in the interior of $\mathrm{CDF}_{4}$ adjacent to the Perimeter Dike. At times when wave breaking occurred, movement of the waves traveled farther up on the land (runup). There was no visible evidence of overtopping of the SEAB by waves trapped in the $\mathrm{CDF}_{4}$ interior for Storm Condition 1. Note that these observations apply only to Storm Condition 1 and cannot be extrapolated to Storm Conditions 2 or 3, the two more severe storms with only partial runs. This is the reason why results are provided here and in Appendix D for certain scenarios only for Storm Condition 1 but not for Storm Conditions 2 and 3.

Figure 4-71. Snapshot of wave surface elevation for Future Fill 2025 (Storm Condition 1).

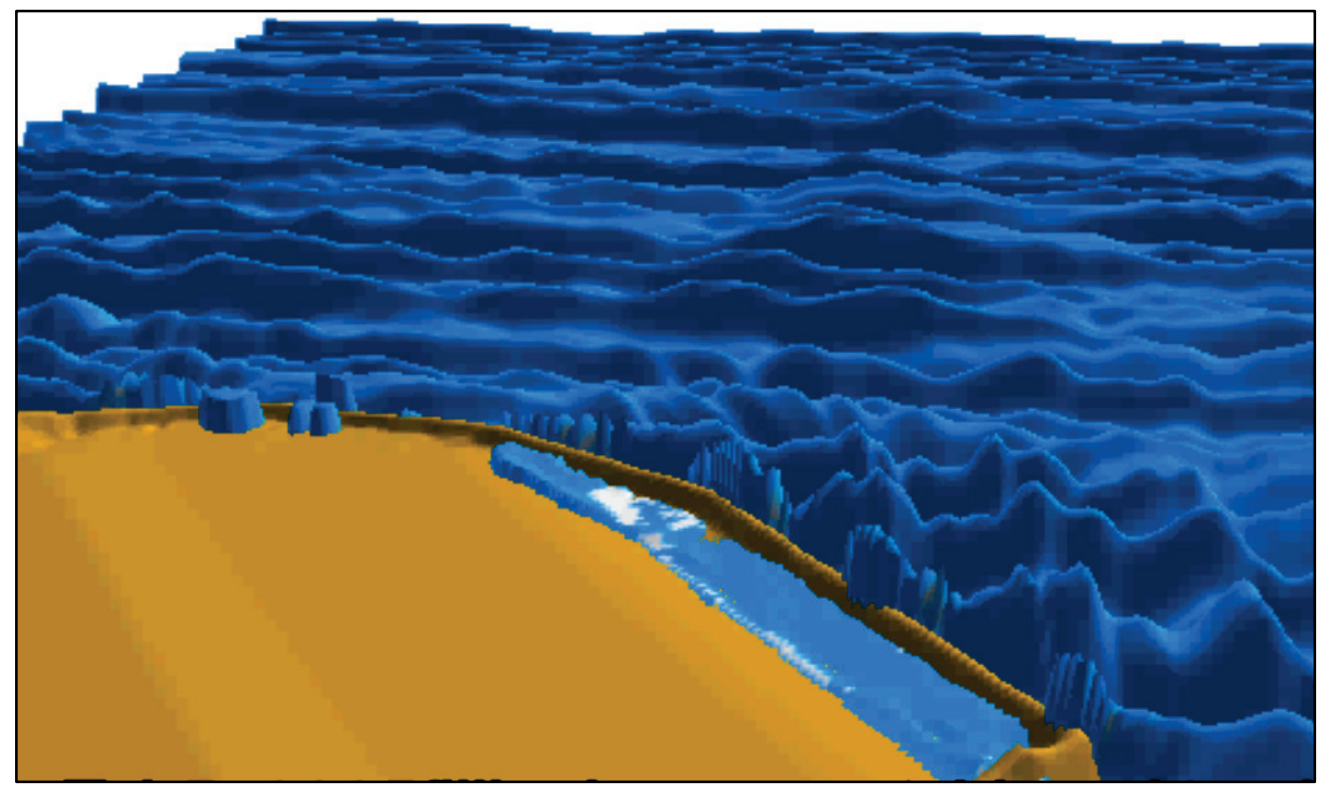

Figure 4-72. Snapshot of wave surface elevation for Future Fill 2025 (Storm Condition2).

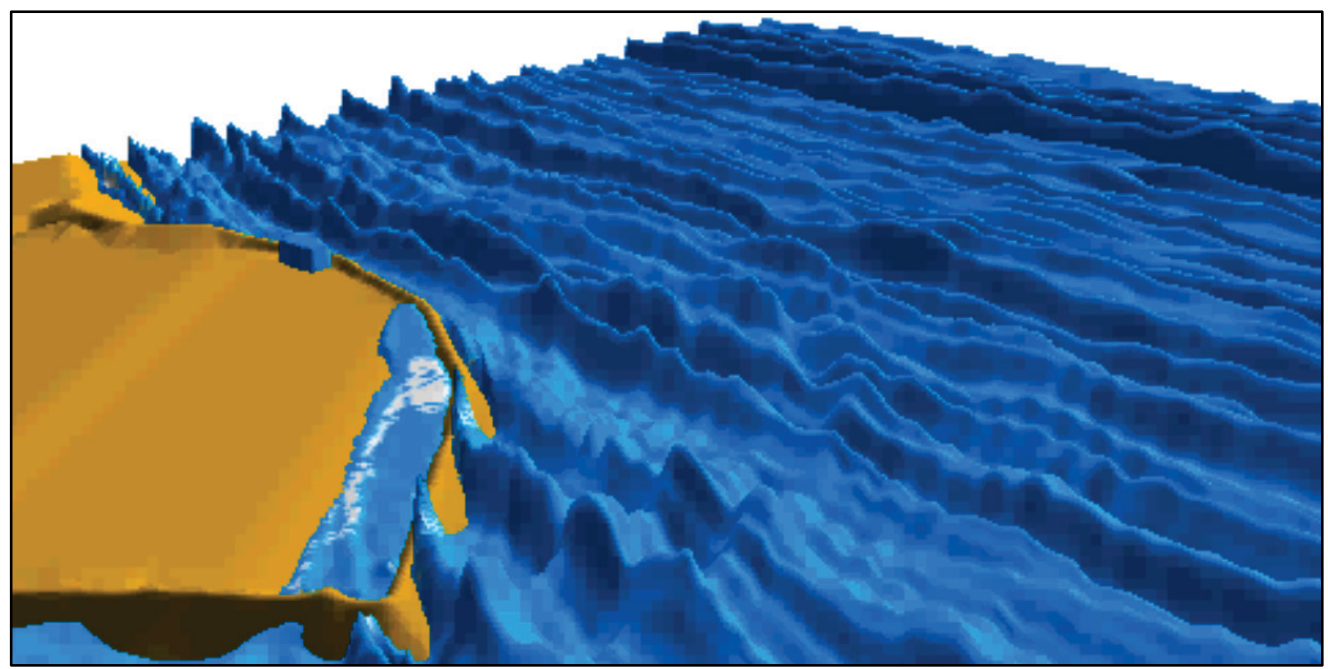




\subsection{Future Fill 2036 scenario}

Figure 4-10 shows the geometry for the future fill condition of $\mathrm{CDF}_{4}$ interior in year 2036. For this scenario the entire interior of $\mathrm{CDF}_{4}$ was land at a $+4 \mathrm{~m}$ fill elevation. Modeling attempts for this scenario were not successful due to the absence of any water inside the CDF4. Without water, it was not possible for overtopping waves to re-form and propagate over an entirely interior land surface inside CDF4. Simulations continued to the point after waves reached the land surface and water pockets started forming. Following a limited advance of the water pockets over the land surface, fluid movement became slow. When a continuous water strip developed covering the front section of the land surface, the model became unstable and the simulations stopped.

Instances of the fluid advancing over the land surface were captured from video images. These snapshots are depicted in Figures 4-73 through 4-79. The caption of each figure describes the status of fluid motion evolution over the interior land surface. A snapshot of the Storm Condition 1 simulation is shown in Figure 4-73. In this case, isolated water pockets developed on the top of the filled land surface.

Figure 4-73. Wave surface elevation snapshot for Future Fill 2036 (Storm Condition 1).

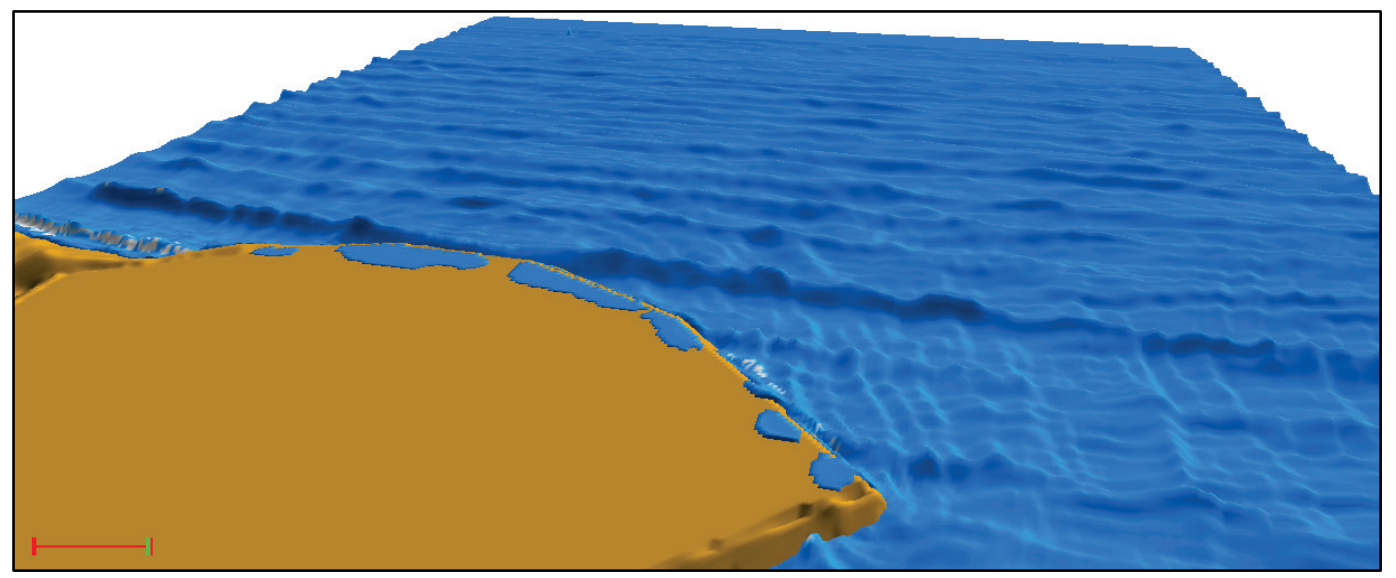

Storm Condition 2 produced more water pockets and continuous coverage of the front land section. These features are depicted by snapshots in Figures 4-74 through 4-79. It is surmised from these figures that the buildup of water inside $\mathrm{CDF}_{4}$ in the $\mathrm{V}$-section might be leading to a spillover of the SEAB. However, because the simulation failed, it was not possible to capture any spillover of the breakwater. Given the severity of water accumulation observed on the parts of interior filled land surface and if the simulation had continued further, it appeared that spillover was likely to occur. This could 
be prevented by raising the elevation of the first $100 \mathrm{~m}$ of the breakwater section (SEAB) or by lowering the filled land elevation level of the 2036 scenario.

Figure 4-74. Lakeside buildup of wave surface elevation for Future Fill 2036 (Storm Condition 2).

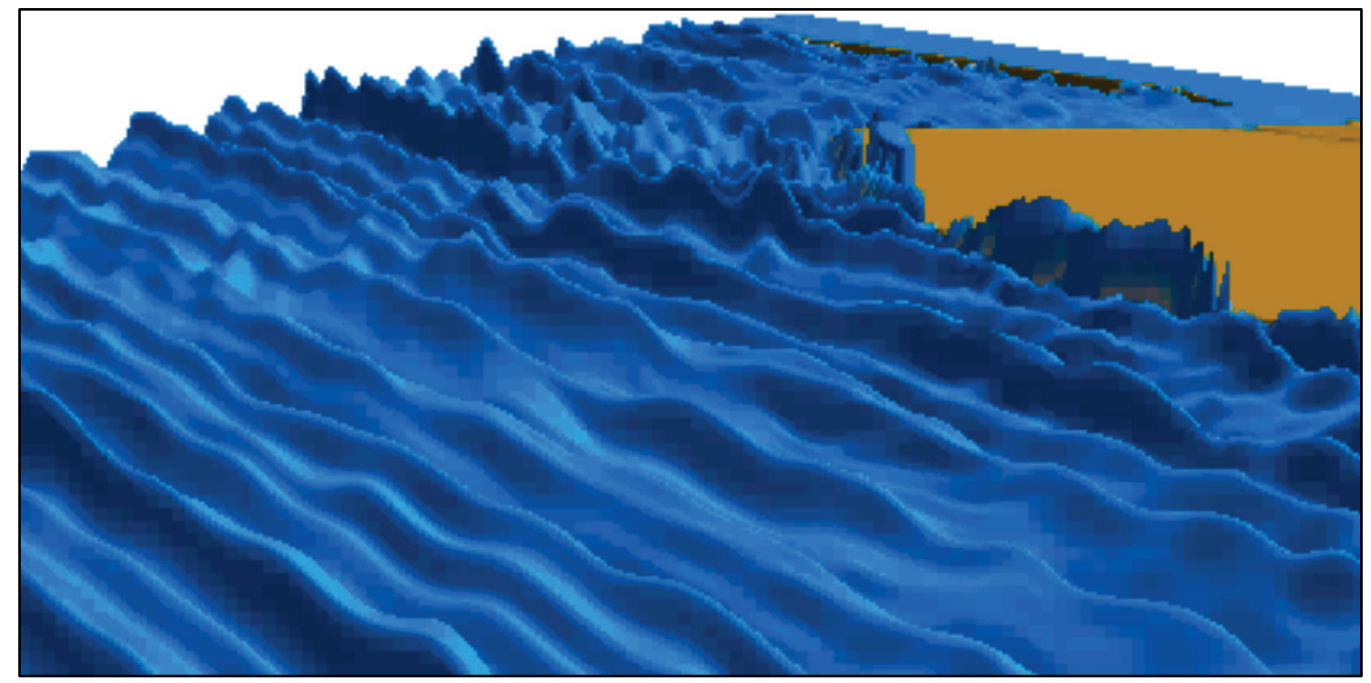

Figure 4-75. Overtopping waves reaching the top of interior land surface (Storm Condition 2).

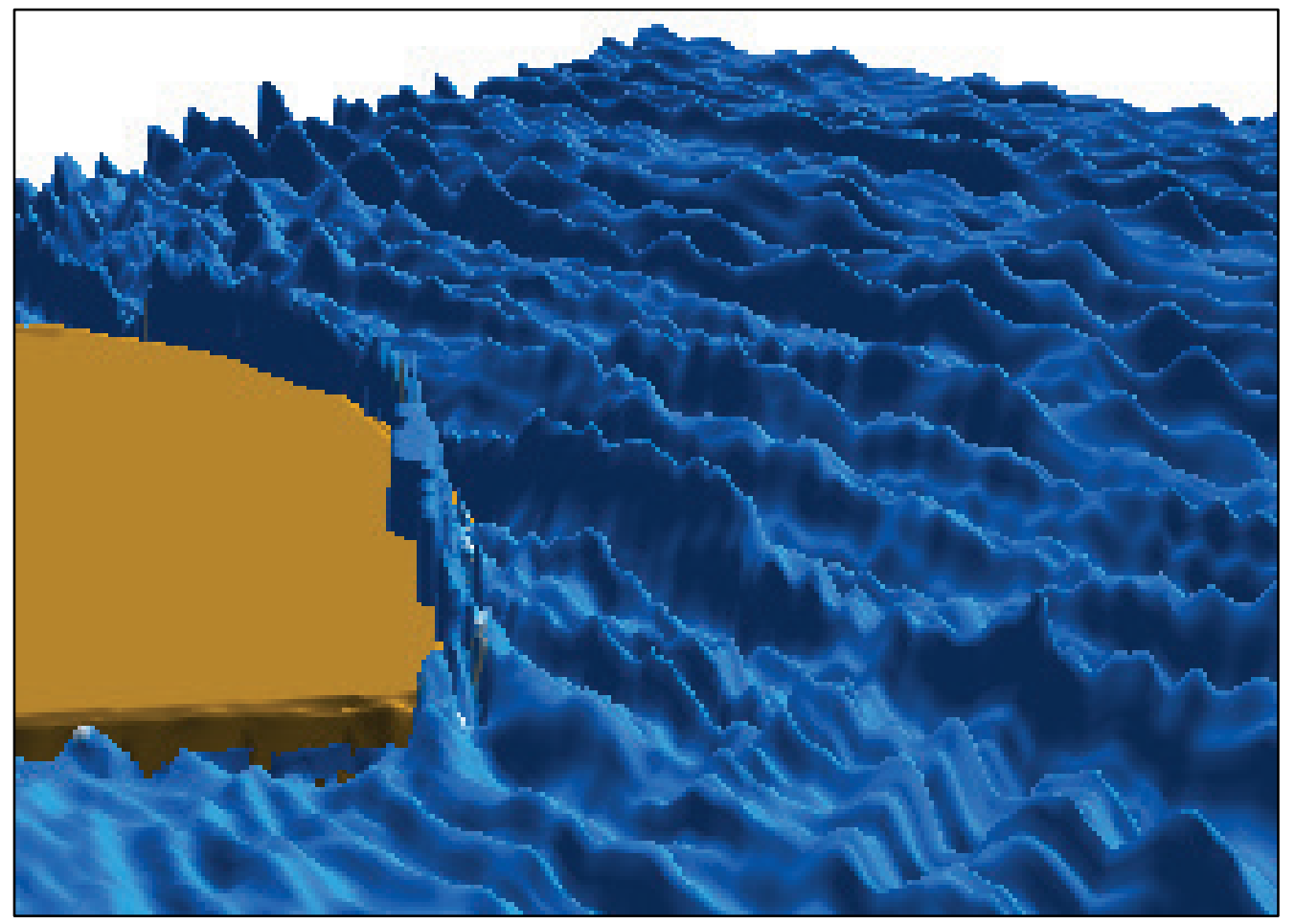


Figure 4-76. Water pockets developing at the top of interior land surface (Storm Condition 2).

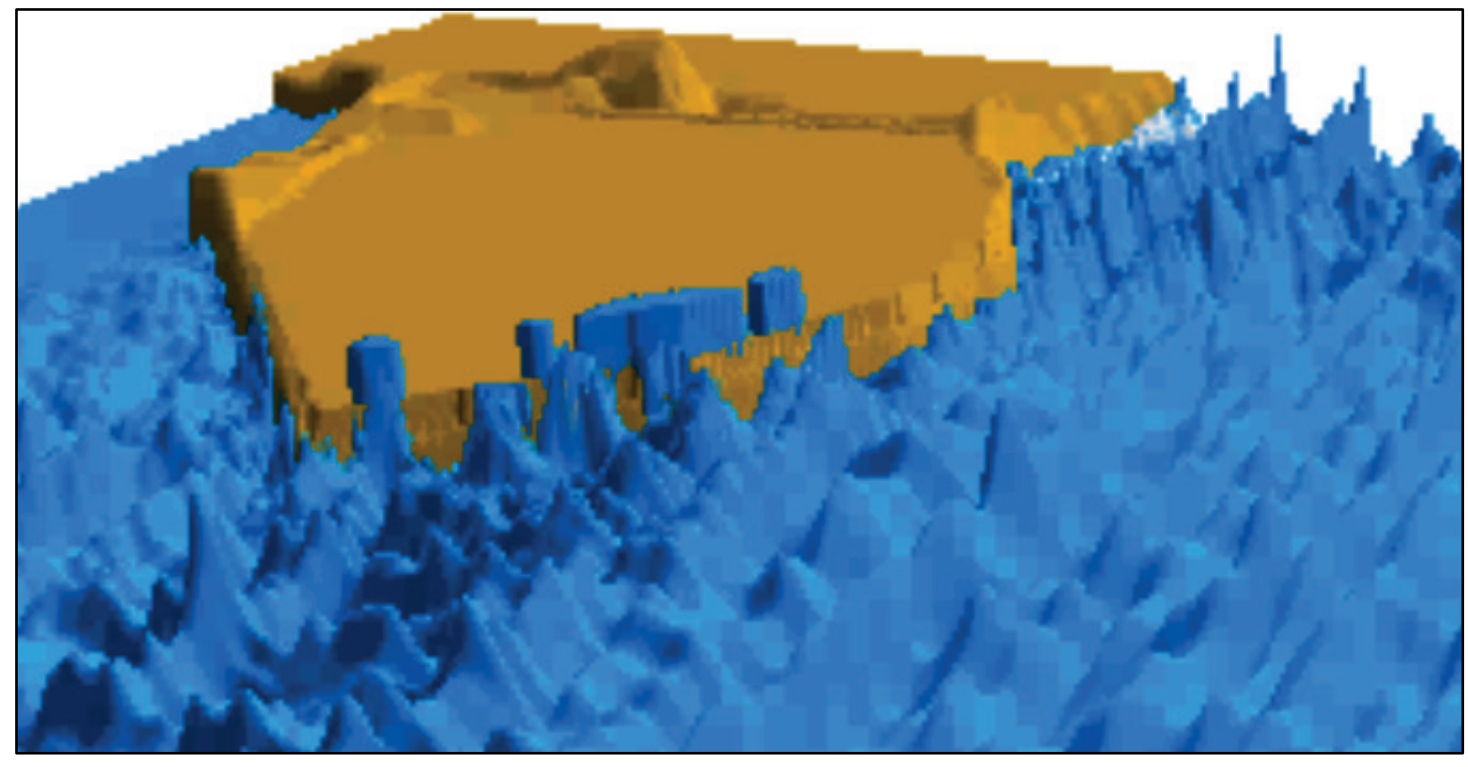

Figure 4-77. Water pockets advancing on top of interior land surface (Storm Condition 2).

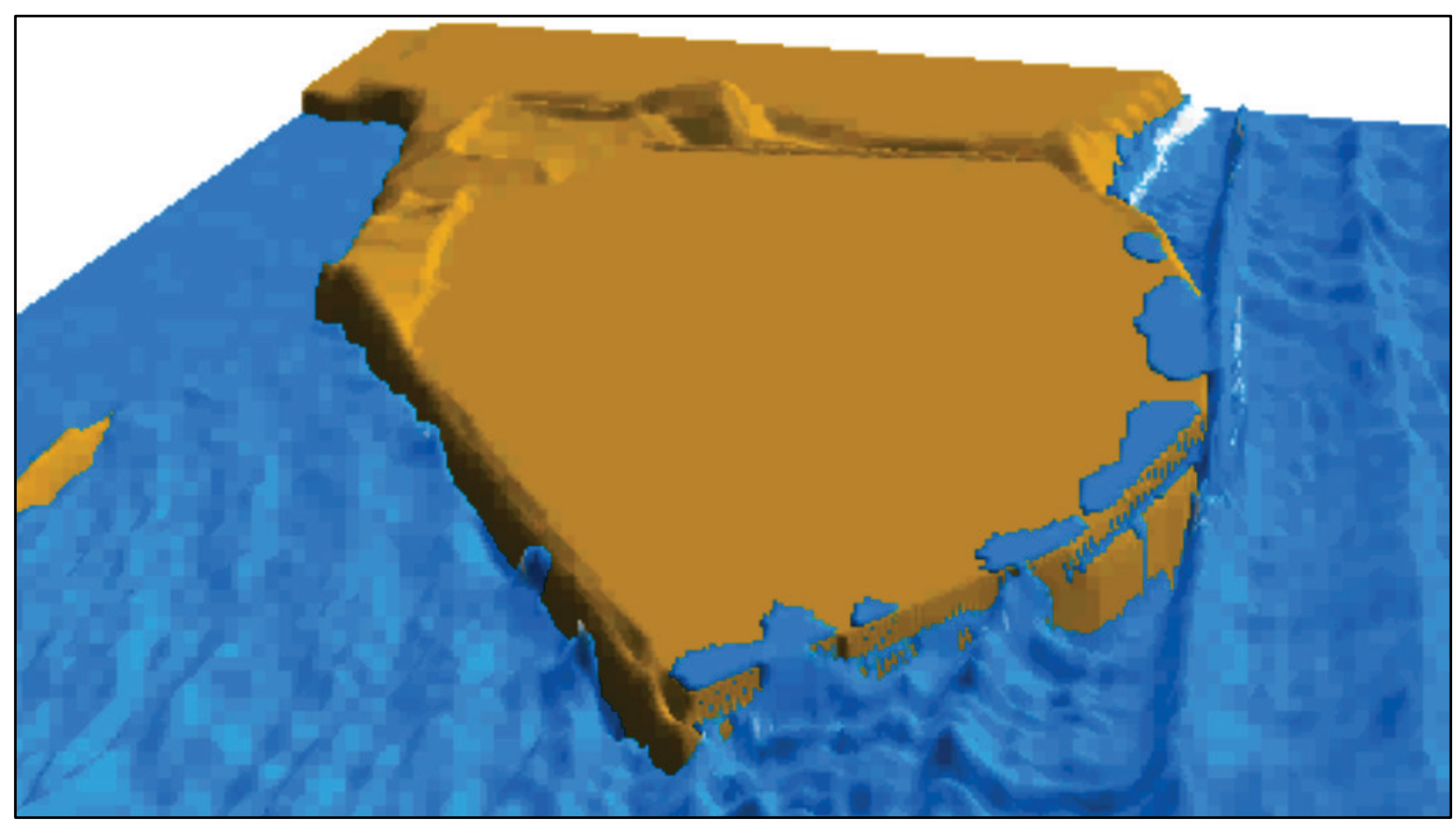


Figure 4-78. Water pockets increasing on top of interior land surface (Storm Condition 2).

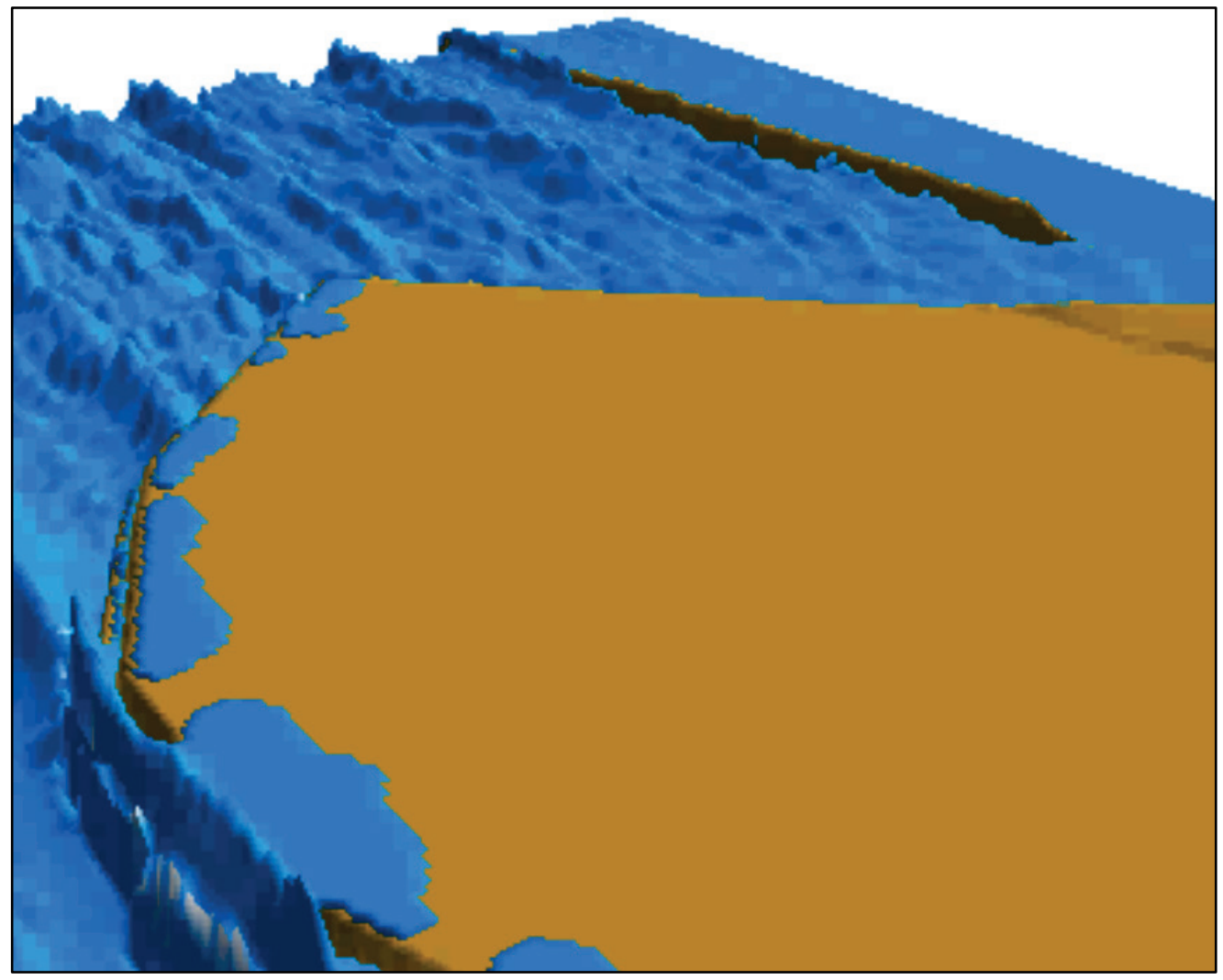

Figure 4-79. Continuous water coverage of front top section of interior land surface (Storm Condition 2).

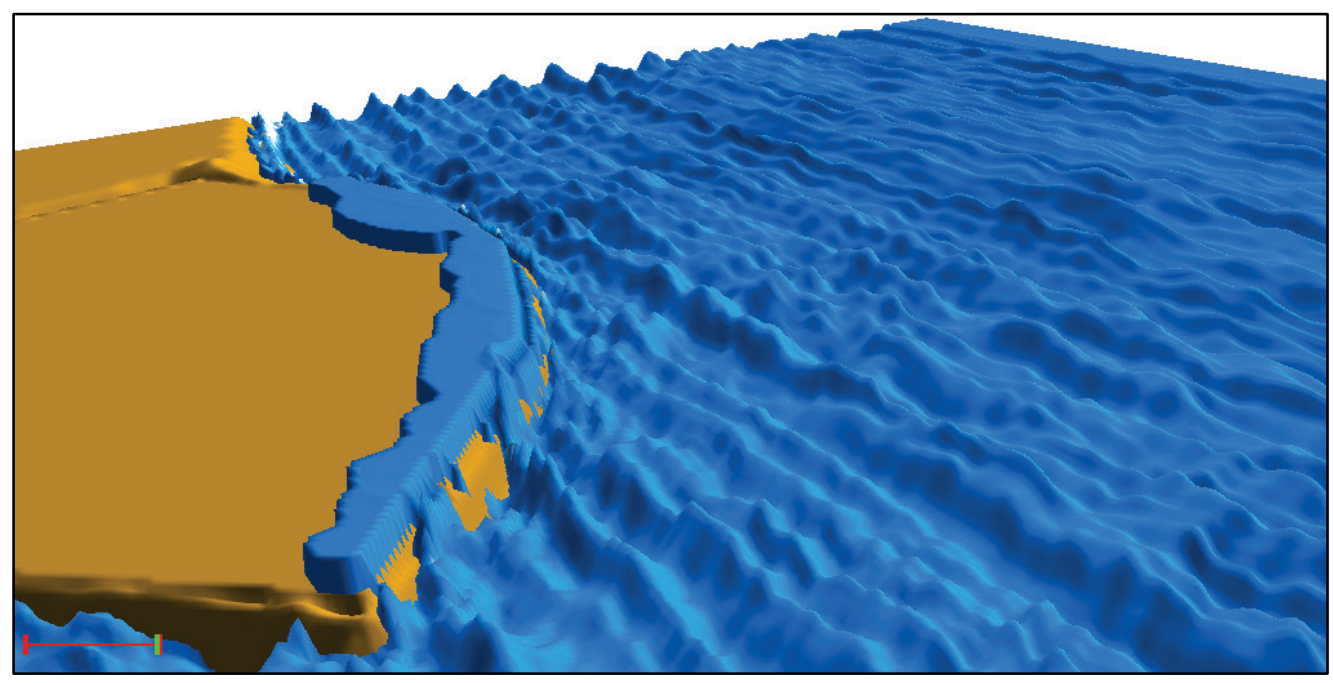




\subsection{Raised dike scenario}

Figures 4-11 and 4-12 show the geometry of the raised dike at elevation $+20 \mathrm{ft}(6.2 \mathrm{~m})$. Figure $4-80$ shows the elevated dike in B2D grid, where all spots with gaps and low elevation along the dike crest were filled to ensure a constant (6.2 $\mathrm{m}[20.3 \mathrm{ft}])$ crest elevation for the entire dike.

Figure 4-80. Image from B2D grid for the raised dike (gaps filled).

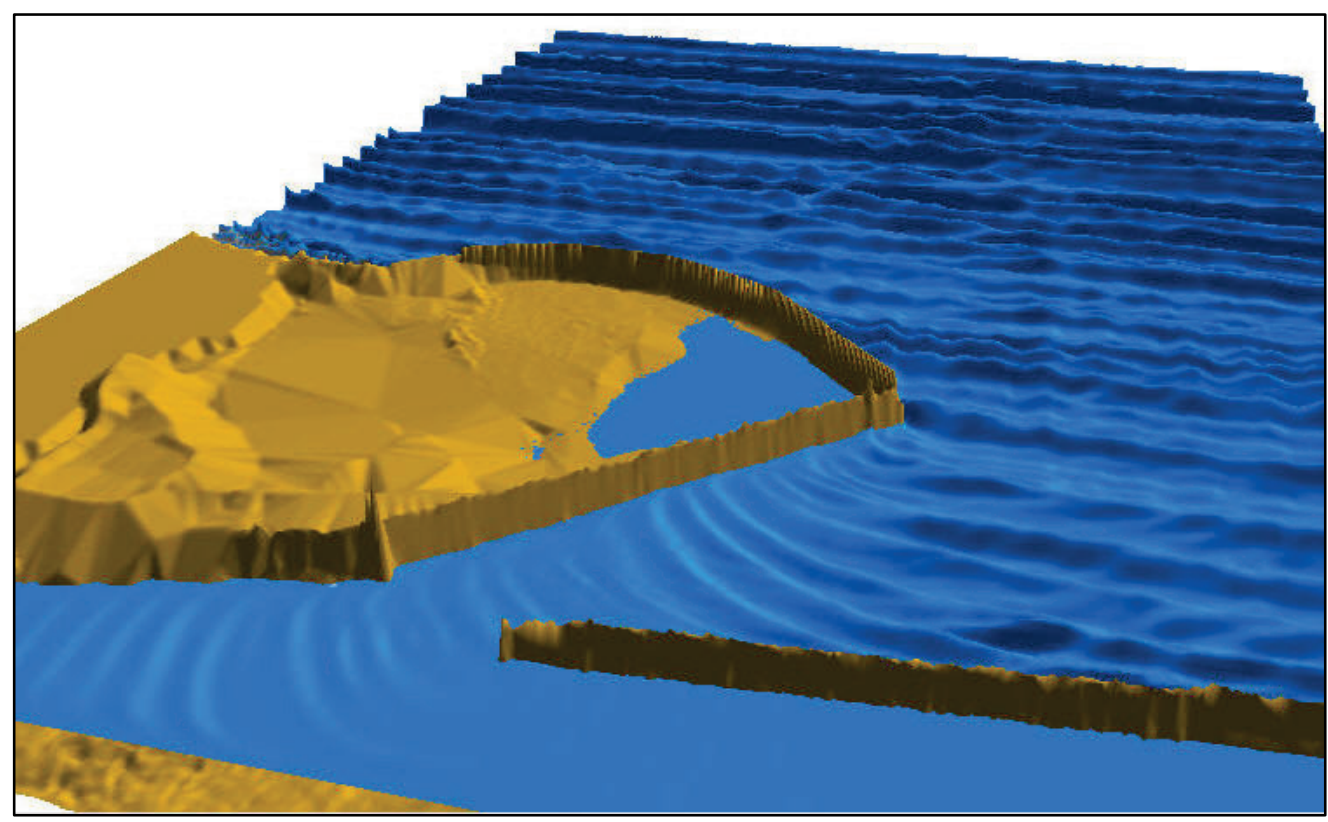

Figures 4-81 and 4-82 compare Storm Condition 3 results (with a WL=3 m) for the raised dike and existing dike, respectively. There are a number of spots along the existing dike crest that have gaps between the stones and core of structure. There are many spots along the existing dike with lower elevation. Irrespective of the storm considered, results presented in the previous sections indicated severe wave overtopping occurred at or near these spots with gaps and low elevation.

Comparison of these two results indicates that although overtopping continues to occur with the raised dike, there is a significant reduction in wave height and mean water level inside the $\mathrm{CDF}_{4}$ along arcs $\mathrm{A}$ and $\mathrm{B}$. The approximate maximum wave height and mean water level of $2.3 \mathrm{~m} \mathrm{(7.5} \mathrm{ft})$ and $0.8 \mathrm{~m}(2.6 \mathrm{ft})$ for the existing dike are reduced by the raised dike to 0.7 $\mathrm{m}(2.3 \mathrm{ft})$ and $0.39 \mathrm{~m}(1.3 \mathrm{ft})$, respectively. This benefit of the raised dike should be weighed against the additional cost of raising the entire dike to $20 \mathrm{ft}(6.2 \mathrm{~m})$ elevation. 
Figure 4-81. Hs and MWL with a raised dike (Storm Condition 3).

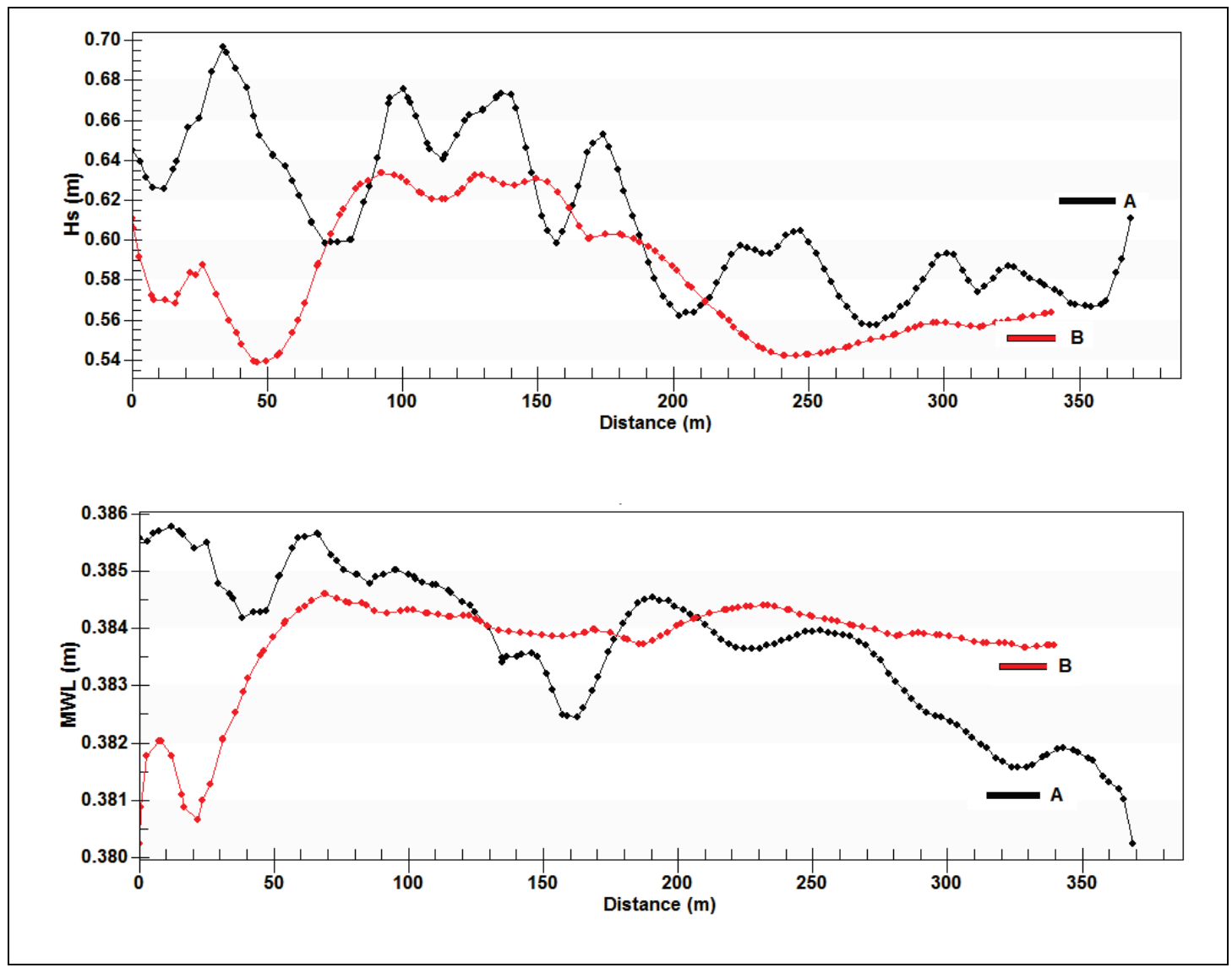

Figure 4-82. Hs and MWL for existing dike (Storm Condition 3).

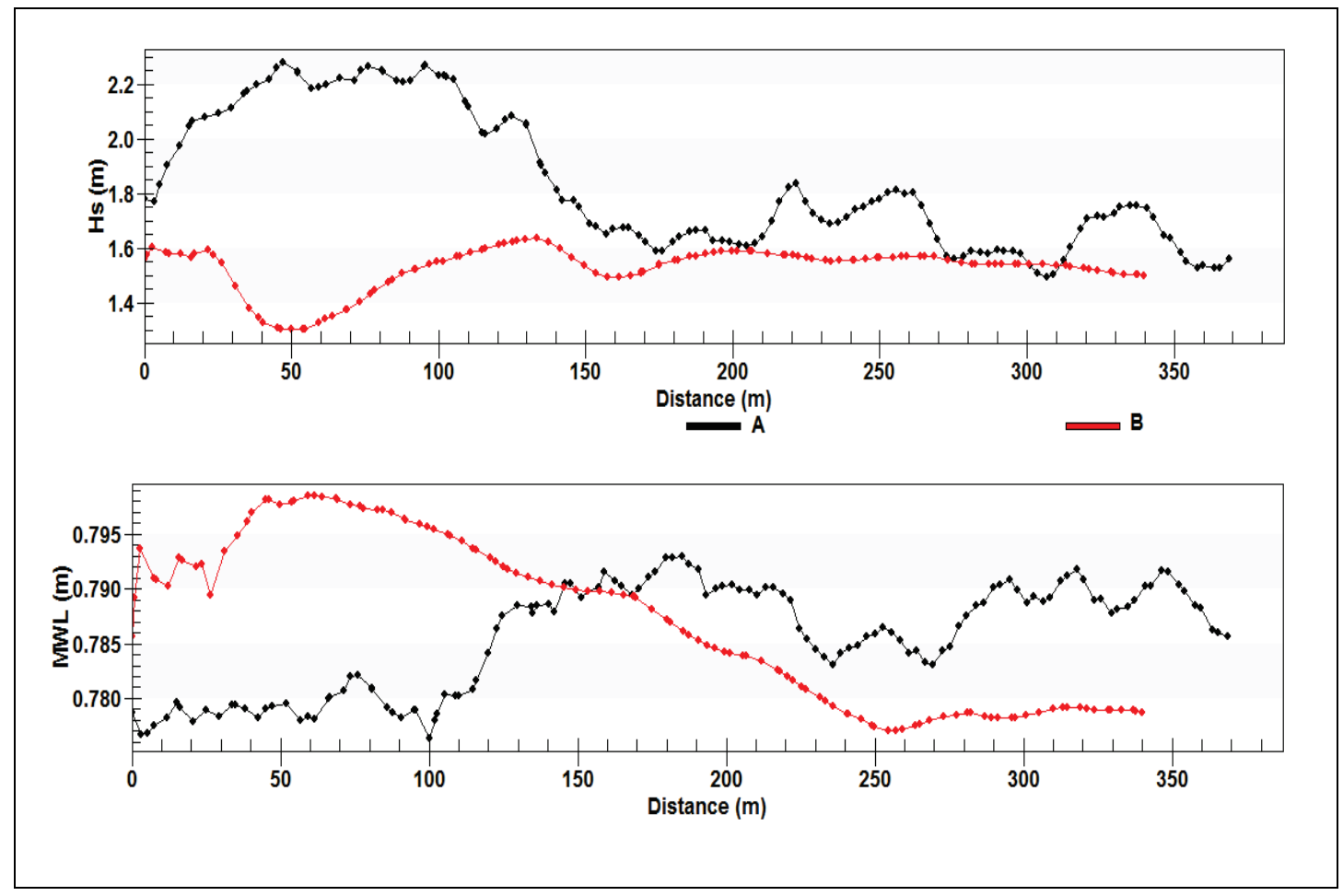


Figures 4-83 and 4-84 show Storm Condition 2 results for the raised dike and existing dike with $\mathrm{WL}=3 \mathrm{~m}$, respectively. These results for the raised and existing dike are similar to the Storm Condition 3. This is not surprising because only the incident wave height is different between these storms.

Figure 4-83. Hs and MWL with a raised dike (Storm Condition 2).

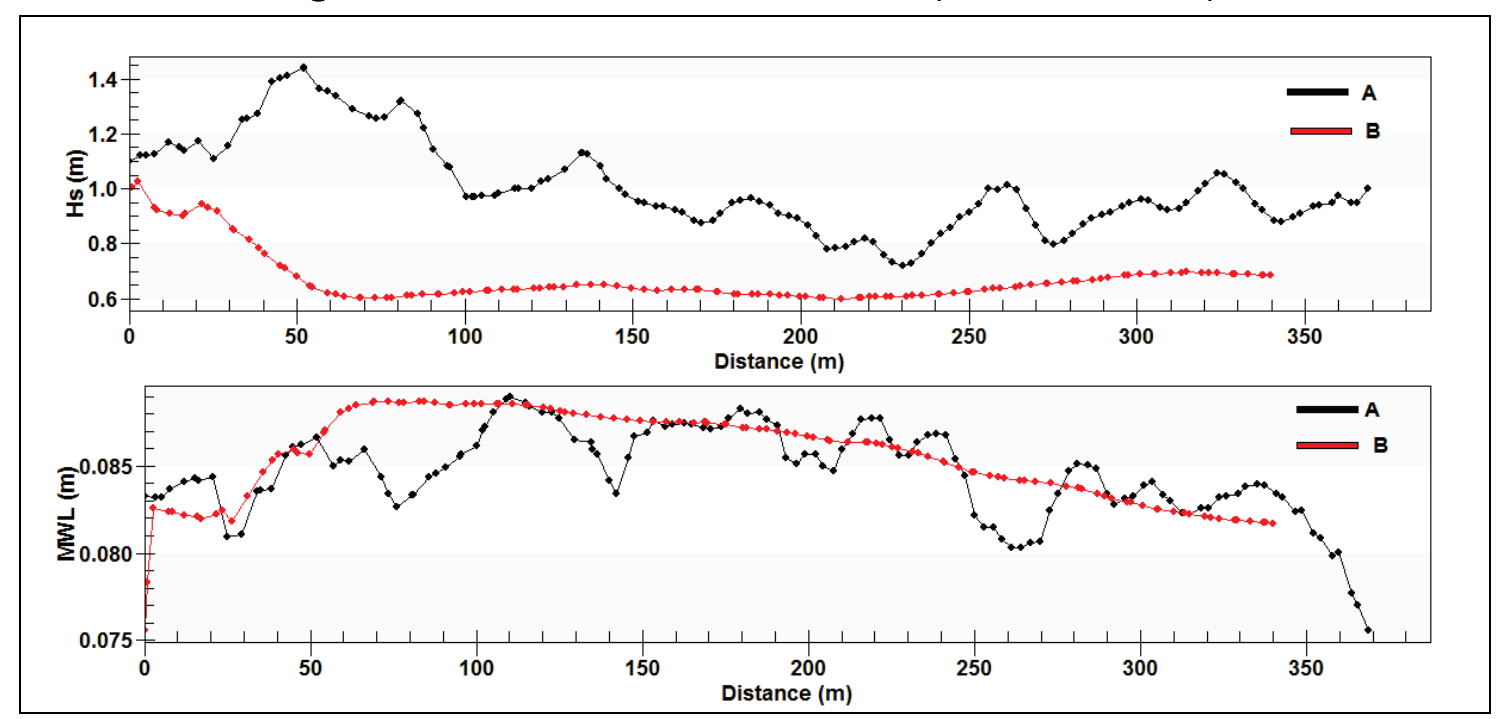

Figure 4-84. Hs and MWL for existing dike (Storm Condition 2).

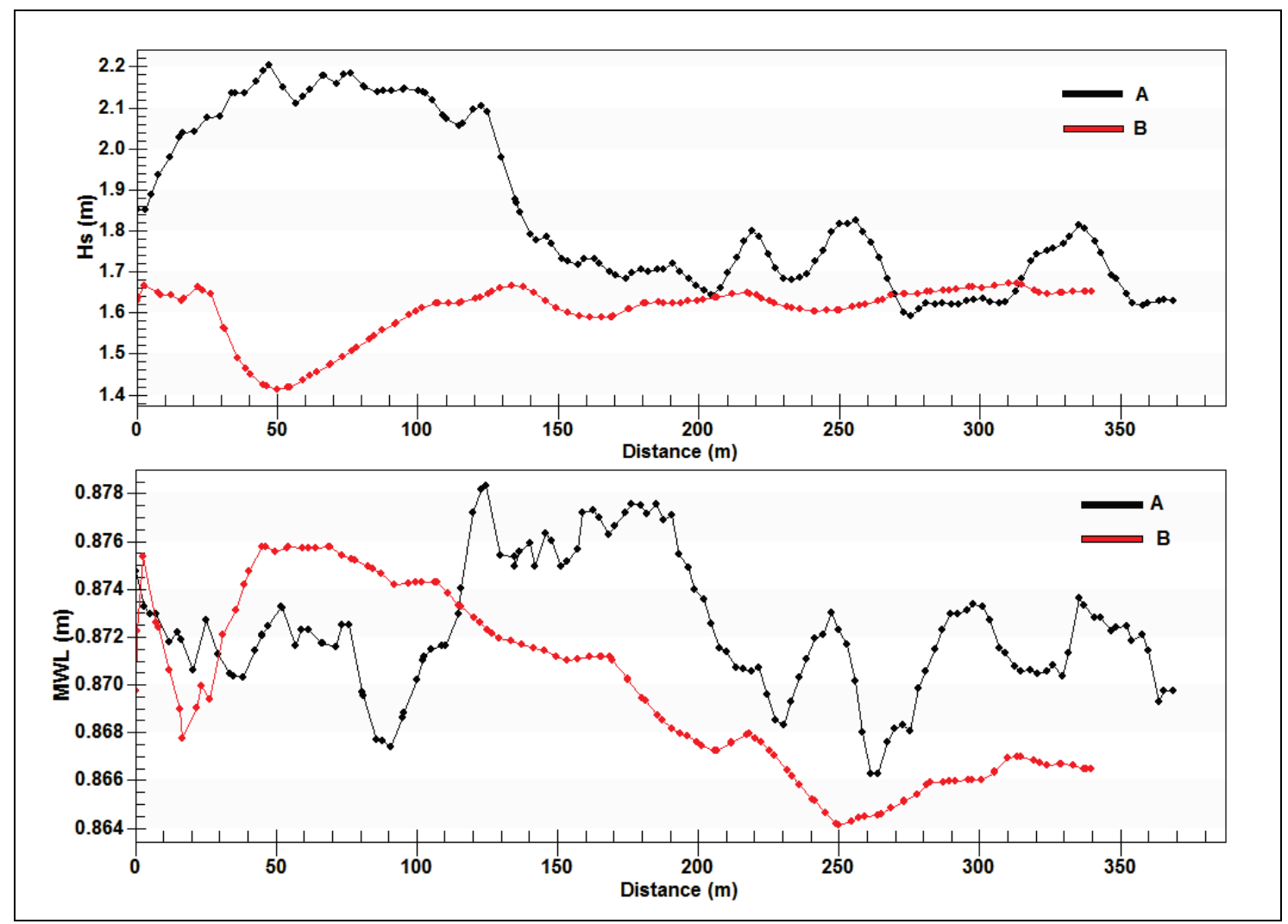


Comparison of existing and raised dike Storm Condition 2 results indicates that the raised dike did not eliminate overtopping while wave height and mean water level inside the $\mathrm{CDF}_{4}$ (along arcs $\mathrm{A}$ and $\mathrm{B}$ ) are reduced significantly. The maximum wave height for the existing dike is reduced from $2.2 \mathrm{~m}(7.2 \mathrm{ft})$ to $1.4 \mathrm{~m}(4.6 \mathrm{ft})$ by the raised dike. The mean water level is reduced from $0.9 \mathrm{~m}(3.0 \mathrm{ft})$ to $0.09 \mathrm{~m}(0.3 \mathrm{ft})$. A similar type of reduction was obtained for Storm Condition 3. 


\section{Summary and Conclusions}

The Buffalo Harbor CDF4 situated adjacent to the South Entrance Channel to Buffalo Harbor along the eastern shoreline of Lake Erie is a contaminated material holding facility that experiences periodic overtopping during significant storm events. There is concern that overtopping waves may cause the contaminated fine-grain sediments inside the CDF4 to mobilize, some of which may get transported over the (SEAB) into the south harbor entrance, navigation channel, and harbor complex. Concerns about wave overtopping at the $\mathrm{CDF} 4$ were investigated in this study by accounting for the effects of winds, waves, and water levels. Wave modeling estimates were developed for the existing $\mathrm{CDF}_{4}$ using the latest available bathymetric surveys and field data. Estimates of wave heights and overtopping rates were developed for the existing CDF4. Results from a preliminary model investigation for a raised dike and two future fill scenarios representing the condition of the CDF4 interior in Future Fill 2025 and Future Fill 2036 are also provided.

The present study focused on predictive estimates of wave heights and wave overtopping for the existing $\mathrm{CDF}_{4}$. Because flow and sediment transport modeling of the $\mathrm{CDF} 4$ interior were not considered, it was assumed that if flow occurred from the $\mathrm{CDF}_{4}$ over the breakwater, some sediment would exit as well. Wave processes in the exterior and interior areas of $\mathrm{CDF} 4$ were investigated to determine wave runup and overtopping for the existing CDF system using two different classes of wave models. These were CMS-Wave, a spectral wind-wave generation, growth and transformation model, and a nonlinear Boussinesq-type wave model (BOUSS-1D/2D).The large-domain steady-state spectral wave model CMS-Wave was coupled with the CMS-Flow to develop overtopping estimates, and also provided input conditions for detailed B2D wave modeling of the CDF4. B2D is a time-domain, nonlinear wave model designed specifically for wave-structure interactions and for modeling waves in ports and harbors. This small-domain nearshore wave model provided wave and overtopping estimates at the CDF4. It was also used to evaluate two future fill scenarios (Future Fill 2025 and Future Fill 2036) and a uniformly raised dike scenario to a crest elevation of $+20 \mathrm{ft}(6.2 \mathrm{~m})$. Details of CMS-Wave and B2D modeling are described in Chapter 3 and Chapter 4, respectively.

The focus of the modeling was to estimate wave overtopping of the curved dike, a structure designed to shelter the $\mathrm{CDF}_{4}$ interior from the metocean 
forcings in Lake Erie. Hydrodynamics of the CDF4 interior involving modeling of flow and sediment transport were not considered in this study. The coupled CMS-Wave and CMS-Flow produced estimates of storm-induced overtopping rate along the curved dike. It was necessary to include flow and water level in the CMS modeling to calculate the wave overtopping rate for $\mathrm{CDF}_{4}$, thus requiring the modeling. Finer resolution of the $\mathrm{CDF}_{4}$ and adjacent nearshore and channel areas was defined in regional (parent) and local (child) grids of the CMS models extending to parts of Buffalo Harbor and Lake Erie. Sediment and flow seepage were assumed to occur through the dike and SEAB by the design of these structures. Consequently, these structures were modeled three ways: (a) both as permeable, (b) only dike as permeable, and (c) both as impermeable, with a low permeability of wave and flow transmission through the structures.

CMS simulations were performed with the parent and child grids for three storm conditions; each storm simulation was conducted for permeable or impermeable structures and permeable dike. For Storm Conditions 1, 2, and 3 , hourly and total wave overtopping rates were calculated along the CDF4. From these storm simulations, CMS estimates of the wave overtopping rates were calculated. The estimates for the impermeable structure were greater than the permeable structure condition because of increased water levels and wave runup in front of and over the structure. For Storm Condition 1, the maximum overtopping rate for impermeable dike structure ranged from 22.6 to $33.4 \mathrm{ft}^{2} / \mathrm{sec}$ (2.1 to $3.1 \mathrm{~m}^{2} / \mathrm{sec}$ ). For a permeable dike, the rate varied from 3.6 to $6.0 \mathrm{ft}^{2} / \mathrm{sec}\left(0.33\right.$ to $\left.0.56 \mathrm{~m}^{2} / \mathrm{sec}\right)$, indicating a higher total overtopping volume with an impermeable dike.

Waves and water levels from the combined CMS simulations were used as input in the B2D simulations. The inputs were extracted at the B2D's open boundary in the lake at approximately a $10 \mathrm{~m}$ water depth. Because extreme storm conditions representing the combination of a 2-year wave and water level and a combination of the 20-year wave and 10-year return period water level were selected for modeling, severe and complicated wave-dike interaction was expected. It was necessary to use 1D Boussinesq (B1D) to properly resolve details of the dike structure in calculation of wave runup and overtopping of the curved dike. In this $\mathrm{B} 2 \mathrm{D}$ to $\mathrm{B} 1 \mathrm{D}$ coupled framework, the input conditions to B1D were obtained from B2D simulations near the toe of structure at a distance of approximately one wavelength into the lake. With this modeling approach, the curved dike was divided into five segments, and 
1D transects perpendicular to these segments were used to calculate wave runup and overtopping using a fine grid resolution.

Calculated wave heights by B2D for Storm Conditions 1, 2, and 3 lakeward of the existing CDF4 exhibited a similar pattern (Figures 4-50, 4-51, and 4-52). There was a noticeable wave focusing zone outside in the lake and along the front face of dike. The phenomenon was not observed in the CMS-Wave results because of the grid resolution and model physics. The $3.5 \mathrm{~m}, 4.0 \mathrm{~m}$, and $3.6 \mathrm{~m} \mathrm{(11.5} \mathrm{ft,} 13.1 \mathrm{ft}$, and $11.8 \mathrm{ft})$ incident wave heights at the model offshore boundary for three storms reached $5.5 \mathrm{~m}, 5.8$, and $5.1 \mathrm{~m}(18.0 \mathrm{ft}$, 19.0, $\mathrm{ft}$ and $16.7 \mathrm{ft}$ ) at the maximum wave focusing areas along Segments 3, 4, and 5 . This increase was caused by strong wave-dike interaction, including wave reflection and diffraction by the dike and water levels associated with each storm.

Calculated wave heights inside the $\mathrm{CDF}_{4}$ were generally less than half the incident wave height in the lake. As an example, the maximum wave height in the interior north $\mathrm{V}$-section of CDF4 for Storm Condition 2 was $2.3 \mathrm{~m}(7.5 \mathrm{ft})$ after overtopping occurred. Waves inside the $\mathrm{CDF} 4$ dissipated energy rapidly due to breaking and frictional dissipation as moved farther into the interior area of CDF4. Calculated wave heights overtopping the dike in the west interior (closest to the lake) were consistently greater than waves reaching the middle and east sections of the $\mathrm{CDF}_{4}$ interior. For the three storms simulated, there was no visible evidence of overtopping of the SEAB by waves inside the $\mathrm{CDF}_{4}$. Water levels fluctuated in the interior of $\mathrm{CDF}_{4}$ in an opposite trend to wave heights (e.g., lower water levels occurring in the west and increasing water levels in the east section of $\mathrm{CDF}_{4}$ interior). For Storm Condition 2, water level increased inside the $\mathrm{CDF} 4$ on the east side (along the SEAB), remaining under $0.9 \mathrm{~m}(3 \mathrm{ft})$. Because of significantly reduced wave height at this section of $\mathrm{CDF}_{4}$ interior, such an increase in water level was not high enough to cause waves to overtop the SEAB that has an average elevation of greater than $4 \mathrm{~m}(13.1 \mathrm{ft})$.

The coupled B2D/B1D modeling shed much more light into the spatial and temporal changes in the wave runup and overtopping rate along ten transects (Figure 4-8) covering the entire curved dike from south to north ends. Model results (Figures 4-56 through 4-64) showed details about the variation of wave runup/overtopping along transects in time and space. The hydrographs of overtopping for each storm included instantaneous overtopping by individual waves. These time series records of overtopping were analyzed to 
develop statistical estimates of engineering interest (e.g., minimum, average, maximum, standard deviation, and RMS values) at the grid points of each transect profile. The average overtopping rates for each storm along ten transects and wave overtopping rate statistics were developed (see Figures 4-65 through 4-67 and Tables 4-2, 4-3, and 4-4). The estimates for three storms indicated average overtopping rates ranging from 2.2 to 11.8 $\mathrm{ft}^{2} / \mathrm{sec}\left(0.2\right.$ to $1.1 \mathrm{~m}^{2} / \mathrm{sec}$ ) and maximum overtopping rates from 150 to 450 $\mathrm{ft}^{2} / \mathrm{sec}$ (14 to $42 \mathrm{~m}^{2} / \mathrm{sec}$ ). Although two different wave models (the B2D and CMS) and different calculation methods were used in this study for overtopping estimates, comparison of estimates indicated the average overtopping rates from $\mathrm{B} 2 \mathrm{D} / \mathrm{B} 1 \mathrm{D}$ were comparable to the peak storm rates by CMS for permeable structures. The CMS overtopping estimates for impermeable structures were greater than the average rates by $\mathrm{B} 2 \mathrm{D} / \mathrm{B} 1 \mathrm{D}$ combo. Note that the maximum rates from $\mathrm{B} 2 \mathrm{D} / \mathrm{B} 1 \mathrm{D}$ represent rates for the individual waves in each storm's sea state while the CMS rates correspond to the spectral representation (e.g., significant wave height) of the storms. The average overtopping rate is recommended as it is widely used in engineering practice. A detailed discussion is provided in Chapter 4 concerning wave overtopping rate estimates from the two wave models and Eurotop manual. The discussion addresses issues related to model calculated versus empirical formula based estimates and their interpretation in engineering works.

Comparison of model results for the Future Fill 2025 scenario to the existing dike indicated an approximately $20 \%$ reduction in wave height and water level inside the small water area remaining inside the CDF4. These simulations showed no visible evidence of wave overtopping of the SEAB by waves inside the CDF4 with the Future Fill 2025 scenario.

A successful simulation of the Future Fill 2036 scenario was not achieved. The observations made here are from partially completed simulations. In this scenario, the entire interior of $\mathrm{CDF}_{4}$ was land filled at $+4 \mathrm{~m}$ elevation. Simulations showed waves reaching the top of the land surface forming isolated water pockets, which expanded and grew in time as simulations progressed. The areas covered by thin films of fluid sheets (e.g., water pockets) were wetting and drying, and this led to numerical instabilities that stopped model runs. In some simulations, the water pockets accumulating on top of the land surface adjacent to the SEAB appeared to be close to overtopping the breakwater, but no eventual spillover of the structure could be captured because simulations terminated. 
For the raised dike at elevation $+20 \mathrm{ft}(6.2 \mathrm{~m})$, wave overtopping continued to occur, but there was a significant reduction in wave height and water level inside the CDF4. Compared to the existing dike, wave heights and water levels inside the CDF4 were reduced by factors of 2 to 3 .

In conclusion, based on results from two types of numerical modeling performed in this study, the repair and raising of the existing dike would have the greatest impact in reducing overtopping into the $\mathrm{CDF}_{4}$ interior. The repair should target parts of the existing dike with gaps and low elevation spots. Model results indicated raising the entire existing dike to a $+20 \mathrm{ft}(6.2 \mathrm{~m}$ ) elevation (e.g., $5 \mathrm{ft}(1.5 \mathrm{~m})$ additional increase of the design elevation) did not completely stop overtopping but reduced overtopping substantially. An additional $5 \mathrm{ft}(1.5 \mathrm{~m})$ increase of the dike crest height to a total elevation of $+25 \mathrm{ft}(7.6 \mathrm{~m})$ may prevent wave overtopping of the existing dike.

One finding of the present study was that for the three storm events, overtopping waves in the interior of $\mathrm{CDF}_{4}$ did not spill over (overtop) the SEAB. However, in some animations, waves did run up close to the top of breakwater where the crest of structure had low spots, and a spillover into the entrance channel appeared imminent. Because animations were using model calculations saved at a specific time interval (e.g., every $20 \mathrm{sec}$ ), the spillover of breakwater might have happened between animation snapshots. The severity of overtopping was clearly visible from animations. Combined with the types of waves that formed inside the CDF4, these suggest that SEAB spillover should not be discounted. Furthermore, wind-generated waves were not included in $\mathrm{B} 2 \mathrm{D} / \mathrm{B} 1 \mathrm{D}$. Therefore, the simulation underestimates the likelihood of SEAB overtopping. Wind forcing could increase water levels along the SEAB, and the wind setup might help to trigger overtopping at low spots along SEAB.

A second finding of the present study was that a significant amount of wave energy was present in the interior of the $\mathrm{CDF}_{4}$ and could certainly mobilize any fine sediment inside the existing CDF4. Hydrodynamics of interior and sediment transport were not in the scope of this study, which would require a 3D hydrodynamic modeling with wind, wave, water levels, currents, and sediment transport. Such a modeling study is necessary and highly recommended for addressing issues related to the mobility of sediments and fate and transport patterns inside the CDF4. For example, if depositional areas were to develop in parts of the interior and depending on 
characteristics of interior flow (circulation), these changes could lead easily to runup and overtopping of the SEAB. A $3 \mathrm{D}$ modeling study would require multiple sediment core samples and measured water levels at a few locations inside the $\mathrm{CDF}_{4}$.

The SEAB in the Future Fill 2025 and Future Fill 2036 conditions appeared to be vulnerable to spillover by flow and waves in the interior of CDF4. For either the partial or complete fill scenarios, there would be an increased propensity of interior sediments at the surface to get transported into the entrance channel or to the lake. Consequently, the capping of the top land surface would be necessary to prevent any loss of the CDF4 interior material exiting into adjacent water areas. One alternative to the capping is to increase the elevation of SEAB to manage the interior material of $\mathrm{CDF}_{4}$ into the channel, but this does not prevent contaminant material leaking to the lake unless the existing dike were also raised appropriately. 


\section{References}

Demirbilek, Z., L. Lin, O. G. Nwogu, K. K. Hathaway, W. C. Butler ,J. H. Podoski, and T. D. Smith. 2016. Assessment of Modifications for Improving Navigation at Hilo Harbor, Hawaii. ERDC/CHL TR-16-9. Vicksburg, MS: U.S. Army Engineer Research and Development Center.

Demirbilek, Z., L. Lin, O. G. Nwogu, W. C. Butler, K. K. Hathaway, and T. D. Smith. 2015a. Kikiaola Light Draft Harbor Monitoring Plan Part 2: Numerical Wave Modeling for Evaluation of Structural Alternatives. ERDC/CHL TR-14-9. Vicksburg, MS: U.S. Army Engineer Research and Development Center.

Demirbilek, Z., L. Lin, O. G. Nwogu, J. A. Goo, and T. D. Smith. 2015b. Infrastructure Modification for Improving Navigation at Faleasao Harbor in American Samoa. ERDC/CHL TR-15-15. Vicksburg, MS: U.S. Army Engineer Research and Development Center.

Demirbilek, Z., and C. L. Vincent. 2015. "Water Wave Mechanics." Chapter 1. EM 1110-21100, Part 2. Coastal Engineering Manual. Vicksburg, MS: U.S. Army Engineer Research and Development Center.

Demirbilek, Z., and J. Rosati. 2011. Verification and Validation of the Coastal Modeling System, Report 1: Summary Report. ERDC/CHL TR-11-10. Vicksburg, MS: U.S. Army Engineer Research and Development Center.

Demirbilek, Z., O. G. Nwogu, D. L. Ward, and A. Sanchez. 2009. Wave Transformation over Reefs: Evaluation of One-Dimensional Numerical Models. ERDC/CHL TR09-1. Vicksburg, MS: U.S. Army Engineer Research and Development Center.

Demirbilek, Z., L. Lin, and O. G. Nwogu. 2008. Wave Modeling for Jetty Rehabilitation at the Mouth of the Columbia River, Washington/Oregon, USA. ERDC/CHL TR-o83. Vicksburg, MS: U.S. Army Engineer Research and Development Center.

Demirbilek, Z., and O. G. Nwogu. 2007. Boussinesq Modeling of Wave Propagation and Runup over Fringing Coral Reefs, Model Evaluation Report. ERDC/CHL TR-0712. Vicksburg, MS: U.S. Army Engineer Research and Development Center.

Demirbilek, Z., O. G. Nwogu, and A. K. Zundel. 2007a. Infra-Gravity Wave Input Toolbox (IGWT): User's guide. ERDC/CHL CHETN-I-73. Vicksburg, MS: U.S. Army Engineer Research and Development Center.

Demirbilek, Z., O. G. Nwogu, D. L. Ward. 2007b. Laboratory Study of Wind Effect on Runup over Fringing Reefs. Report 1: Data Report. ERDC/CHL TR 07-4. Vicksburg, MS: U.S. Army Engineer Research and Development Center.

Demirbilek, Z., L. Lin, and A. Zundel. 2007c. WABED Model in the SMS: Part 2. Graphical Interface. ERDC/CHL CHETN-I-74. Vicksburg, MS: U.S. Army Engineer Research and Development Center.

Demirbilek, Z., A. Zundel, and O. Nwogu. 2005a. BOUSS-2D Wave Model in SMS: I. Graphical Interface. ERDC/CHL CHETN-I-69. Vicksburg, MS: U.S. Army Engineer Research and Development Center. 
Demirbilek, Z., A. Zundel, and O. Nwogu. 2005b. BOUSS-2D Wave Model in SMS: II. Tutorial with Examples. ERDC/CHL CHETN-I-70. Vicksburg, MS: U.S. Army Engineer Research and Development Center.

GLIN. 2017. Lake Erie Facts and Figures, the Great Lakes Information Network. http://www.great-lakes.net/lakes/ref/eriefact.html.

Irvine, K. N., G. P. Stein, and J. K. Singer. 1991. An Environmental Guide Book to the Buffalo River. Buffalo, NY: Center for Environmental Research and Education, SUNY College.

Lin, L., and Z. Demirbilek. 2005. "Evaluation of Two Numerical Wave Models with Inlet Physical Model." Journal of Waterway, Port, Coastal, and Ocean Engineering ASCE 131(4):149-161.

Lin, L., and Z. Demirbilek. 2012. Coupled BOUSS-2D and CMS-Wave Modeling Approach for Harbor Projects. ERDC/CHL CHETN-IV-84. Vicksburg, MS: U.S. Army Engineer Research and Development Center.

Lin, L., Z. Demirbilek, H. Mase, J. Zheng, and F. Yamada. 2008. CMS-Wave: A Nearshore Spectral Wave Processes Model for Coastal Inlets and Navigation Projects. ERDC/CHL TR-08-13. Vicksburg, MS: U.S. Army Engineer Research and Development Center.

Lin, L., Z. Demirbilek, and H. Mase. 2011a. "Recent Capabilities of CMS-Wave: A Coastal Wave Model for Inlets and Navigation Projects." In "Proceedings, Symposium to Honor Dr. Nicholas Kraus.” Journal of Coastal Research Special Issue 59:7-14.

Lin, L., Z. Demirbilek, R. Thomas, and J. Rosati. 2011b. Verification and Validation of the Coastal Modeling System, Report 2: CMS-Wave. ERDC/CHL TR-11-10. Vicksburg, MS: U.S. Army Engineer Research and Development Center.

Nwogu, O., and Z. Demirbilek. 2010. "Infragravity Wave Motions and Runup over Shallow Fringing Reefs." ASCE Journal of Waterway, Port, Coastal, and Ocean Engineering. (http://dx.doi.org/10.1061/(ASCE)WW.1943-5460.0000050)

Nwogu, O., and Z. Demirbilek. 2006. "Nonlinear Wave Interaction with Submerged and Surface-Piercing Porous Structures." In Proceedings, 3oth International Conference on Coastal Engineering, San Diego, CA, 287-299.

Nwogu, O., and Z. Demirbilek. 2008. "Nonlinear Wave Transformation and Runup over Fringing Coral Reefs.” In Proceedings, 31st International Conference on Coastal Engineering, Hamburg, Germany.

Nwogu, O. 2006. "Boussinesq Modeling of Landslide-Generated Waves and Tsunami Runup.” Edited by P.L.-F Liu, H. Yeh and. C. Synolakis. In Advanced Numerical Models For Simulating Tsunami Waves and Runup Advances in Coastal and Ocean Engineering 10:273-278.

Nwogu, O. G., and Z. Demirbilek. 2001. BOUSS-2D: A Boussinesq Wave Model for Coastal Regions and Harbors. ERDC/CHL TR-01-25. Vicksburg, MS: U.S. Army Engineer Research and Development Center. 
Nwogu, O. 1994. "Nonlinear Evolution of Directional Wave Spectra in Shallow Water." In Proceedings, 24th International Conference on Coastal Engineering, Kobe, Japan, Vol. 1, 467-481.

Nwogu, O. 1993. "Alternative Form of Boussinesq Equations for Nearshore Wave Propagation." Journal of Waterway, Port, Coastal and Ocean Engineering, ASCE 119(6):618-638.

Sanchez, A., W. Wu, T. M. Beck, H. Li, J. D. Rosati, Z. Demirbilek, and M. Brown. 2011a. Verification and Validation of the Coastal Modeling System, Report 4: Sediment Transport and Morphology Change. ERDC/CHL TR-11-10. Vicksburg, MS: U.S. Army Engineer Research and Development Center.

Sanchez, A., W. Wu, T. M. Beck, H. Li, J. Rosati III, R. Thomas, J. D. Rosati, Z. Demirbilek, M. Brown, and C. Reed. 2011b.Verification and Validation of the Coastal Modeling System, Report 3: Hydrodynamics. ERDC/CHL TR-1-10. Vicksburg, MS: U.S. Army Engineer Research and Development Center.

Skaros, D. 2015. "Buffalo River Remedial and Restoration Work." The Great Lakes Dredging Dispatch 1(2). http://greatlakesdredging.net/files/GLDT-News-20151116.pdf.

U.S. Army Corps of Engineers (USACE). 2016. Buffalo Harbor, New York, Dredged Material Management Plan, Preliminary Assessment (457522): Summary of Findings and Recommendations. Buffalo, NY: U.S. Army Corps of Engineers. 


\section{Appendix A: Description of CMS}

The CMS-Wave module of the CMS was used for the numerical modeling overtopping estimates of waves at $\mathrm{CDF}_{4}$ in Buffalo, NY. A brief description of the CMS is provided here for completeness.

As shown in Figure A-1, the CMS is an integrated suite of numerical models for waves, flows, and sediment transport and morphology change in coastal areas. This modeling system includes representation of relevant nearshore processes for practical applications of navigation channel performance and sediment management at coastal inlets and adjacent beaches. The development and enhancement of CMS capabilities continues to evolve as a research and engineering tool for desk-top computers. CMS uses the SMS interface for grid generation and model setup as well as plotting and post-processing. Additional information about CMS is available (Demirbilek and Rosati 2011; Demirbilek et al. 2007a,b,c; Lin and Demirbilek 2012, 2005; Lin et al. 2011a, 2011b, 2008).

Figure A-1. The CMS framework and its components.

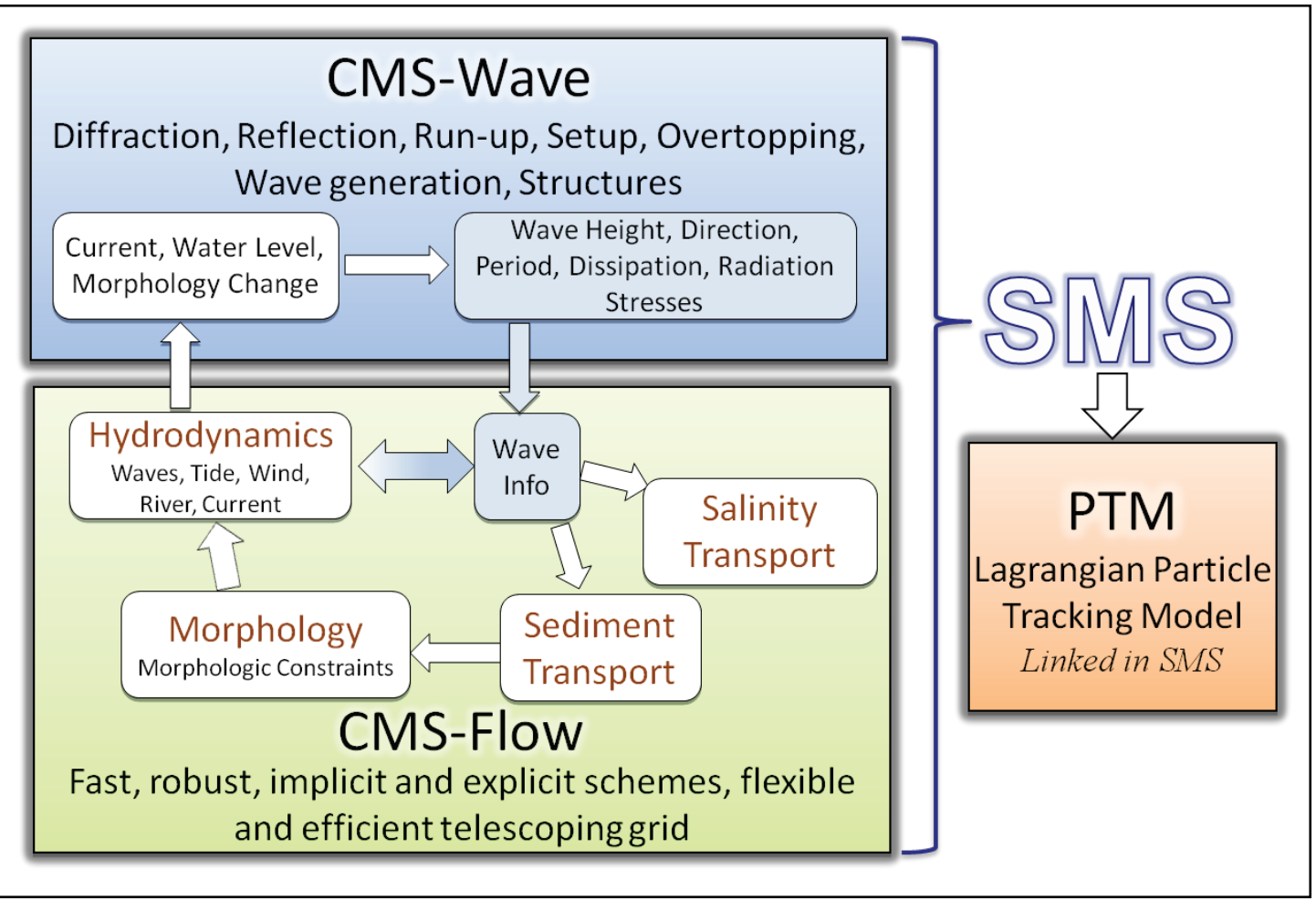


CMS uses the SMS interface for grid generation and model setup, as well as plotting and post-processing. The Verification and Validation (V\&V)

Report 1 (Demirbilek and Rosati 2011) and Report 2 (Lin et al. 2011) have detailed information about the CMS-Wave features and evaluation of model's performance skills in a variety of applications. Report 3 and Report 4 by Sanchez et al. (2011a and 2011b) describe coupling of wave-flow models and hydrodynamic and sediment transport and morphology change aspects of CMS-Flow. The performance of the CMS for a number of applications is summarized in Report 1, and details are described in the three companion V\&V Reports 2, 3, and 4.

The CMS-Wave, a spectral wave model, is used in this study given the large extent of modeling domain over which wave estimates were required. Details of the wind-wave modeling are described in Chapter 3 of this report. The main wave processes included in the CMS-Wave are wind-wave generation and growth, diffraction, reflection, dissipation due to bottom friction, white-capping and breaking, wave-current interaction, wave runup, wave setup, and wave transmission through structures. The height and direction of waves approaching the $\mathrm{CDF}_{4}$ and Buffalo Harbor entrance channel change due to wave shoaling, refraction, diffraction, reflection, and breaking. Waves propagating toward the CDF4 system and through the harbor entrance interact with bathymetry, surrounding land features and coastal structures. These features affect waves propagating and reaching the $\mathrm{CDF} 4$ protective structures, waves going over these structures and getting into the interior of $\mathrm{CDF}_{4}$, which can mobilize sediments inside the $\mathrm{CDF}_{4}$.

CMS-Wave model solves the steady-state wave-action balance equation on a non-uniform Cartesian grid to simulate steady-state spectral transformation of directional random waves at and around the $\mathrm{CDF}_{4}$ and Buffalo Harbor. CMS-Wave is designed to simulate wave processes with ambient currents in navigation channels, coastal inlets, and harbors. The model can be used either in half-plane or full-plane mode for spectral wave transformation (Lin et al. 2008; Demirbilek et al. 2007b). The half-plane mode is the default because in this mode CMS-Wave can run more efficiently as waves are transformed primarily from the lake ward boundary toward shore. See Lin et al. (2011, 2008) for features of the model and step-by-step instructions with examples for application of CMS-Wave to a variety of coastal inlets, ports, structures, and other navigation problems. Publications listed in the V\&V reports and this report provide additional information about CMS-Wave and its 
engineering applications. Additional information about CMS-Wave is available from the CIRP website: http://cirp.usace.army.mil/wiki/CMS-Wave.

Since the flow model was also used in this study, brief information is provided. The CMS-Flow is a $2 \mathrm{D}$, shallow-water wave model that can be used for hydrodynamic modeling (calculation of water level and current). Both the explicit and implicit versions of flow (circulation) model are available to provide estimates of water level and current given the tides, winds, and river flows as boundary conditions. CMS-Flow calculates hydrodynamic (depth-averaged circulation) sediment transport, morphology change, and salinity due to tides, winds, and waves.

The hydrodynamic model solves the conservative form of the shallow-water equations that includes terms for the Coriolis force, wind stress, wave stress, bottom stress, vegetation flow drag, bottom friction, wave roller, and turbulent diffusion. Governing equations are solved using the finite volume method on a non-uniform Cartesian grid. Finite-volume methods are a class of discretization schemes, and this formulation is implemented in finitedifference for solving the governing equations of coastal wave, flow, and sediment transport models. See the V\&V Reports 3 and 4 by Sanchez et al. (2011a and 2011b) for the preparation of the flow model at coastal inlet applications. Additional information about CMS-Flow is available from the CIRP website: http://cirp.usace.army.mil/wiki/CMS-Flow.

Although hydrodynamic, sediment transport, and morphology change modeling were not considered in this study, it is noted for future reference that there are three sediment transport models available in CMS-Flow: a sediment mass balance model, an equilibrium advection-diffusion model, and a non-equilibrium advection-diffusion model. Depth-averaged salinity transport is simulated with the standard advection-diffusion model and includes evaporation and precipitation. The V\&V Reports 1 through 4 describe the integrated wave-flow-sediment transport and morphology change aspects of CMS-Flow. The performance of CMS-Flow is described for a number of applications in the V\&V reports. 


\section{Appendix B: Description of BOUSS-2D}

The Boussinesq wave model BOUSS-2D (abbreviated as B2D) is an advanced modeling approach for nonlinear wave propagation nearshore (Nwogu and Demirbilek 2001). This technology was developed and implemented in the SMS in the 1990s through early 2000 and has since been used by Districts for navigation channels, inlets, harbors, coastal structures, moored vessels, floating breakwaters, and wave runup and overtopping on revetments, shorelines, and levees. Recent publications describe different applications of B2D model (Demirbilek et al. 2015, 2009, 2008, 2007a,b,c, 2005a,b; Nwogu and Demirbilek 2010, 2008, 2006, 2004; Nwogu 2009, 2007, 2006, 2000, 1996, 1994, 1993a,b). Additional information about B2D is available from these and other related publications in the References section of this report.

\section{B.1 Types of problems for B2D application}

The list below shows types of wave problems that can be simulated using Boussinesq wave models:

- harbor/port/marina problems: harbor resonance, harbor and marina infrastructure modifications

- generation of wave sub- and super-harmonics

- wave dissipation over porous media

- wave reflection and diffraction from structures, shorelines, and variable surfaces

- wave-wave interactions in shallow water

- channel deepening/widening/realignment

- wave-structure interactions: levees, flood walls, barriers, revetments, seawalls, groins, and breakwaters design and repair (coastal and inland)

○ wave runup/overtopping

- structure loading (wave forces)

- structure freeboard requirements

- frictional dissipation (i.e., waves on vegetated surfaces)

- wave interaction with an array of structure types

- embankment stability

- wave interaction with complex geometries of levees, navigation channels and canals, ports/harbors, etc.

- inundation mapping: overland propagation and runup

- bore propagation through rivers and canals 
- transient waves (tsunamis, sneaker waves)

- vessel-generated waves and ship wakes

○ vessel-generated waves and effect on shorelines

○ vessel-generated bed velocities and shear stresses

○ vessel interactions with other vessels and with locks and dams.

A few example applications are shown at the end of Appendix B.

\section{B.2 Background}

The B2D model was used for numerical modeling of wave runup/overtopping estimates at $\mathrm{CDF}_{4}$ in Buffalo, NY. The $\mathrm{CDF}_{4}$ study plan in Chapter 1 described the overall purpose of numerical modeling study and tasks while the implementation details of the wave modeling were provided in Chapters 3 and 4 of this report. Only a brief description of the $\mathrm{B} 2 \mathrm{D}$ features is provided here for completeness because details of model's theory, numerical computational schemes, and examples are available from the listed references.

Boussinesq models are essentially shallow-water models with extra dispersive and nonlinear terms. They excel under conditions of nonlinearity (large and/or long waves in shallow depths). Processes modeled well by Boussinesq models include nearshore wind-wave propagation, harbor resonance, nonlinear shoaling, runup and inundation, nearshore circulation, and tsunamis. Because Navier-Stokes models are not practical for field-scale problems, Boussinesq models presently are the computational tools of choice for calculating runup and overtopping of vertical or near-vertical walls or impulsive forces on structures. Boussinesq models can propagate vessel-generated waves if a source term is added for generation (i.e., moving pressure source or internal boundary). Boussinesq models are much better at this than shallow-water models because they include both short- and long-waves whereas shallow water wave equations (SWWEs) can only represent the long-wave component of the vessel-induced disturbances.

The B2D computes changes to waves caused by shoaling and refraction over variable bathymetry, reflection and diffraction from shorelines and structures, and nonlinear wave-current and wave-wave interactions. The internal Boussinesq equations defining the B2D do not contain adjustable parameters. Potential errors are introduced in numerical discretization of mathematical equations, imperfect boundary conditions, and physical 
processes that contain process-specific parameters, such as wave turbulence, dissipation, bottom friction, and boundary reflectivity. The B2D needs field data to calibrate the model parameters as it can simulate processes that cannot be estimated well in physical models (i.e., laboratory experiments) due to scaling effects. In the absence of field data, physical model data if available could be used in $\mathrm{B} 2 \mathrm{D}$ for validation and calibration of boundary conditions, material parameters, and numerical algorithms. Generally, errors in the nearshore wave estimates come from two sources: input to the model and the model itself, including errors in the incident wave conditions, bathymetry, and boundary specifications. The largest errors are associated with the specification of incident wave parameters and the simplification of wave breaking, dissipation processes, and contamination from model boundaries.

The B2D provides spatially and temporally varying wave, current, and water level parameter estimates for engineering problems. Estimates include significant wave height, peak period and direction, wave spectrum, time series of surface elevation, velocity and pressure, and wave-induced circulation. B2D model interface is operational in the SMS for grid generation and visualization of model results. The custom-built SMS interface of $\mathrm{B} 2 \mathrm{D}$ allows users to set up and run the model in an intuitive manner, with built-in safeguards (Demirbilek et al. 2005a,b). The B2D can be run on PCs, workstations, and super-computers.

The $\mathrm{B} 2 \mathrm{D}$ consists of a set of comprehensive numerical modeling systems based on a time-domain solution of Boussinesq-type equations for simulating waves (wind-waves and vessel-generated waves) and their propagation in coastal regions, harbors, and waterways. The B2D represents most of wave phenomena of interest in the nearshore zone for navigation projects, inlets, harbors, levees, structures, reefs, wetlands, ship wakes, wave-ship-bank interactions, and wave-current-structure interactions. The B2D-based engineering analysis systems may be used in navigation infrastructure design with a risk-based probabilistic design approach to evaluate life-cycle cost of alternatives, operation, and maintenance of coupled systems in deciding the benefit or negative consequences of structures in projects. The B2D has capability of replacing considerably more expensive physical models, with flexibility and generality for extension to sediment transport and morphology change, channel infilling, and water-quality issues. It is expected that the Corps O\&M budget for dredging navigation channels and expansion of ports/harbor economic capacity will continue to experience an increasing 
number of calls for deepening and widening channels and harbors to accommodate future fleets having larger, deeper draft, and faster vessels. Vessel-to-vessel and vessel-to-bank interactions and risk of accidents will also increase with these demands. Aging and natural deterioration of navigation structures increases vessel transit and maneuvering risks along the hightraffic shipping routes, channels, and ports.

Numerical models that solve Boussinesq-type water-wave evolution equations are commonly used to investigate surface wave propagation and transformation in coastal regions. Most of the models use finite difference schemes to discretize the equations over uniformly spaced rectangular grids (Nwogu and Demirbilek 2001). The popularity of finite difference schemes is largely based on their simplicity and ease of implementation. However, the use of structured grids can severely restrict the potential application of such models to complex boundary problems such as coastal flooding over complex topography, wave propagation in curved channels, wave interaction with coastal structures of arbitrary shape, and wave agitation in harbors of arbitrary shape. Because unstructured grids provide users the flexibility of modeling complex geometries and the grid resolution can be refined where needed such as near structures or in shallow regions, it was therefore highly desirable to develop an unstructured-grid version of the finite-difference B2D model used in civil and military works. The development of an unstructured-grid, finite-volume version of $\mathrm{B} 2 \mathrm{D}$ has been completed. This new model is being tested on super-computers, and its interface in SMS is under development.

The $\mathrm{B} 2 \mathrm{D}$ is designed to simulate wave processes with ambient currents at coastal inlets and in navigation channels. The model can be used for spectral wave transformation. See Lin and Demirbilek (2012) for step-by-step instructions for examples of coupled B2D and CMS-Wave modeling approach to harbor projects and other applications to coastal inlets, ports, structures, and other navigation problems. See Nwogu and Demirbilek (2001), Demirbilek et al. (2005a,b), and other publications listed in the References section for further information about the B2D and its engineering applications. Additional information about CMS-Wave is also available from these websites: http://cirp.usace.army.mil/wiki/Bouss-2D and http://www.xmswiki.com/xms/SMS:BOUSS-2D. 
In this study, the coupled $\mathrm{B} 1 \mathrm{D} / \mathrm{B} 2 \mathrm{D}$ models were used for nearshore wave modeling to investigate estimates of wave runup/overtopping of the $\mathrm{CDF} 4$ system. Details of B2D modeling are described in Chapter 4 of this report.

\section{B.3 Example applications}

The images in Figures B-1 through B-10 show some recent examples of B2D model applications. See References for other types of applications.

Figure B-1. BOUSS-2D calculated wave-induced current field for Pillar Point Harbor, California.

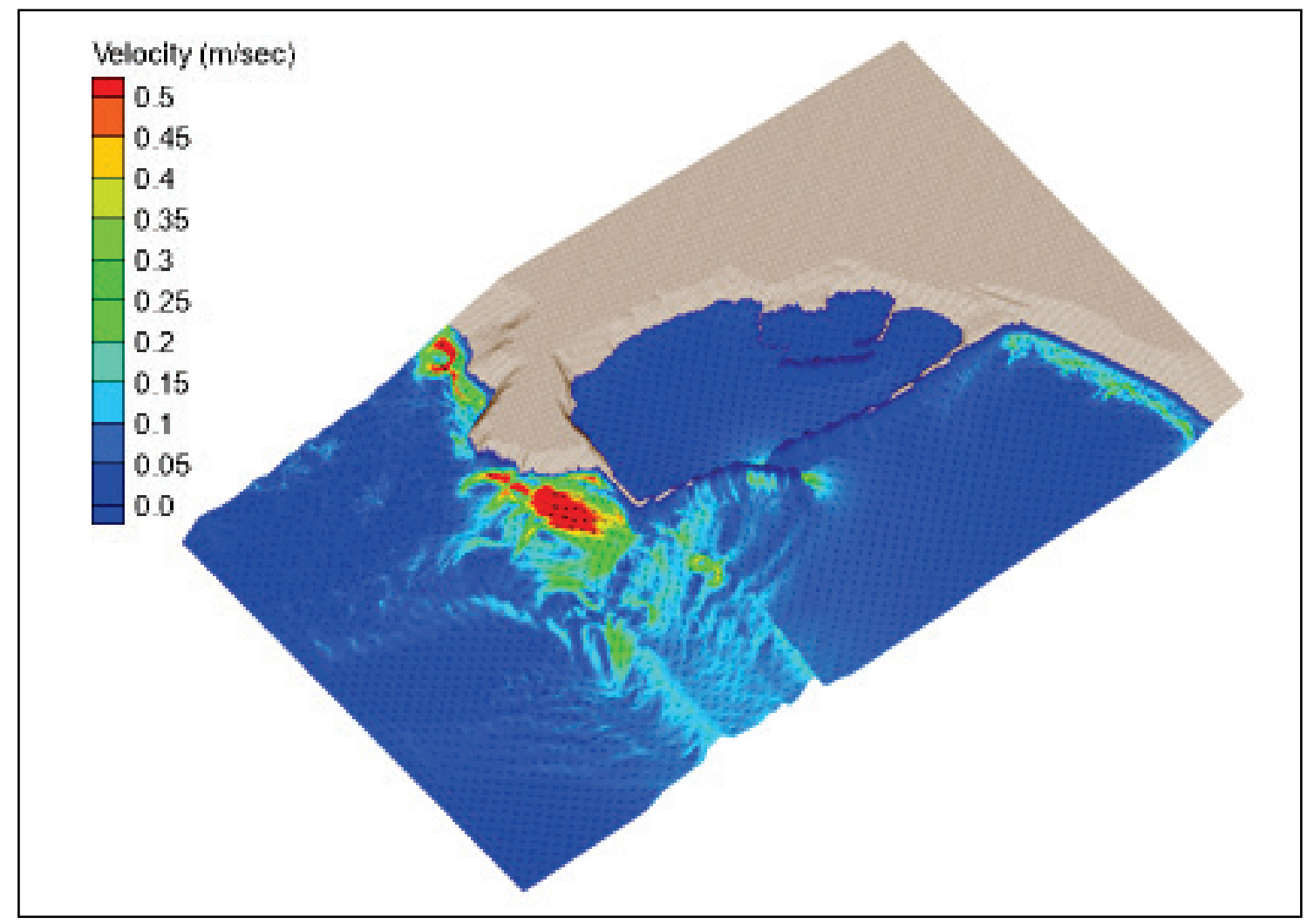


Figure B-2. Calculated wave fields by (a) BOUSS-2D, and (b) CMS-Wave at Point Judith Harbor, Rhode Island, for incident wave from SSE.

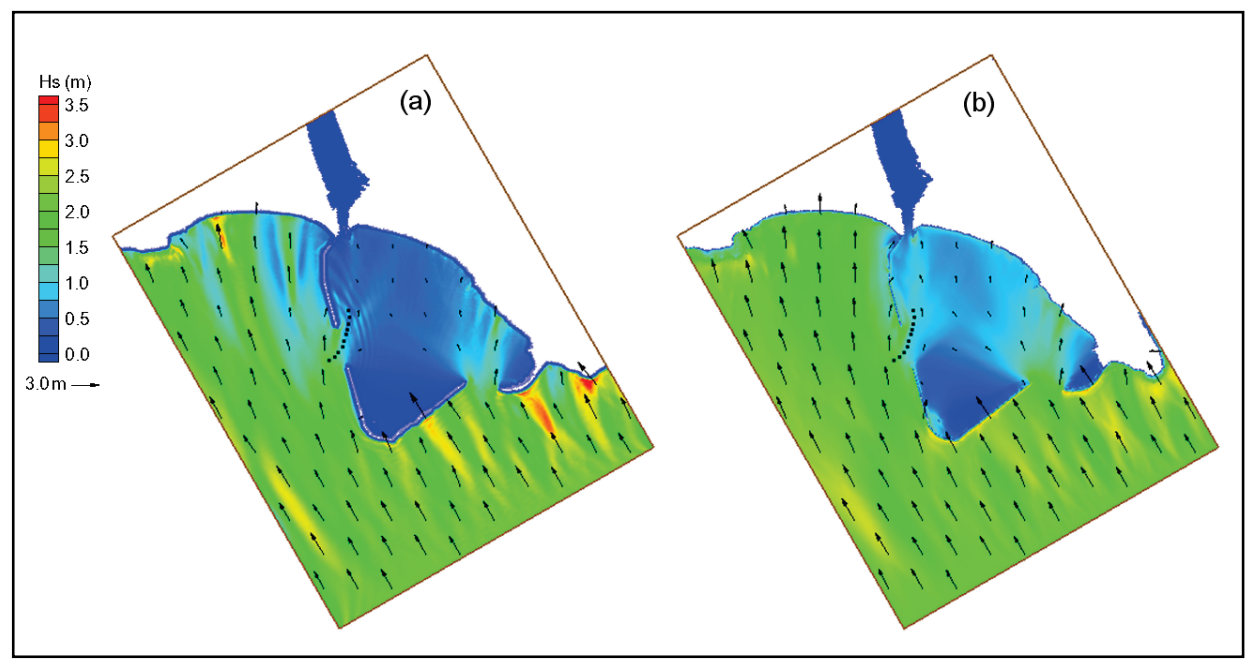

Figure B-3. Wave propagation inside a bay.

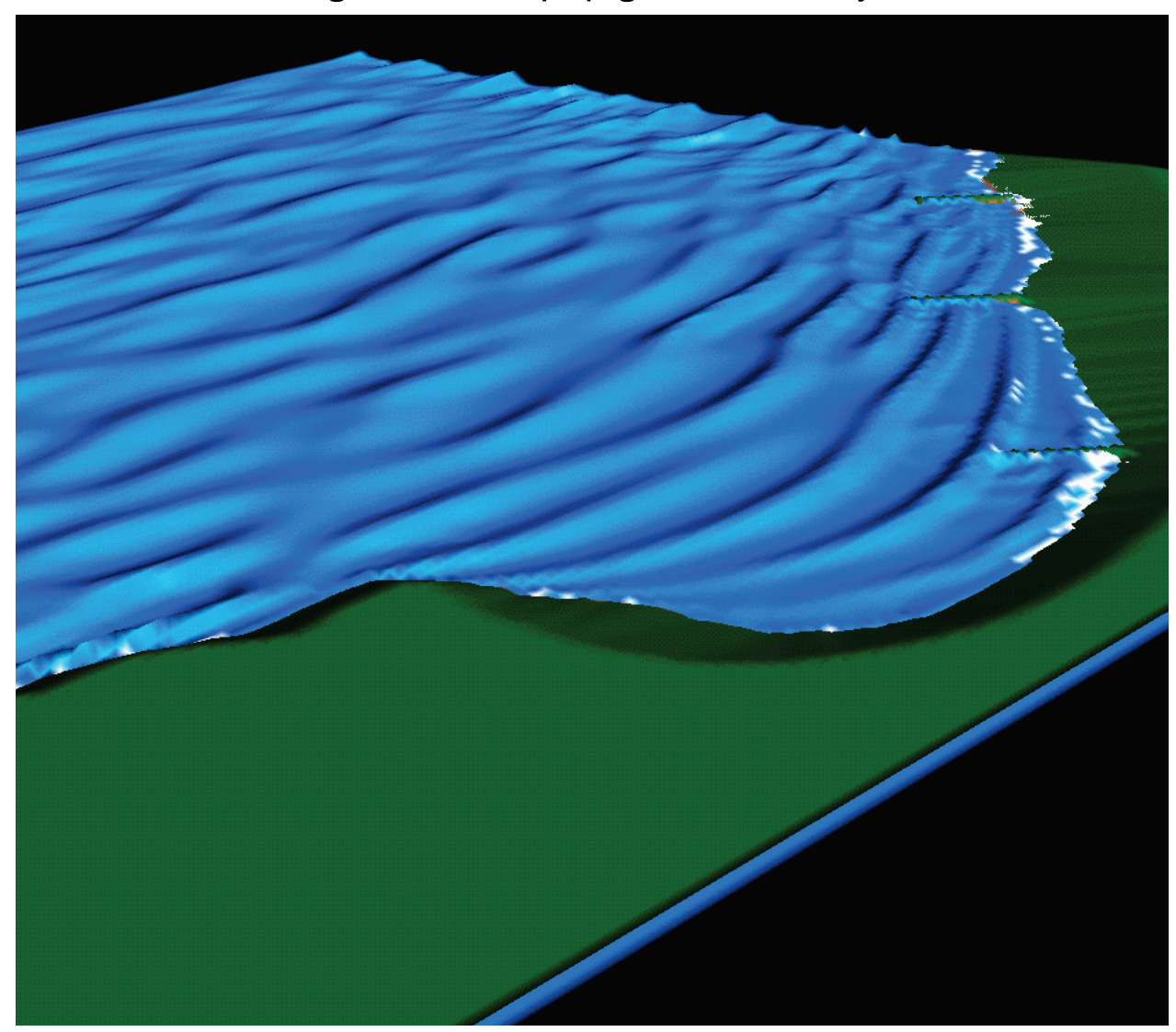


Figure B-4. Wave field around a detached breakwater.

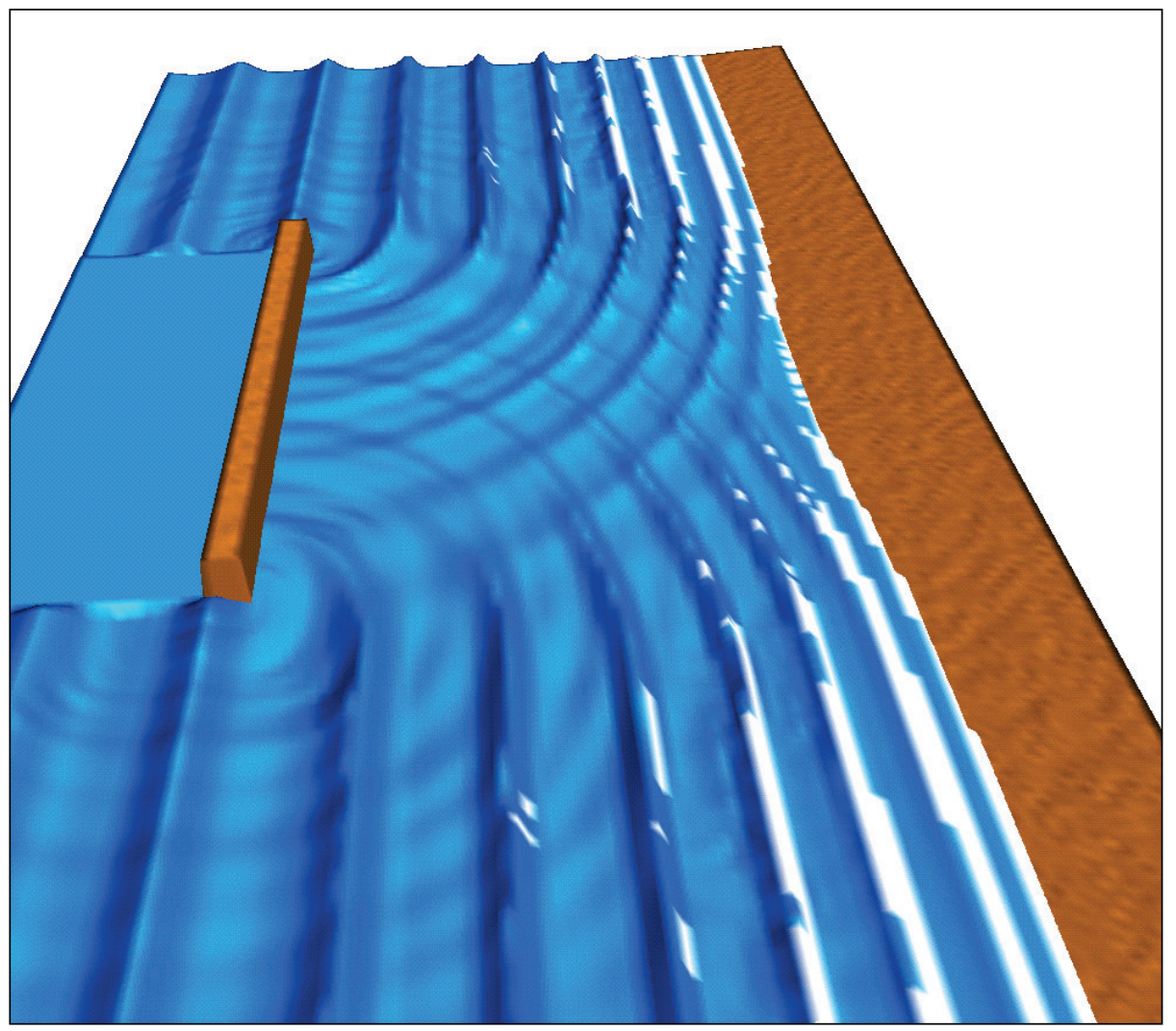

Figure B-5. Waves, wave-induced current, and circulation near a reflective jetty of an inlet.

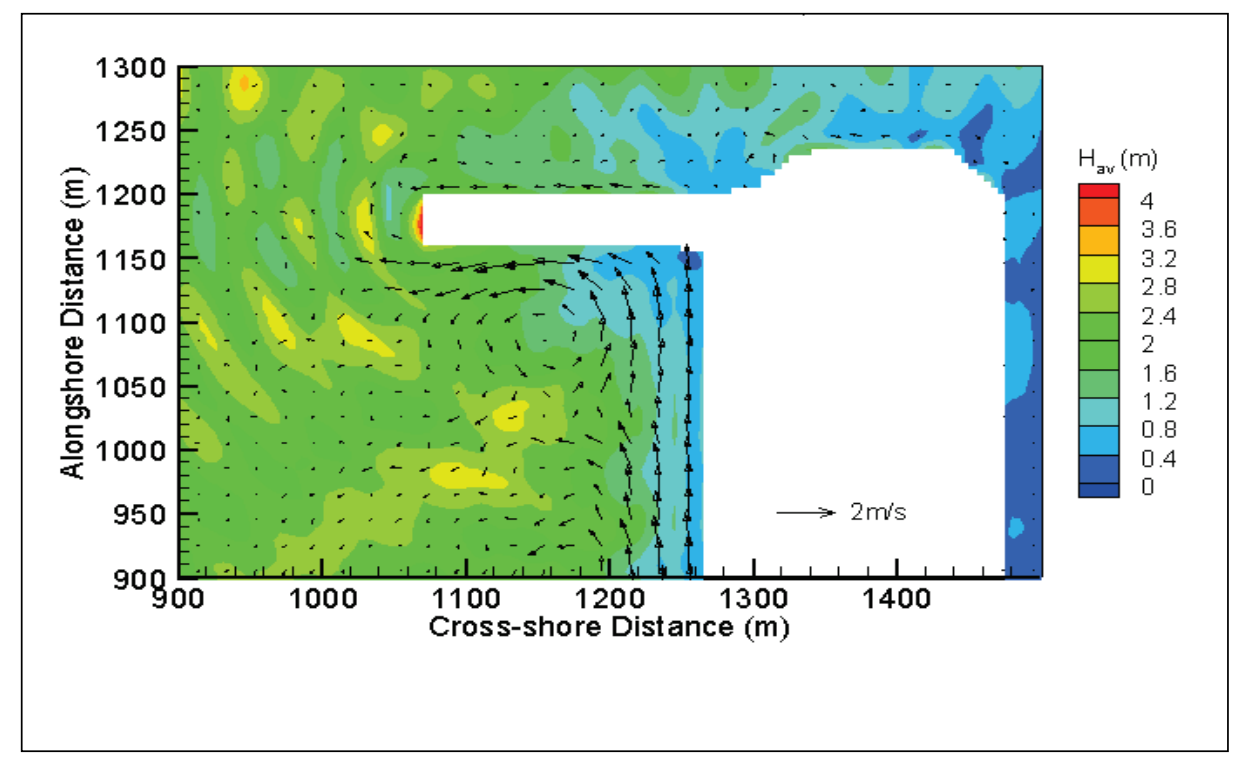


Figure B-6. Wave-induced current field developed between two groins placed on a beach.

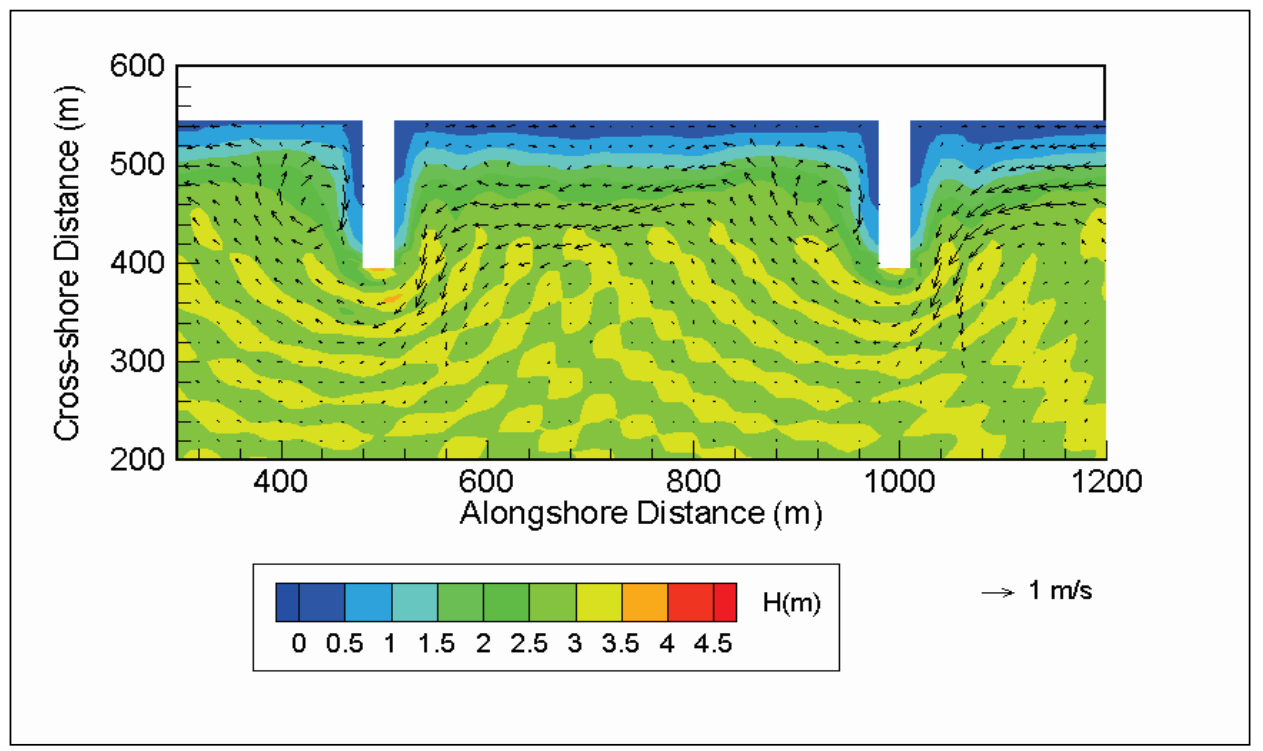

Figure B-7. Multiple ships moving (in transit) in a harbor.

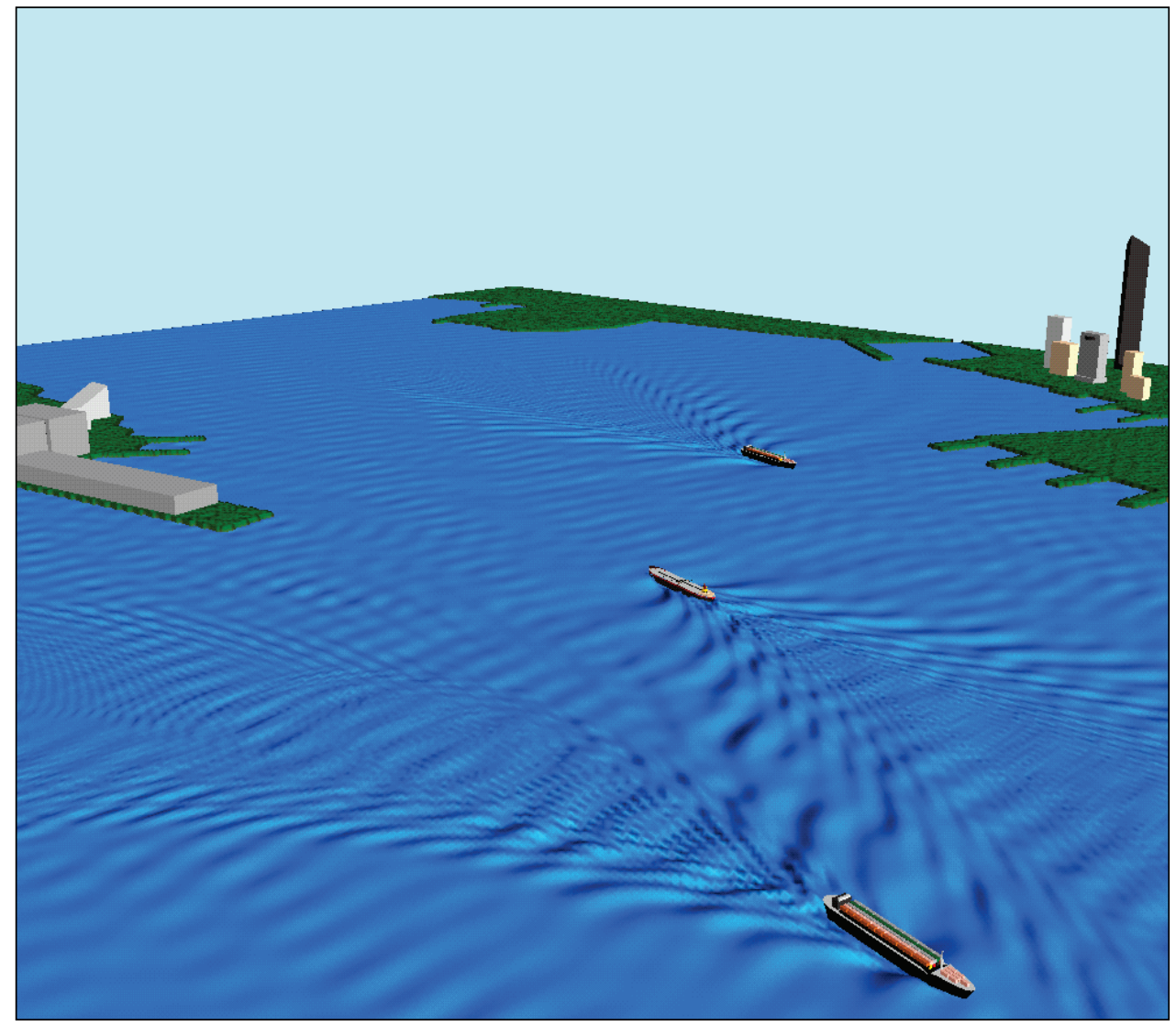


Figure B-8. BOUSS-2D domain for the Oyster Point Marina, California, entrance and east marina.

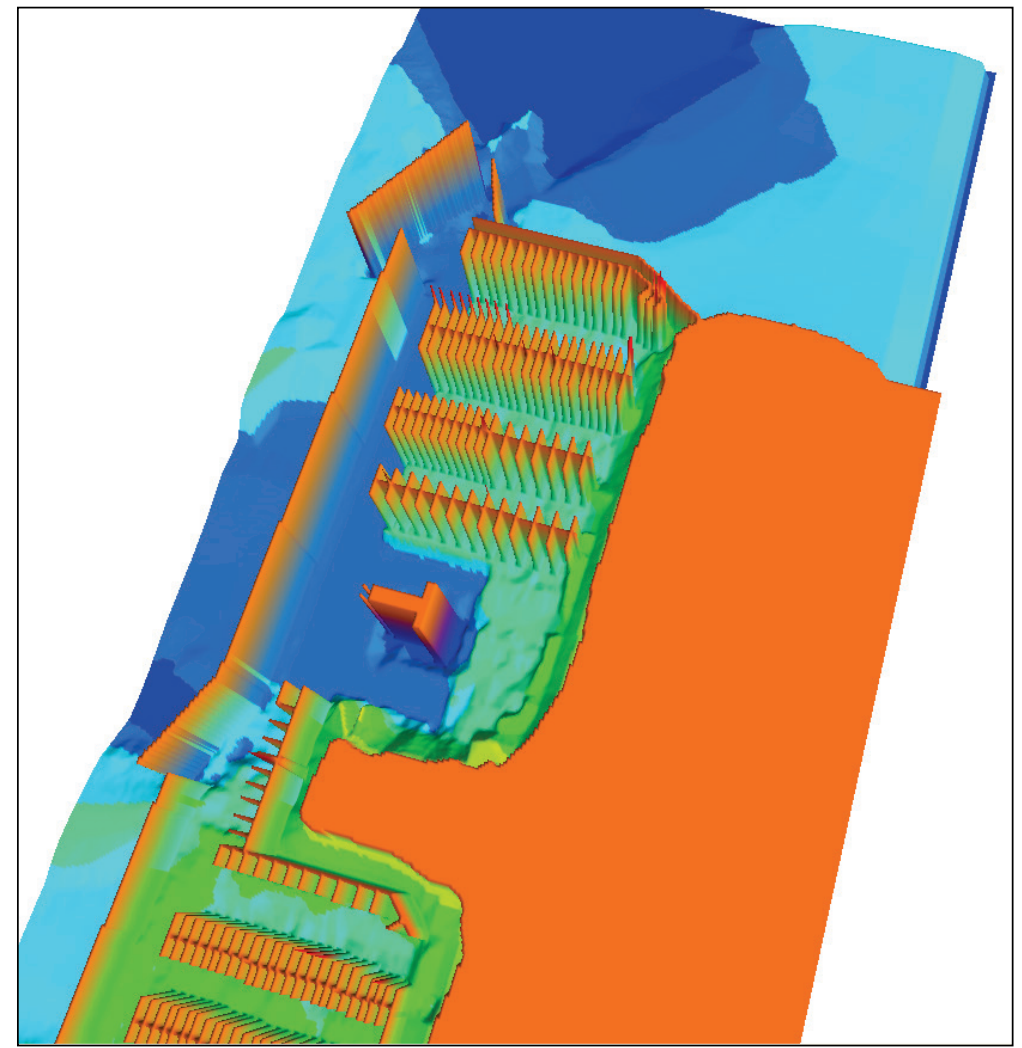

Figure B-9. BOUSS-2D grid for changes to entrance of Diversey Harbor, Michigan.

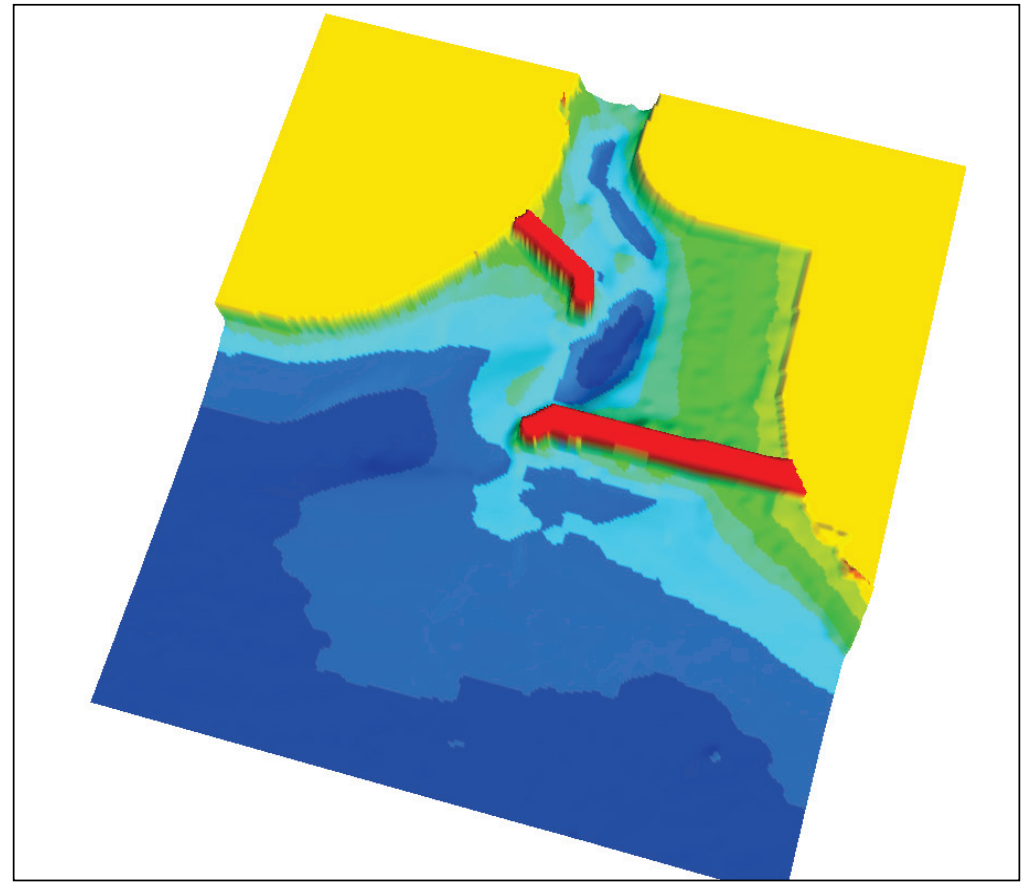


Figure B-10. BOUSS-2D runup/overtopping toolbox in SMS for a fringing reef application.

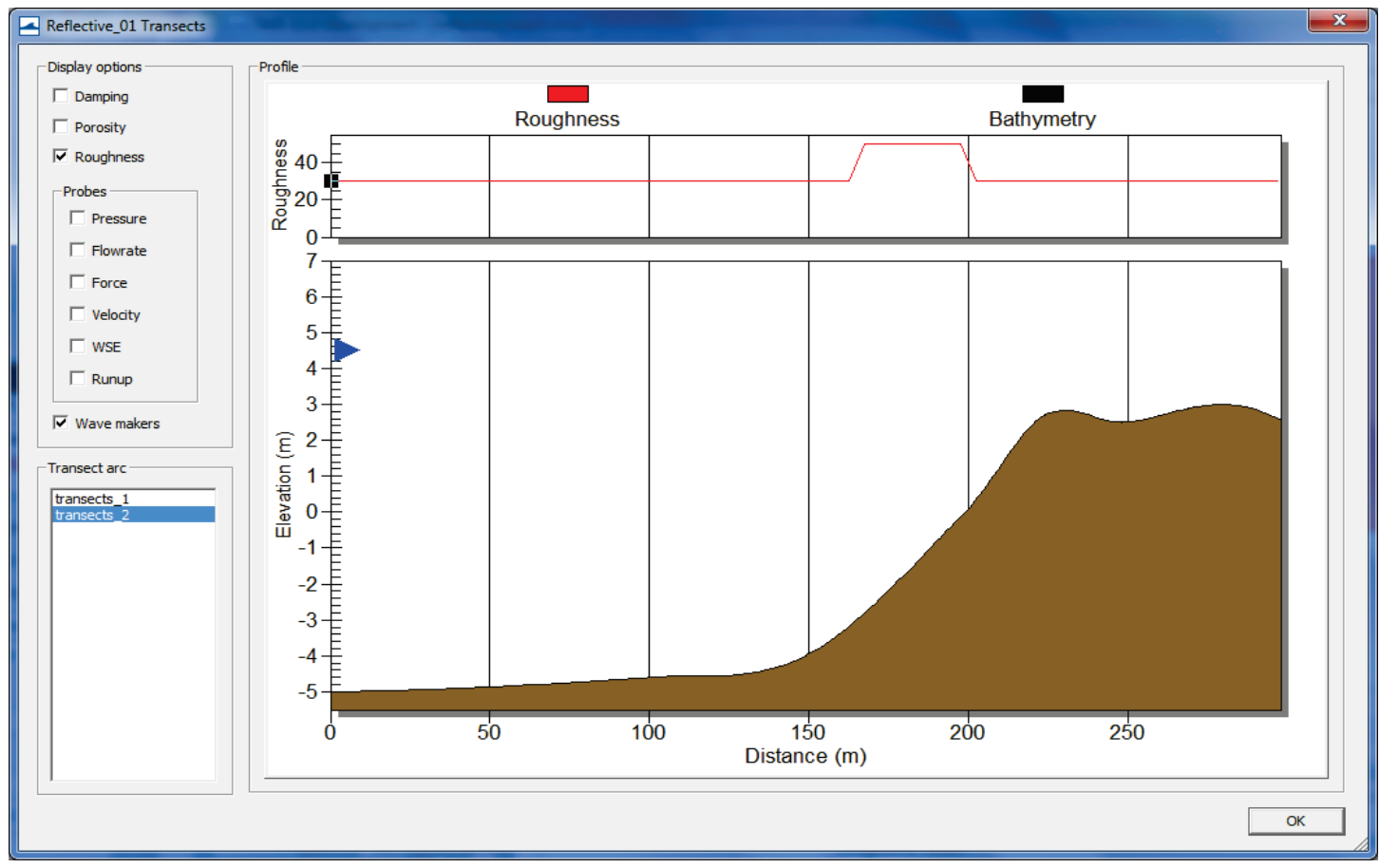




\section{Appendix C: Details of Metocean Data for CDF4}

Chapter 2 provides a summary of data used in this modeling study for the CDF4. This section of the report provides additional information about procedures used by LRB for developing meteorological and oceanographic (metocean) data for this numerical modeling study. The purpose of Appendix $\mathrm{C}$ is to document and provide details about the sources of metocean data and types of analyses used for the CDF4 study as well as for future studies in Lake Erie.

\section{C.1 Storm data for modeling}

In Chapter 2, three storms were selected for modeling wave overtopping of the CDF4. These included one hypothetical storm representing the 2-year wave and 2-year water level (Storm Condition 1), one hypothetical design storm representing the 20-year wave and 10-year water level (Storm Condition 2), and one actual storm (Storm Condition 3). The methodology and data used to identify, create, and assemble these three storms' data follow. For each storm, hourly data of the deepwater wave height, wave period, and wave direction, the wind speed and wind direction, and the water level are described.

\section{C.1.1 Data sources}

The wave and wind data offshore of the site are available from two sources. The WIS hourly wave hindcast conditions are available at 243 locations within Lake Erie for the 34-year period (1979-2012). The GLCFS provides hourly forecast wave data at user-selected locations in Lake Erie for the period of 2006 to present. As the WIS information spans a longer time period, which is more applicable for statistical analysis, the data from the WIS (Station 92243 - Figure C-1) will be used. Water elevation data at select U.S. locations on Lake Erie have been collected for over a century. Hourly water elevation data for Lake Erie near Buffalo are available from January 1960 to present.

\section{C.1.2 WIS data}

This section describes development of wave data for the hypothetical 2-year design storm (2-year wave and 2-year water level, Storm Condition 1), a 
hypothetical 20-year design storm (20-year wave and 10-year water level, Storm Condition 2), and an actual storm (Storm Condition 3). The three angle classes are defined as viewed by an observer on shore. Class Angle 1 represents the mean wave approach angle greater than 30 deg to the right of the normal from shore. Class Angle 2 represents the mean wave approach angle within 30 deg to either side of the normal from shore. Class Angle 3 represents the mean wave approach angle greater than 30 deg to the left of the normal from shore. Due to the limited fetch distance for Class Angle 1 and 3 , these will not be considered. Class Angle 2 waves encompass angles between $219 \mathrm{deg}$ and $279 \mathrm{deg}$ (Figure C-2). Table C-1 provides a list of storms during 1979-2012 with wave heights greater than $4 \mathrm{~m}$ (13.1 ft).

Figure C-1. Location of WIS Station $92243\left(42.80^{\circ} \mathrm{N},-78.96^{\circ} \mathrm{W}\right)$.

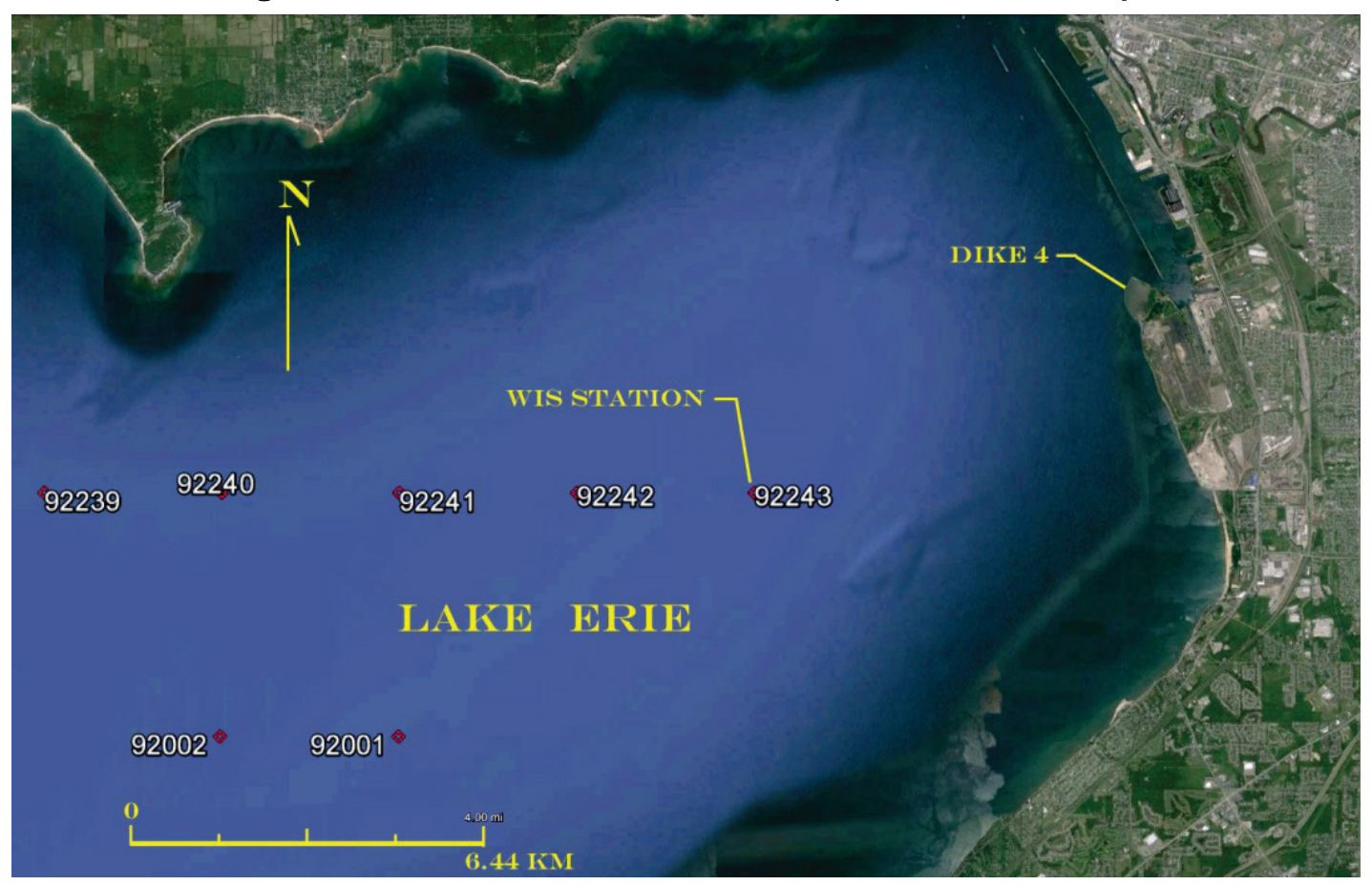


Figure C-2. Definition of Class Angle 2 wave directions.

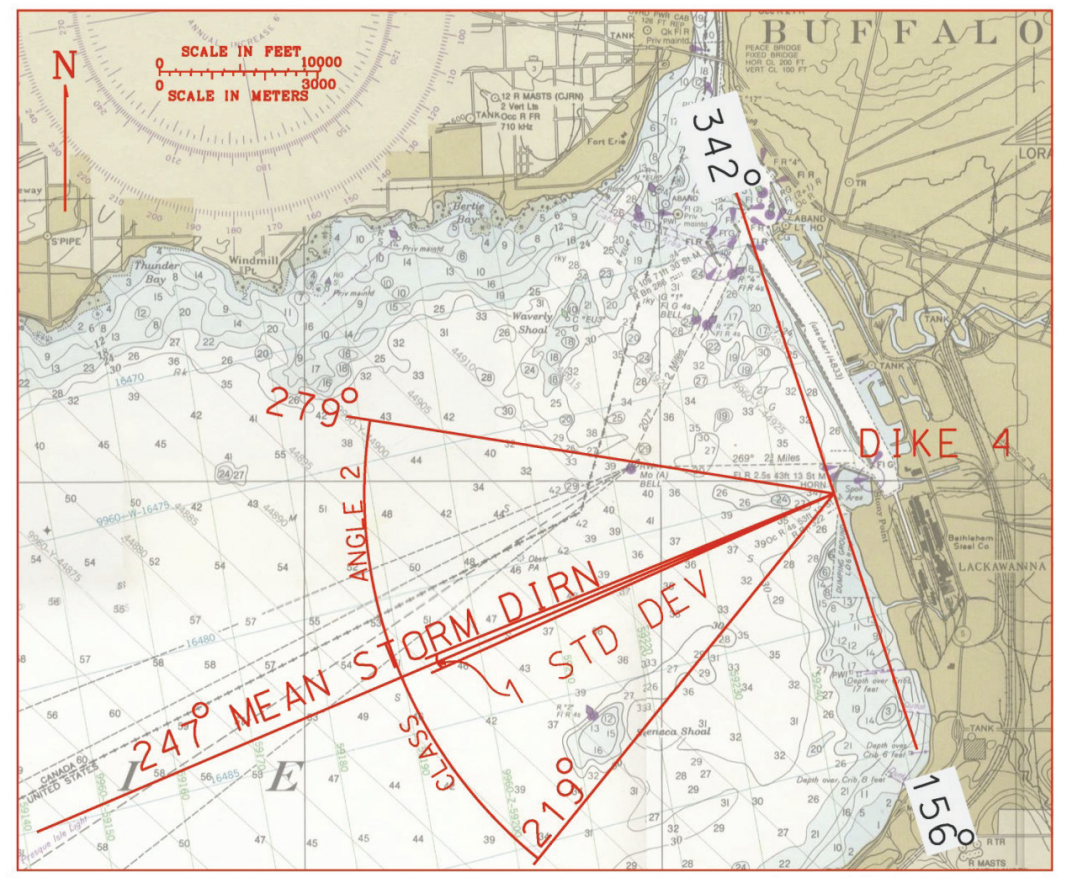

Table C-1. Storm list for wave heights greater than $4 \mathrm{~m}$ (13.1 ft) (1979-2012).

\begin{tabular}{|c|c|c|c|c|c|c|c|c|c|}
\hline Rank & Start Date & $\begin{array}{c}\text { Start } \\
\text { Time }\end{array}$ & End Date & $\begin{array}{c}\text { End } \\
\text { Time }\end{array}$ & Peak Date & $\begin{array}{c}\text { Peak } \\
\text { Time }\end{array}$ & $\begin{array}{c}\text { Veak } \\
\text { Value } \\
\text { (m) }\end{array}$ & $\begin{array}{c}\text { Tp } \\
\text { (sec) }\end{array}$ & $\begin{array}{c}\text { Direction } \\
\text { (deg) }\end{array}$ \\
\hline 1 & $2 / 24 / 2012$ & $19: 00$ & $2 / 26 / 2012$ & $7: 00$ & $2 / 25 / 2012$ & $1: 00$ & 4.85 & 9.73 & 249 \\
\hline 2 & $1 / 17 / 2012$ & $17: 00$ & $1 / 18 / 2012$ & $16: 00$ & $1 / 18 / 2012$ & $1: 00$ & 4.73 & 9.23 & 248 \\
\hline 3 & $2 / 1 / 2002$ & $15: 00$ & $2 / 2 / 2002$ & $6: 00$ & $2 / 1 / 2002$ & $20: 00$ & 4.72 & 10.13 & 247 \\
\hline 4 & $3 / 3 / 2012$ & $4: 00$ & $3 / 4 / 2012$ & $18: 00$ & $3 / 3 / 2012$ & $13: 00$ & 4.68 & 10.02 & 247 \\
\hline 5 & $1 / 28 / 2012$ & $14: 00$ & $1 / 30 / 2012$ & $17: 00$ & $1 / 28 / 2012$ & $22: 00$ & 4.68 & 9.35 & 248 \\
\hline 6 & $12 / 1 / 2006$ & $20: 00$ & $12 / 3 / 2006$ & $0: 00$ & $12 / 2 / 2006$ & $3: 00$ & 4.59 & 10.25 & 247 \\
\hline 7 & $1 / 1 / 2012$ & $16: 00$ & $1 / 3 / 2012$ & $5: 00$ & $1 / 2 / 2012$ & $3: 00$ & 4.57 & 10.04 & 247 \\
\hline 8 & $12 / 27 / 2008$ & $15: 00$ & $12 / 29 / 2008$ & $7: 00$ & $12 / 28 / 2008$ & $19: 00$ & 4.48 & 10.03 & 248 \\
\hline 9 & $10 / 14 / 2011$ & $16: 00$ & $10 / 18 / 2011$ & $10: 00$ & $10 / 15 / 2011$ & $21: 00$ & 4.37 & 10.15 & 248 \\
\hline 10 & $1 / 10 / 1982$ & $15: 00$ & $1 / 12 / 1982$ & $6: 00$ & $1 / 11 / 1982$ & $12: 00$ & 4.37 & 10.17 & 247 \\
\hline 11 & $12 / 1 / 1985$ & $20: 00$ & $12 / 3 / 1985$ & $13: 00$ & $12 / 2 / 1985$ & $13: 00$ & 4.36 & 10.03 & 247 \\
\hline 12 & $11 / 13 / 2003$ & $3: 00$ & $11 / 14 / 2003$ & $10: 00$ & $11 / 13 / 2003$ & $14: 00$ & 4.33 & 10.92 & 247 \\
\hline 13 & $1 / 4 / 1982$ & $12: 00$ & $1 / 5 / 1982$ & $21: 00$ & $1 / 5 / 1982$ & $3: 00$ & 4.3 & 10.84 & 246 \\
\hline 14 & $12 / 9 / 2009$ & $14: 00$ & $12 / 12 / 2009$ & $12: 00$ & $12 / 10 / 2009$ & $5: 00$ & 4.29 & 10.78 & 246 \\
\hline 15 & $12 / 15 / 1987$ & $14: 00$ & $12 / 17 / 1987$ & $6: 00$ & $12 / 16 / 1987$ & $8: 00$ & 4.27 & 10.97 & 246 \\
\hline
\end{tabular}




\begin{tabular}{|c|c|c|c|c|c|c|c|c|c|}
\hline Rank & Start Date & $\begin{array}{c}\text { Start } \\
\text { Time }\end{array}$ & End Date & $\begin{array}{c}\text { End } \\
\text { Time }\end{array}$ & Peak Date & $\begin{array}{c}\text { Peak } \\
\text { Time }\end{array}$ & $\begin{array}{c}\text { Peak } \\
\text { Value } \\
\text { (mo })\end{array}$ & $\begin{array}{c}\text { Tp } \\
(\mathbf{s e c})\end{array}$ & $\begin{array}{c}\text { Direction } \\
\text { (deg) }\end{array}$ \\
\hline 16 & $12 / 17 / 2000$ & $15: 00$ & $12 / 18 / 2000$ & $20: 00$ & $12 / 18 / 2000$ & $3: 00$ & 4.22 & 9.98 & 247 \\
\hline 17 & $11 / 6 / 1990$ & $3: 00$ & $11 / 6 / 1990$ & $23: 00$ & $11 / 6 / 1990$ & $11: 00$ & 4.15 & 10.42 & 247 \\
\hline 18 & $4 / 28 / 2011$ & $11: 00$ & $4 / 29 / 2011$ & $3: 00$ & $4 / 28 / 2011$ & $16: 00$ & 4.11 & 9.19 & 246 \\
\hline 19 & $1 / 9 / 2008$ & $5: 00$ & $1 / 10 / 2008$ & $5: 00$ & $1 / 9 / 2008$ & $13: 00$ & 4.11 & 8.48 & 249 \\
\hline 20 & $2 / 5 / 2006$ & $7: 00$ & $2 / 7 / 2006$ & $12: 00$ & $2 / 5 / 2006$ & $14: 00$ & 4.1 & 9.63 & 246 \\
\hline 21 & $1 / 8 / 1989$ & $4: 00$ & $1 / 9 / 1989$ & $23: 00$ & $1 / 8 / 1989$ & $20: 00$ & 4.06 & 9.73 & 248 \\
\hline 22 & $1 / 13 / 2012$ & $8: 00$ & $1 / 14 / 2012$ & $11: 00$ & $1 / 13 / 2012$ & $15: 00$ & 4.01 & 9.2 & 247 \\
\hline
\end{tabular}

\section{C.1.3 Wave height-recurrence interval}

The significant wave height recurrence interval (return period) information for the Class Angle 2 was developed by sorting and ranking all storms for the period of record over $1 \mathrm{~m}$ (Figure $\mathrm{C}-3$ ). The best-fit curve for the waves above $4 \mathrm{~m}(13.1 \mathrm{ft})$ is defined as

$$
H_{m o}=0.2805 \ln (R I)+4.006
$$

where $H_{m o}=$ wave height at WIS Station 92243 in meters and RI = "Recurrence Interval" or "Return Period" of wave in years.

Figure C-3. Wave height vs. return period.

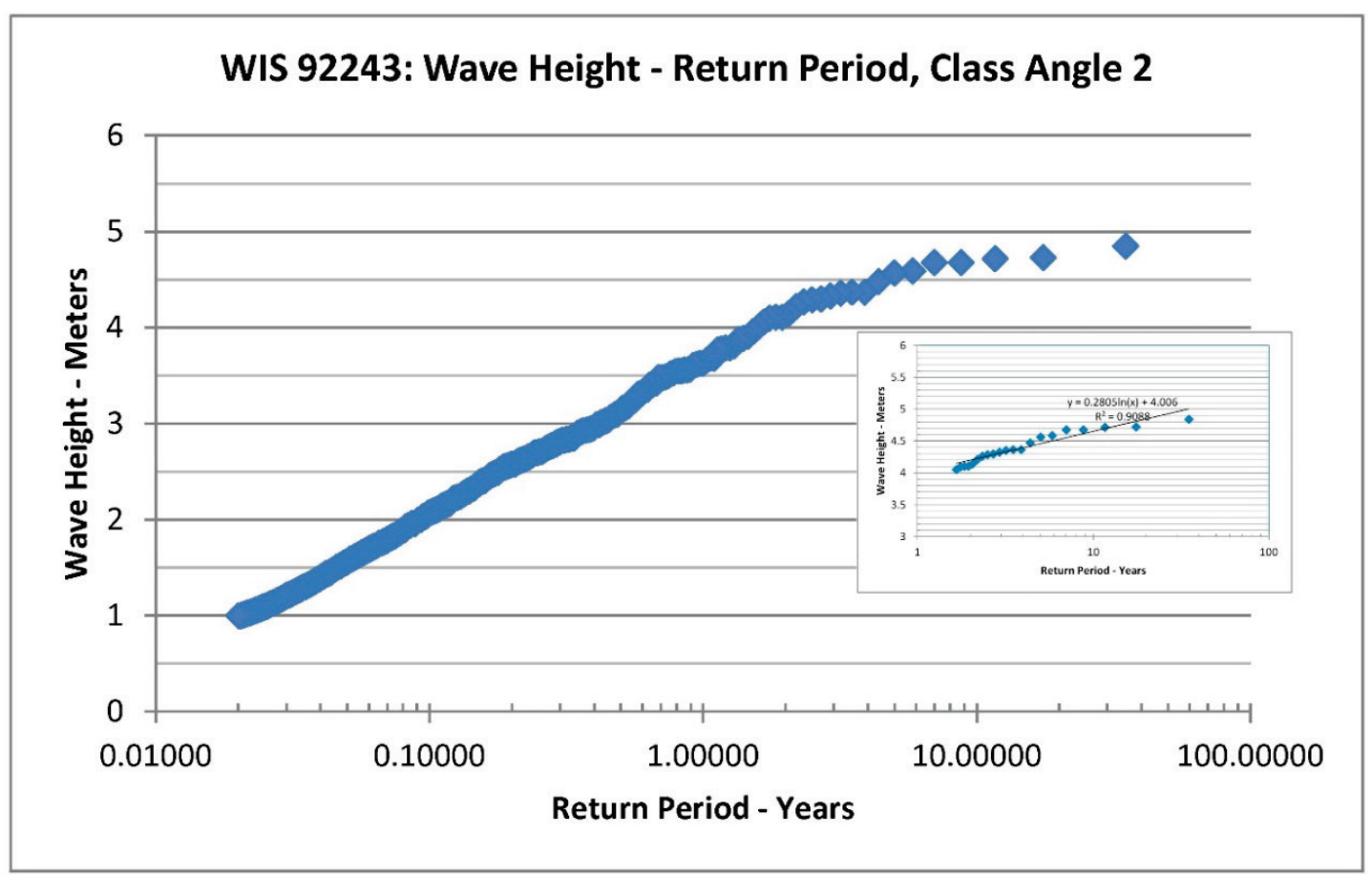




\section{C.1.4 Wave Period}

The corresponding wave period was identified by plotting all storm wave heights $\left(\mathrm{H}_{\mathrm{mo}}>1 \mathrm{~m}\right)$ and wave periods within the Class Angle 2 (Figure $\left.\mathrm{C}-4\right)$. The best-fit curve to data is given by

$$
T_{p}=-0.1407 H_{m o}^{2}+2.193 H_{m o}+3.0877
$$

Figure C-4. Wave period vs. wave height relation.

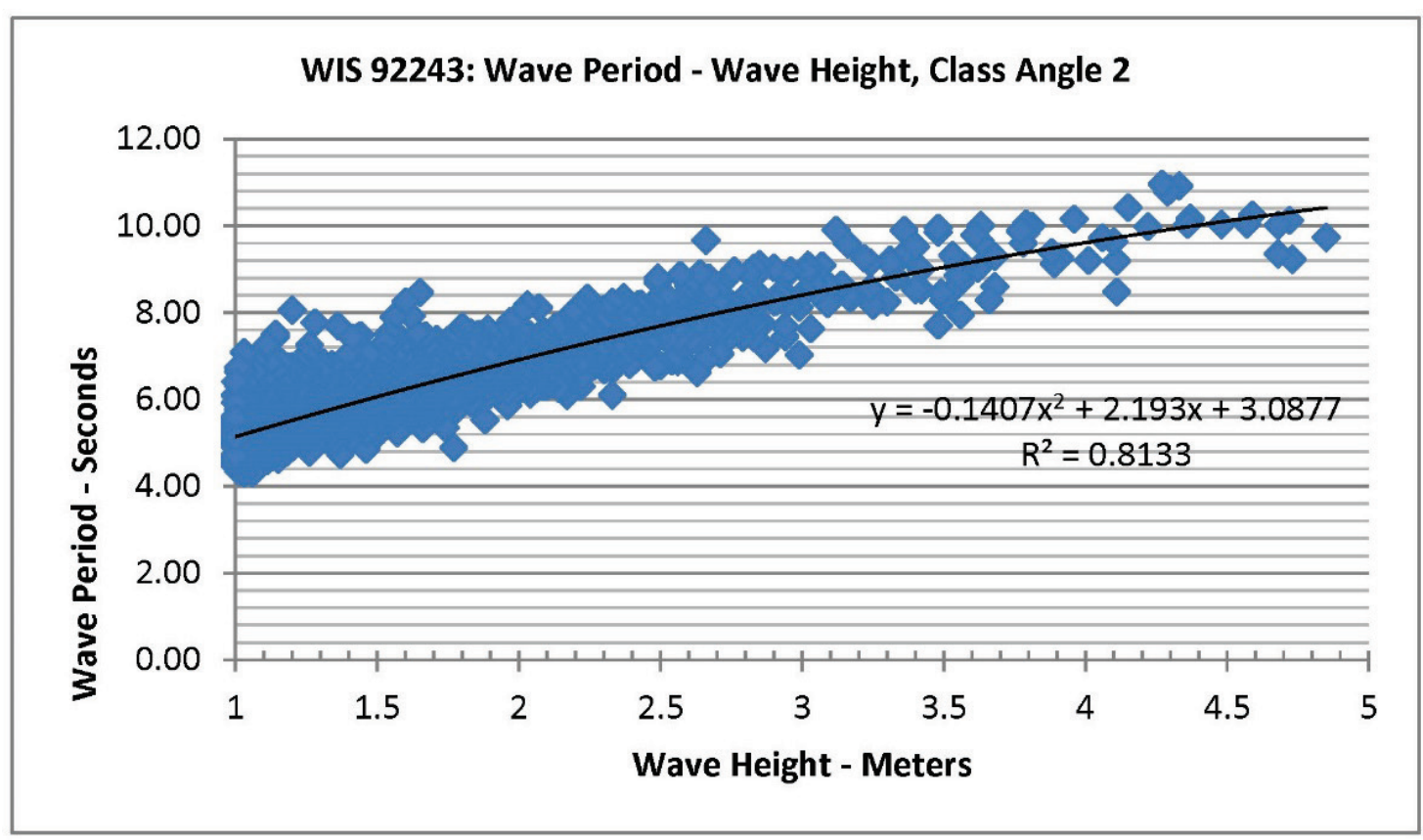

\section{C.1.5 Wave direction distribution}

The descriptive statistics for the direction of all peak storm waves greater than $4 \mathrm{~m}(13.1 \mathrm{ft}), 3 \mathrm{~m}(9.8 \mathrm{ft})$, and $2 \mathrm{~m}(6.6 \mathrm{ft})$ were determined and presented in Table C-2. It was found that the mean wave direction for all major storms (greater than $4 \mathrm{~m}[13.1 \mathrm{ft}]$ ) approached from 247 deg azimuth with a standard deviation of approximately $1 \mathrm{deg}$ (Figure C-2).

Table C-2. Descriptive statistics for storm wave direction.

\begin{tabular}{|l|c|c|c|}
\hline \multirow{2}{*}{$\begin{array}{l}\text { Descriptive } \\
\text { Statistic }\end{array}$} & \multicolumn{3}{|c|}{ Wave Direction - Degrees } \\
\cline { 2 - 4 } & $\begin{array}{c}\text { Waves above 4 } \\
\mathbf{m}\end{array}$ & $\begin{array}{c}\text { Waves above 3 } \\
\mathbf{m}\end{array}$ & $\begin{array}{c}\text { Waves above 2 } \\
\mathbf{m}\end{array}$ \\
\hline Mean & 247.2 & 247.4 & 246.2 \\
\hline Standard error & 0.193 & 0.131 & 0.689 \\
\hline Median & 247 & 247 & 248 \\
\hline
\end{tabular}




\begin{tabular}{|l|c|c|c|}
\hline \multirow{2}{*}{$\begin{array}{l}\text { Descriptive } \\
\text { Statistic }\end{array}$} & \multicolumn{3}{|c|}{ Wave Direction - Degrees } \\
\cline { 2 - 4 } & $\begin{array}{c}\text { Waves above 4 } \\
\mathrm{m}\end{array}$ & $\begin{array}{c}\text { Waves above 3 } \\
\mathrm{m}\end{array}$ & $\begin{array}{c}\text { Waves above 2 } \\
\mathrm{m}\end{array}$ \\
\hline $\begin{array}{l}\text { Standard } \\
\text { deviation }\end{array}$ & 0.907 & 1.190 & 247 \\
\hline Sample variance & 0.823 & 1.416 & 13.617 \\
\hline Kurtosis & -0.308 & 0.211 & 234.494 \\
\hline Skewness & 0.453 & 0.576 & -13.883 \\
\hline Range & 3 & 6 & 262 \\
\hline Minimum & 246 & 245 & 10 \\
\hline Maximum & 249 & 251 & 272 \\
\hline Sum & 5438 & 20535 & 96283 \\
\hline Count & 22 & 83 & 391 \\
\hline
\end{tabular}

\section{C.2 Data for the 2-year and 20-year storms}

Table C-3 lists the wave recurrence interval information for the Class Angle 2 for the WIS station data.

Table C-3. Class Angle 2 recurrence interval for WIS Sta 92243.

\begin{tabular}{|c|c|c|}
\hline RI - Years & $\mathbf{H}_{\text {mo }} \mathbf{M}$ & Tp Sec \\
\hline 1 & 3.6 & 9.2 \\
\hline 2 & 4.2 & 9.8 \\
\hline 5 & 4.5 & 10.1 \\
\hline 10 & 4.7 & 10.2 \\
\hline 20 & 4.8 & 10.4 \\
\hline 50 & 5.1 & 10.6 \\
\hline
\end{tabular}

\section{C.2.1 Storm selection}

Three storms were used to create the typical storm pattern hydrographs for the 2-year and the 20-year waves based upon the events listed in Table C-1, which had peak wave height values close to those in Table C-3. For the actual storm, an event was selected from the storm list (Table C-1) that was not used in creating the synthetic storms, was relatively frequent (3-5 years) and had a similar water level recurrence interval. The water level-frequency will be discussed subsequently. 
For the 2-year storm, three storms from Table $\mathrm{C}-1$ had a wave height vs. return period near 2-years. These include storms on 18 December 2000, 6 November 1990, and 28 April 2011. Three storms with the wave height-return period for the 20-year storms occurred on 25 December 2013, 18 January 2012, and 1 February 2002. Four storms were considered for the actual storm, which had a wave height recurrence interval between 3 to 5 years. These were storms on 15 October 2011 (3.5-year wave, $\sim$-year water level), 11 January 1982 (3.5-year wave, 3-year water level), 2 December 1985 (3.2-year wave, water level highest for period of record), and 11 November 2003 (2.9-year wave, 1-year water level). The 11 January 1982 storm was selected as the water level associated with this event demonstrated a peak elevation with a similar recurrence interval.

\section{C.2.2 Wave height, period and direction data}

Wave Height. The wave height hydrograph for the 2-year and the 20-year synthetic storms were created by plotting the three storms, adjusting the timing so all peaks align at same time, averaging the three storm wave heights, and then finally adjusting all wave heights based on ratio of average wave height at peak and the determined wave height for the 2-year or 20-year storm. Figures C- 5 and C- 6 present the three storms used to create the pattern hydrographs for the 2-year and 20-year wave, respectively.

Figure $\mathrm{C}-13$ to Figure $\mathrm{C}-15$ present the wave height hydrographs for the 2-year wave and 2-year water level event, the 20-year wave and 10-year water level event, and the 11 January 1982 storm, respectively. 
Figure C-5. Three storms used for 2-year wave height hydrograph (peaks aligned).

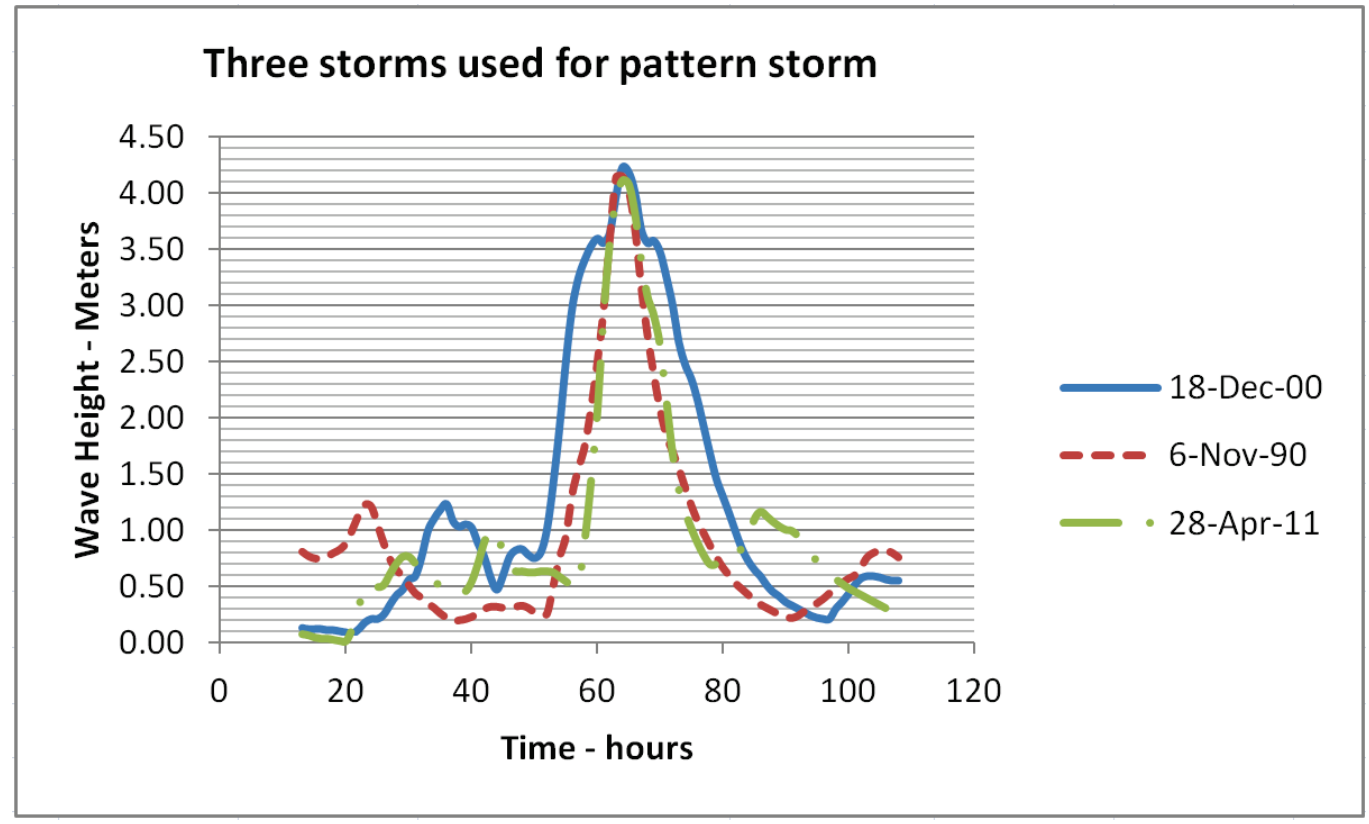

Figure C-6. Three storms used for 20-year wave height hydrograph (peaks aligned).

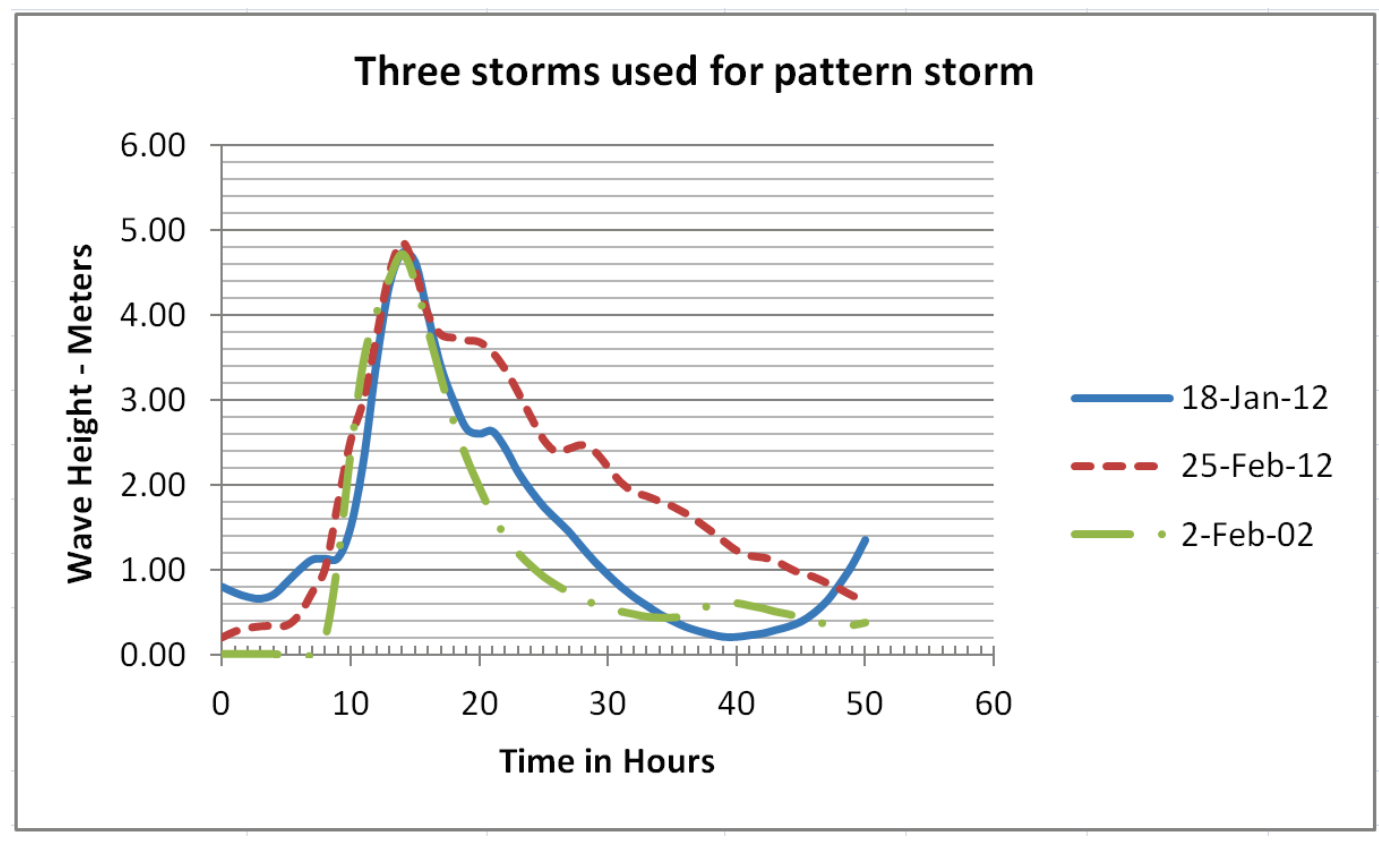

Wave Period. To develop the wave period pattern hydrograph, the three storms were plotted, the timing was adjusted as was done for the wave heights, the three storm wave period graphs were averaged, and the period adjusted by the ratio of the average wave period that occurs at the time of the peak wave height to the determined wave period for that frequency storm as presented in Table C-3. Note that the highest wave period did not occur at the same time as the highest wave, but usually afterwards (1-2 hr). 
Figure C-7 and Figure C-8 present the three storms used to create the pattern hydrographs for the 2-year and 20-year wave, respectively. Figures $\mathrm{C}-13$ to $\mathrm{C}-15$ present the wave period graphs for the 2-year wave and 2-year water level event, the 20-year wave and 10-year water level event, and the 11 January 1982 storm, respectively.

Figure C-7. Three storms used for 2-year wave period hydrograph (peaks aligned).

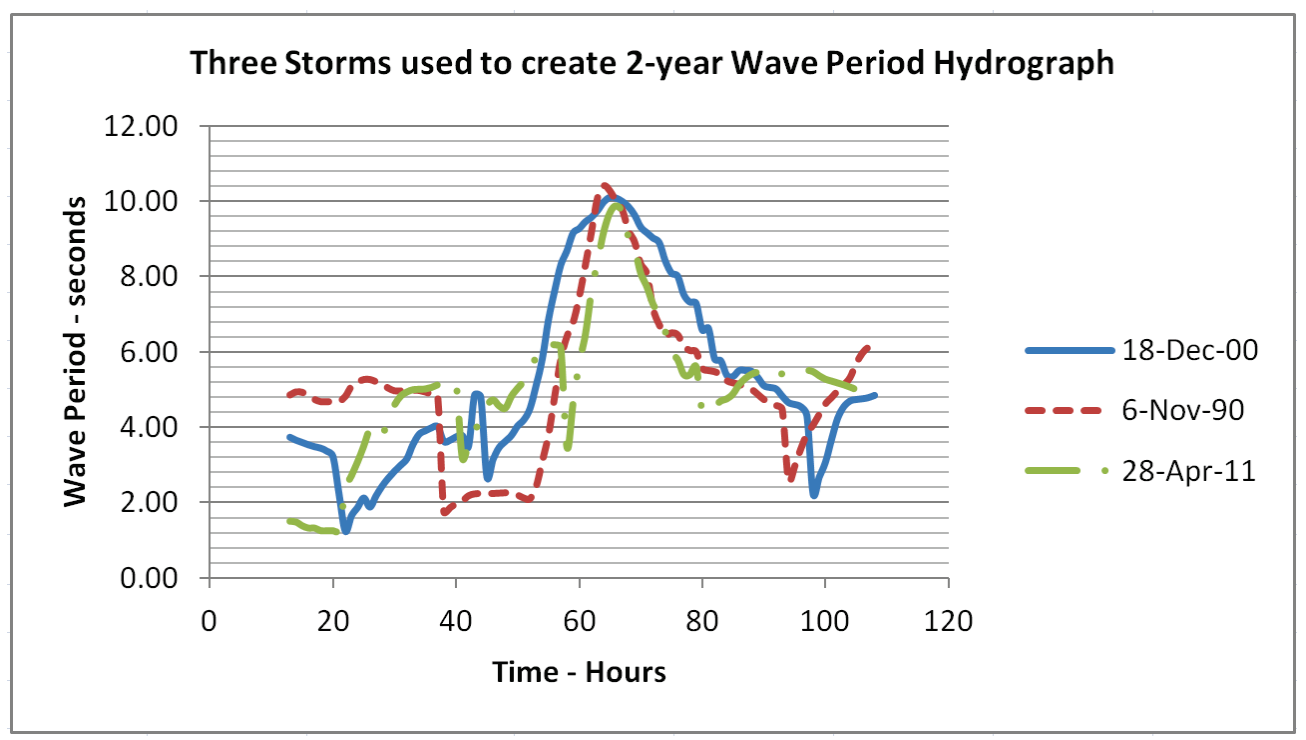

Figure C-8. Three storms used for 20-year wave period hydrograph (peaks aligned).

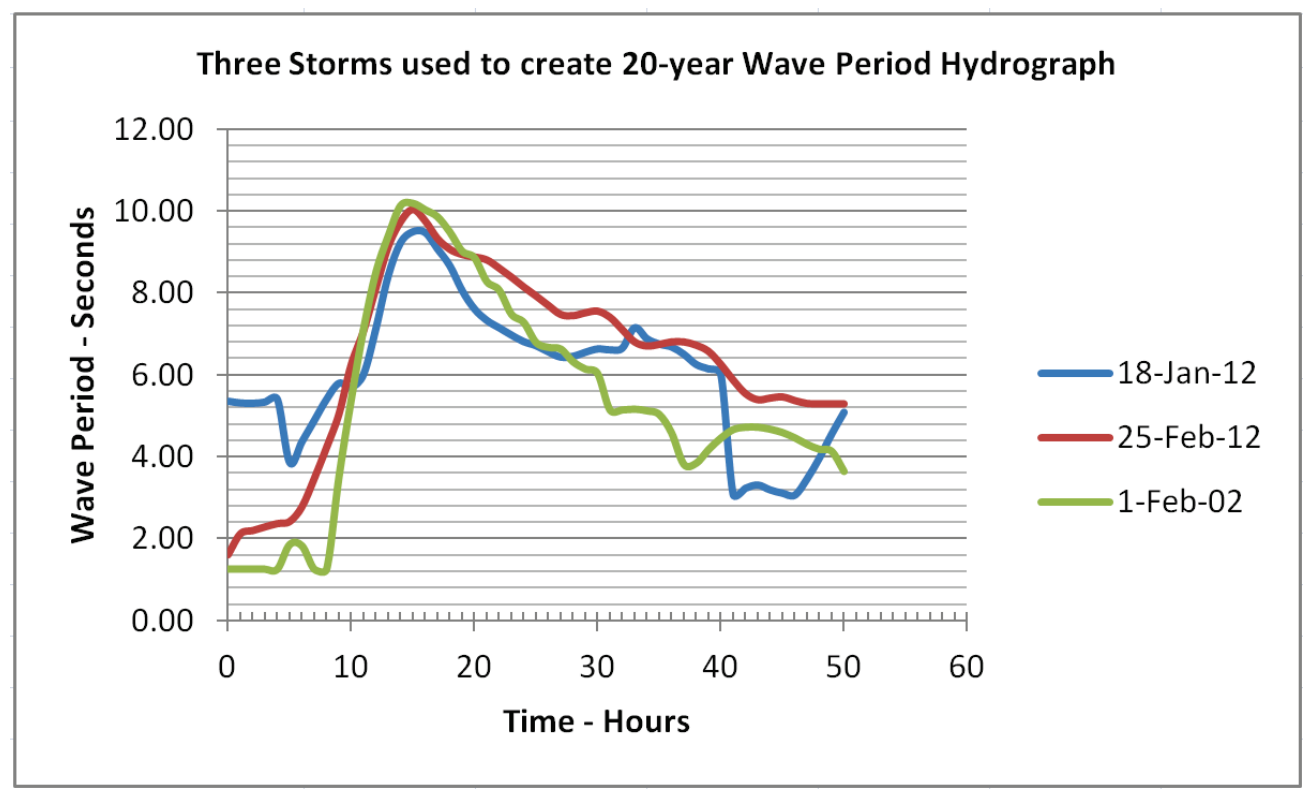

Direction. A constant wave direction of $247 \mathrm{deg}$ was used for the synthetic storms based upon the descriptive statistics presented in Table C-2, and the actual values were used for the 11 January 1982 storm. 


\section{C.2.3 Wind speed}

Some of the factors that affect the generation of waves are the wind speed, wind duration, fetch, water depth, bed roughness, water temperature, and air temperature. While the actual wind speed will be used for the "actual storm" modeled, a simple relation was created to select the wind speed corresponding with the waves for the synthetic storms. Using the WIS hourly data, waves from 247 deg were plotted with the accompanying wind speed. The following best-fit equation was developed and was used to generate a wind speed hydrograph to accompany the synthetic storms:

$$
\text { Wind Speed }=4.2957 H_{m o}+2.5385
$$

Figure C-14 illustrates the adopted wave height-wind speed relation. Figures $\mathrm{C}-13$ to $\mathrm{C}-15$ present the wind speed graphs for the 2-year wave and 2-year water level event, the 20-year wave and 10-year water level event, and the 11 January 1982 storm, respectively.

Figure C-9. Wave height vs.wind speed at constant wind direction of 247 deg.

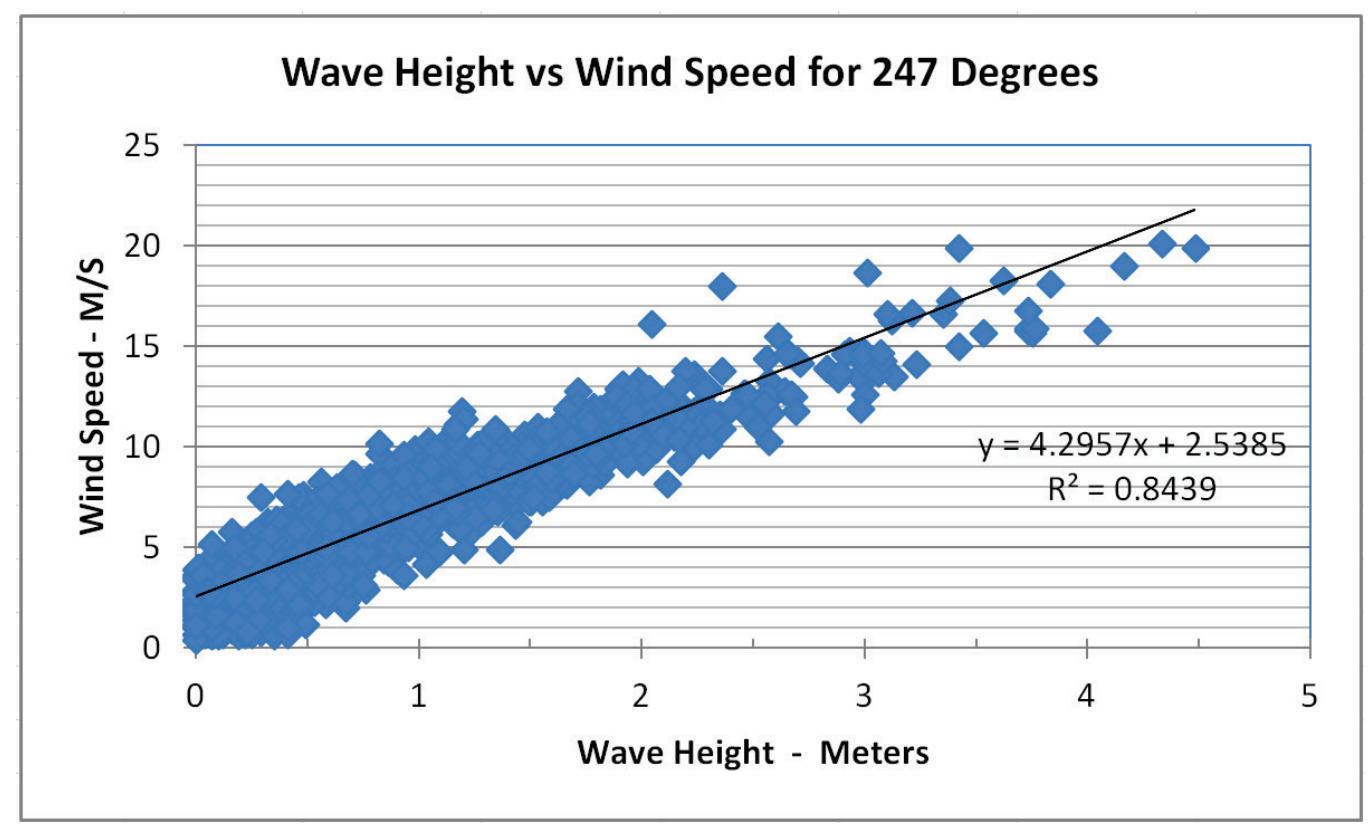

\section{C.2.4 Wind direction}

A constant wind direction of 247 deg was used for the synthetic storms (same as used for the wave direction), and the actual values were used for the 11 January 1982 storm. 


\section{C.2.5 Water level}

The NOAA maintains a water level gage at Buffalo Harbor. Station 9063020 is located on the south side of the Buffalo River, near the upriver end of the U.S. Coast Guard base, $42^{\circ}-52.6^{\prime} \mathrm{N}$ and $78^{\circ}-53.4^{\prime} \mathrm{W}$. Monthly water level data are available in digital form since 1860 , with daily and hourly water level data available in digital form since January 1960. Annual maximum and minimum water levels are available in digital form since 1900 . The lake level recurrence interval (return period) information was developed by sorting and ranking all annual maximum water levels for the period of record of 1900-2014 (Figure C-10). The 2-year, 10-year, 20-year, and 50-year lake levels are listed in Table $\mathrm{C}-4$.

Table C-4. Return period for lake level.

\begin{tabular}{|c|c|}
\hline Return Period - Years & Water Level - M IGLD 1985 \\
\hline 2 & 175.80 \\
\hline 10 & 176.45 \\
\hline 20 & 176.56 \\
\hline 50 & 176.74 \\
\hline
\end{tabular}

Figure C-10. Lake Erie water elevation at Buffalo, NY (Return Period relation).

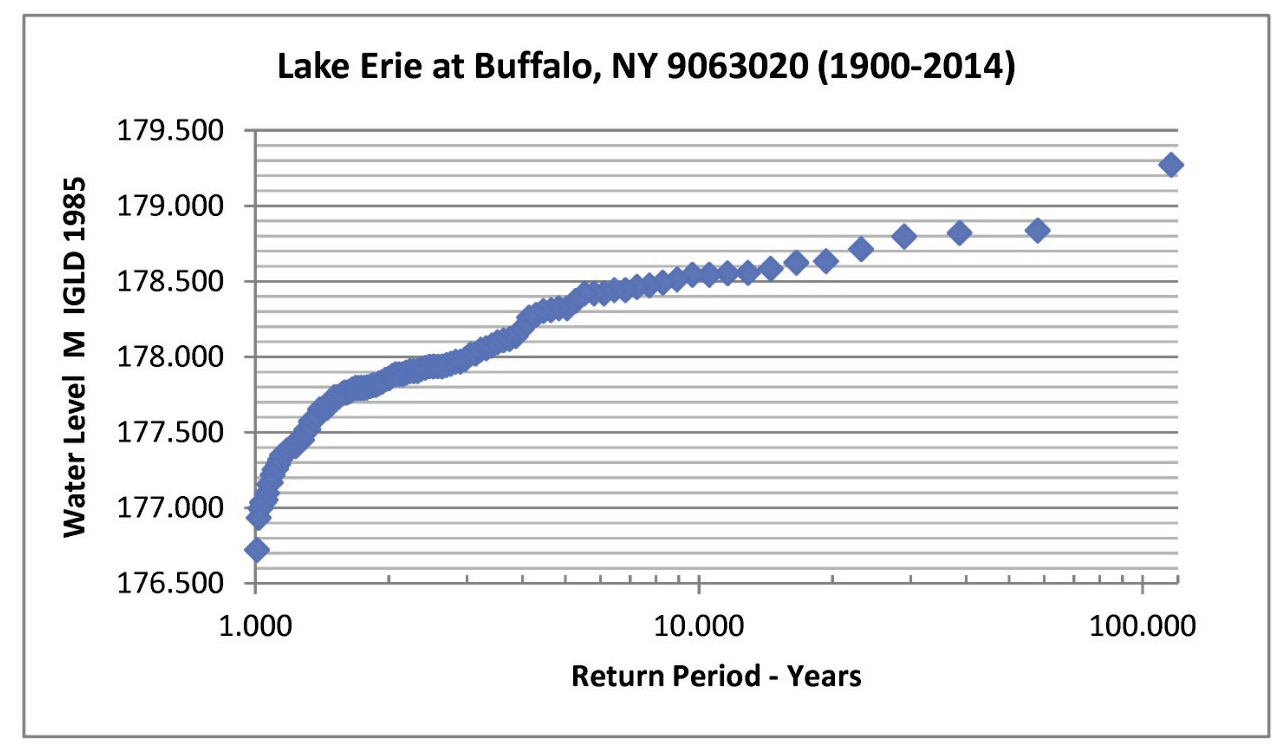

\section{C.2.6 Storm hydrographs}

Three storms were used to create the typical storm pattern hydrograph for the 2-year and the 10-year water levels based upon events that had peak 
water elevation values close to those in Table C-4. As discussed previously, the actual storm used will be the 11 January 1982 event.

Three storms that had a water level-return period near 2 years were 13 November 2003, 7 October 2009, and 25 December 2014. Three storms that had a wave height-return period near 10-years were 1 December 2006, 10 March 2002, and 12 December 2000. The water level hydrographs for the 2year and the 10-year synthetic events were created by plotting the three storms, adjusting the timing so all peaks align at same time, averaging the three storm water levels, and then finally adjusting all water levels based on ratio of average water elevation at peak and the determined water elevation for the 2-year or 10-year event. Figures $\mathrm{C}-11$ and $\mathrm{C}-12$ present the three storms used to create the pattern hydrographs for the 2-year and 10-year water level, respectively. Figures $\mathrm{C}-13$ to $\mathrm{C}-15$ present the water level graphs for the 2-year wave and 2-year water level event, the 20-year wave and 10year water level event, and the 11 January 1982 storm, respectively.

Figure C-11. Three storms used to create the 2-year water level hydrograph.

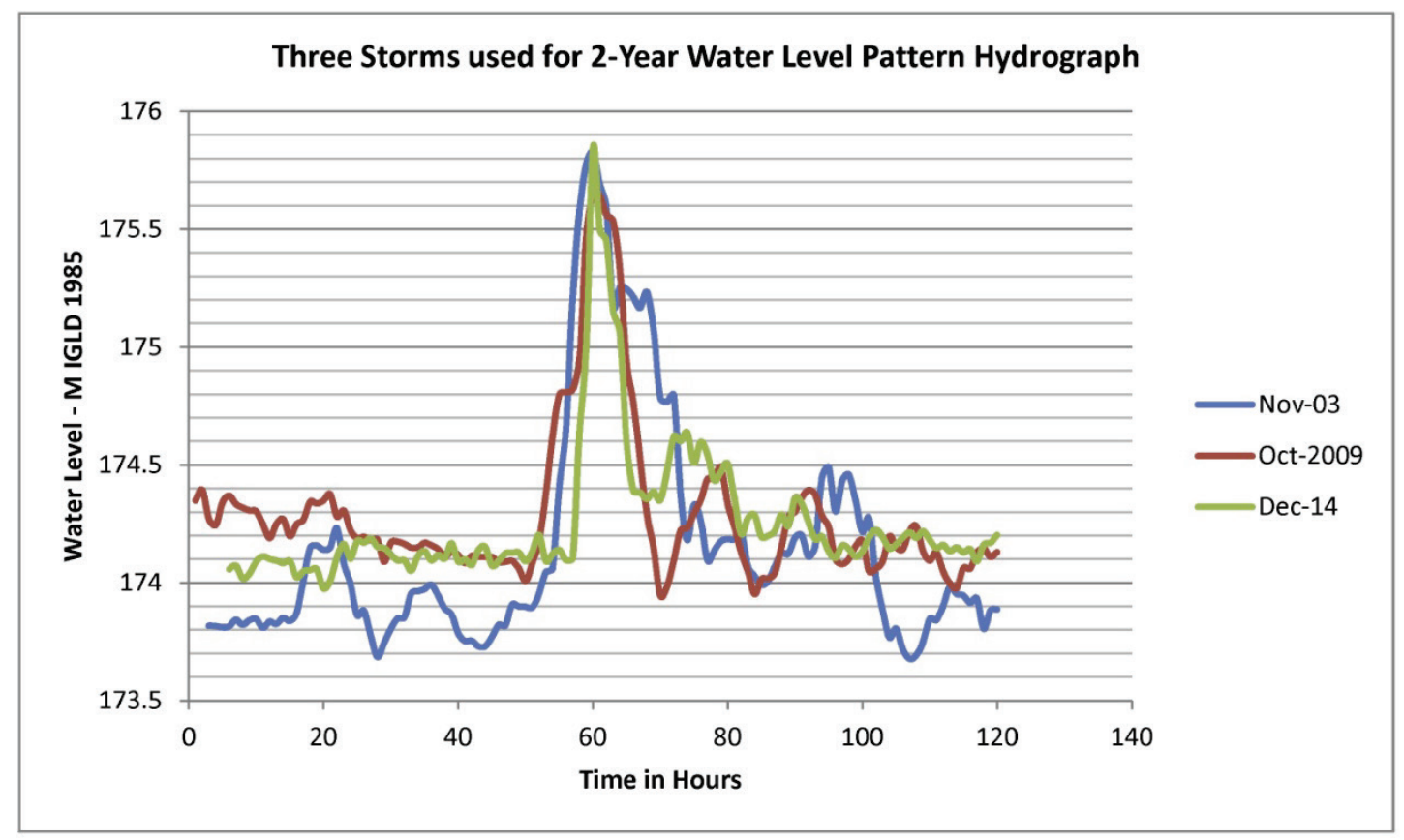


Figure C-12. Three storms used to create the 10-year water level hydrograph.

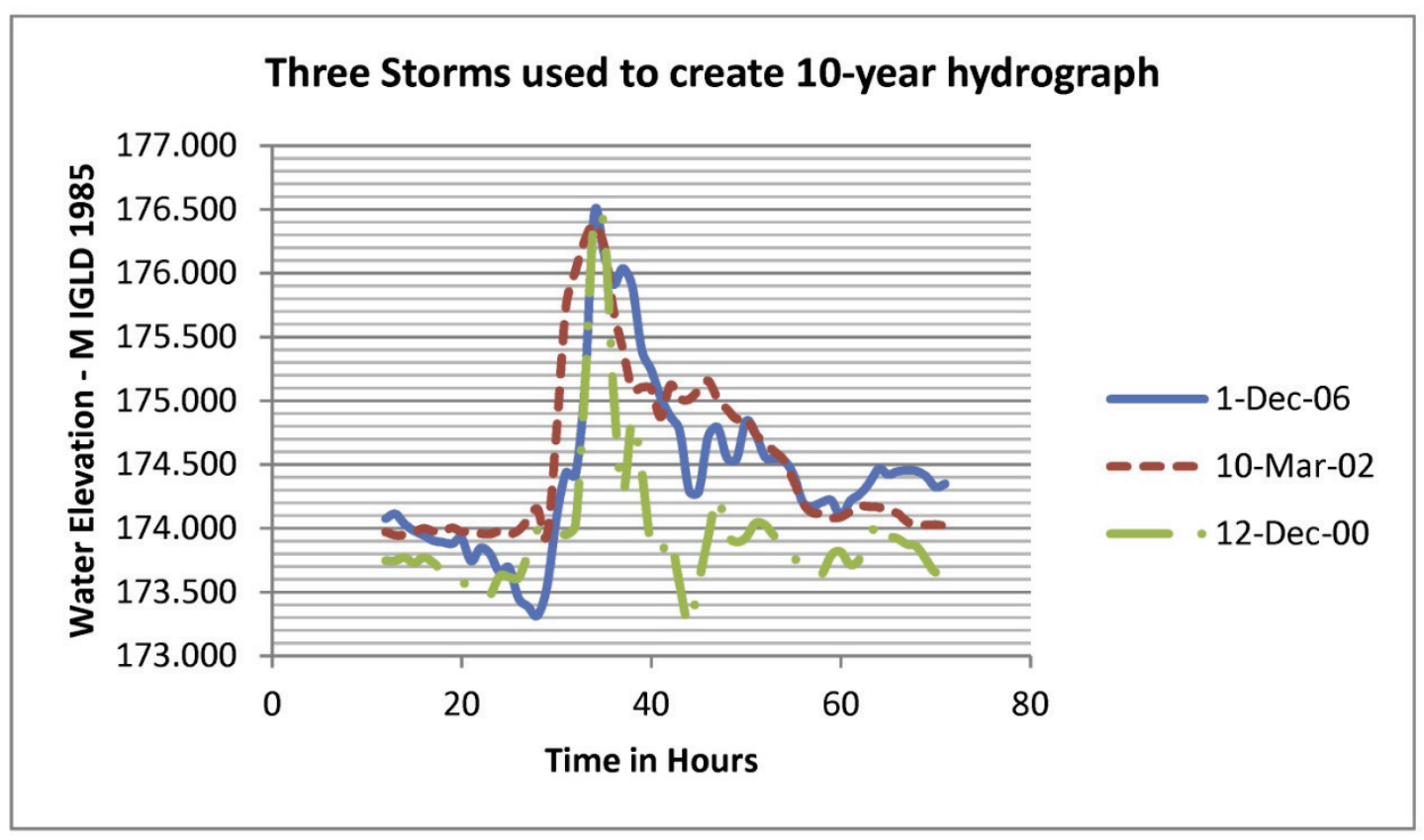

In the creation of the design storm hydrographs, it was assumed that the peak of the waves occurs coincident with the peak of the water level.

\section{C.2.7 Storm summary}

Three storms will be modeled representing (a) 2-year wave and 2-year water level, (b) 20-year wave and 10-year water level, and (c) the 11 January 2008 event. Tables C-5, C-6, and C-7 and Figures C-13, C-14, and C-15 provide hourly data of wave height, wave period, wave direction, wind speed, wind direction and water level for each storm, respectively.

Table C-5. Hourly data for 2-year wave and 2-year water level storm.

\begin{tabular}{|c|c|c|c|c|c|c|}
\hline Time - Hours & $\mathrm{H}_{\mathrm{mo}}-\mathrm{M}$ & $\mathrm{Tp}-\mathrm{Sec}$ & $\begin{array}{c}\text { Wave } \\
\text { Direction } \\
\text { Degrees } \\
\text { Azimuth }\end{array}$ & $\begin{array}{l}\text { Wind } \\
\text { Speed - } \\
\text { m/s }\end{array}$ & $\begin{array}{l}\text { Wind } \\
\text { Direction } \\
\text { Degrees } \\
\text { Azimuth }\end{array}$ & $\begin{array}{c}\text { Water Level } \\
\text { M IGLD } \\
1985\end{array}$ \\
\hline 0 & 0.55 & 3.73 & 247 & 4.4 & 247 & 174.02 \\
\hline 1 & 0.57 & 3.82 & 247 & 4.4 & 247 & 174.03 \\
\hline 2 & 0.64 & 4.01 & 247 & 4.8 & 247 & 174.06 \\
\hline 3 & 0.86 & 4.44 & 247 & 5.7 & 247 & 174.05 \\
\hline 4 & 1.10 & 4.99 & 247 & 6.8 & 247 & 174.02 \\
\hline 5 & 1.34 & 5.58 & 247 & 7.8 & 247 & 174.06 \\
\hline 6 & 1.61 & 6.16 & 247 & 8.9 & 247 & 174.13 \\
\hline
\end{tabular}




\begin{tabular}{|c|c|c|c|c|c|c|}
\hline Time - Hours & $\mathrm{H}_{\mathrm{mo}}-\mathrm{M}$ & $T_{p}-\operatorname{Sec}$ & $\begin{array}{c}\text { Wave } \\
\text { Direction } \\
\text { Degrees } \\
\text { Azimuth }\end{array}$ & $\begin{array}{l}\text { Wind } \\
\text { Speed - } \\
\text { m/s }\end{array}$ & $\begin{array}{l}\text { Wind } \\
\text { Direction } \\
\text { Degrees } \\
\text { Azimuth }\end{array}$ & $\begin{array}{c}\text { Water Level } \\
\text { M IGLD } \\
1985\end{array}$ \\
\hline 7 & 1.78 & 6.70 & 247 & 9.6 & 247 & 174.19 \\
\hline 8 & 2.04 & 6.15 & 247 & 10.6 & 247 & 174.29 \\
\hline 9 & 2.37 & 6.81 & 247 & 11.9 & 247 & 174.47 \\
\hline 10 & 2.73 & 7.43 & 247 & 13.3 & 247 & 174.54 \\
\hline 11 & 3.17 & 8.02 & 247 & 14.9 & 247 & 174.73 \\
\hline 12 & 3.63 & 8.74 & 247 & 16.5 & 247 & 175.07 \\
\hline 13 & 4.06 & 9.36 & 247 & 17.9 & 247 & 175.44 \\
\hline 14 & 4.20 & 9.80 & 247 & 18.4 & 247 & 175.80 \\
\hline 15 & 4.14 & 9.96 & 247 & 18.2 & 247 & 175.63 \\
\hline 16 & 3.87 & 9.93 & 247 & 17.3 & 247 & 175.55 \\
\hline 17 & 3.45 & 9.77 & 247 & 15.9 & 247 & 175.31 \\
\hline 18 & 3.15 & 9.32 & 247 & 14.8 & 247 & 175.23 \\
\hline 19 & 2.99 & 9.04 & 247 & 14.2 & 247 & 174.95 \\
\hline 20 & 2.76 & 8.52 & 247 & 13.4 & 247 & 174.81 \\
\hline 21 & 2.44 & 8.27 & 247 & 12.2 & 247 & 174.71 \\
\hline 22 & 2.15 & 7.77 & 247 & 11.1 & 247 & 174.65 \\
\hline 23 & 1.88 & 7.49 & 247 & 10.0 & 247 & 174.56 \\
\hline 24 & 1.69 & 7.09 & 247 & 9.2 & 247 & 174.38 \\
\hline 25 & 1.54 & 6.82 & 247 & 8.6 & 247 & 174.43 \\
\hline 26 & 1.38 & 6.71 & 247 & 7.9 & 247 & 174.52 \\
\hline 27 & 1.23 & 6.32 & 247 & 7.3 & 247 & 174.42 \\
\hline 28 & 1.08 & 6.21 & 247 & 6.7 & 247 & 174.37 \\
\hline 29 & 0.98 & 6.26 & 247 & 6.2 & 247 & 174.40 \\
\hline 30 & 0.93 & 5.49 & 247 & 6.0 & 247 & 174.42 \\
\hline 31 & 0.85 & 5.48 & 247 & 5.7 & 247 & 174.38 \\
\hline 32 & 0.78 & 5.25 & 247 & 5.4 & 247 & 174.36 \\
\hline 33 & 0.72 & 5.25 & 247 & 5.1 & 247 & 174.40 \\
\hline 34 & 0.70 & 5.09 & 247 & 5.0 & 247 & 174.36 \\
\hline 35 & 0.71 & 5.11 & 247 & 5.1 & 247 & 174.28 \\
\hline 36 & 0.70 & 5.23 & 247 & 5.0 & 247 & 174.20 \\
\hline 37 & 0.65 & 5.26 & 247 & 4.8 & 247 & 174.15 \\
\hline
\end{tabular}




\begin{tabular}{|c|c|c|c|c|c|c|}
\hline Time - Hours & Hmo $-M$ & $\mathrm{TP}_{\mathrm{P}}$-Sec & $\begin{array}{c}\text { Wave } \\
\text { Direction } \\
\text { Degrees } \\
\text { Azimuth }\end{array}$ & $\begin{array}{c}\text { Wind } \\
\text { Speed - } \\
\text { m/s }\end{array}$ & $\begin{array}{c}\text { Wind } \\
\text { Direction } \\
\text { Degrees } \\
\text { Azimuth }\end{array}$ & $\begin{array}{c}\text { Water Level } \\
\text { M IGLD } \\
1985\end{array}$ \\
\hline 38 & 0.61 & 5.27 & 247 & 4.6 & 247 & 174.11 \\
\hline 39 & 0.57 & 5.19 & 247 & 4.4 & 247 & 174.09 \\
\hline 40 & 0.54 & 5.05 & 247 & 4.3 & 247 & 174.10 \\
\hline 41 & 0.52 & 4.97 & 247 & 4.2 & 247 & 174.13 \\
\hline 42 & 0.50 & 4.94 & 247 & 4.1 & 247 & 174.21 \\
\hline 43 & 0.47 & 4.88 & 247 & 4.0 & 247 & 174.23 \\
\hline 44 & 0.45 & 4.22 & 247 & 3.9 & 247 & 174.30 \\
\hline 45 & 0.43 & 4.33 & 247 & 3.8 & 247 & 174.32 \\
\hline 46 & 0.42 & 4.47 & 247 & 3.8 & 247 & 174.27 \\
\hline 47 & 0.42 & 4.52 & 247 & 3.8 & 247 & 174.26 \\
\hline 48 & 0.45 & 3.90 & 247 & 3.9 & 247 & 174.33 \\
\hline 49 & 0.47 & 4.10 & 247 & 4.0 & 247 & 174.31 \\
\hline 50 & 0.50 & 4.29 & 247 & 4.1 & 247 & 174.19 \\
\hline & & & & & & \\
\hline
\end{tabular}


Figure C-13. Time series data for the 2-year wave and 2-year water level storm.

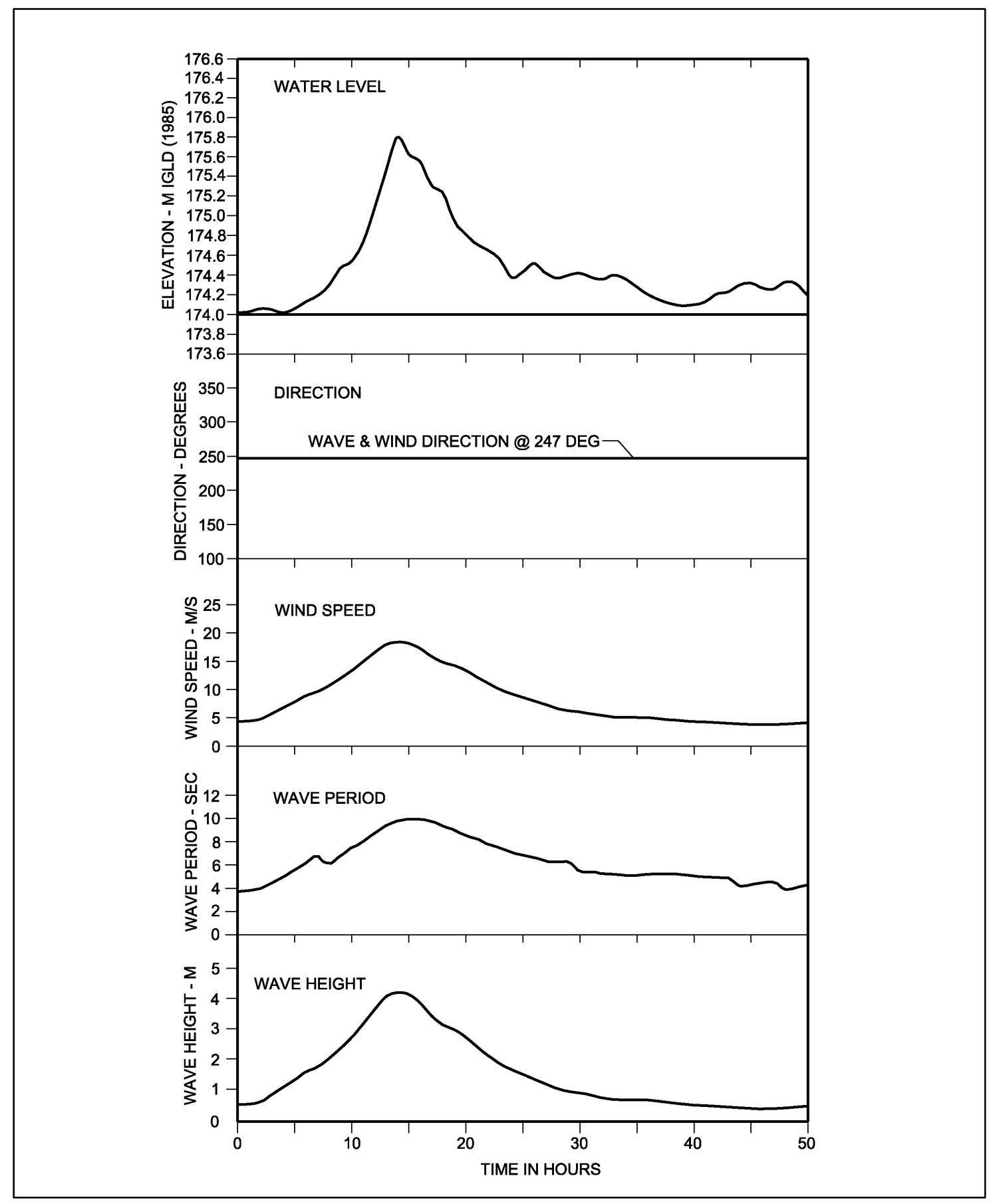


Table C-6. Hourly data for 20-year wave and 10-year water level storm.

\begin{tabular}{|c|c|c|c|c|c|c|}
\hline Time - Hours & $\mathrm{H}_{\mathrm{mo}}-\mathrm{M}$ & $\mathrm{TP}-\mathrm{Sec}$ & $\begin{array}{c}\text { Wave } \\
\text { Direction } \\
\text { Degrees } \\
\text { Azimuth }\end{array}$ & $\begin{array}{l}\text { Wind } \\
\text { Speed - } \\
\text { m/s }\end{array}$ & $\begin{array}{c}\text { Wind } \\
\text { Direction } \\
\text { Degrees } \\
\text { Azimuth }\end{array}$ & $\begin{array}{c}\text { Water Level } \\
\text { M IGLD } \\
1985\end{array}$ \\
\hline 0 & 0.34 & 2.94 & 247 & 3.4 & 247 & 173.84 \\
\hline 1 & 0.34 & 3.10 & 247 & 3.4 & 247 & 173.77 \\
\hline 2 & 0.34 & 3.13 & 247 & 3.4 & 247 & 173.77 \\
\hline 3 & 0.34 & 3.17 & 247 & 3.4 & 247 & 173.75 \\
\hline 4 & 0.36 & 3.23 & 247 & 3.5 & 247 & 173.77 \\
\hline 5 & 0.40 & 2.91 & 247 & 3.7 & 247 & 173.77 \\
\hline 6 & 0.50 & 3.21 & 247 & 4.1 & 247 & 173.69 \\
\hline 7 & 0.62 & 3.43 & 247 & 4.7 & 247 & 173.76 \\
\hline 8 & 0.79 & 3.89 & 247 & 5.4 & 247 & 173.83 \\
\hline 9 & 1.36 & 5.12 & 247 & 7.9 & 247 & 173.85 \\
\hline 10 & 2.16 & 6.20 & 247 & 11.1 & 247 & 174.29 \\
\hline 11 & 2.95 & 7.22 & 247 & 14.1 & 247 & 174.72 \\
\hline 12 & 3.77 & 8.48 & 247 & 17.0 & 247 & 174.83 \\
\hline 13 & 4.45 & 9.63 & 247 & 19.2 & 247 & 175.51 \\
\hline 14 & 4.80 & 10.40 & 247 & 20.2 & 247 & 176.45 \\
\hline 15 & 4.54 & 10.63 & 247 & 19.4 & 247 & 176.27 \\
\hline 16 & 3.96 & 10.45 & 247 & 17.6 & 247 & 175.58 \\
\hline 17 & 3.50 & 10.10 & 247 & 16.0 & 247 & 175.25 \\
\hline 18 & 3.17 & 9.72 & 247 & 14.9 & 247 & 175.29 \\
\hline 19 & 2.91 & 9.30 & 247 & 13.9 & 247 & 174.99 \\
\hline 20 & 2.77 & 9.06 & 247 & 13.4 & 247 & 174.74 \\
\hline 21 & 2.63 & 8.73 & 247 & 12.9 & 247 & 174.58 \\
\hline 22 & 2.41 & 8.51 & 247 & 12.1 & 247 & 174.65 \\
\hline 23 & 2.16 & 8.16 & 247 & 11.1 & 247 & 174.46 \\
\hline 24 & 1.94 & 7.94 & 247 & 10.2 & 247 & 174.19 \\
\hline 25 & 1.74 & 7.66 & 247 & 9.4 & 247 & 174.33 \\
\hline 26 & 1.61 & 7.47 & 247 & 8.9 & 247 & 174.61 \\
\hline 27 & 1.54 & 7.33 & 247 & 8.6 & 247 & 174.70 \\
\hline 28 & 1.47 & 7.23 & 247 & 8.3 & 247 & 174.49 \\
\hline 29 & 1.37 & 7.22 & 247 & 7.9 & 247 & 174.44 \\
\hline 30 & 1.24 & 7.23 & 247 & 7.4 & 247 & 174.56 \\
\hline
\end{tabular}




\begin{tabular}{|c|c|c|c|c|c|c|}
\hline Time - Hours & $\mathrm{H}_{\mathrm{mo}}-\mathrm{M}$ & $T_{P}-\operatorname{Sec}$ & $\begin{array}{l}\text { Wave } \\
\text { Direction } \\
\text { Degrees } \\
\text { Azimuth }\end{array}$ & $\begin{array}{l}\text { Wind } \\
\text { Speed - } \\
\mathrm{m} / \mathrm{s}\end{array}$ & $\begin{array}{l}\text { Wind } \\
\text { Direction } \\
\text { Degrees } \\
\text { Azimuth }\end{array}$ & $\begin{array}{c}\text { Water Level } \\
\text { M IGLD } \\
1985\end{array}$ \\
\hline 31 & 1.12 & 6.84 & 247 & 6.8 & 247 & 174.52 \\
\hline 32 & 1.03 & 6.75 & 247 & 6.5 & 247 & 174.43 \\
\hline 33 & 0.97 & 6.83 & 247 & 6.2 & 247 & 174.37 \\
\hline 34 & 0.92 & 6.68 & 247 & 6.0 & 247 & 174.33 \\
\hline 35 & 0.87 & 6.62 & 247 & 5.8 & 247 & 174.20 \\
\hline 36 & 0.83 & 6.45 & 247 & 5.6 & 247 & 174.04 \\
\hline 37 & 0.80 & 6.11 & 247 & 5.5 & 247 & 173.99 \\
\hline 38 & 0.77 & 6.00 & 247 & 5.3 & 247 & 174.00 \\
\hline 39 & 0.72 & 6.04 & 247 & 5.1 & 247 & 174.04 \\
\hline 40 & 0.69 & 5.97 & 247 & 5.0 & 247 & 174.01 \\
\hline 41 & 0.66 & 4.86 & 247 & 4.8 & 247 & 174.03 \\
\hline 42 & 0.65 & 4.81 & 247 & 4.8 & 247 & 174.08 \\
\hline 43 & 0.64 & 4.79 & 247 & 4.8 & 247 & 174.16 \\
\hline 44 & 0.62 & 4.75 & 247 & 4.7 & 247 & 174.24 \\
\hline 45 & 0.60 & 4.70 & 247 & 4.6 & 247 & 174.18 \\
\hline 46 & 0.61 & 4.60 & 247 & 4.6 & 247 & 174.17 \\
\hline 47 & 0.62 & 4.66 & 247 & 4.7 & 247 & 174.14 \\
\hline 48 & 0.65 & 4.80 & 247 & 4.8 & 247 & 174.12 \\
\hline 49 & 0.71 & 5.00 & 247 & 5.1 & 247 & 174.07 \\
\hline 50 & 0.80 & 5.01 & 247 & 5.5 & 247 & 174.01 \\
\hline
\end{tabular}


Figure C-14. Time series data for the 20-year wave and 10-year water level storm.

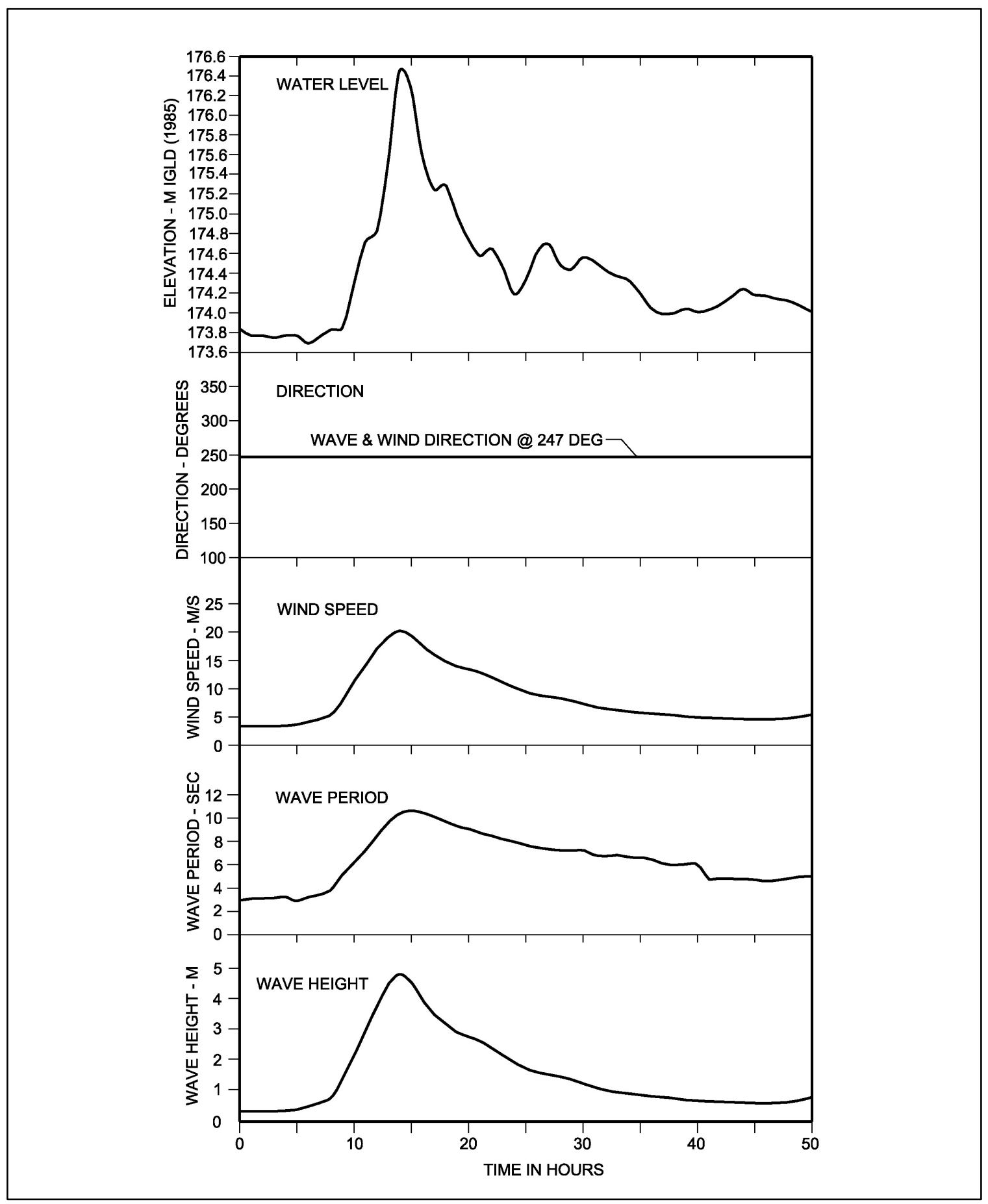

Table C-7. Hourly data for the 11 January 1982 storm.

\begin{tabular}{|c|c|c|c|c|c|c|}
\hline & Date and Time & $\mathrm{H}_{\mathrm{mo}}-\mathrm{M}$ & $\begin{array}{c}\text { Wave } \\
\text { Direction } \\
\text { Degrees } \\
\text { Azimuth }\end{array}$ & $\begin{array}{c}\text { Wind } \\
\text { Speed - } \\
\mathrm{m} / \mathrm{s}\end{array}$ & $\begin{array}{c}\text { Wind } \\
\text { Direction } \\
\text { Degrees } \\
\text { Azimuth }\end{array}$ & $\begin{array}{c}\text { Water Level } \\
\text { M IGLD } \\
1985\end{array}$ \\
\hline $1 / 10 / 198215: 00$ & 1.06 & 3.90 & 260 & 11.6 & 286 & 174.637 \\
\hline
\end{tabular}




\begin{tabular}{|c|c|c|c|c|c|c|}
\hline Date and Time & $\mathrm{H}_{\mathrm{mo}}-\mathrm{M}$ & $T_{p}-\operatorname{Sec}$ & $\begin{array}{c}\text { Wave } \\
\text { Direction } \\
\text { Degrees } \\
\text { Azimuth }\end{array}$ & $\begin{array}{l}\text { Wind } \\
\text { Speed - } \\
\text { m/s }\end{array}$ & $\begin{array}{c}\text { Wind } \\
\text { Direction } \\
\text { Degrees } \\
\text { Azimuth }\end{array}$ & $\begin{array}{c}\text { Water Level } \\
\text { M IGLD } \\
1985\end{array}$ \\
\hline 1/10/1982 16:00 & 1.47 & 4.94 & 260 & 12.4 & 276 & 174.917 \\
\hline 1/10/1982 17:00 & 1.93 & 5.82 & 257 & 13.4 & 270 & 175.119 \\
\hline 1/10/1982 18:00 & 2.52 & 6.66 & 254 & 15.2 & 262 & 175.116 \\
\hline 1/10/1982 19:00 & 2.97 & 7.46 & 252 & 15.8 & 261 & 175.027 \\
\hline 1/10/1982 20:00 & 3.19 & 8.24 & 252 & 15.8 & 260 & 175.609 \\
\hline 1/10/1982 21:00 & 3.24 & 8.82 & 251 & 15.4 & 258 & 175.981 \\
\hline 1/10/1982 22:00 & 3.34 & 9.15 & 249 & 15.9 & 253 & 176.149 \\
\hline 1/10/1982 23:00 & 3.56 & 9.59 & 249 & 16.7 & 249 & 176.191 \\
\hline 1/11/1982 0:00 & 3.86 & 9.98 & 247 & 19.8 & 233 & 176.362 \\
\hline 1/11/1982 1:00 & 4.28 & 10.20 & 248 & 20.3 & 243 & 176.176 \\
\hline 1/11/1982 2:00 & 4.34 & 10.46 & 248 & 19.2 & 244 & 176.048 \\
\hline 1/11/1982 3:00 & 4.12 & 10.67 & 248 & 18.4 & 243 & 175.844 \\
\hline 1/11/1982 4:00 & 3.93 & 10.65 & 247 & 17.5 & 242 & 175.804 \\
\hline 1/11/1982 5:00 & 3.69 & 10.44 & 247 & 16.5 & 241 & 175.652 \\
\hline 1/11/1982 6:00 & 3.47 & 10.20 & 247 & 15.6 & 243 & 175.567 \\
\hline 1/11/1982 7:00 & 3.35 & 10.00 & 247 & 16.1 & 242 & 175.466 \\
\hline 1/11/1982 8:00 & 3.37 & 9.84 & 247 & 16.7 & 241 & 175.582 \\
\hline 1/11/1982 9:00 & 3.52 & 9.76 & 248 & 17.8 & 240 & 175.707 \\
\hline 1/11/1982 10:00 & 3.82 & 9.80 & 248 & 19.4 & 240 & 175.801 \\
\hline 1/11/1982 11:00 & 4.20 & 10.00 & 248 & 20.4 & 240 & 175.820 \\
\hline 1/11/1982 12:00 & 4.37 & 10.17 & 247 & 21.2 & 229 & 175.978 \\
\hline 1/11/1982 13:00 & 4.25 & 10.30 & 247 & 18.7 & 245 & 176.015 \\
\hline 1/11/1982 14:00 & 3.70 & 10.30 & 248 & 14.6 & 263 & 175.844 \\
\hline 1/11/1982 15:00 & 3.03 & 10.12 & 248 & 12.0 & 284 & 175.719 \\
\hline 1/11/1982 16:00 & 2.63 & 9.98 & 248 & 12.2 & 282 & 175.579 \\
\hline 1/11/1982 17:00 & 2.59 & 9.92 & 247 & 12.5 & 279 & 175.399 \\
\hline 1/11/1982 18:00 & 2.77 & 9.86 & 248 & 13.4 & 275 & 175.557 \\
\hline 1/11/1982 19:00 & 2.91 & 9.86 & 248 & 13.5 & 278 & 175.469 \\
\hline 1/11/1982 20:00 & 2.85 & 9.86 & 248 & 12.3 & 282 & 175.271 \\
\hline 1/11/1982 21:00 & 2.63 & 9.80 & 248 & 11.0 & 284 & 175.213 \\
\hline 1/11/1982 22:00 & 2.38 & 9.66 & 248 & 9.8 & 290 & 175.158 \\
\hline
\end{tabular}




\begin{tabular}{|l|c|c|c|c|c|c|}
\hline \multicolumn{1}{|c|}{ Date and Time } & $\mathrm{H}_{\mathrm{mo}}-\mathrm{M}$ & $\mathrm{TP}_{\mathrm{P}}$-Sec & $\begin{array}{c}\text { Wave } \\
\text { Direction } \\
\text { Degrees } \\
\text { Azimuth }\end{array}$ & $\begin{array}{c}\text { Wind } \\
\text { Speed - } \\
\mathrm{m} / \mathrm{s}\end{array}$ & $\begin{array}{c}\text { Wind } \\
\text { Direction } \\
\text { Degrees } \\
\text { Azimuth }\end{array}$ & $\begin{array}{c}\text { Water Level } \\
\text { M IGLD } \\
1985\end{array}$ \\
\hline 1/11/1982 23:00 & 2.15 & 9.34 & 247 & 8.7 & 297 & 174.911 \\
\hline 1/12/1982 0:00 & 1.97 & 9.16 & 247 & 7.9 & 297 & 174.814 \\
\hline $1 / 12 / 19821: 00$ & 1.81 & 9.09 & 247 & 7.1 & 316 & 174.735 \\
\hline $1 / 12 / 19822: 00$ & 1.66 & 9.04 & 246 & 7.1 & 334 & 174.597 \\
\hline $1 / 12 / 19823: 00$ & 1.51 & 8.96 & 246 & 6.7 & 349 & 174.515 \\
\hline $1 / 12 / 19824: 00$ & 1.35 & 8.70 & 246 & 5.9 & 355 & 174.515 \\
\hline $1 / 12 / 19825: 00$ & 1.21 & 8.38 & 246 & 5.5 & 3 & 174.478 \\
\hline $1 / 12 / 19826: 00$ & 1.09 & 8.28 & 246 & 4.9 & 3 & 174.411 \\
\hline $1 / 10 / 198215: 00$ & 1.06 & 3.90 & 260 & 11.6 & 286 & 174.637 \\
\hline $1 / 10 / 198216: 00$ & 1.47 & 4.94 & 260 & 12.4 & 276 & 174.917 \\
\hline $1 / 10 / 198217: 00$ & 1.93 & 5.82 & 257 & 13.4 & 270 & 175.119 \\
\hline $1 / 10 / 198218: 00$ & 2.52 & 6.66 & 254 & 15.2 & 262 & 175.116 \\
\hline
\end{tabular}


Figure C-15. Time series data for the 11 January 1982 storm.

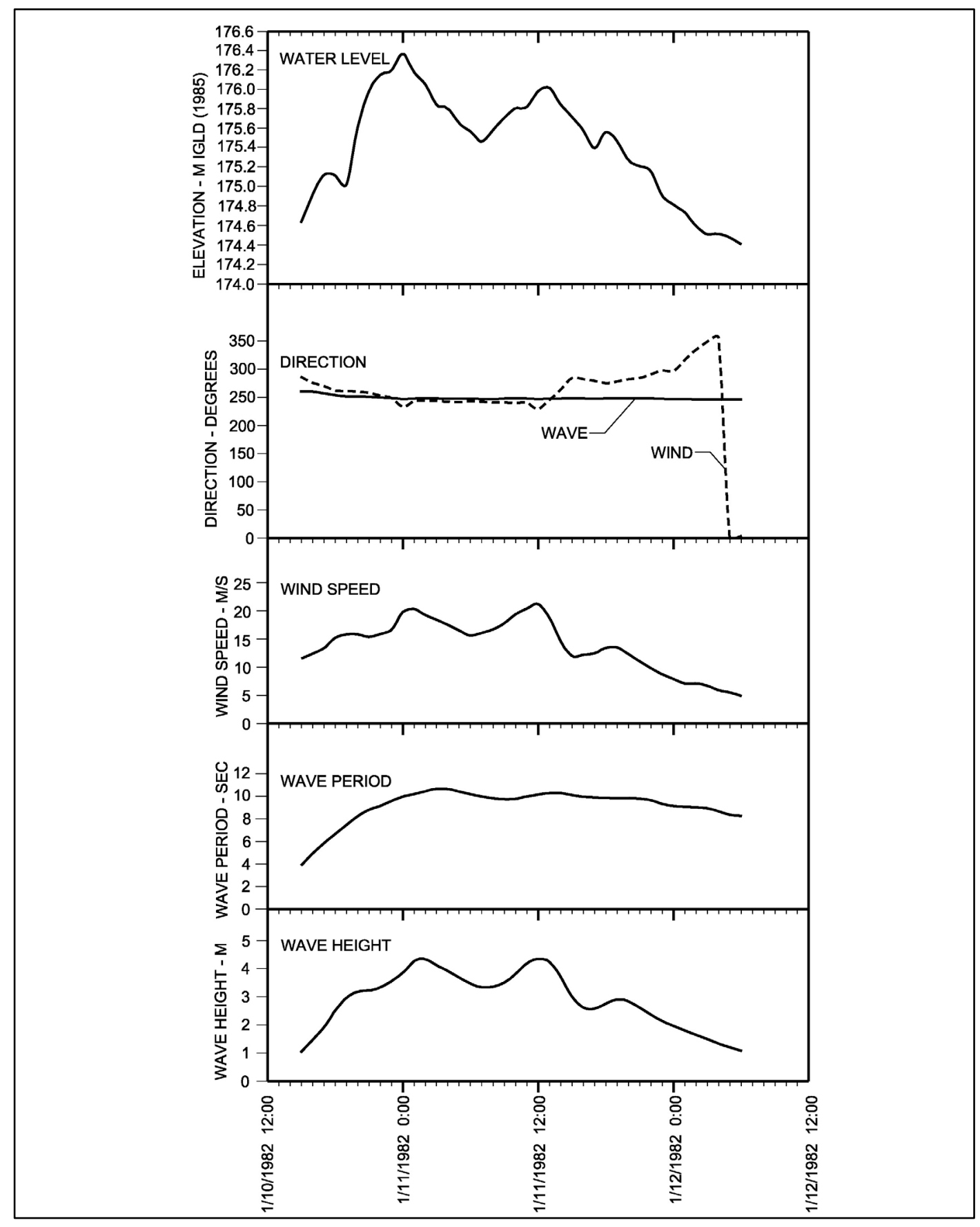




\section{Appendix D: Overtopping Estimates for Transects T1 to T10}

B2D/B1D modeling results for wave overtopping along three transects (T2, $\mathrm{T}_{5}$, and $\mathrm{T} 9$ ) were provided in Section 4.5. Results for the remaining seven transects ( $\mathrm{T}_{1}, \mathrm{~T}_{3}, \mathrm{~T}_{4}, \mathrm{~T} 6, \mathrm{~T}_{7}, \mathrm{~T} 8$, and $\left.\mathrm{T} 10\right)$ are presented here. Details of B2D/B1D modeling and analyses are provided in Chapter 4.

\section{D.1 Results for Transect T1}

Figure D-1. Variation in wave height along T1.

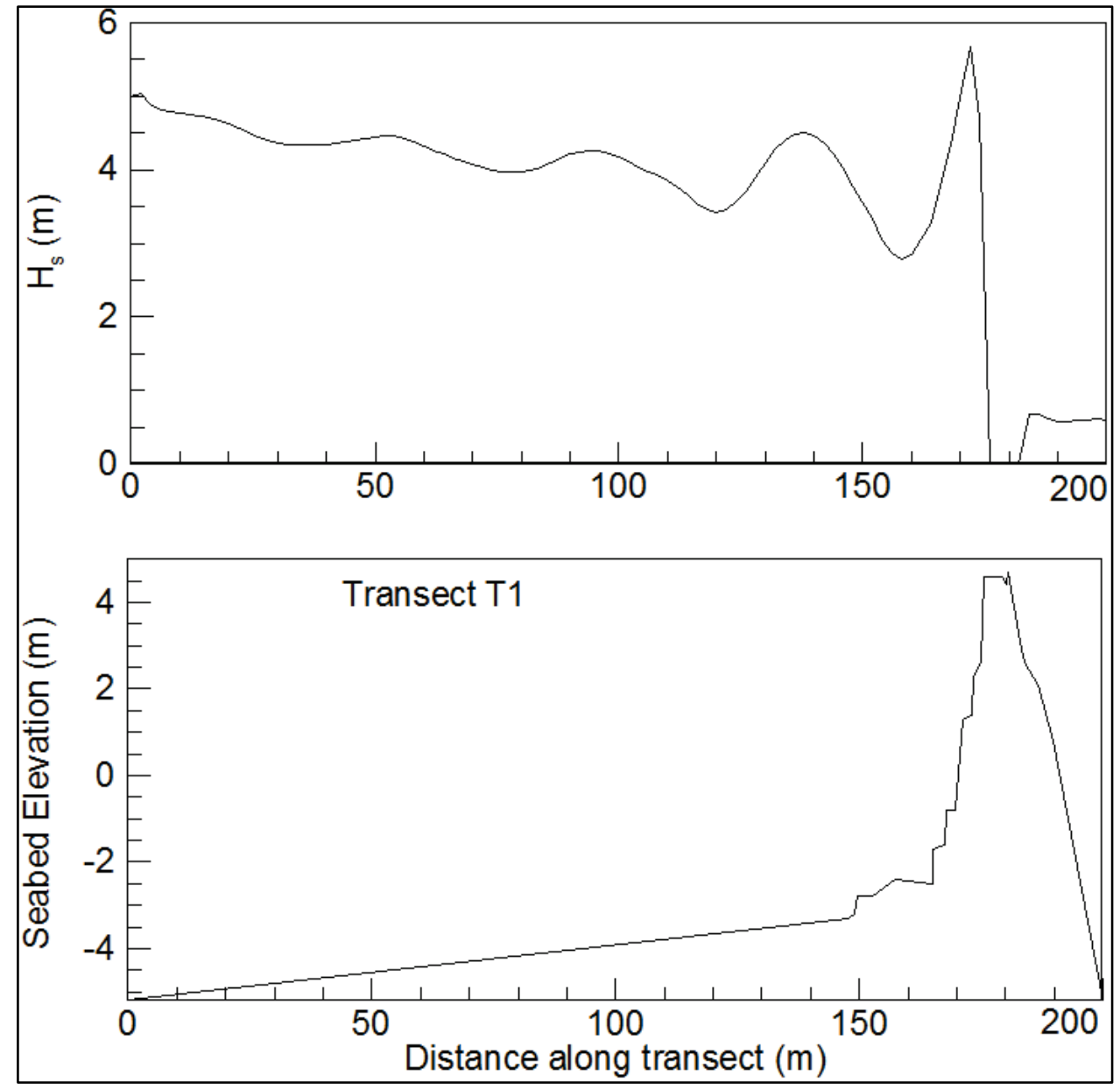


Figure D-2. Variation of overtopping rate and runup height along T1 (first $1,000 \mathrm{sec})$.

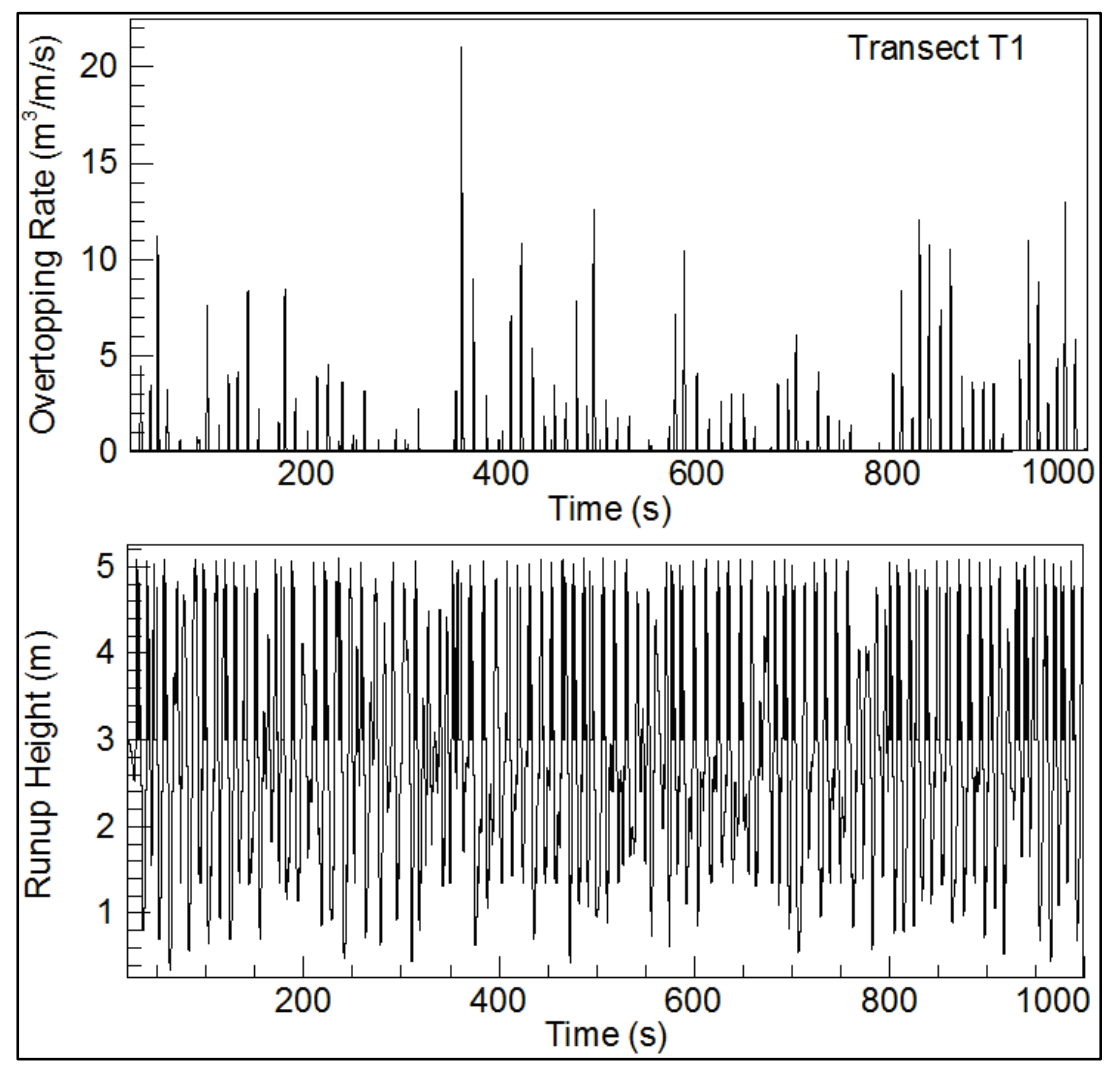

Figure D-3. Temporal variation of overtopping rate along T1 (entire simulation).

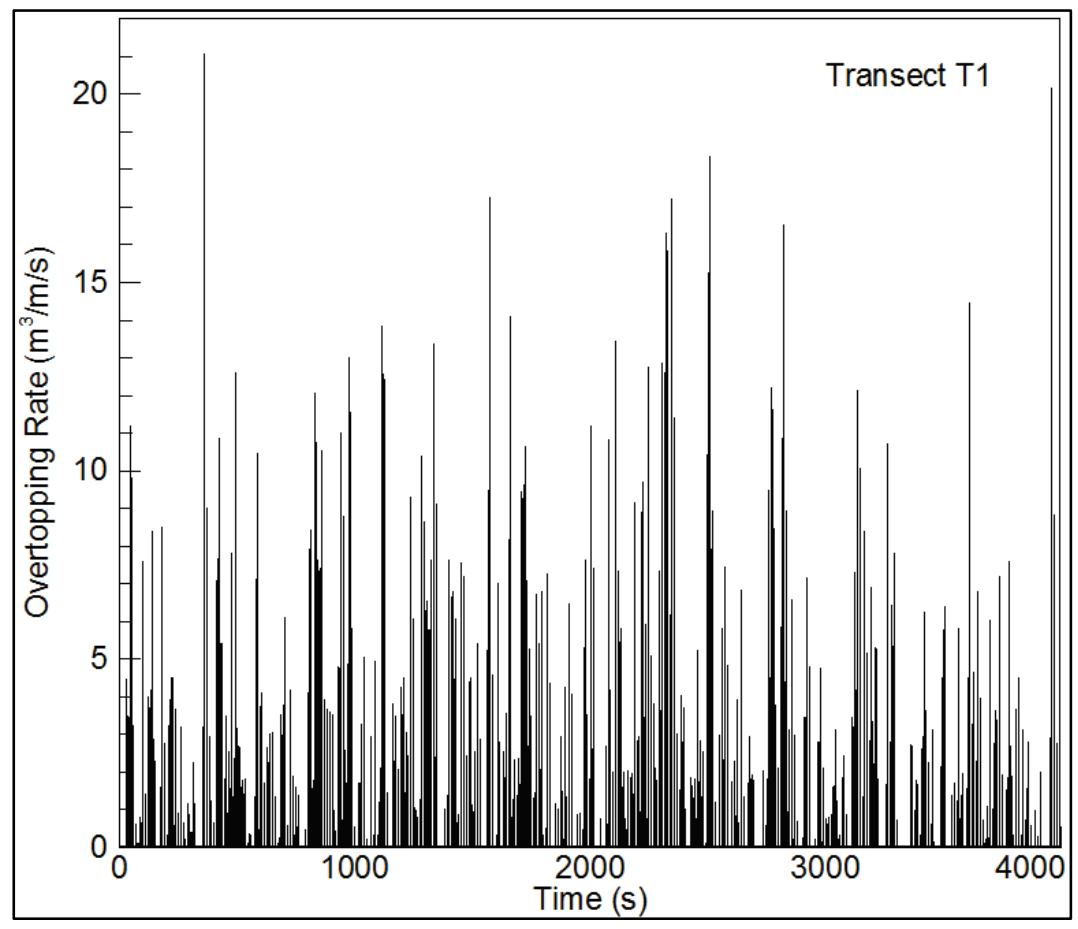




\section{D.2 Results for Transect T3}

Figure D-4. Wave height variation along T3.

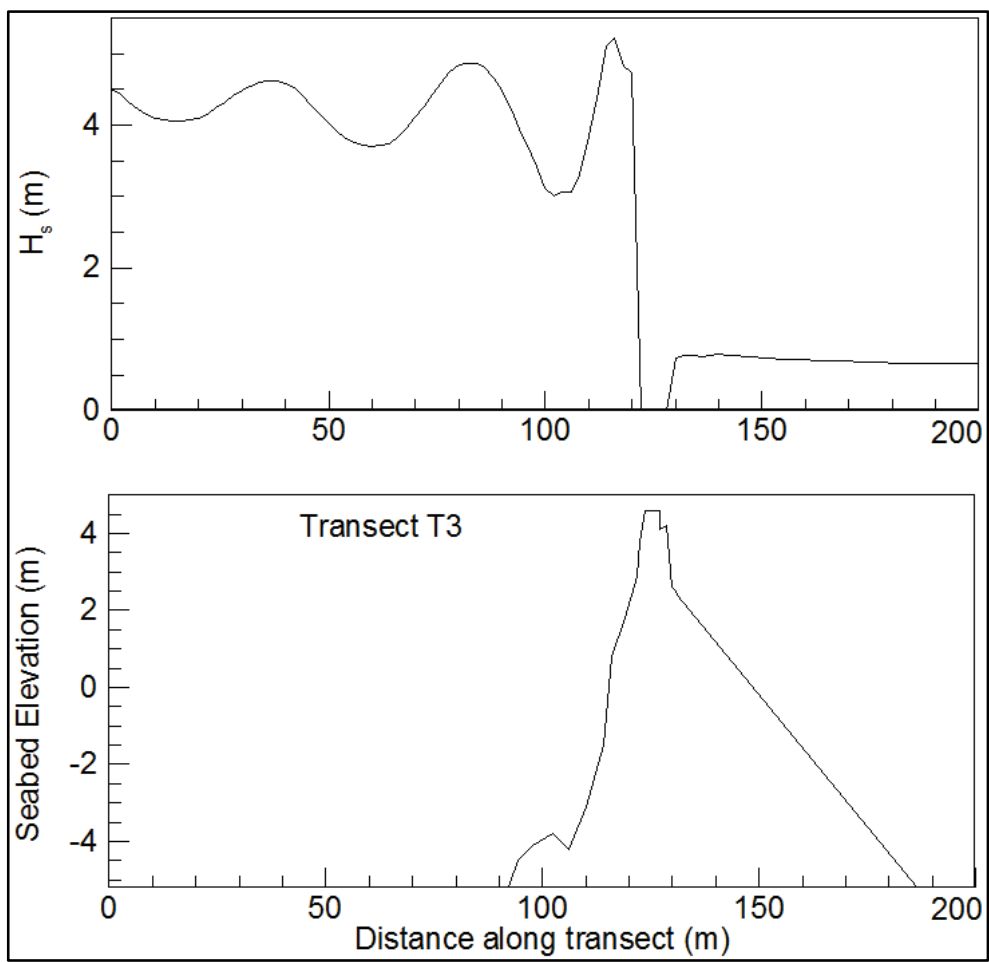

Figure D-5. Overtopping rate and runup height variation along T3 (first 1,000 sec).

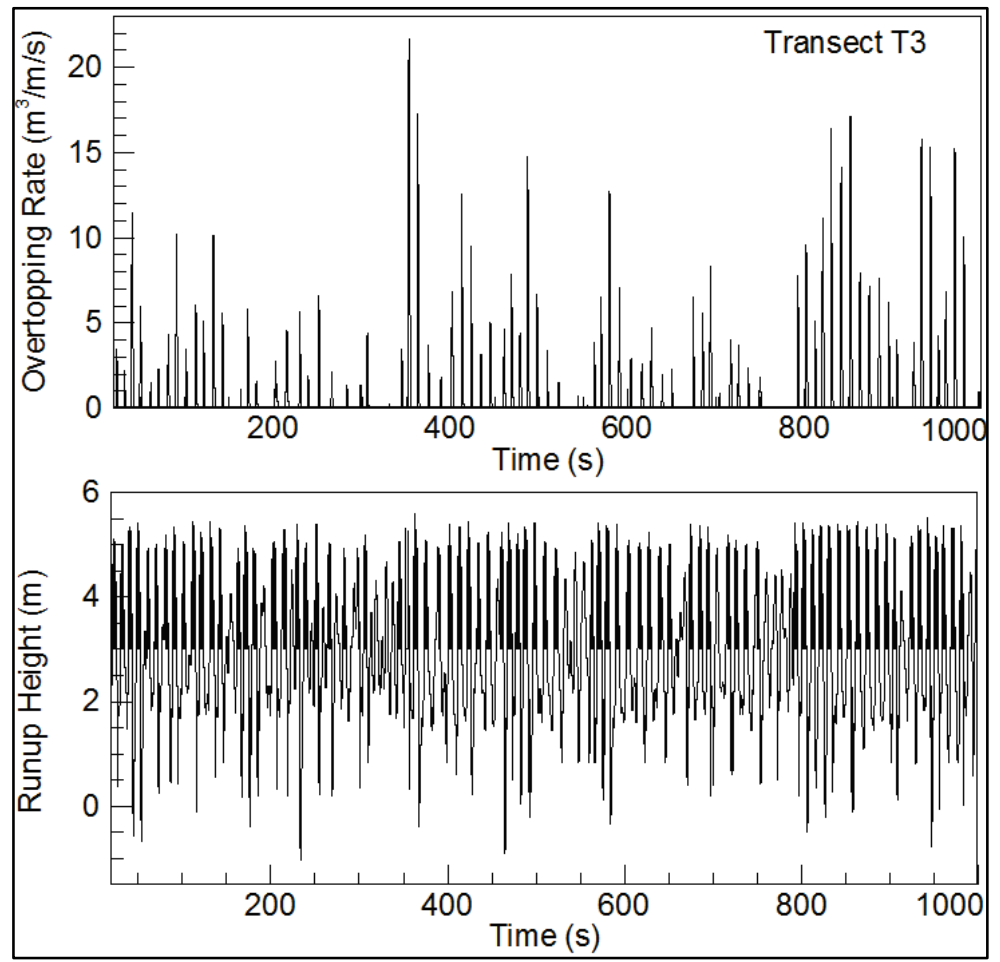


Figure D-6. Time variation of overtopping rate along T3 (entire simulation).

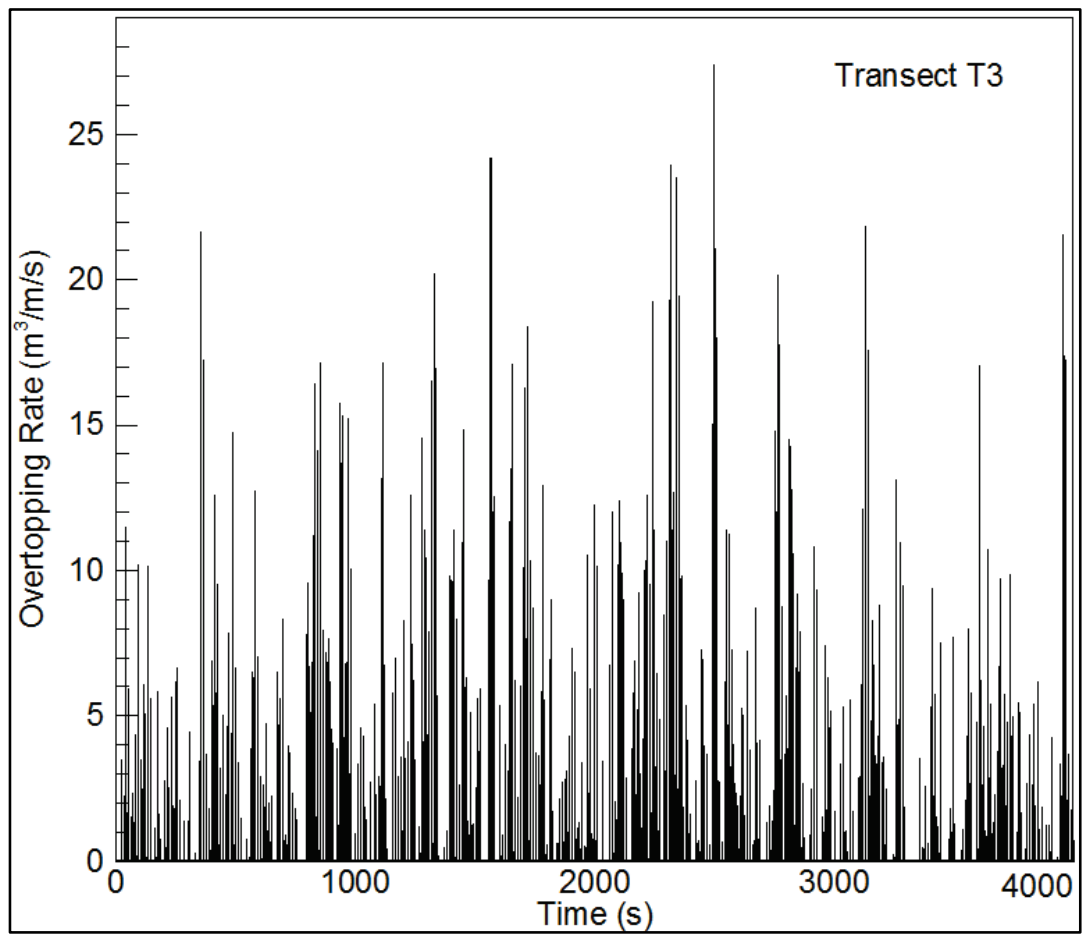




\section{D.3 Results for Transect T4}

Figure D-7. Wave height variation along T4.

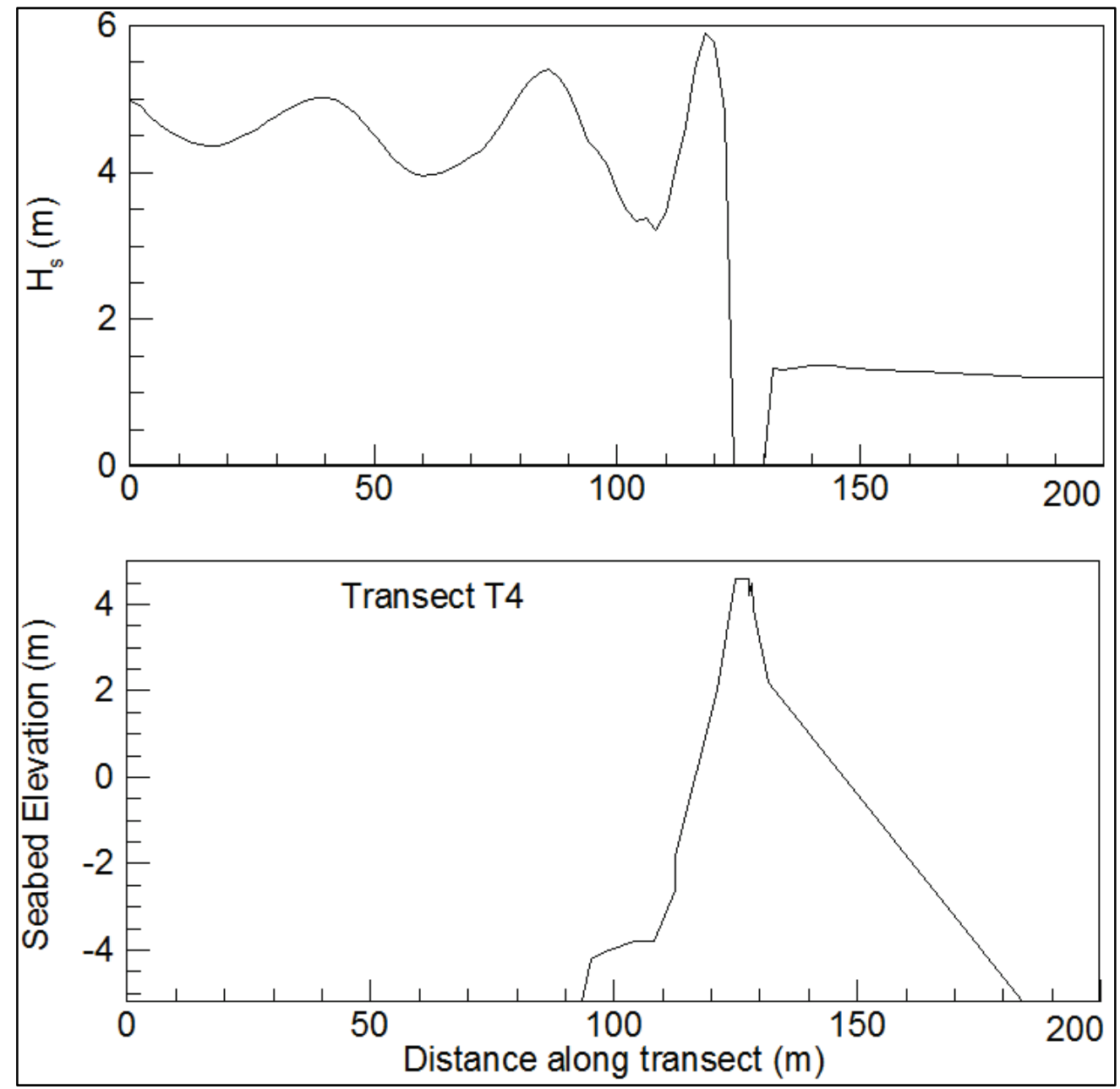


Figure D-8. Overtopping rate and runup height variation along T4 (first 1,000 sec).
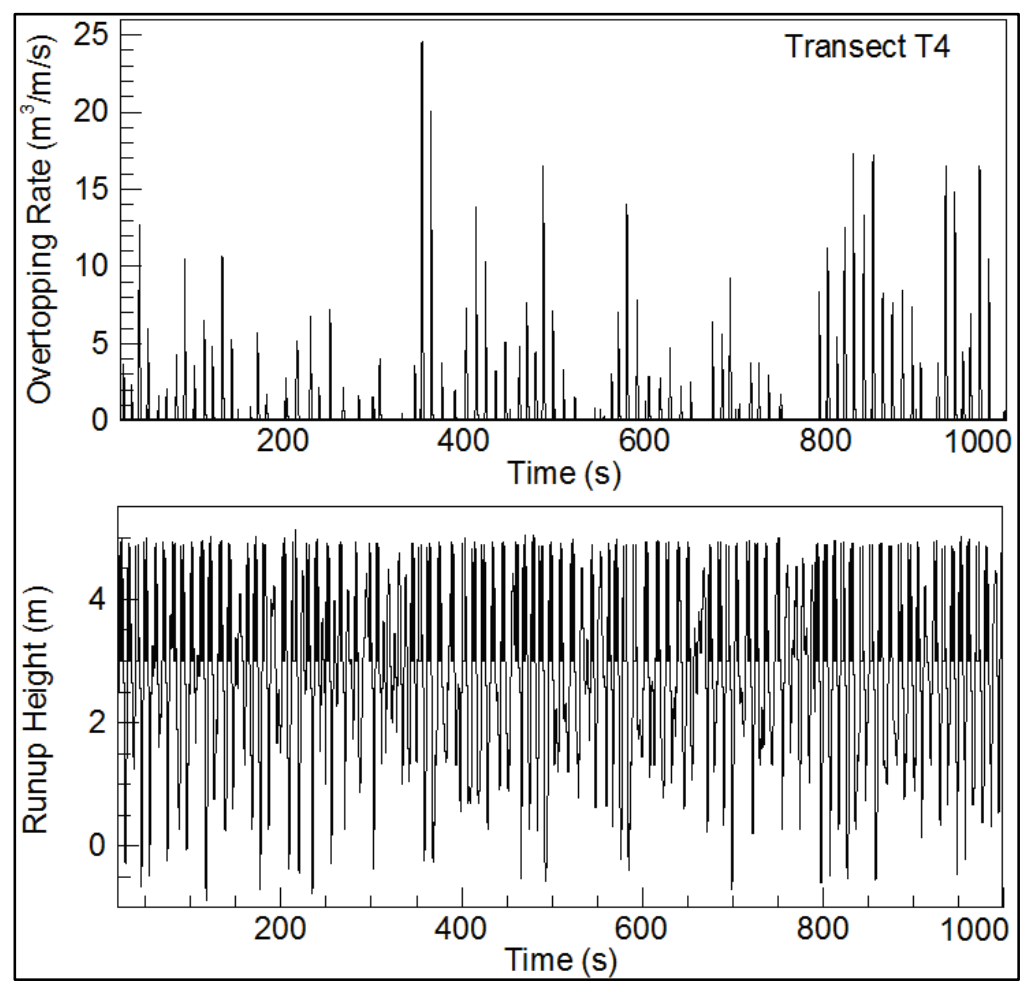

Figure D-9. Time variation of overtopping rate along T4 (entire simulation).

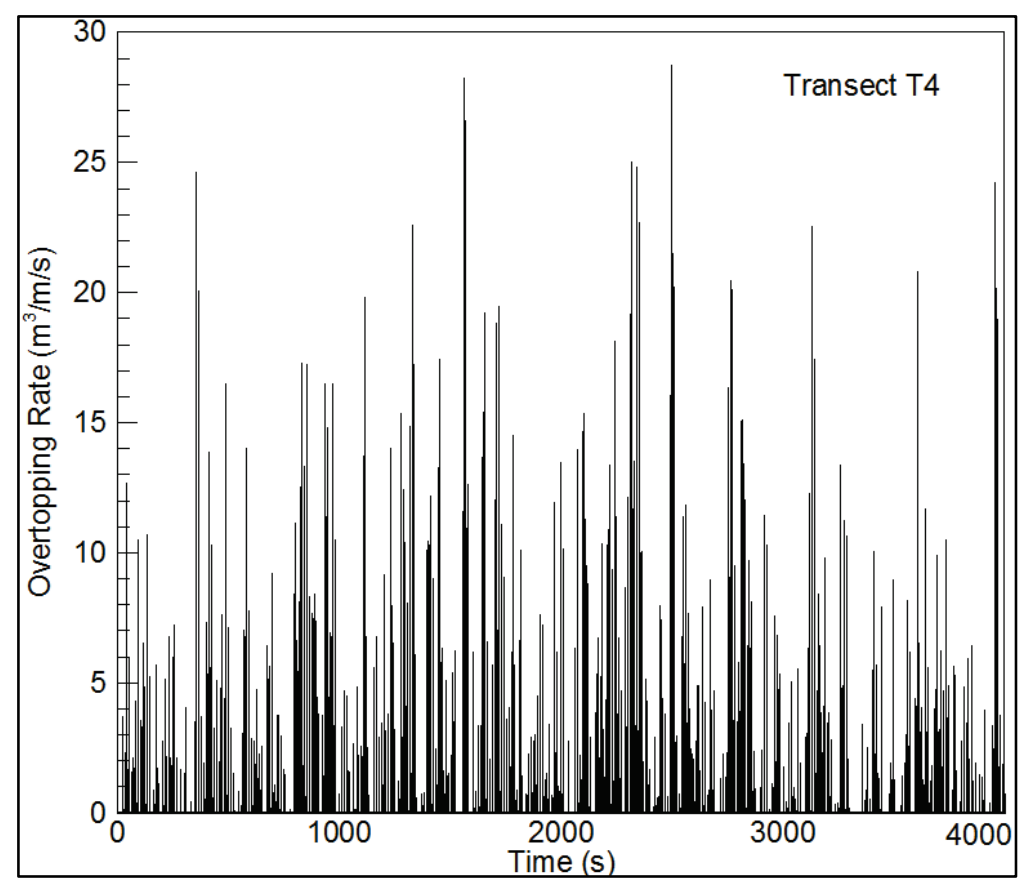




\section{D.4 Results for Transect T6}

Figure D-10. Wave height variation along T6.

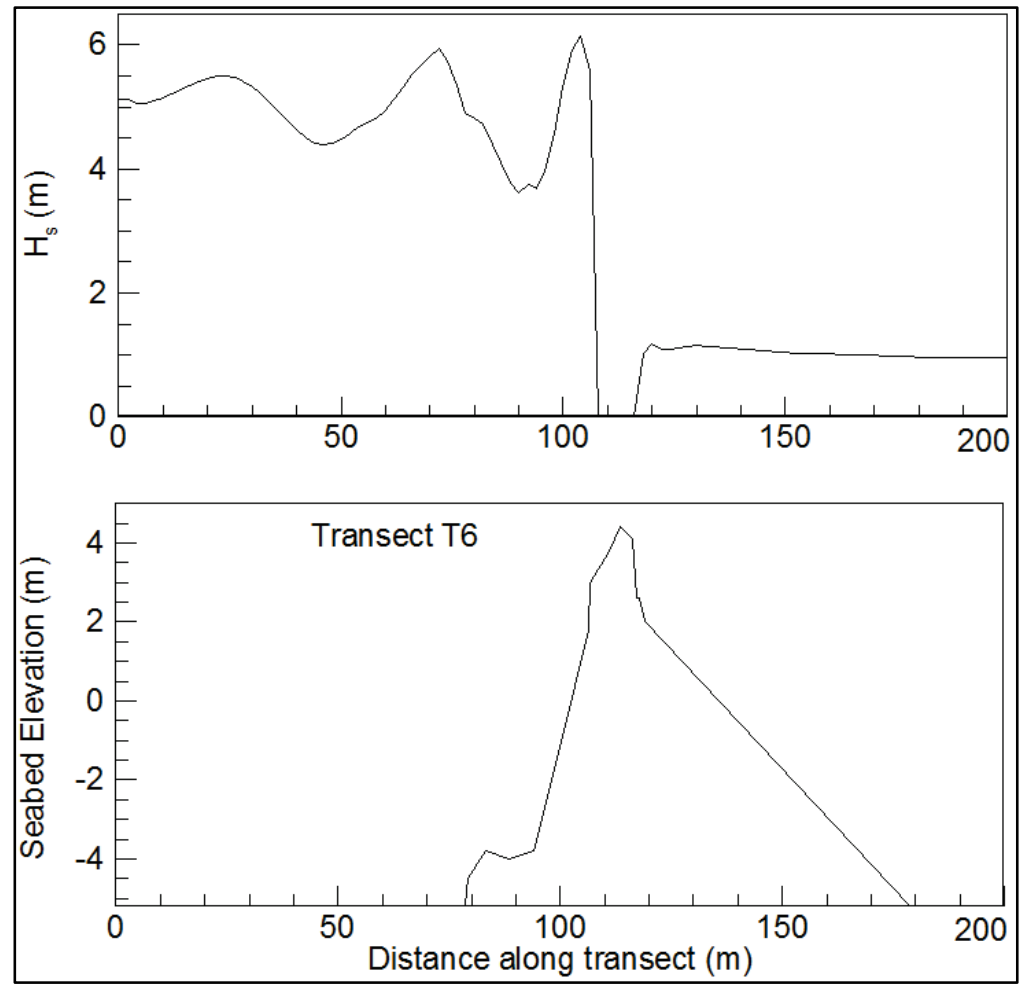

Figure D-11. Overtopping rate and runup height variation along T6 (first 1,000 sec).
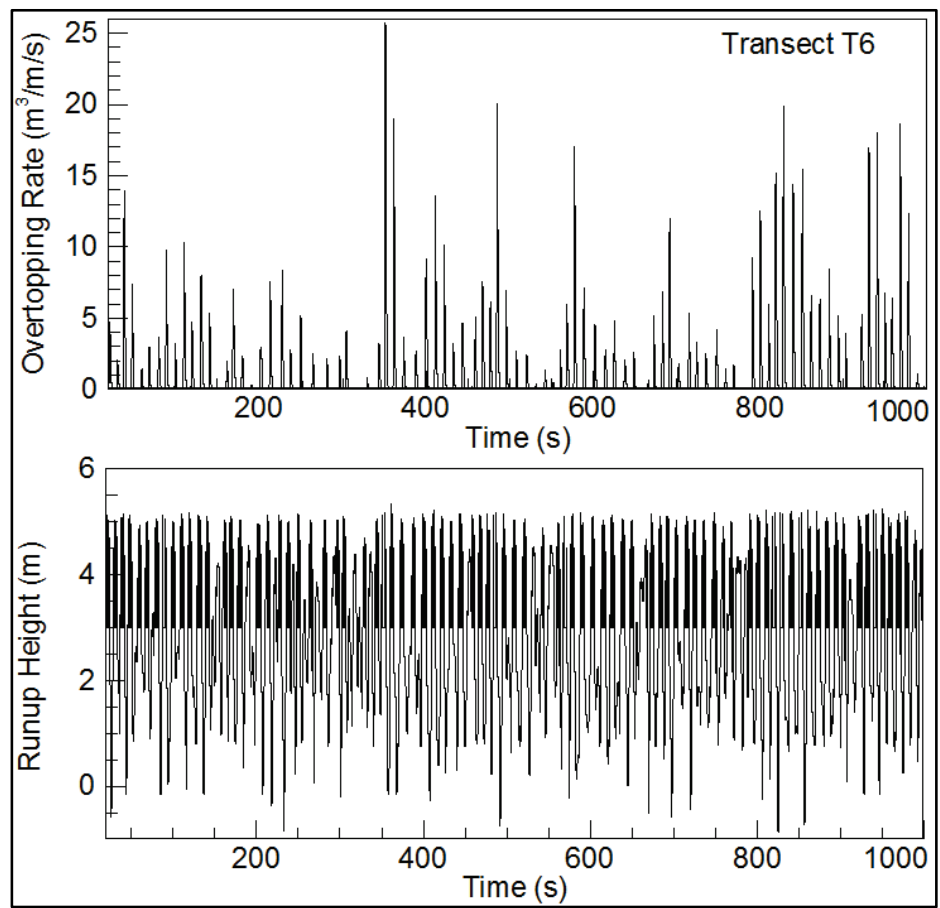
Figure D-12. Time variation of overtopping rate along T6 (entire simulation).

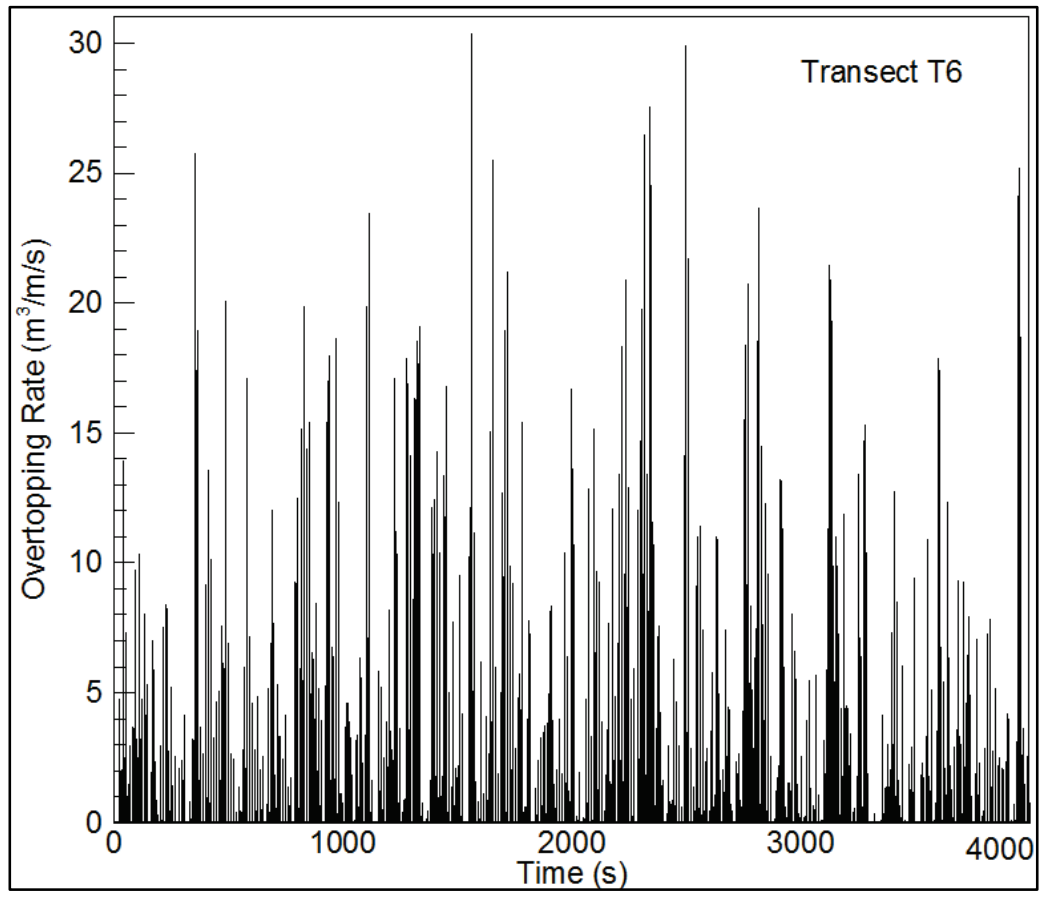

\section{D.5 Results for Transect T7}

Figure D-13. Wave height variation along T7.

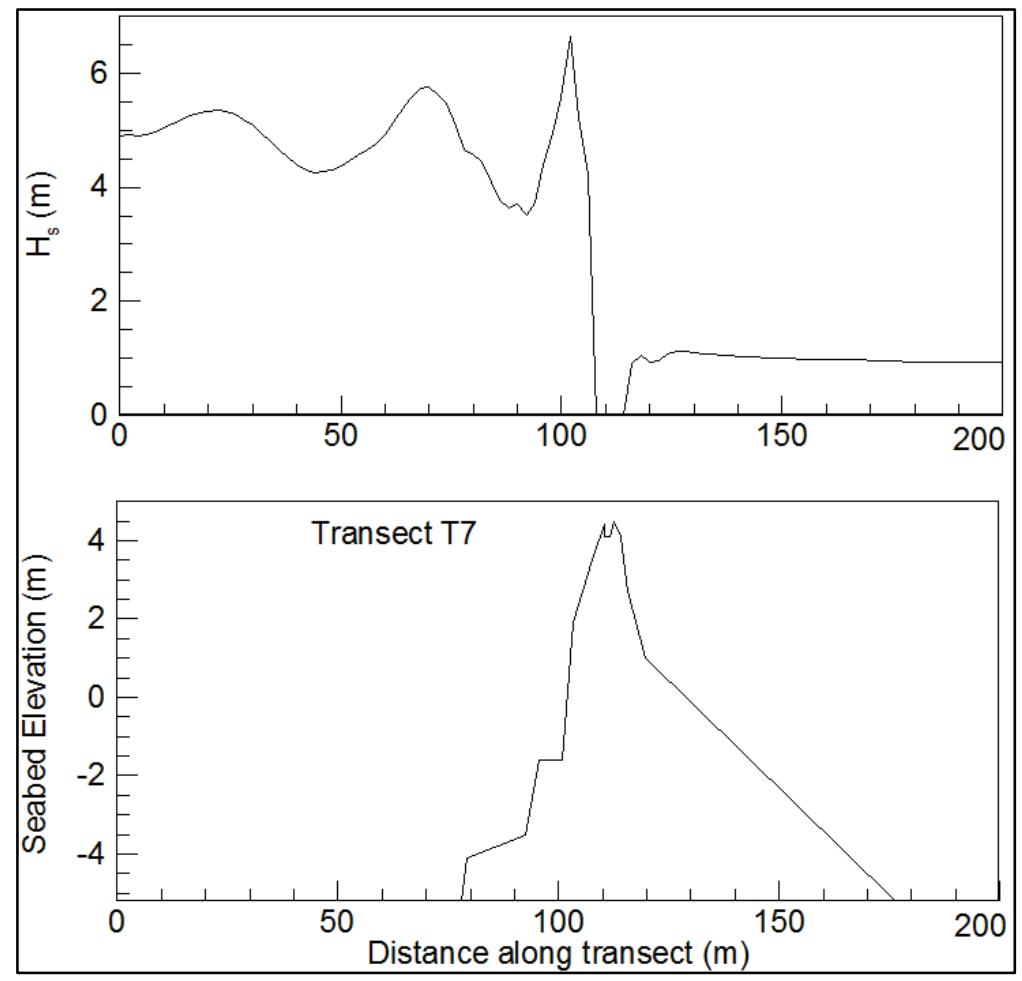


Figure D-14. Overtopping rate and runup height variation along $T 7$ (first 1,000 sec).

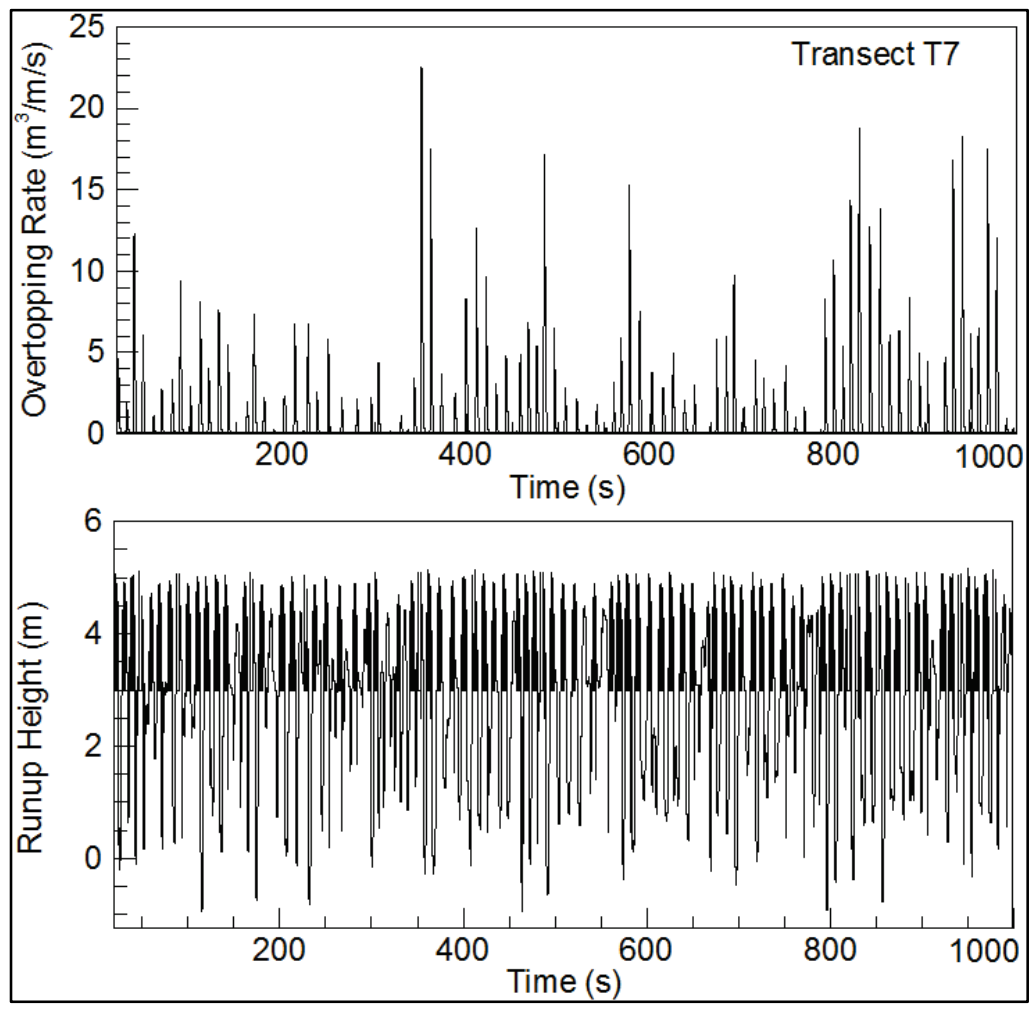

Figure D-15. Time variation of overtopping rate along T7 (entire simulation).

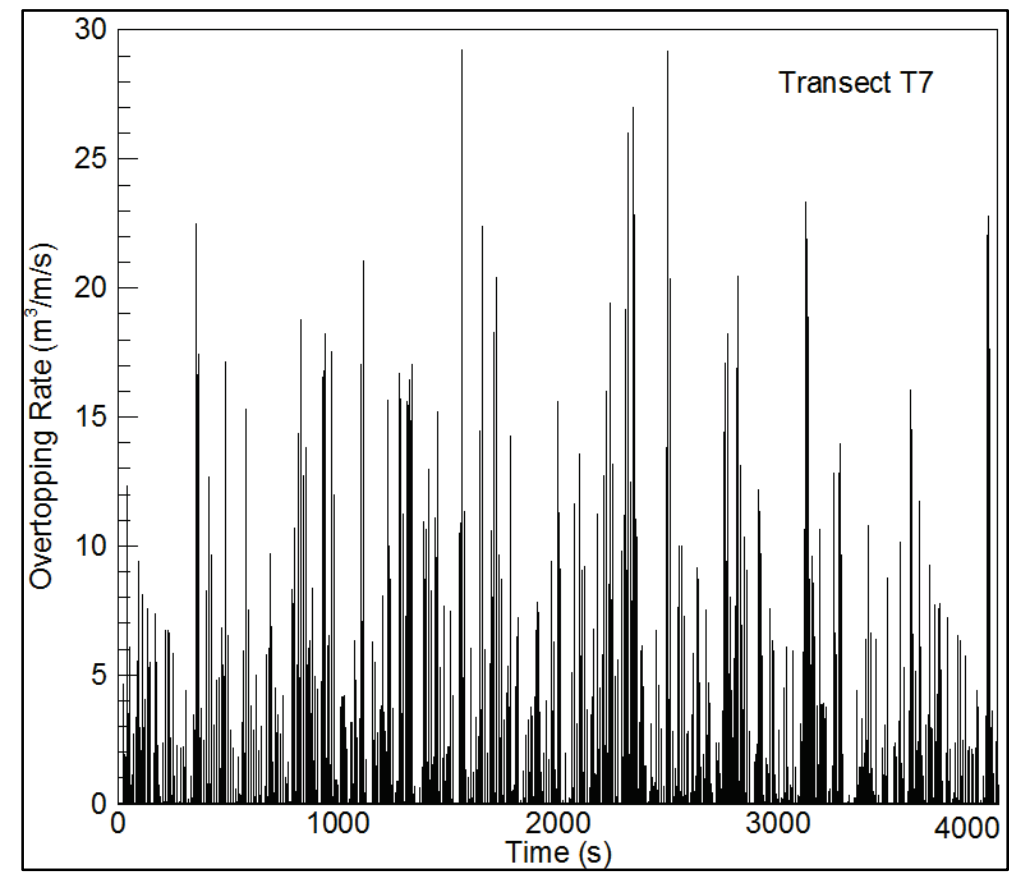




\section{D.6 Results for Transect T8}

Figure D-16. Wave height variation along T8.

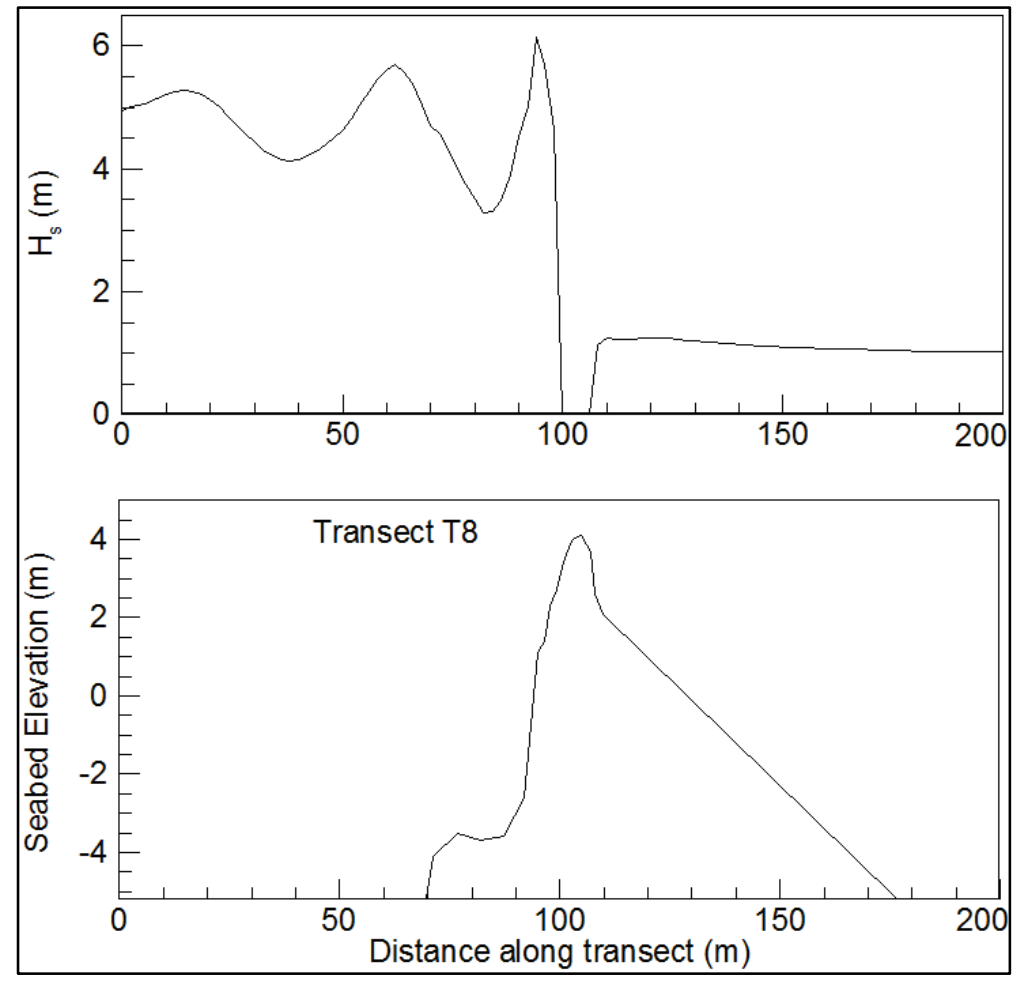

Figure D-17. Overtopping rate and runup height variation along T8 (first 1,000 sec).
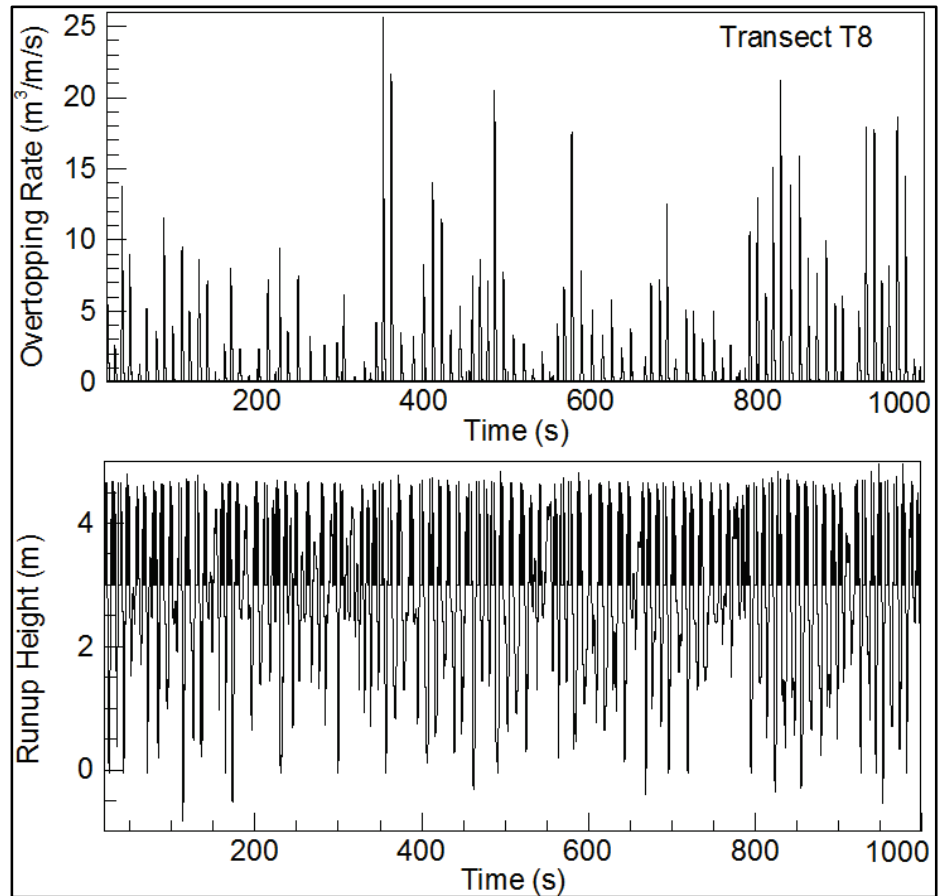
Figure D-18. Time variation of overtopping rate along T8 (entire simulation).

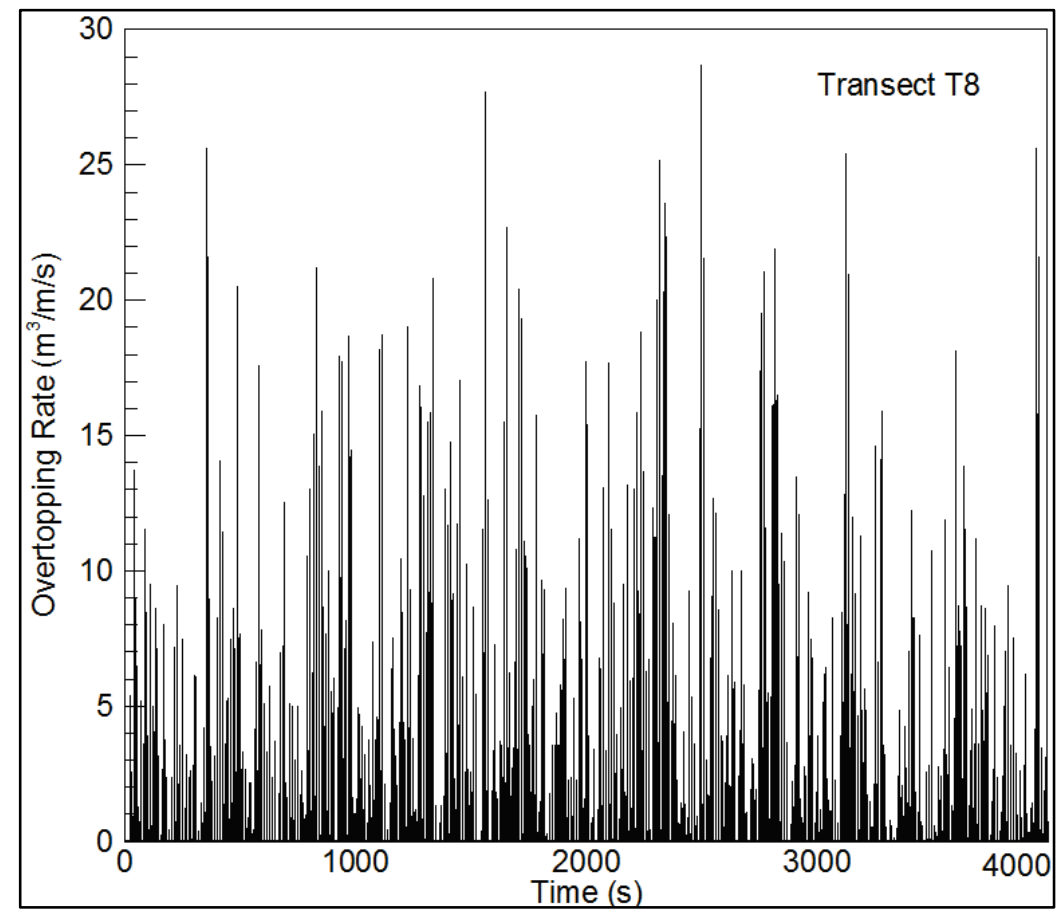

\section{D.7 Results for Transect T10}

Figure D-19. Wave height variation along T10.

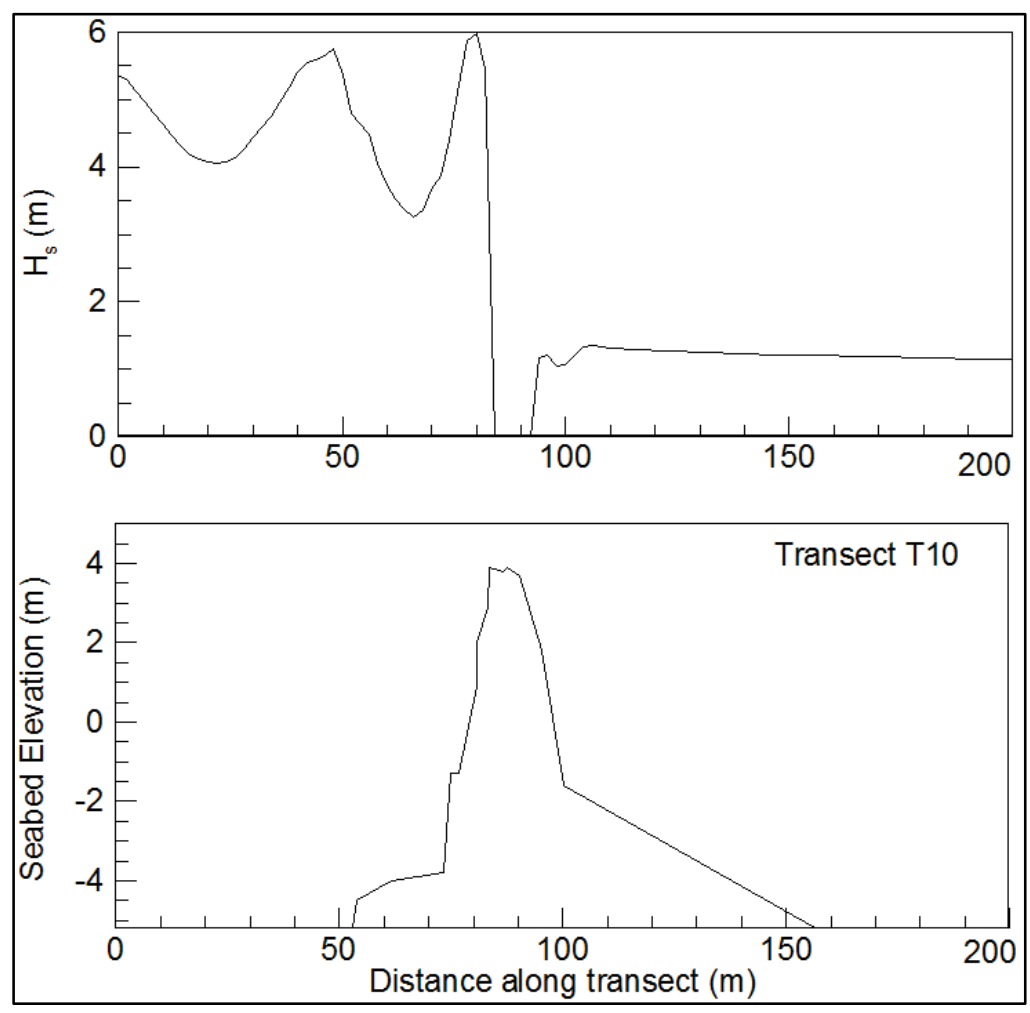


Figure D-20. Overtopping rate and runup height variation along T10 (first 1,000 sec).
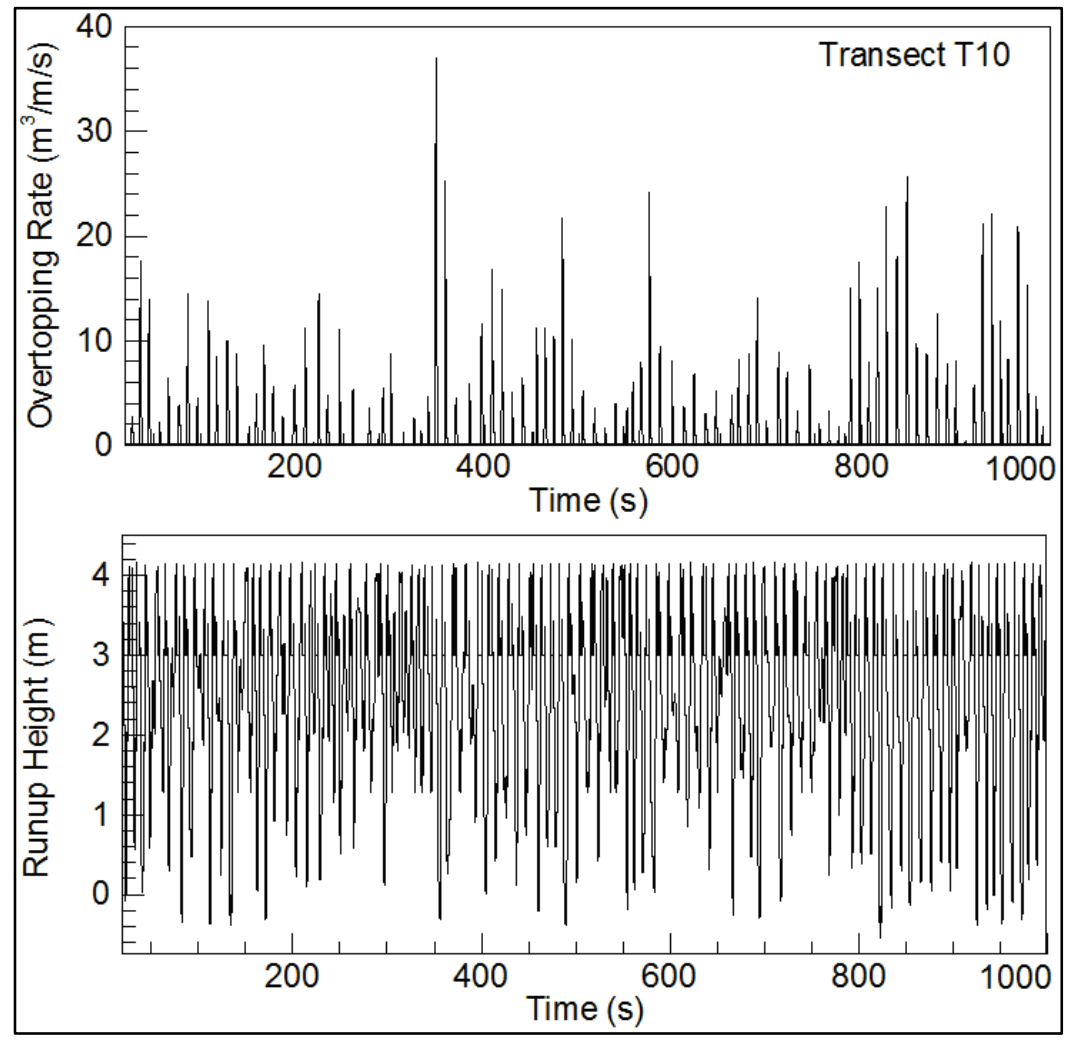

Figure D-21. Time variation of overtopping rate along T10 (entire simulation).

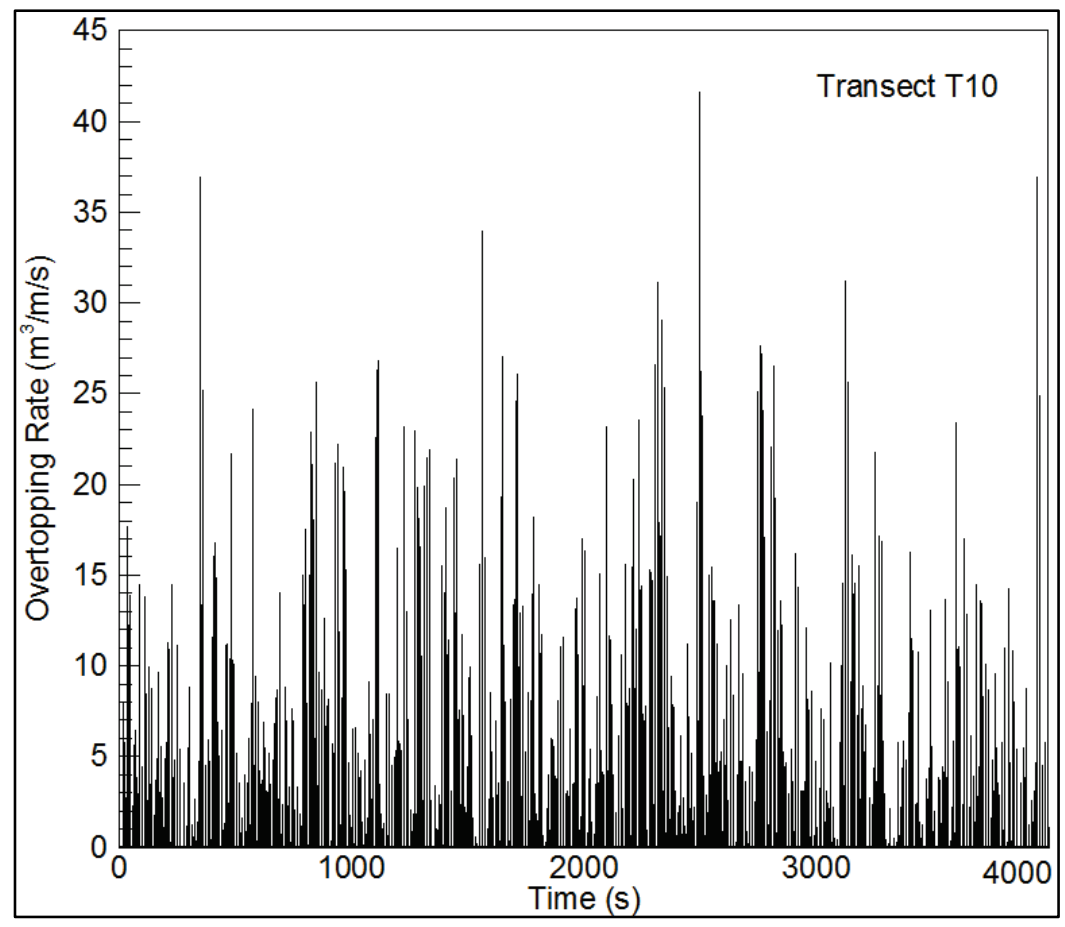




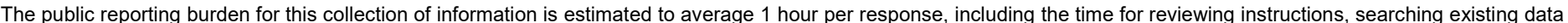

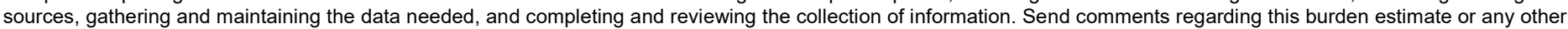
aspect of this collection of information, including suggestions for reducing the burden, to Department of Defense, Washington Headquarters Services, Directorate for Information Operations and Reports (0704-0188), 1215 Jefferson Davis Highway, Suite 1204, Arlington, VA 22202-4302. Respondents should be aware that

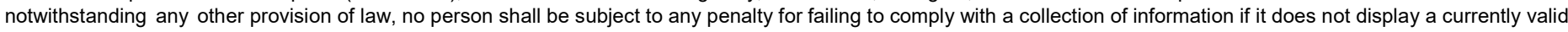
OMB control number.

PLEASE DO NOT RETURN YOUR FORM TO THE ABOVE ADDRESS.

\begin{tabular}{|l|l|l}
\hline 1. REPORT DATE & 2. REPORT TYPE & 3. DATES COVERED (FrOm - To) \\
October 2017 & Final Report
\end{tabular}

\section{TITLE AND SUBTITLE}

Numerical Modeling of Wave Overtopping of Buffalo Harbor Confined Disposal Facility (CDF4)

5a. CONTRACT NUMBER

5b. GRANT NUMBER

5c. PROGRAM ELEMENT NUMBER

5d. PROJECT NUMBER

6. AUTHOR(S)

Zeki Demirbilek, Lihwa Lin, Okey G. Nwogu, Michael C. Mohr, Shanon Chader, and Geoffrey K. Hintz

\section{PERFORMING ORGANIZATION NAME(S) AND ADDRESS(ES)}

Coastal and Hydraulics Laboratory

U.S. Army Engineer Research and Development Center

3909 Halls Ferry Road

Vicksburg, MS 39180-6199

9. SPONSORING/MONITORING AGENCY NAME(S) AND ADDRESS(ES)

Buffalo District, U.S. Army Corps of Engineers

1776 Niagara Street

Buffalo, NY 14207

12. DISTRIBUTION/AVAILABILITY STATEMENT

Approved for public release; distribution is unlimited

\section{SUPPLEMENTARY NOTES}

\section{ABSTRACT}

This report provides details of a numerical modeling study conducted to provide estimates of wave overtopping of the Buffalo Harbor Confined Disposal Facility (CDF4). The CDF4 is situated adjacent to the South Entrance Channel to Buffalo Harbor, NY, along the south shoreline of the Lake Erie. This contaminated material holding facility experiences periodic overtopping during significant storm events. There is concern that overtopping waves may cause the contaminated fine-grain sediments inside the CDF to mobilize, some of which may get transported over the South Entrance Arm Breakwater (SEAB) into the south harbor entrance, navigation channel and harbor complex. The present study focused on predictive estimates of waves and wave overtopping, so flow and sediment transport modeling were not considered. Instead, it was assumed that if flow occurred over the SEAB, some sediment would exit also. Wave processes in the exterior and interior areas of CDF4 were investigated to determine wave runup and overtopping for the existing CDF system using two classes of wave models: CMS-Wave, a spectral wind-wave generation, growth and transformation model, and a nonlinear Boussinesq-type wave model (BOUSS-1D/2D). This report documents details of the modeling study, including data used in the study, results and recommendations.

\section{SUBJECT TERMS}

Buffalo (N.Y.), Contaminated sediments, Dredging spoil, Harbors, Waste disposal sites

\begin{tabular}{|l|l|l|l|l|l|}
\hline \multicolumn{2}{|l|}{ 16. SECURITY CLASSIFICATION OF: } & $\begin{array}{c}\text { 17. LIMITATION OF } \\
\text { ABSTRACT } \\
\text { Unclassified }\end{array}$ & $\begin{array}{c}\text { 18. NUMBER OF } \\
\text { PAGES }\end{array}$ & $\begin{array}{l}\text { 19a. NAME OF RESPONSIBLE PERSON } \\
\text { Zeki Demirbilek }\end{array}$ \\
\cline { 1 - 2 } $\begin{array}{l}\text { a. REPORT } \\
\text { Unclassified }\end{array}$ & $\begin{array}{l}\text { b. ABSTRACT } \\
\text { Unclassified }\end{array}$ & $\begin{array}{l}\text { c. THIS PAGE } \\
\text { Unclassified }\end{array}$ & 180 & $\begin{array}{l}\text { 19b. TELEPHONE NUMBER (Include area code) } \\
601.634 .2834\end{array}$ \\
\hline
\end{tabular}

